\title{
ESTUDO DE PERTURBAÇÕES DO EQUILÍBRIO DE ECOSSISTEMAS AQUÁTICOS MICROSCÓPICOS COMO MÉTODO PARA AVALIAÇÃO DE CONTAMINAÇÃO POR SUBSTÂNCIAS TÓXICAS
}

\section{IZABEL ADELINA RIBEIRO}

Tese apresentada à Faculdade de Saúde Pública da Universidade de São Paulo para a obtenção do título de Doutor em Saúde Pública.

Orientador: Prof. Dr. Samuel Murgel Branco

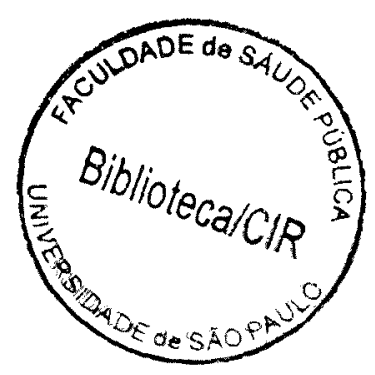

São Paulo

2002 
Autorizo, exlusivamente para fins acadêmicos e científicos, a reprodução total ou parcial desta tese, por processos fotocopiadores.

Assinatura:

Data:

$4 2 + 2 5 \longdiv { 2 0 0 2 }$ die 
Dedico esta tese ao meu pai. 


\section{Agradecimentos}

Agradeço a Fundação de Amparo à Pesquisa do Estado de São Paulo - FAPESP pelo apoio financeiro à realização deste trabalho. Agradecimento adicional, pela aprovação e conseqüente apoio financeiro de estágio em: Técnicas moleculares aplicadas ao estudo de sistemas biológicos, realizado na Universidade de Newcastle - Inglaterra, no presente ano. 
Ribeiro IA. Estudo das perturbações de equilíbrio de ecossistemas aquáticos microscópicos como método para avaliação de contaminação por substâncias tóxicas. São Paulo; 2002 [Tese de Doutorado - Faculdade de Saude Pública da Universidade de São Paulo].

\section{RESUMO}

Sistemas de testes biológicos com espécies representativas têm sido utilizados para avaliar os efeitos adversos causados por substâncias tóxicas no meio aquático. Os bioensaios contam com protocolos definidos porém os organismos estão isolados de seu meio. 0 objetivo deste trabalho é desenvolver um sistema de testes capaz de avaliar os efeitos adversos, não apenas sobre uma única espécie, mas sobre um ecossistema. Para esta finalidade foi construído em laboratório um microecossistema aquático experimental capaz de gerar uma comunidade microbiológica e reproduzir em escala reduzida os componentes de um ecossistema natural formando uma estutura ecológica. Dois grupos de morfológicos foram observados em sequiência ecológica na medida em que as condições do meio eram alteradas. Curvas das dinâmicas de crescimento indicaram comportamento típico para 0 grupo de protozoários ciliados e apresentaram crescimento logarítmico em torno do quarto dia de aeração. Inibição no crescimento foi observado quando concentrações de $12,28 \mathrm{mg}$ de cobre (3,12 ppm) e $2,5 \mathrm{ml} / \mathrm{l}$ de inseticida DDVP 20 são introduzidas nos sistemas. Não foi observada dinâmica típica para o grupo de bactérias filamentosas. Para análise dos resultados foi aplicado teste estatístico de análise de variância. $O$ método não permite o estudo de relações ecológicas que se estabelecem mas fornece informações sobre aspectos teóricos da ecologia referentes ao funcionamento dos ecossistemas e seus atributos como a capacidade de auto-organizar-se. Para maior precisão do método sugere-se estudo da composição da comunidade com a identificação dos microrganismos e métodos modernos de biologia molecular; alongamento do período do experimento e aplicação de modelos populacionais. 
Ribeiro IA. Estudo das perturbações de equilíbrio de ecossistemas aquáticos microscópicos como método para avaliação de contaminação por substâncias tóxicas. São Paulo; 2002 [Tese de Doutorado - Faculdade de Saude Pública da Universidade de São Paulo].

\section{SUMMARY}

Single species toxicity tests are useful in comparing relative toxicities of chemicals in the aquatic environment. Bioassays althouth have the advantages of standardized protocols and objective endpoints the organisms are isolated to their environment. The objective of this study is evaluate the adversed effects caused by chemical compounds not on a single specie but on an aquatic microecossystem community. For that, aquatic microbial microecossystem - small ecosystems held in containers - was built up under laboratory conditions which presented ecological structure. Two differents morfolocial groups of micro-organisms were observed according environmental alterations. Growth curves indicated that the ciliates group has logaritmical development. It was very tipical and happened during the fourth day of experiment. Growth restriction of ciliates was observed at $12,28 \mathrm{mg}$ of cupper (3,12 ppm) and $2,5 \mathrm{ml}$ of DDVP 20 inseticide when were introduced in the systems. The results concerning bacterial group were not conclusive. ANOVA test was applied. This method did not give information that allow studies both ecological patterns and processes but give information about theorical aspects of ecology specially regarding how ecossystems work and their aspects such as auto-organization. In order to develop the method we suggest composition community identification by molecular methods, over time of experiment and mathematical population models can be applied. 


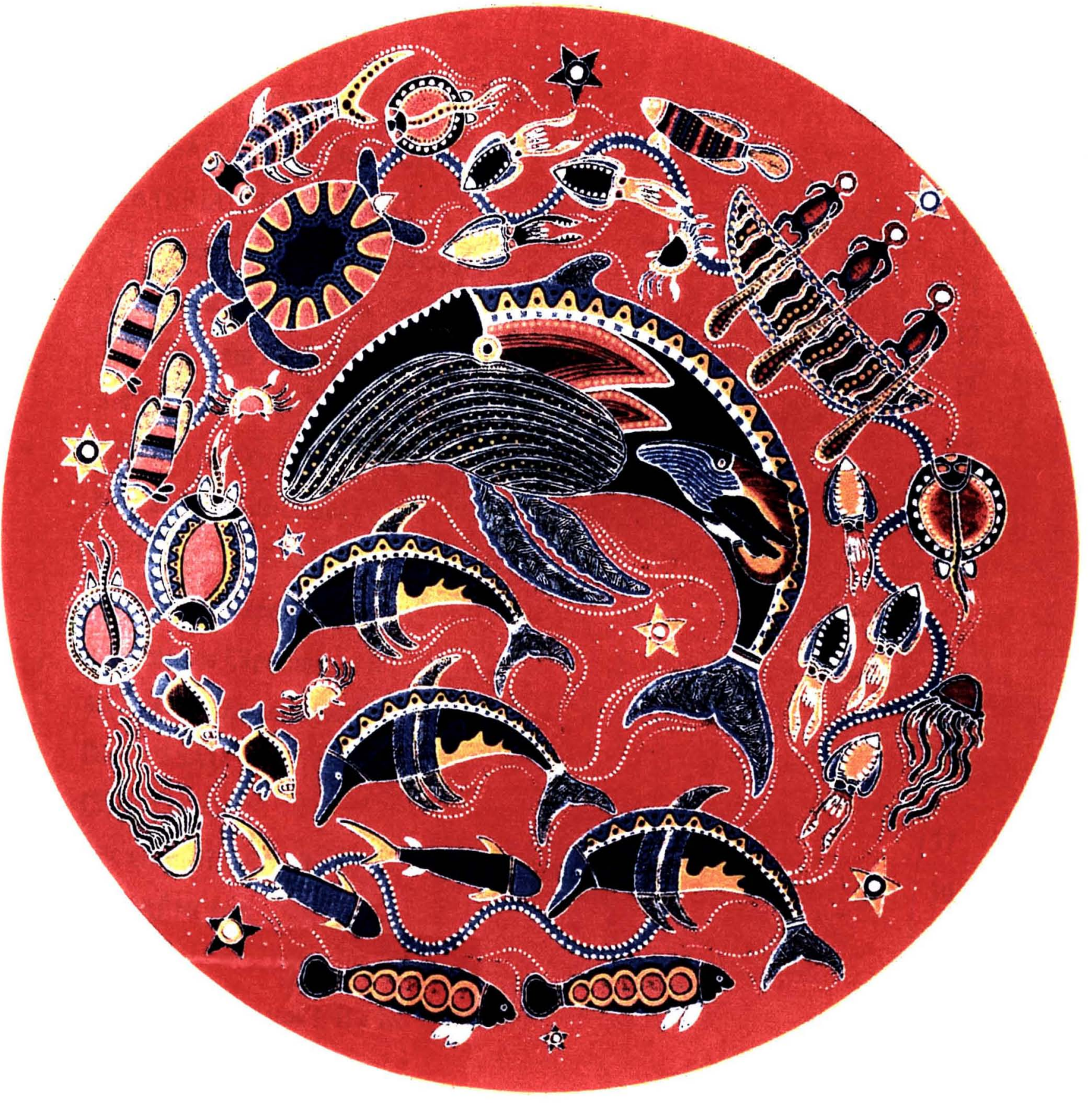


Stiling P. Ecology theories and applications. Cambridge: Cambridge University Press; 1999. 


\section{INDICE}

1 INTRODUÇÃO 01

2 REVISÃO BIBLIOGRÁFICA 04

3 MATERIAL E MÉTODOS 11

3.1 Ecologia dos Microrganismos 11

3.2 Descrição do Modelo 12

3.3 Montagem Experimental 13

3.4 Experimentos 16

4 RESULTADOS $\quad 18$

4.1 Tabulação de Dados 18

4.2 Tratamento Estatístico 19

4.3 Gráficos Experimento I 19

4.4 Grá ficos Experimento II 24

4.5 Gráficos Experimento III 29

5 DISCUSSÃO 34

6 CONCLUSÕES

7 RECOMENDAÇÕES 39

8 REFERÊNCIAS $\quad 40$

ANEXO 1 Al

Tabelas Experimentos I; II e III

$\begin{array}{ll}\text { ANEXO } 2 & \text { A29 }\end{array}$

Análise de Variância - Experimentos I; II e III

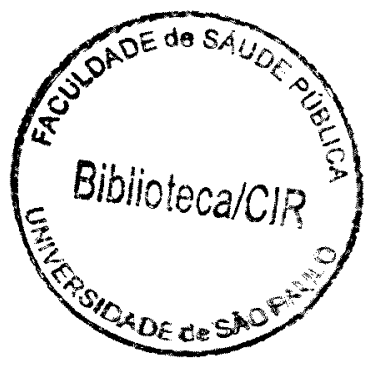




\section{INTRODUÇÃO}

Muitos têm sido os esforços para o desenvolvimento de testes de toxicidade capazes de prever e avaliar os efeitos ecológicos e sanitários provocados por uma imensa variedade de resíduos químicos e orgânicos, potencialmente tóxicos, provenientes de descartes industriais e agrícolas que são lançados direta ou indiretamente no meio aquático.

Duas maneiras são atualmente empregadas para a avaliação e controle no lançamento despejos. A primeira através de limites numéricos de substâncias especificas e a segunda, por meio de testes biológicos de toxicidade ou ensaios biológicos. Os bioensaios avaliariam a potência relativa de uma substância quimica através da comparação de seus efeitos sobre um organismo vivo com uma preparação padrão (RAND e PETROCELLI 1985).

Muito embora, os bioensaios possam estimar com maior segurança o impacto de um dado efluente no meio aquático, há dificuldades que restringem a sua utilização. Em geral o cultivo de animais - em geral aquáticos, constitui a primeira dificuldade. A restrição principal, é a de que os organismos encontram-se isolados de seu meio. No ambiente natural, os organismos estão inseridos no contexto dinâmico das condições naturais.

A principal limitação quanto ao controle por meio de padrões numéricos de emissão, é a de que, mesmo idenficando-se todas as substâncias potencialmente tóxicas, permanece a impossibilidade de se determinar a susceptibilidade biológica aos agentes tóxicos que compõem o efluente. Ou seja, o efeito final de uma solução contendo várias substâncias tóxicas não consiste simplesmente, na somatória dos efeitos tóxicos parciais. 
Desse modo. sistemas de teste ou modelos que consistam em tecnologia de fácil utilização, capazes de avaliar o mais realísticamente possivel os efeitos danosos, provocados pelos tóxicos, no ambiente aquático, têm sido objeto de pesquisa em nível internacional.

Quanto aos efeitos danos de uma determinada mistura, vale citar o desenvolvimento do teste de raízes de cebola como índice de indicação preliminar da presença de substâncias tóxicas ou inibidores biológicos no meio aquático. De baixo custo e de fácil utilização, o teste, originalmente desenvolvido por FISKEJÖ (1993), foi padronizado para as condições brasileiras por RIBEIRO (1997). Atualmente o teste é objeto de trabalhos de pesquisa em laboratórios de universidades no Brasil com sucesso nas repetições de molde.

Já o desenvolvimento de bioensaio com a utilização de espécies de maior sensibilidade e representativas de seu meio, é apresentado por SÁFADI (2001). Em RODRIGUES (2001) a avaliação de contaminação de pesticidas é feita com a utilização de mú ltiplas espécies.

O presente trabalho tem por objetivo o desenvolvimento de um sistema de testes capaz de avaliar os efeitos da contaminação de substâncias tóxicas no meio aquático com um desafio adicional. A avaliação dos efeitos induzidos de um tóxico não seria baseada na exposição de espécies únicas, mas sobre uma inteira comunidade, uma miriade de espécies que comporiam e formariam uma estrutura ecológica definida.

Neste caso, a investigação dos efeitos dos poluentes no meio aquático é conduzida através da abordagem da organização sistêmica. Ou seja, os parâmetros de avaliação são ecossistêmicos. Sendo que a quantificação dos efeitos induzidos pelo tóxico é dada pelas mudanças ou efeitos na estrutura do sistema ecológico, formado experimentalmente, em laboratório (CAIRNS e PRATT 1989). 
Para identificar os efeitos na estrutura ecológica do ecossistema experimental, isto é sobre os componentes deste ecossistemas, os organismos de diferentes grupos taxonômicos são identificados e quantificados em um momento específico. Análises comparativas na ausência e presença do tóxico são realizadas.

Pode-se dizer que o presente trabalho faz fronteira com as modernas áreas da ecologia tanto terrestre quanto aquática, as quais têm se utilizado de sistemas ecológicos experimentais para facilitar o estudo e a compreensão dos níveis de organização e das relações existentes entre os vários elementos que compõem os ecossistemas naturais.

Os sistemas ecológicos experimentais também denominados de microecossistemas ou microcosmos, simulariam, em escala laboratorial, o funcionamento dos ecossistemas naturais. A maior vantagem de utilização e desenvolvimento é a de que nestes modelos, a complexidade e outros atributos únicos como a capacidade de auto-organização e automanutenção, inerentes aos ecossistemas naturais, bem como seus componentes, estão presentes.

No presente trabalho, muito embora, o estudo dos efeitos tóxicos sobre a estrutura do ecossistema formado objetive aplicação prática, conceitos acerca dos níveis de organização e processos ecológicos são necessários e inerentes à própria concepção do modelo. Desse modo, no decorrer dos capítulos são apresentados aspectos teóricos de maior relevância pertinentes à este campo de aplicação da ecologia, atualmente denominada de ecotoxicologia. 


\section{REVISÃ O BIBLIOGRÁ FICA}

Foi no início de 1950 que muitos ecologistas começaram a simplificar os estudos dos ecossistemas simulando-os em montagens experimentais em laboratório, de forma a estarem contidos em diferentes recipientes como frascos e aquários, os quais foram posteriormente chamados de ecossistemas artificiais (CAIRNS e NIEDERLEHNER 1995).

METCALF (1971) apresenta uma destas montagens com a denominação de ecossistema modelo e contendo espécies representativas de vários níveis tróficos formando cadeia alimentar com produtores primários, consumidores e microrganismos decompositores. Desse modo, foram quantificados com elementos autótrofos e heterótrofos, os processos ecológicos como produção fotossintética, respiração e ciclo de nutruientes.

Mais a frente o termo ecossistema artificial passa a ser referenciado como microcosmo implicitando a idéia de pequena réplica do todo. Em CAIRNS e NIEDERLEHNER (1995) microcosmos são modelos em escala reduzida de ecossistemas naturais ou porções de sistemas naturais mantidos em recipientes artificiais.

Em WIMPENNY (1988) os microcosmos são montagens laboratoriais de sistema natural e tendem a ser compactos. Podem ser sistemas fechados ou parcialmente fechados para troca de espécies e materiais. Apresentam geralmente, grande número de espécie microscópicas. 
Em BEYERS e ODUM (1993) microcosmos ou microecossistemas (BEYERS 1963) são ecossistemas em menor tamanho e mantidos em recipientes. Para ODUM (1983) são pequenos mundos auto-suficientes capazes de simular em menor escala os ecossistemas naturais com todos os seus componentes.

Em princípio, o objetivo de desenvolver modelos, em escala laboratorial, de sistemas ecológicos naturais era o de facilitar a compreensão das inter-relações existentes entre os vários elementos que compõem os ecossistemas e o modo como estes operam. $\mathrm{O}$ que proporcionou grande avanço nos aspectos teóricos da ecologia.

Além do campo da ecologia teórica, os microecossistemas, principalmente os de tipo estritamente aquáticos começaram a ser aplicados no estudo das vias pelas quais os ecossistemas aquáticos naturais são afetados e respondem às mais variadas perturbações provocadas pela introdução de substâncias químicas sintéticas.

Por outro lado, com objetivos afins, sistemas de testes de toxicidade para avaliação dos efeitos de resíduos descartados de operações industriais com a utilização de organismos aquáticos têm sido realizados deste 1940 (CAIRNS e NIEDERLEHNER 1995).

$\mathrm{O}$ rápido desenvolvimento de testes de toxicidade com a utilização de organismos representativos, deu origem à toxicologia aquática, definada por RAND e PETROCELLI (1985) como o estudo qualitativo e quantitativo dos efeitos tóxicos ou adversos de substâncias químicas e outros materiais antropogênicos ou xenobióticos sobre os organismos aquáticos. Englobando ainda, o estudo do transporte, distribuição e destino final dessas substâncias no meio aquático. 
Mais recentemente, a aplicação de microcosmos ou microecossistemas na avaliação dos efeitos nas propriedades e estruturas de comunidades inteiras ou sistemas ecológicos estreitou a fronteira entre a ecologia e a toxicologia aquática o que deu origem à ecotoxicologia. Esta nova ciência é definida por CAIRNS e NIEDERLEHNER (1995) como sendo o estudo dos efeitos tóxicos nos constituintes dos ecossistemas.

Se por um lado, os testes de toxicidade apresentam inúmeras vantagens como, por exemplo contar com protocolos bem definidos, que proporcionam o estudo quantitativo dos efeitos tóxicos nos organismos, os microecossistemas apresentariam a vantagem de prover respostas de muitas espécies simultaneamente, incluindo os efeitos dos tóxicos sobre as interações das espécies e destas com o meio.

Muito embora, os microecossistemas pareçam constituir modelos mais realísticos de estudo das interferências provocadas pelas substâncias tóxicas no meio aquático, a aplicação direta dos resultados está longe de ser tarefa simples.

A comunidade, o fluxo de energia - como num sitema aberto - e a ciclagem de materiais são os componentes básicos de um ecossistema. O fluxo de energia que entra no sistema produziria estruturas bióticas e ciclagem de materiais entre as parte vivas e não vivas (ODUM 1983). Comunidade, população e organismo, interagiriam regularmente para formar um todo unificado.

A consequiência importante de que o ecossistema funcionaria segundo padrão hierárquico é que, à medida que os componentes combinam-se para produzir sistemas funcionais maiores, distintas propriedades emergem (SALT 1979). Não pelo fato de que a natureza básica dos componentes seja alterada, mas devido à interação propriamente dita dos componentes (ODUM 1983). 
O grau em que os ecossistemas realmente operam como sistemas auto-organizadores, à maneira dos organismos que os compõem, ainda é assunto de debate (WILSON e BOTKIN 1990). Contudo, a extenção dos resultados obtidos nos microecossistemas aquáticos aos ecossistemas naturais apresentaria o mesmo problema que comparar dois ecossistemas naturais entre eles.

Vale ressaltar, que cada ecossistema natural possui estruturas próprias de modo que, quando uma mesma espécie ocorre em dois ecossistemas, encontram-se inseridas em contextos distintos e apresentam relações de sobrevivência diferentes (BOUDOU e RIBEYRE 1988).

O mesmo ocorre na comparação entre microecossistemas experimentais em que, comparações qualitativas dos efeitos provocados na estrutura ou comunidades dos microecossistemas, são mais adequadas que comparações quantitativas (BOUDOU e RIBEYRE 1988), muito embora apresentem determinado grau de similaridade.

Muito embora a dificuldade de estudo quantitativo dos efeitos que as substâncias tóxicas provocam nos ecossistemas aquáticos naturais, em conseqüência de capacidade de auto-organização, conforme foi salientado na bibliografia, possam também ser observáveis em modelos de sistemas ecológicos em menor escala, os modelos experimentais objetivam a definição de protocolos.

A padronização de modelos facilitaria o estudo comparativo dos resultados com outros sistemas de testes. Várias metodologias fazem referência à composição da comunidade do microecossistema experimental e compreendem desde uma comunidade totalmente composta por organismos cultivados em laboratório, até espécies coletadas em ambiente natural. 
Estudo para a padronização de microecossistema aquático com espécies representativas de vários níveis tróficos, é apresentado por BOUDOU e RIBEYRE (1988). O estudo resultou em um modelo capaz de comprovar a sensibilidade a uma variedade de compostos químicos - metais pesados, antibióticos e inseticidas -, nas relações ecológicas existentes na comunidade do microecossistema. Os resultados são comparáveis a outros sistemas de testes e o protocolo metodológico é apresentado.

Microecossistemas experimentais foram usados para avaliar os efeitos do cobre, atrazine e muitos outros compostos nos organismos desse microcosmo. Em geral esses organismos têm papel na biodegração, biotransformação e bioacumulação destes contaminantes (TAUB 1976).

Metodologias com colonização espontânea de microrganismos também são apresentadas na bibliografia especializada. Comunidades de protozoários constituindo um microecossistema, foram usadas para estimar concentrações permissíveis de cobre em ecossistemas aquáticos. 0 substrato foi mantido a meio metro do nível da água de rio por 14 dias e a comunidade foi composta por colonização natural (PRATT, NIEDERLEHNER, BOWERS e CAIRNS 1987).

A riqueza taxonômica da comunidade foi afetada em concentrações maiores que 12,7 $\mathrm{mg} / \mathrm{l}$ após 21 dias de exposição. Redução de populações individualmente ocorreu em 8,7 $\mathrm{mg} / \mathrm{l}$. Níveis associados a danos de ecossistemas naturais maiores que $23,0 \mathrm{mg} / \mathrm{l}$ (PRATT, NIEDERLEHNER, BOWERS e CAIRNS 1987). 
Para CALOW (1995), a demonstração de efeitos ecológicos sobre diversos níveis tróficos requer que mais de uma espécie esteja presente em cada nível tró fico e que possam ser demonstradas mudanças de espécies dominantes ou diversidade de espécies. Poluentes muitas vezes eliminam espécies sensíveis e aumentam a abundância de outras.

$\mathrm{O}$ aumento da complexidade de um determinado modelo microecossistêmico conduz a aumento analítico dos problemas. É provável que as mudanças estruturais sejam mais fácilmente verificáveis, em sistemas que envolvam organismos de níveis tróficos mais baixos (MAYFIELD 1995).

Microecossistemas experimentais constituídos exclusivamente por microrganismos têm sido amplamente desenvolvidos. Nos últimos cinco anos, técnicas de biologia molecular têm sido utilizadas para facilitar o estudo da diversidade e da dinâmica das comunidades microbiológicas. Dentre estas, a técnica de Gradiente de Desnaturação em Gel Eletroforese DGGE (MUIZER e SMALLA 1998).

Para MUIZER (1999), a classificação dos microrganismos por traços morfológicos é insuficiente para explorar o enorme reservatório da diversidade biológica. As técnicas atuais de biologia molecular permitem que os microrganismos sejam agrupados de acordo com seu perfil genético e possibilitam a identificação de microrganismos que não poderiam ser indentificados por métodos microscópicos (CURTIS e CRAINE 1998).

Aliado às técnicas moleculares, modelos teóricos têm sido utilizados para explicar as relações de diversidade na dinâmica populacional. É o caso da teoria da ilha biogeográfica (MCARTHUR e WILSON 1967 ) aplicada à microecossistemas (CURTIS e HEAD 1999 ). Ou ainda, o modelo de competição por fonte única de alimento, formulado originalmente 
para verificar se a competição pela fonte impactuaria na diversidade biológica do sistema resource ratio theory-.

Indices numéricos derivados de medidas quantitativas diretas também podem ser aplicados no estudo da estrutura da comunidade do microecossistema. Dentre os quais, o índice de abundância relativa - número total de indivíduos de uma espécie pelo total de indivíduos da comunidade -, a riqueza taxonômica - número de unidades taxonômicas ou espécies - a igualdade - número de indivíduos distribuídos entre as espécies - assim como os indices de diversidade.

Como predizer o estado futuro de um ecossistema e os efeitos provocados no seu equilíbio dinâmico é outra questão da ecologia teórica e se refere à relação entre complexidade e estabilidade.

Por muitos anos acreditou-se que a complexidade, principalmente a relacionada à diversidade de espécies, aumentaria a estabilidade ecológica (HUTCHINSON 1959), MAY (1975) demonstrou que para modelos populacionais de tipo Lotka-Volterra, a estabilidade é estabelecida em função da complexidade da cadeira alimentar.

Para BOTKIN e WILSON (1990) a conexão, é ainda desconhecida e seria desejável desenvolver modelos para testar a importância da complexidade, sob vários aspectos, na estabilidade dos ecossistemas. 


\section{MATERIAL E MÉ TODOS}

\subsection{Ecologia dos Microrganismos}

Microrganismos são importantes membros de ecossistemas, especialmente por operarem na base da cadeia alimentar na conversão de detritos provenientes de fontes externas transformando-os em biomassa. Quando bem escolhidos serviriam como chave de representatividade de organismos superiores presentes nos ecossistemas naturais (CAIRNS e PRATT 1989).

Microecossistemas microbiológicos são desenvolvidos com a suposição de que os organismos teste sejam representativos de todo o corpo de organismos que compreendem o meio ambiente (CAIRNS e PRATT 1989). Ou ainda, para KELLY e HARWELL (1989) microrganismos pertencentes à niveis tróficos mais baixos serviriam como alerta ou indicadores potenciais de danos a um ecossistema aquático.

Desse modo, o objetivo máximo deste trabalho é o desenvolvimento de um sistema de teste partindo da hipótese de que possa ser utilizado como método de avaliação na indicação preliminar de contaminação de ecossistema aquático natural por substâncias tóxicas.

Para essa finalidade um microecossistema aquático experimental, que seja capaz de gerar uma comunidade biótica aquática e microscópica, é construído em escala laboratorial. Um meio ambiente, formado a partir das condições impostas pelo próprio desenho experimental, reuniria condições ambientais favoráveis para a formação de uma estrutura ecológica representada por uma comunidade de microrganismos, que the seria típica, observável por métodos microscópicos. 
Após a caracterização da estrutura e da dinâmica da comunidade do microecossistema formado seria possível estudar as alterações ecológicas provocadas pela introdução de substâncias tóxicas ao sistema.

\subsection{Descrição do Modelo}

A comunidade microbiológica formada consiste exclusivamente de microrganismos aeróbios. O substrato utilizado é mantido sob aeração contínua com a finalidade de constituir um meio ambiente favorável à geração destes microrganismos.

O regime de funcionamento do sistema não prevê realimentação do substrato. Desse modo, as condições ambientais devem modificar-se em função do tempo e da presença dos microrganismos gerados. As condições iniciais do meio ambiente favoreceriam a geração de microrganismos aptos a consumir alimento em forma dissolvida.

$\mathrm{Na}$ medida em que o alimento em forma diluída for se escasseando pela ação dos primeiros microrganismos gerados, a nova condição do meio favoreceria o surgimento de diferentes formas com mecanismos nutricionais diferentes numa seqüência ecológica característica.

Não há realimentação de substrato, portanto a fonte de energia do sistema é única e pontual simplificando o método. $\mathrm{O}$ modelo por ser fechado para troca de materiais, favorece sua aplicabilidade prática, contudo vale ressaltar que em sua dinâmica não corresponde à dinâmica existente em um ecossistema natural. 
O modelo seria, pois, indicado somente para avaliação dos efeitos dos contaminantes na comunidade microscópica em um determinado momento, o que corresponderia ao efeito de um determinado efluente imediatamente após o seu lançamento no meio aquático.

Durante os ensaios preliminares, foi estudado o desenvolvimento de um modelo experimental em equilibrio dinâmico de um sistema aberto, com entrada e saída de nutrientes. Porém, o seu prosseguimento não foi possível, por dificuldades de operação. Por exemplo, o entupimento do sistema de mangueiras de alimentação por coagulação do substrato, o que impediu que o fluxo de alimentação ocorresse de forma uniforme e contínua.

\subsection{Montagem Experimental}

O meio aquático foi colocado em cubas de vidro com capacidade para dois litros e mantido sob aeração contínua. A aeração dá-se por meio de compressores e difusores de aquário. 0 ar chega ao sistema através de mangueiras de silicone de 5 milímetros de diâmetro aproximadamente. A difusão do ar é feita por apenas um difusor e direcionada de baixo para cima de modo a favorecer a homogeinização do meio.

Estes recipientes foram parcialmente tampados, mantidos e em temperatura ambiente e distantes da incidência de luz solar. 
A) Composição do substrato:

Substrato orgânico diluído constituído por leite longa vida desnatado com capacidade redutora em termos de demanda bioquímica de oxigênio (DBO) = $35.449 \mathrm{mg} / \mathrm{l}$.

B) Medidas de diluição do substrato:

O substrato foi diluído em água destilada em proporções apropriadas à uma DBO de $350 \mathrm{mg} / \mathrm{l}$.

\section{Tabela 1 - Composição do substrato}

\begin{tabular}{|c|c|}
\hline \multicolumn{2}{|c|}{$\begin{array}{c}\text { leite longa vida desnatado Parmalat } \\
\text { Composição em 100 ml }\end{array}$} \\
\hline Nutrientes & Quantidade \\
\hline Proteínas & $3,18 \mathrm{~g}$ \\
\hline Glicídios & $4,90 \mathrm{~g}$ \\
\hline Lipídios & $0,50 \mathrm{~g}$ \\
\hline Fibras alimentares & $0,10 \mathrm{~g}$ \\
\hline Cálcio & $141 \mathrm{mg}$ \\
\hline Fósforo & $94 \mathrm{mg}$ \\
\hline Potássio & $115 \mathrm{mg}$ \\
\hline Sódio & $50 \mathrm{mg}$ \\
\hline
\end{tabular}




\section{C) Replicabilidade:}

- 5 sistemas controle;

- 5 sistemas para cada concentração de substância tóxica aplicada.

C) Tempo de duração do experimento:

- Nove dias consecutivos.

E) Amostragem:

Em cada sistema foram analisadas total de 12 amostras, a cada 24 horas, durante os 9 dias consecutivos. As amostras eram retiradas com pipeta de vidro de modo aleatório e a cada 24 horas.

F) Observação - classificação e contagem dos microrganismos:

A observação da dinâmica, contagem e identificação dos microrganismos foi feita por análise microscópica direta em aumento de 40 vezes. A amostra era colocada numa câmara de contagem de células - tipo Sedwick Rafter - com capacidade para $1 \mathrm{ml}$ de amostra. 
G) Medidas de pH - oxigênio dissolvido e temperatura da água:

Vale ressaltar que não faz parte do presente trabalho a determinação de variáveis a serem controladas com vistas à uma padronização. Desta forma as medidas de $\mathrm{pH}$, temperatura da água e oxigênio dissolvido foram executadas como um monitoramento das condições resultantes no meio.

\subsection{Experimentos}

A) Experimentos I e II - com aplicação de sulfato de cobre;

B) Experimento III - com aplicação de inseticida - DDVP-20.

Vale ressaltar que por tratar-se de fase de ensaios preliminares o experimento I foi realizado sem sistemas controle. Dessa forma, para análise foram utilizados os resultados dos sistemas controle do experimento II.

A escolha do sulfato de cobre e do DDVP-20 foi baseada no uso intensivo que deles é feito em diversos cultivos agrícolas, produzindo resíduos que freqüentemente são arrastados pelas chuvas, tendo acesso à coleções de àgua. Além disso, o cobre é amplamente utilizado em processos industriais e no controle da qualidade de água para fins de abastecimento, é amplamente utilizado em outros sistemas de testes, além de integrar a lista de metais pesados com limites permissíveis estipulados pela legislação ambiental. 
Concentrações aplicadas:

$\underline{\text { Sulfato de cobre }-\mathrm{CuSO}_{4}} \underline{.5 \mathrm{H}_{2}} \underline{\mathrm{O}}$
1) controle;
2) $\quad 0,78 \mathrm{ppm}$ de cobre $=3,07 \mathrm{mg} / \mathrm{l}$;
3) $1,56 \mathrm{ppm}$ de cobre $=6,14 \mathrm{mg} / \mathrm{l}$;
4) $\quad 3,12 \mathrm{ppm}$ de cobre $/ \mathrm{l}=12,28 \mathrm{mg} / \mathrm{l}$.

Inseticidade e acaricida DDVP-20: fosfato de 0,0-dimetil-2,2-diclorovinil (Dichlorvos). Organofosforado - classe toxicológica II :

De uso comum no controle de pragas que atacam desde hortaliças até culturas de algodão e cítrus ( ANDREI 1985).
1) controle;
2) $0,62 \mathrm{ml} /$;
3) $1,25 \mathrm{ml} / 1$;
5) $2,5 \mathrm{ml} / \mathrm{l}$. 


\section{RESULTADOS}

A análise das amostras demonstrou que à partir das primeiras 24 horas de aeração ocorreu a geração de bactérias filamentosas. A segunda geração de microrganismos é de a protozoários do tipo ciliados livres e ocorre entre o terceiro e quarto dia de aeração. As curvas de crescimento do primeiro e segundo grupos são bastante distintas. $\mathrm{O}$ crescimento de ciliados é notório, apresentando fase de progressão logarítmica e atingindo sua concentração máxima por volta do quarto dia de experimento.

A aplicação das substâncias tóxicas no momento de progressão logarítmica facilitaria a análise das relações numéricas e quantificação dos efeitos provocados. $\mathrm{O}$ efeito direto analisado seria a inibição do crescimento dos ciliados livres e outras possíveis modificações na estrutura da comunidade do microecossistema.

A identificação microscópica foi feita conforme BRANCO (1986) e BERK e GUNDERSON (1993).

\subsection{Tabulação dos Dados}

A seguir são apresentados gráficos que demonstram as curvas de crescimento destes dois grupos morfológicos - ao longo de nove dias. Os gráficos foram plotados em função do tempo e número de microrganismos por mililitro de amostra. 0 número de microrganismos em cada ponto do gráfico representa o valor médio das 12 amostras analisadas a cada 24 horas. 


\subsection{Tratamento Estatístico}

O teste de análise de variância - ANOVA (Kruskall Wallis 1) - $\alpha=0,05$, foi aplicado para análise comparativa das dinâmicas de crescimento dos microrgamisnos.

\subsection{Gráficos Experimento I - sulfato de cobre}

Figura 1:

Quantidade de bactérias filamentosas em funçăo do tempo, em substrato com DBO de $350 \mathrm{mg} /$, sem adiçăo de sulfato de cobre.

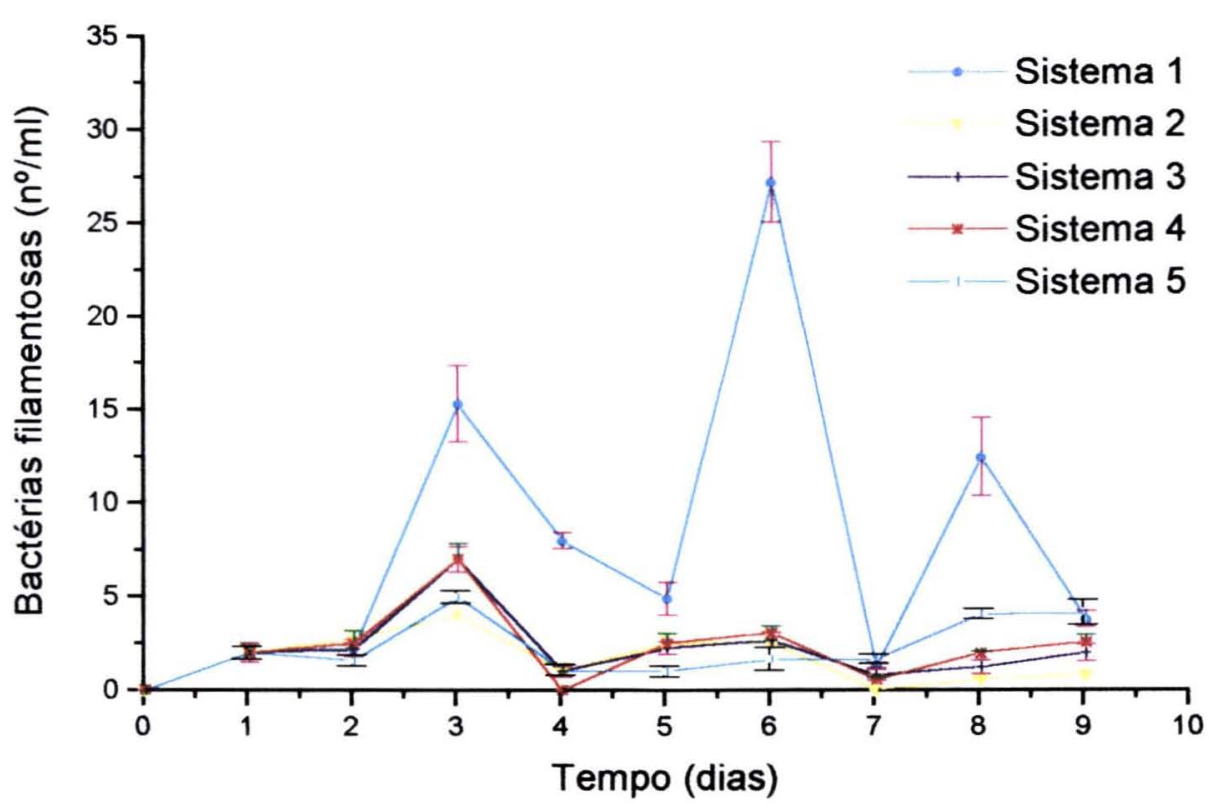


Figura 2:

Quantidade de bactérias filamentosas em função do tempo, em substrato com DBO de $350 \mathrm{mg} / \mathrm{l}$ e adição de 0,78 ppm de cobre no dia 4.

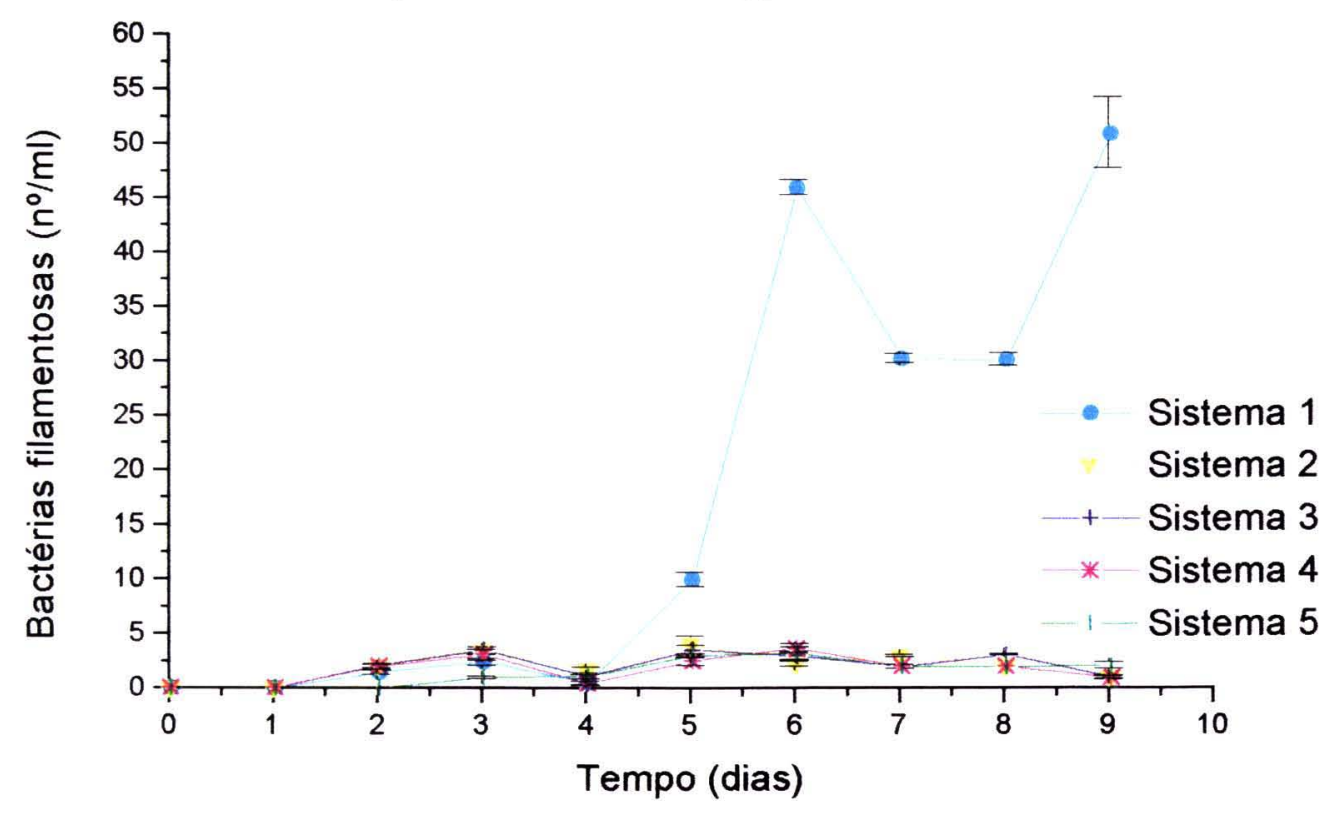

Figura 3:

Quantidade de bactérias filamentosas em funçăo do tempo, em substrato com DBO de 350mg/l e adiçăo de 1,56 ppm de cobre no dia 4.

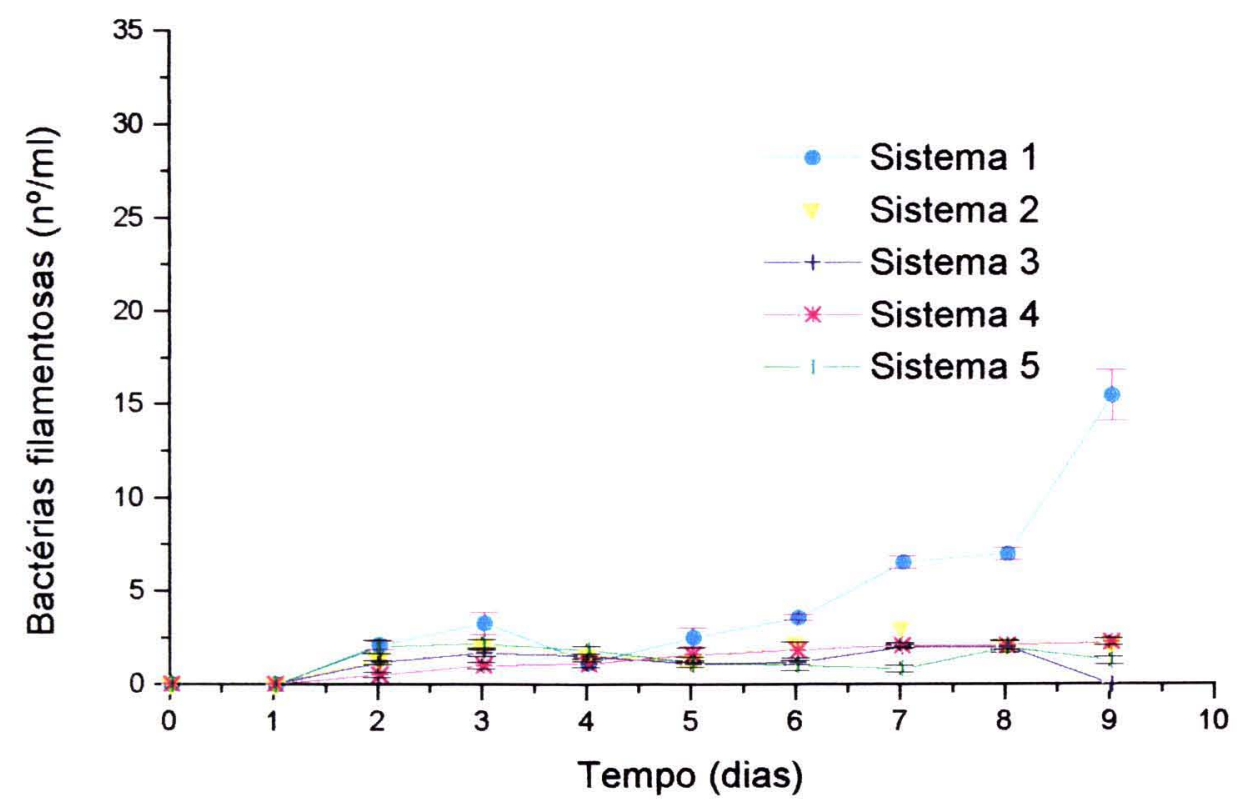


Figura 4:

Quantidade de bactérias filamentosas em função do tempo, em substrato com DBO de 350mg/l e adição de 3,12 ppm de cobre no dia 4 .

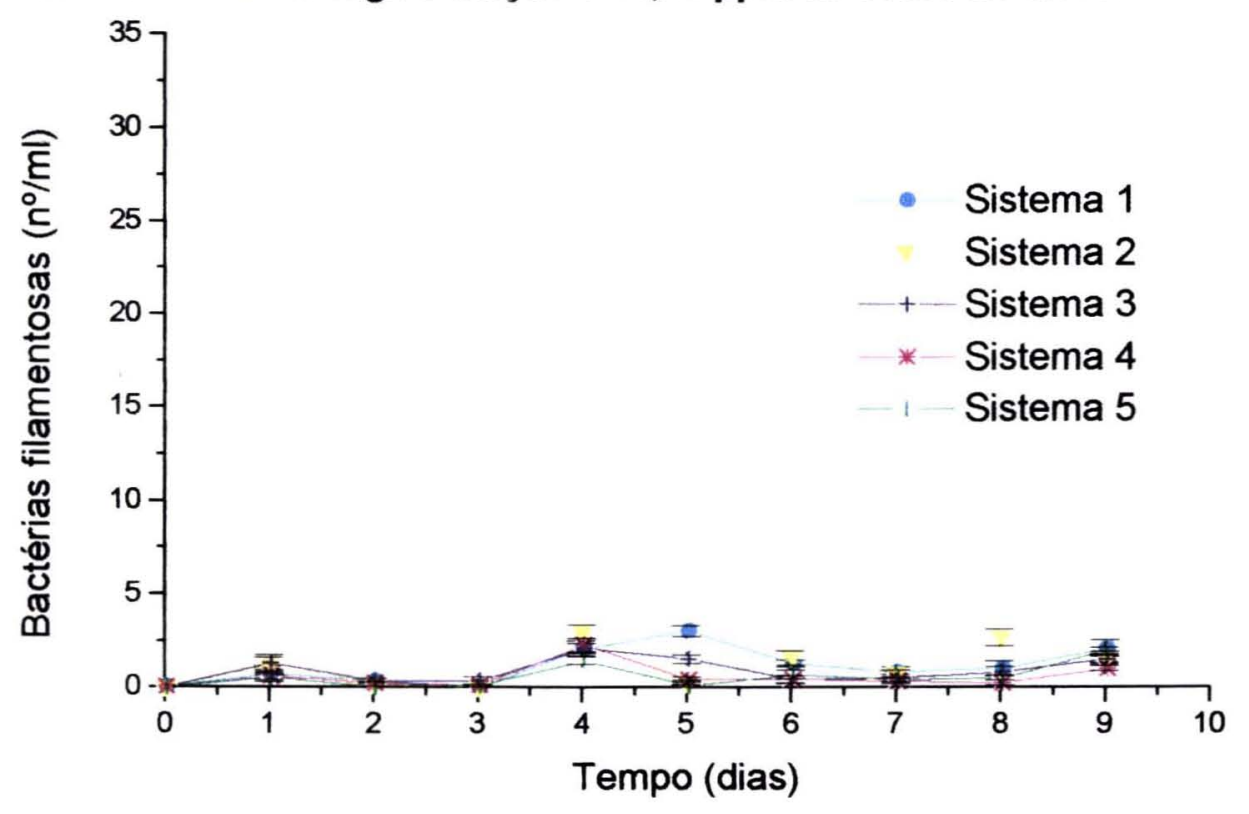

Figura 5:

Quantidade de ciliados em função do tempo, em substrato com DBO de $350 \mathrm{mg} /$, sem adição de sulfato de cobre.

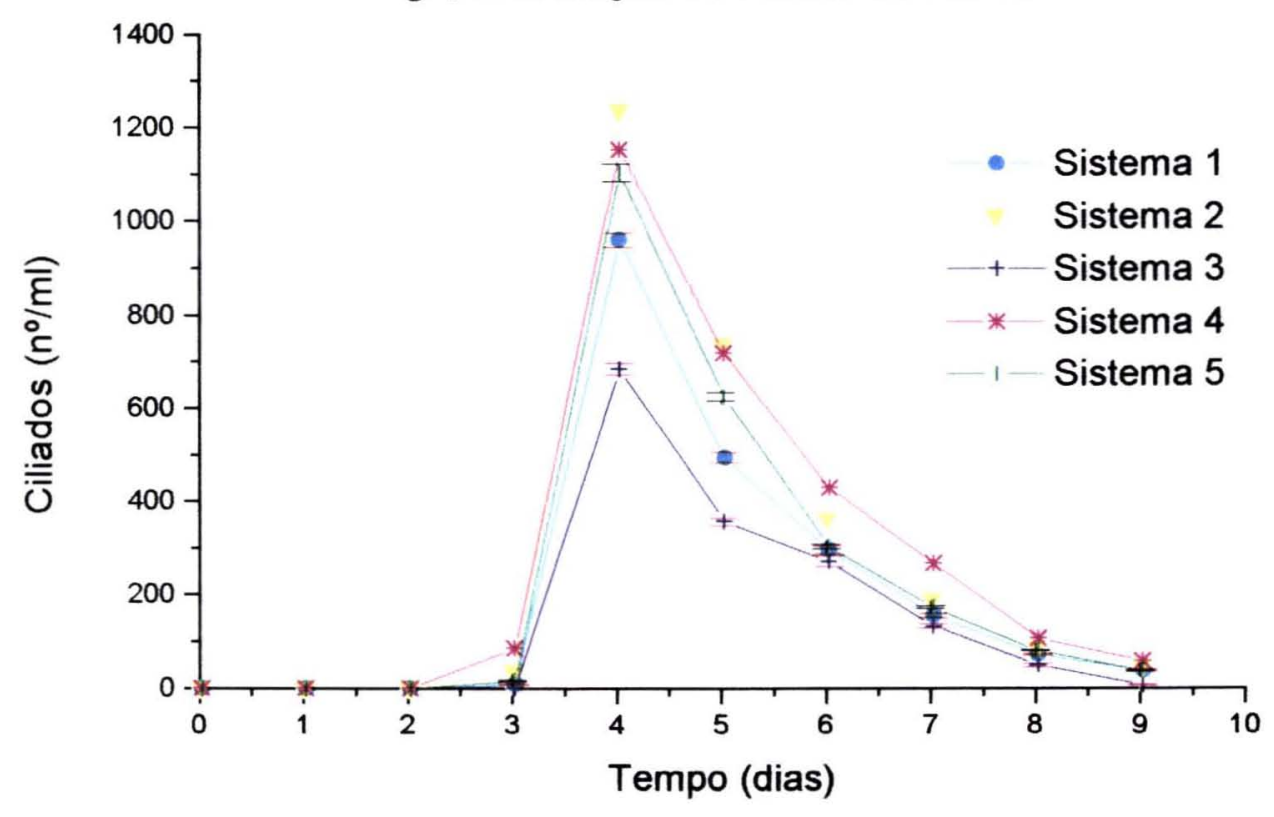


Figura 6:

Quantidade de ciliados em função do tempo, em substrato com DBO de $350 \mathrm{mg} / \mathrm{l}$ e adição de 0,78 ppm de cobre no dia 4.

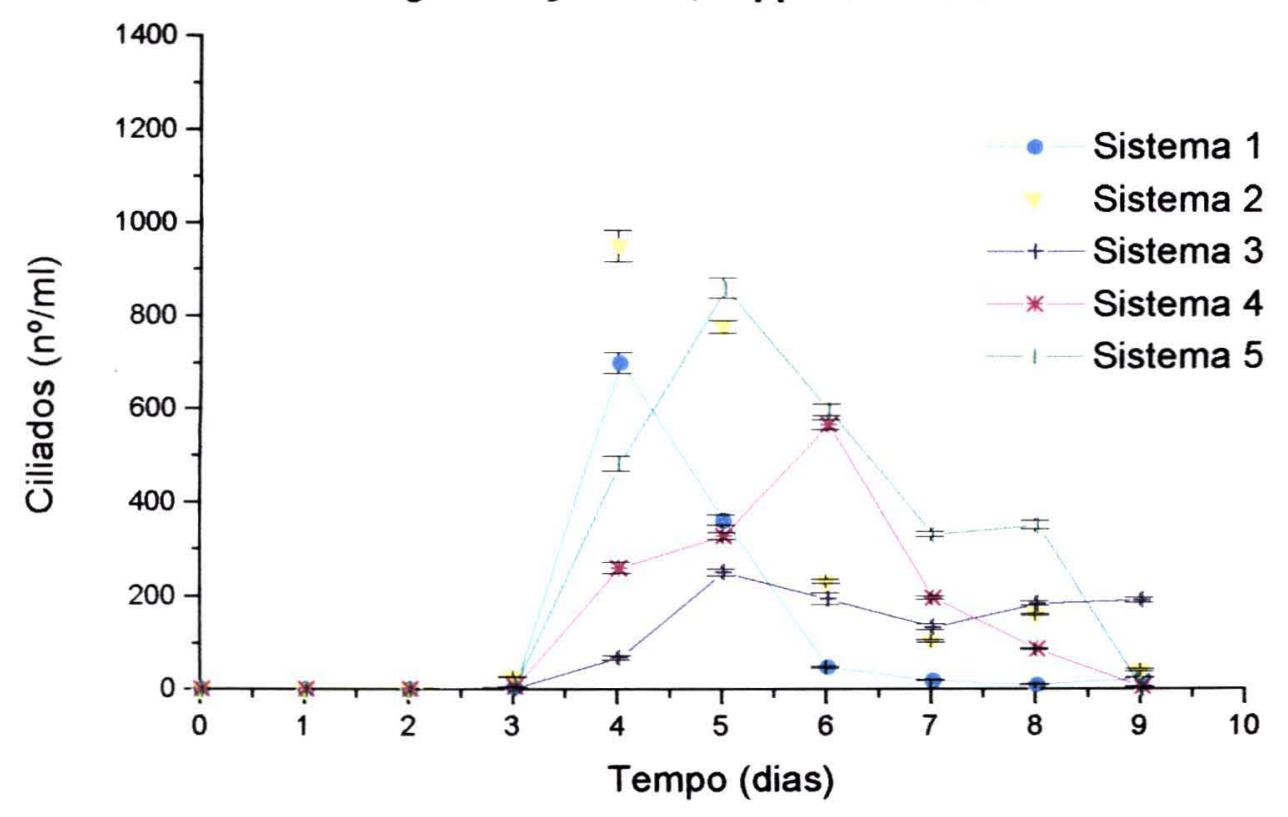

Figura 7:

Quantidade de ciliados em função do tempo, em substrato com DBO de $350 \mathrm{mg} / l$ e adição de 1,56 ppm de cobre no dia 4.

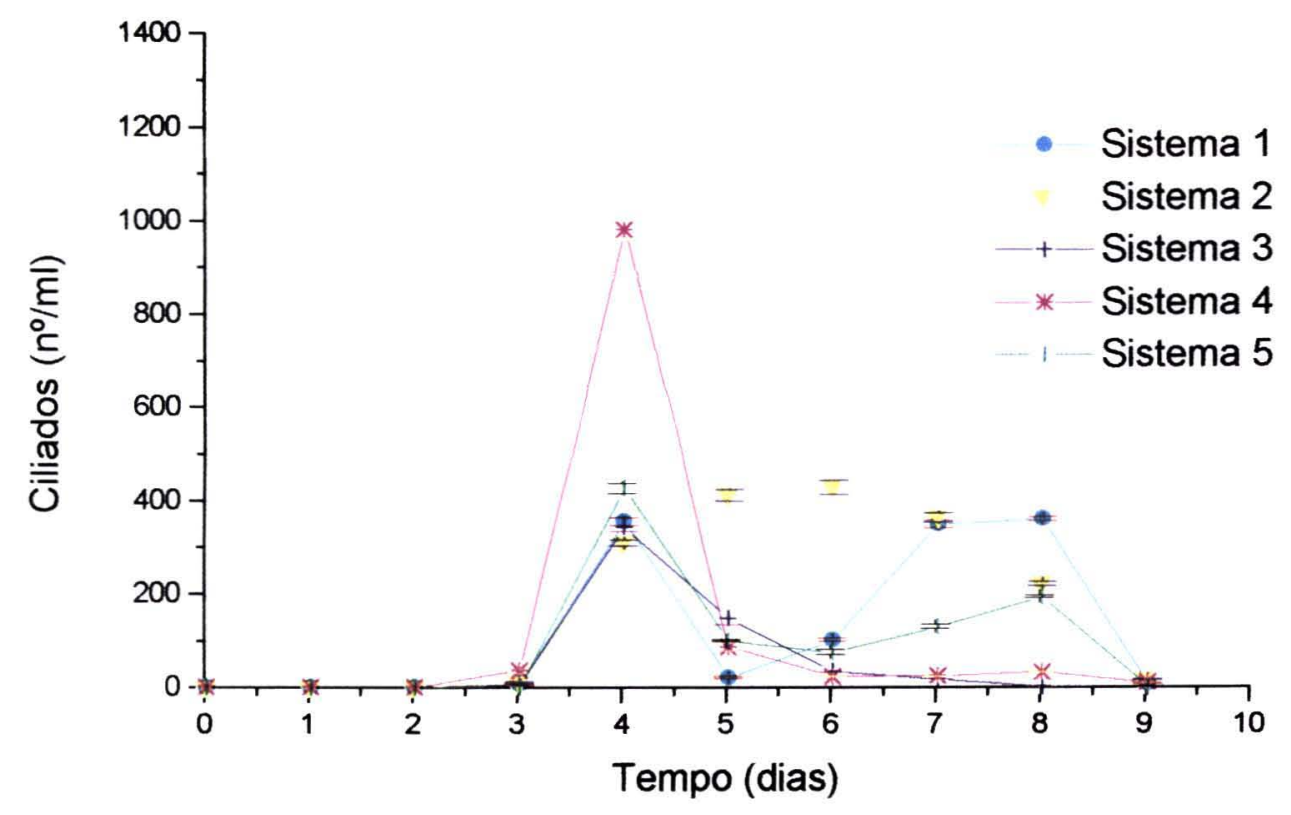


Figura 8:

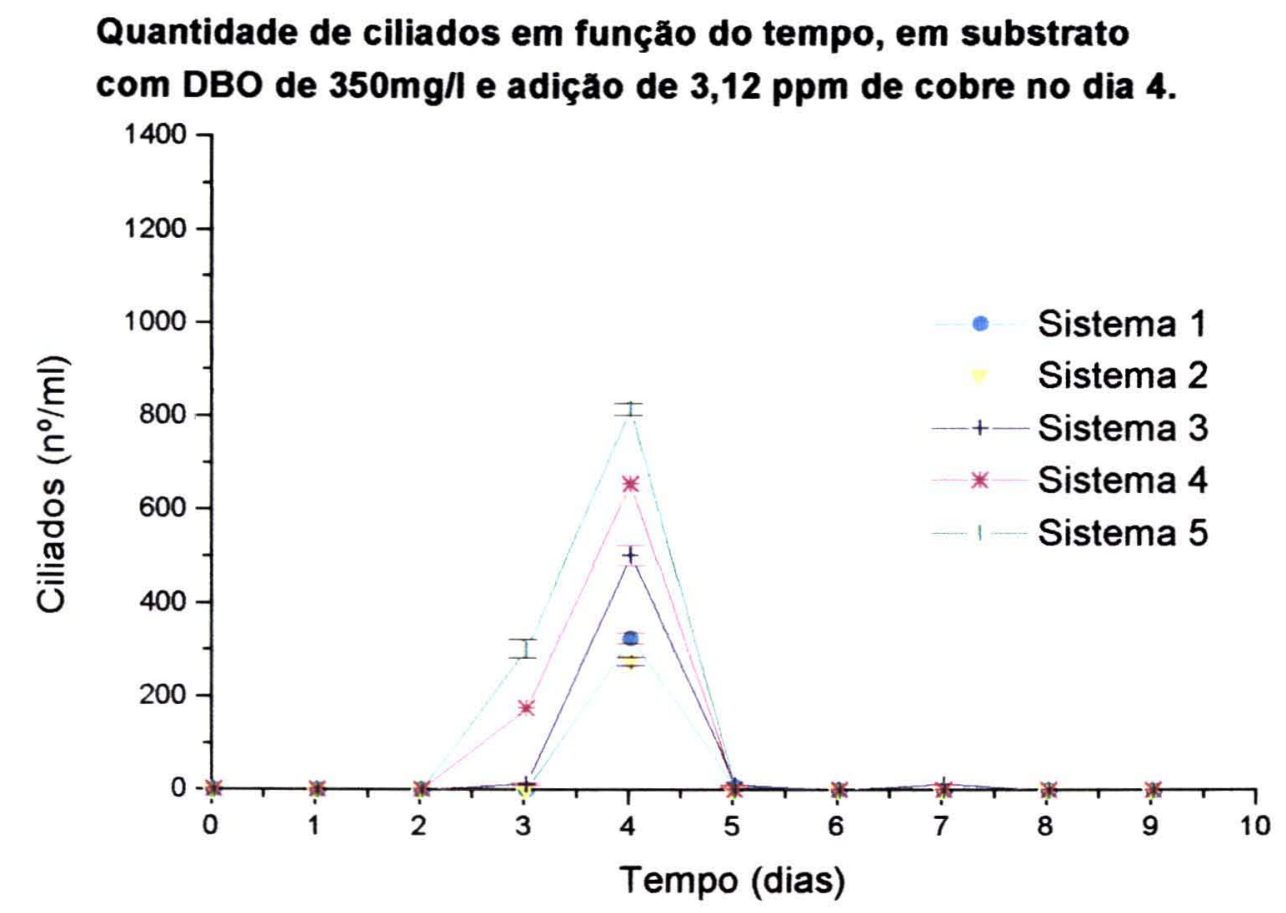

Tabela 2 - Análise de Variância Kruskall-Wallis 1- Experimento I

\begin{tabular}{|c|c|c|c|c|c|c|c|c|c|}
\hline Filamentosas & Xi & $\mathbf{X} 2$ & $\mathrm{X} 3$ & $\mathrm{X} 4$ & $\mathbf{X 5}$ & $\mathbf{X} 6$ & $\mathrm{N7}$ & $\mathrm{X} 8$ & $\mathbf{X 9}$ \\
\hline Microecossistemas replicados & 5 & 5 & 5 & 5 & 5 & 5 & 5 & 5 & 5 \\
\hline Total de microecossistemas & 20 & 20 & 20 & 20 & 20 & 20 & 20 & 20 & 20 \\
\hline Chi-Square & 16,0714 & 12,2600 & 16,4829 & 8,0257 & 9,0200 & 11,8914 & 9,5171 & 5,5000 & 3,6429 \\
\hline Significância & 0,0011 & 0,0065 & 0,0009 & 0,0455 & 0,0290 & 0,0078 & 0,0231 & 0,1386 & 0,3027 \\
\hline Ciliados & M1 & $\mathrm{M} 2$ & M3 & M4 & M5 & M6 & M7 & M8 & $\mathrm{M9}$ \\
\hline Chi-Square & & & 1,4943 & 8,0743 & 14,567 & 12,7143 & 10,9314 & 11,2629 & 12,06000 \\
\hline Significância & & & 0,6836 & $0,0+45$ & 0,0022 & 0,0053 & 0,0121 & 0,0104 & 0,0072 \\
\hline
\end{tabular}




\subsection{Gráficos Experimento II - Sulfato de Cobre}

Figura 9:

Quantidade de bactérias filamentosas em função do tempo, em substrato com DBO de $350 \mathrm{mg} / \mathrm{l}$, sem adição de sulfato de cobre.

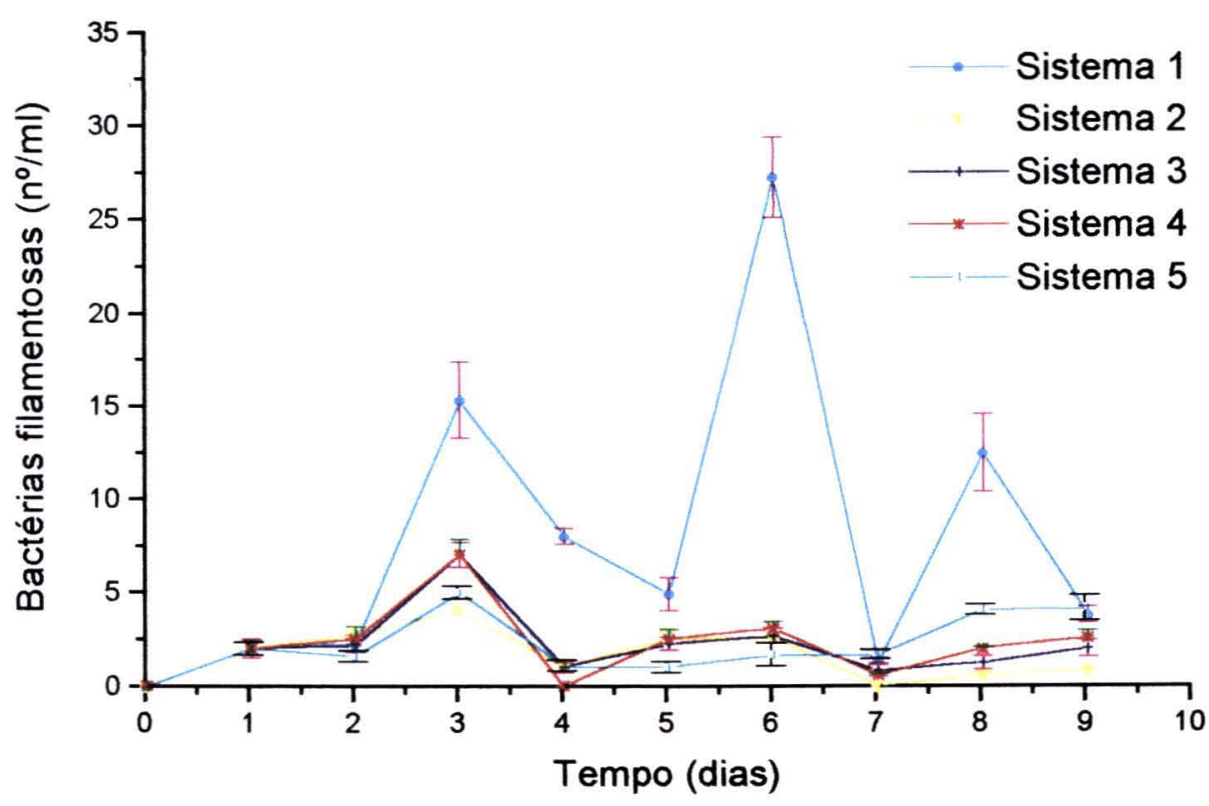


Figura 10:

Quantidade de bactérias filamentosas em função do tempo, em substrato com DBO de $350 \mathrm{mg} / \mathrm{l}$ e adição de 0,78 ppm de cobre no dia 4 .

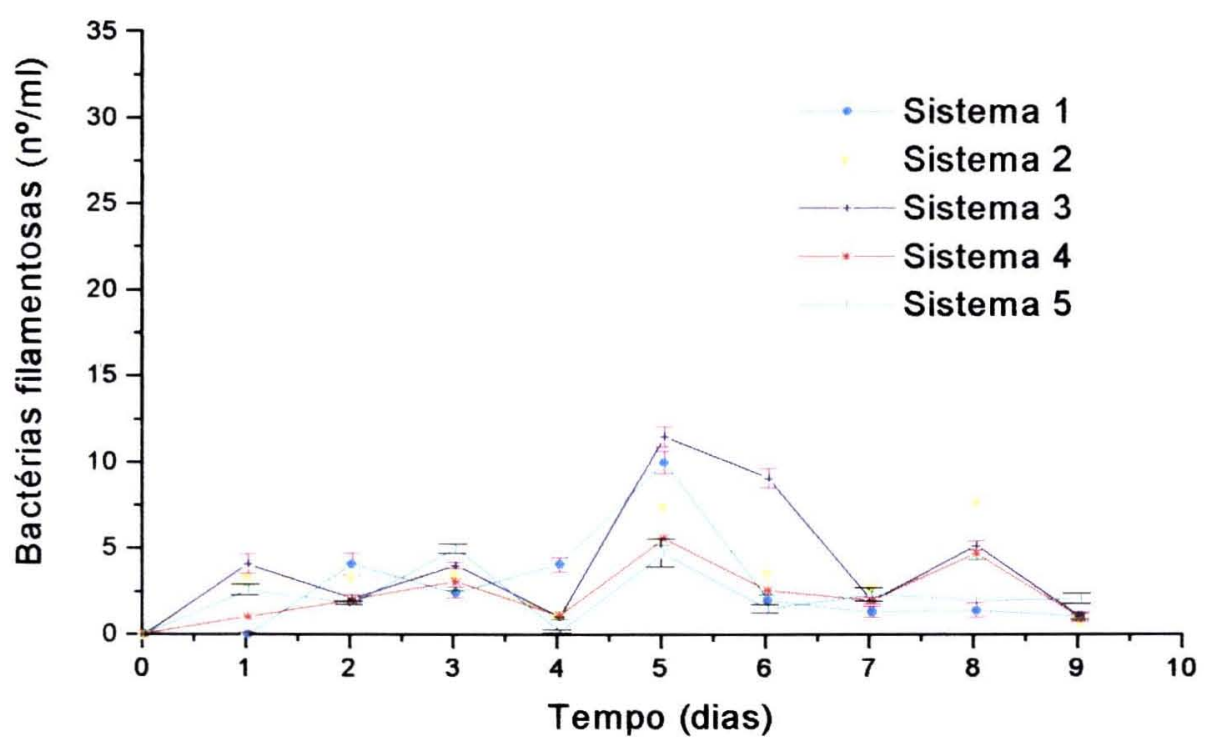

Figura 11:

Quantidade de bactérias filamentosas em função do tempo, em substrato com DBO de $350 \mathrm{mg} / \mathrm{l}$ e adição de 1,56 ppm de cobre no dia 4.

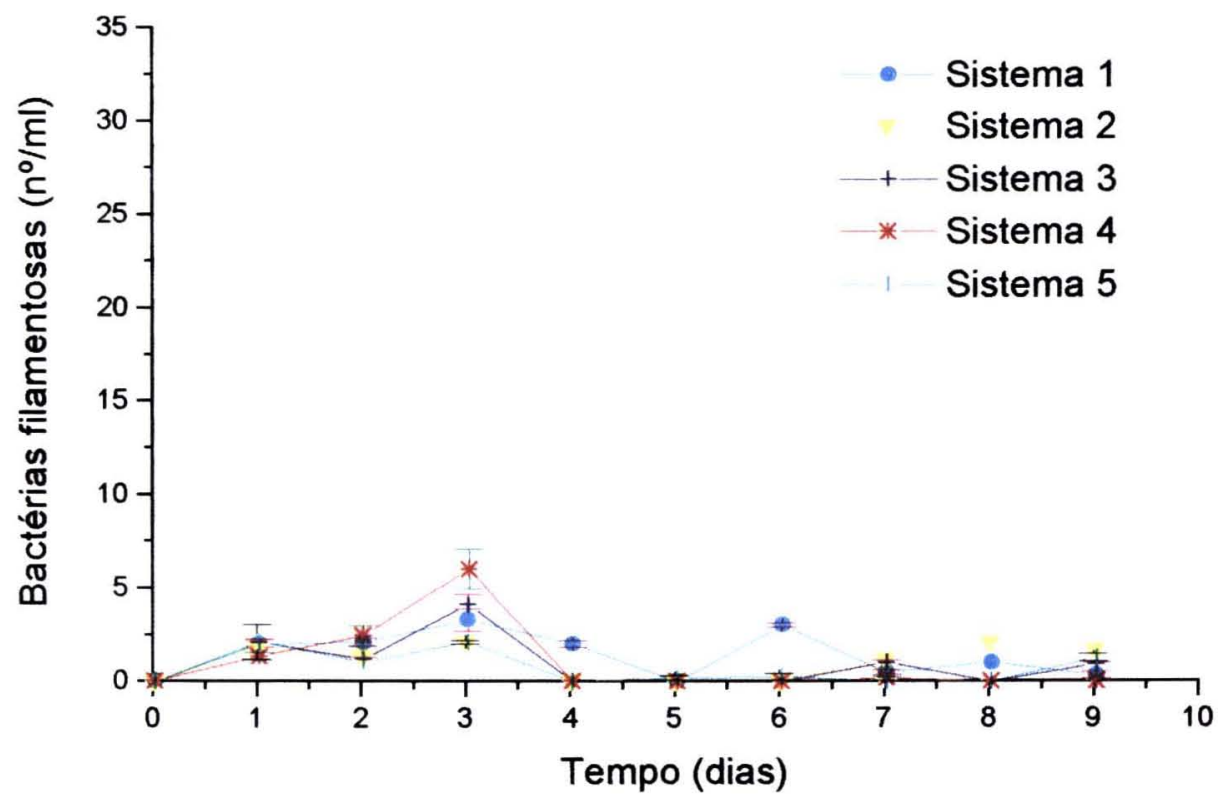


Figura 12:

Quantidade de bactérias filamentosas em função do tempo, em substrato com DBO de 350mg/l e adição de 3,12 ppm de cobre no dia 4 .

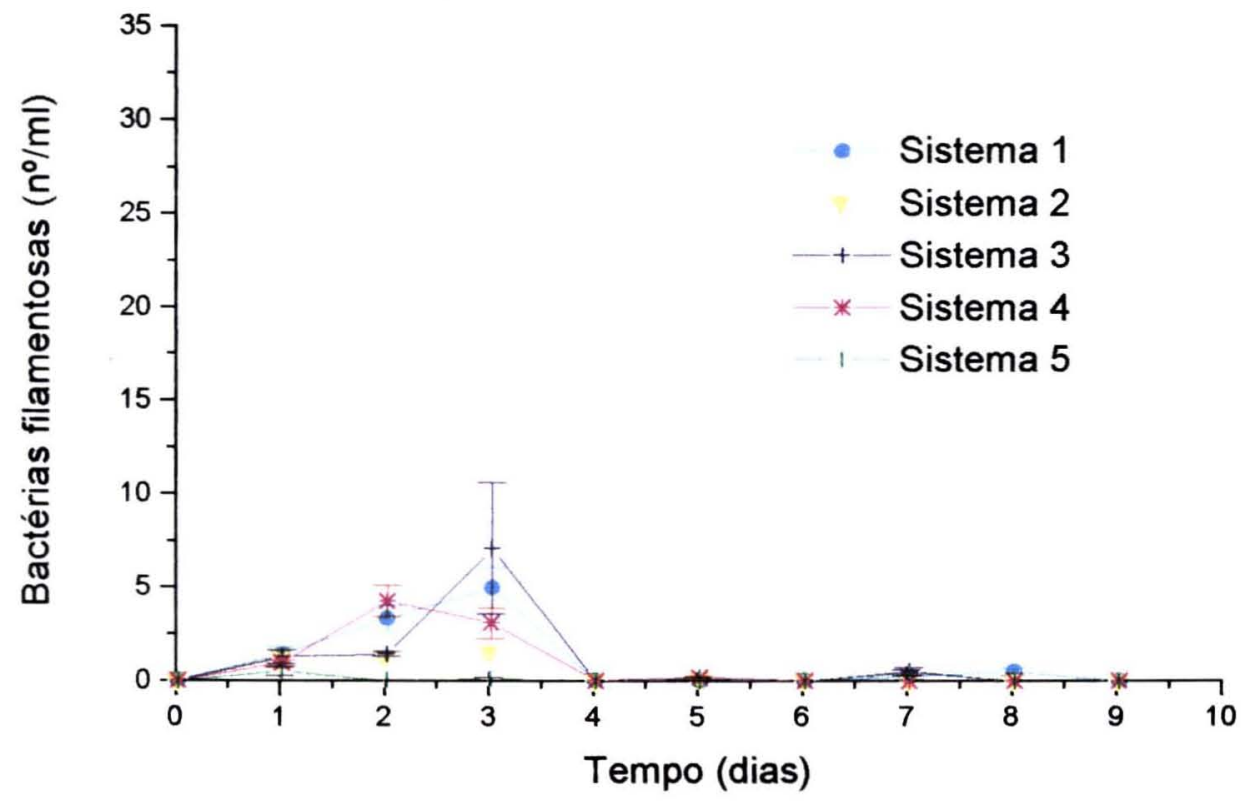

Figura 13:

Quantidade de ciliados em função do tempo, em substrato com DBO de $350 \mathrm{mg} / \mathrm{l}$, sem adição de sulfato de cobre.

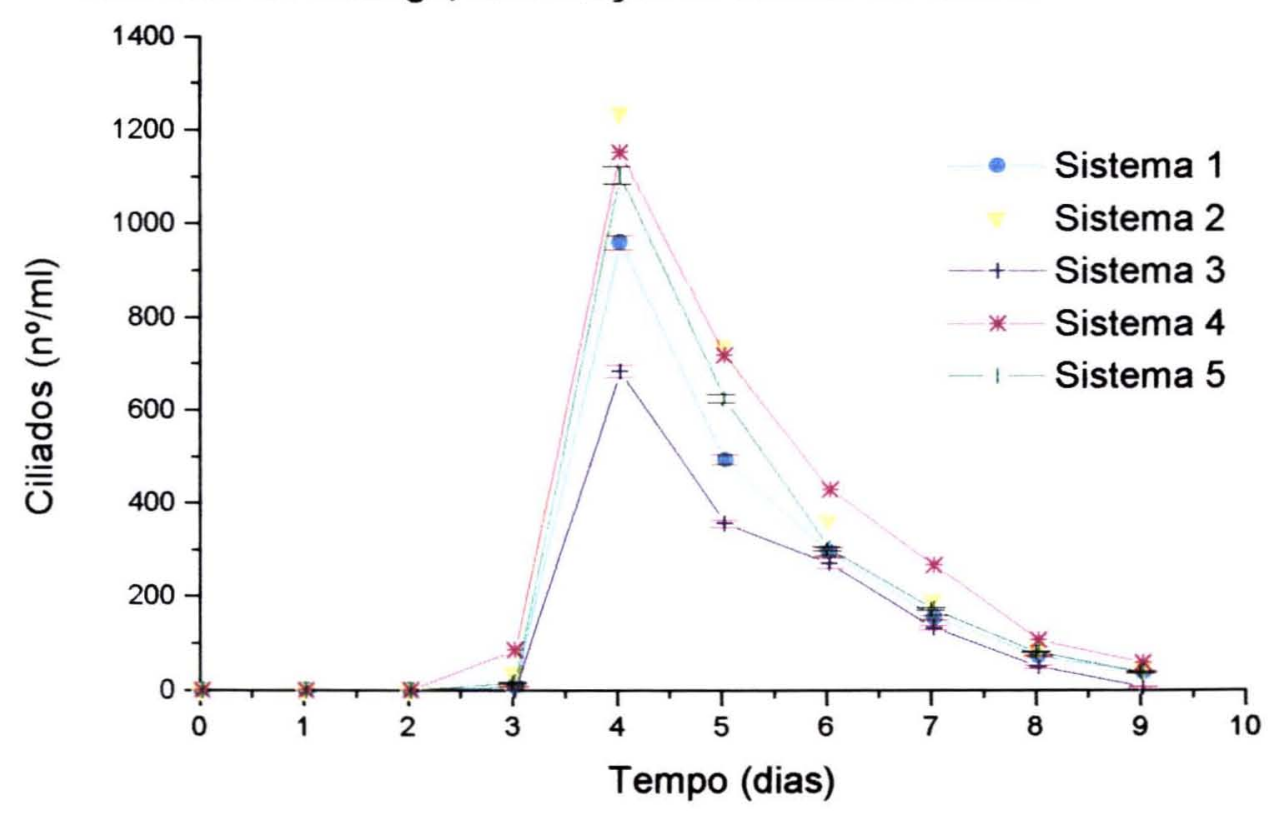


Figura 14:

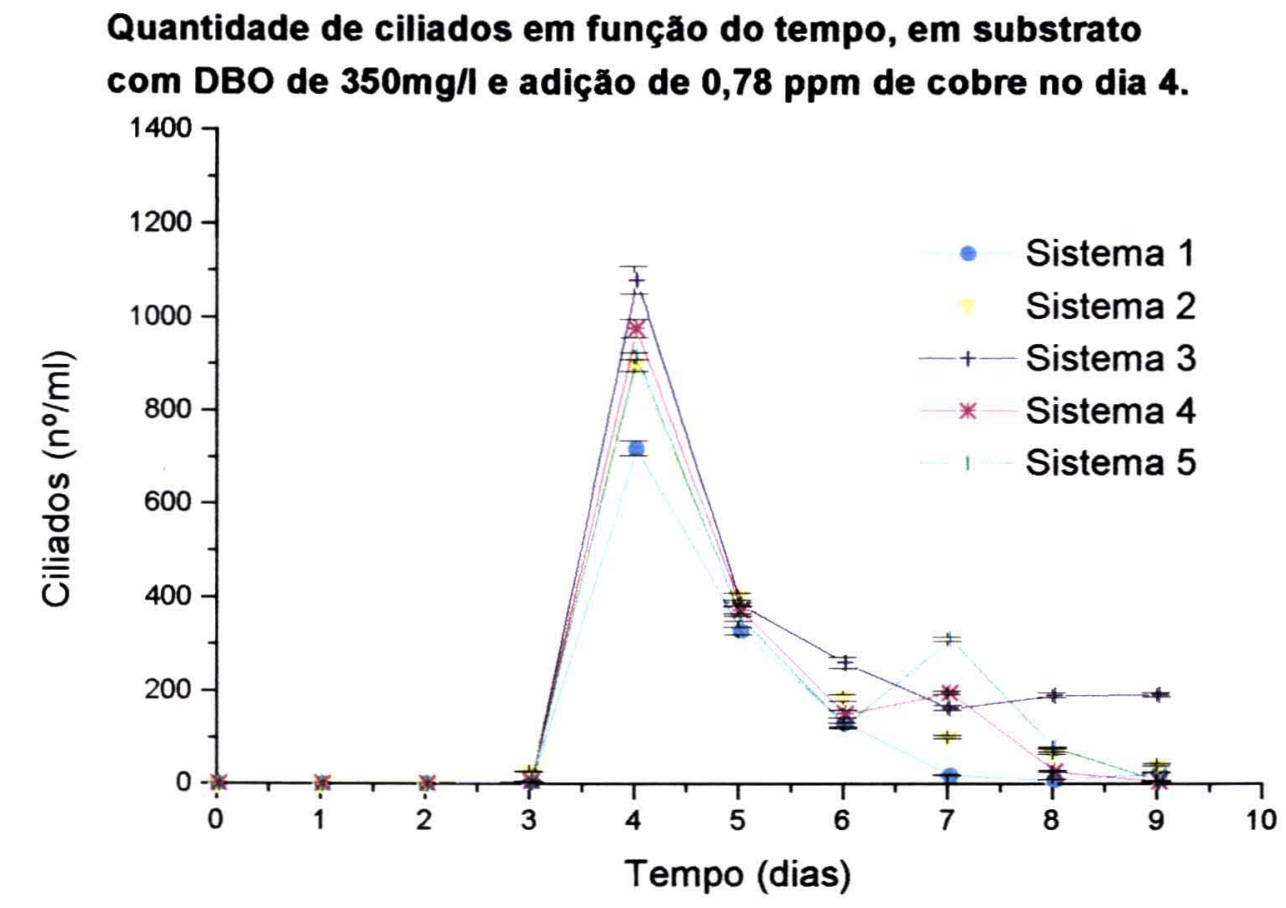

Figura 15:

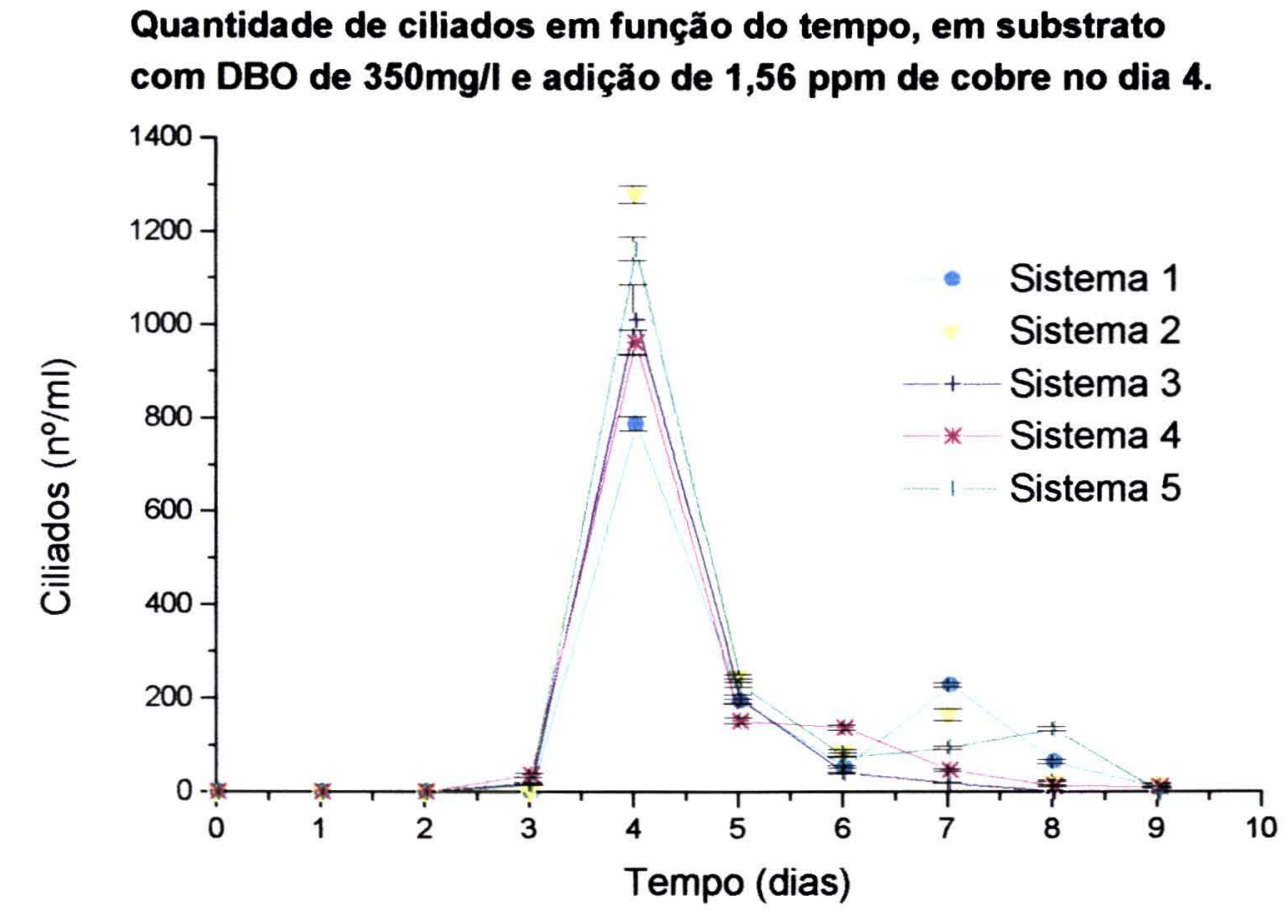


Figura 16:

\section{Quantidade de ciliados em função do tempo, em substrato} com DBO de 350mg/l e adição de 3,12 ppm de cobre no dia 4.

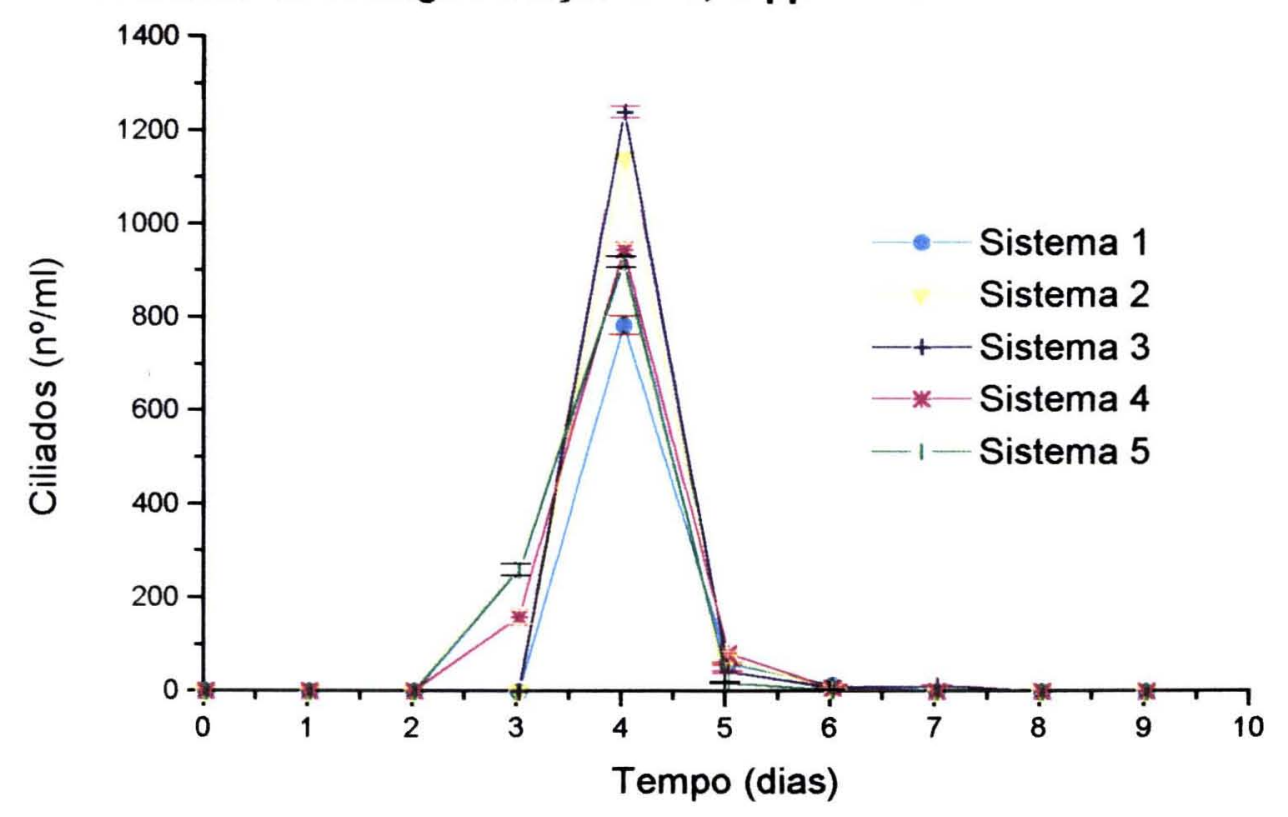

Tabela 3 - $\quad$ Análise de Variância Kruskall-Wallis - Experimento II

\begin{tabular}{|c|c|c|c|c|c|c|c|c|c|}
\hline Filamentosas & $\mathrm{X} 1$ & $\mathrm{X} 2$ & $\mathrm{x} 3$ & $X_{4}$ & $\times 5$ & $\times 6$ & $\times 7$ & $\times 8$ & $\times 9$ \\
\hline Microecossistemas replicados & 5 & 5 & 5 & 5 & 5 & 5 & 5 & 5 & 5 \\
\hline Total de microecossistemas & 20 & 20 & 20 & 20 & 20 & 20 & 20 & 20 & 20 \\
\hline Chi-Square & 9,1486 & 3,2429 & 5,5743 & 8,6429 & 15,4114 & 12,4457 & 10,8086 & 11,9857 & 12,5800 \\
\hline Significância & 0,0274 & 0,3557 & $0,13+3$ & 0,0344 & 0,0015 & 0,006 & 0,0128 & 0,0074 & 0,0056 \\
\hline Ciliados & $M 1$ & $\mathrm{M12}$ & M3 & $M 4$ & M5 & M6 & M7 & M8 & 19 \\
\hline Chi-Square & & & 0,7629 & 2,1771 & 17,1029 & 17,331 & 11,6743 & 12,0971 & 0,0072 \\
\hline Significância & & & 0,8583 & 0,5365 & 0,007 & 0,006 & 0,0086 & 0,0071 & 0,0091 \\
\hline
\end{tabular}




\subsection{Experimento III - Inseticida DDVP 20}

Figura 17:

Quantidade de bactérias filamentosas em função do tempo em substrato com DBO de $350 \mathrm{mg} /$, sem adição de inseticida.

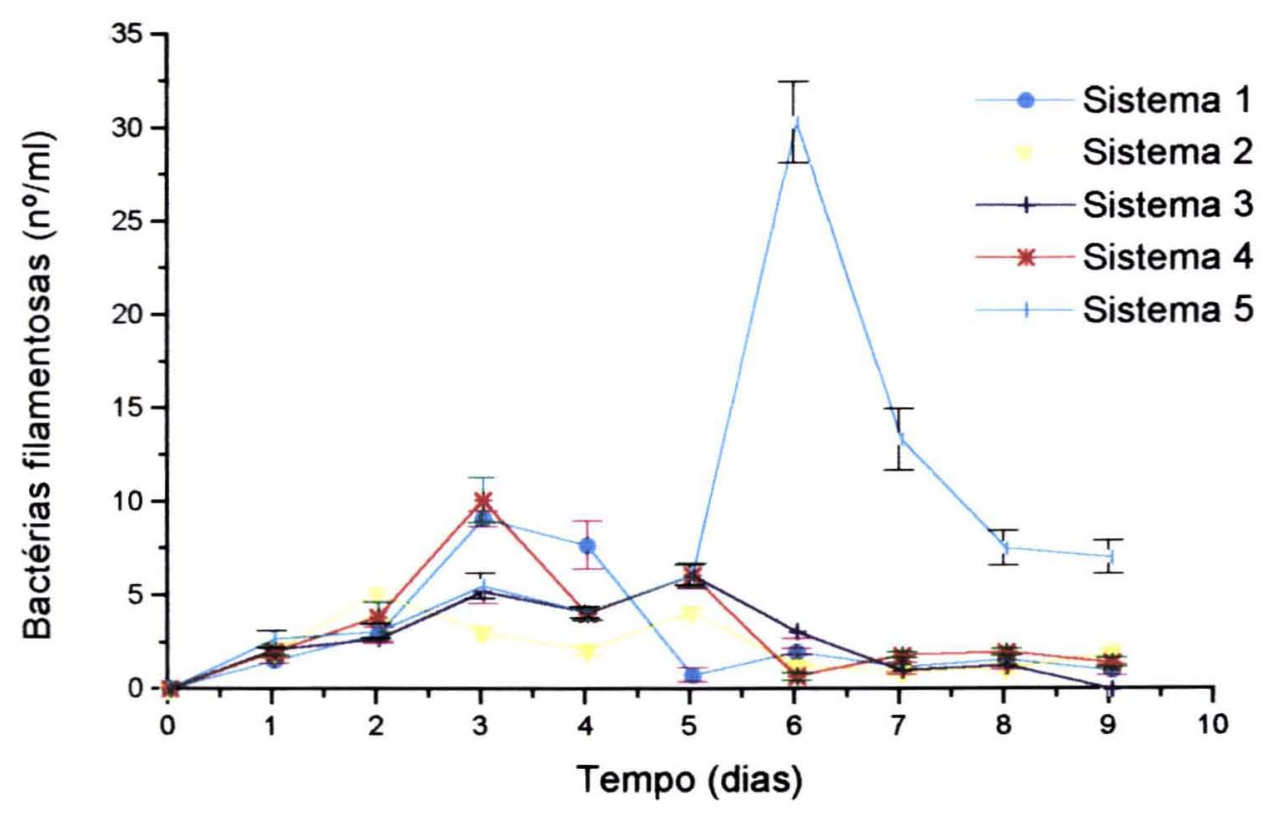


Figura 18:

Quantidade de bactérias filamentosas em função do tempo, em substrato com DBO de $350 \mathrm{mg} / /$ e adição de $0,62 \mathrm{ml} / \mathrm{l}$ de inseticida DDVP 20 no dia 4.

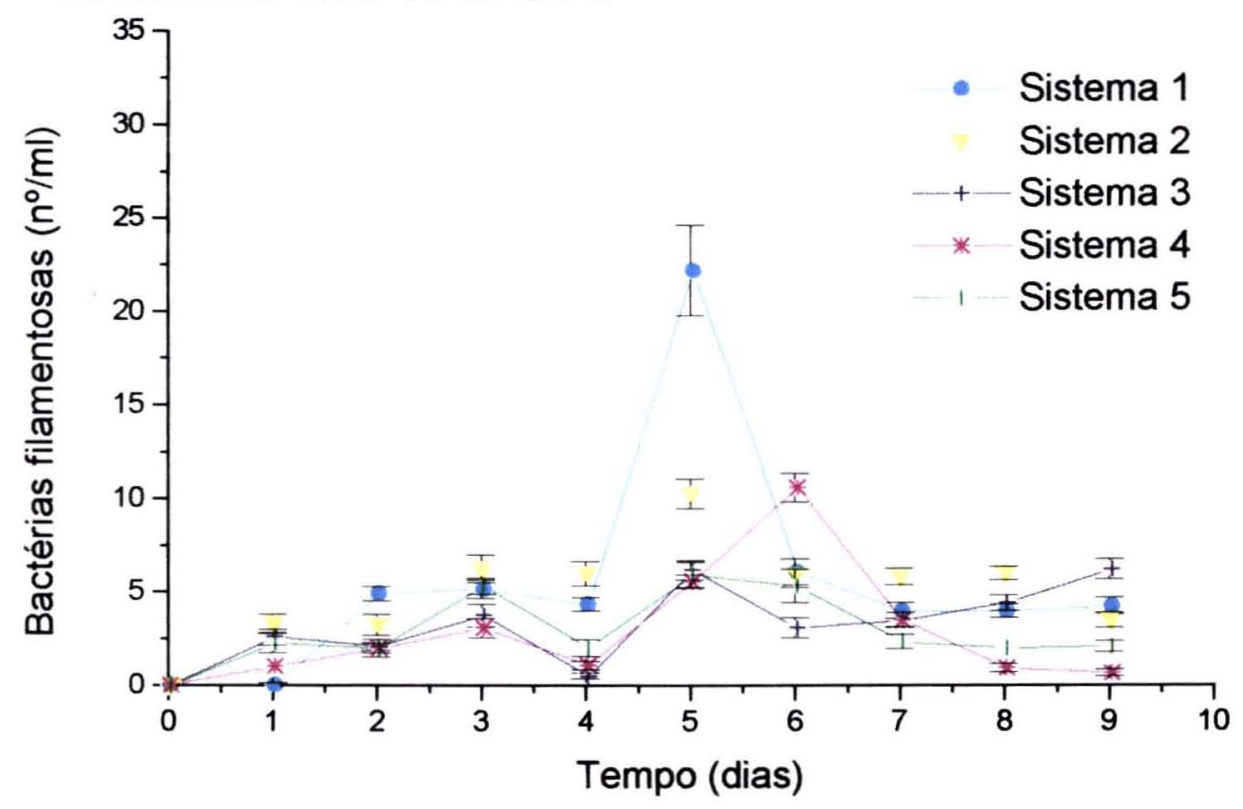

Figura 19:

Quantidade de bactérias filamentosas em função do tempo, em substrato com DBO de $350 \mathrm{mg} / /$ e adição de $1,25 \mathrm{ml} / \mathrm{l}$ de inseticida DDVP 20 no dia 4.

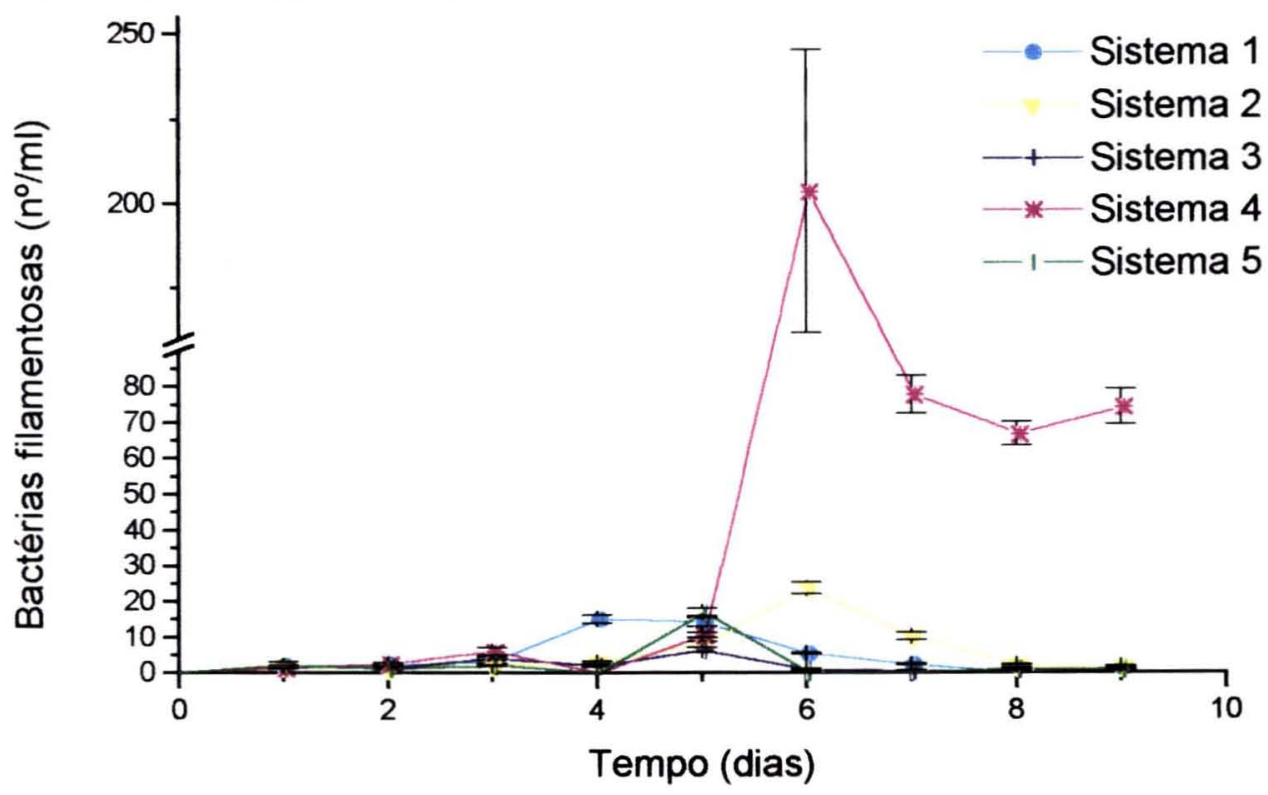


Figura 20:

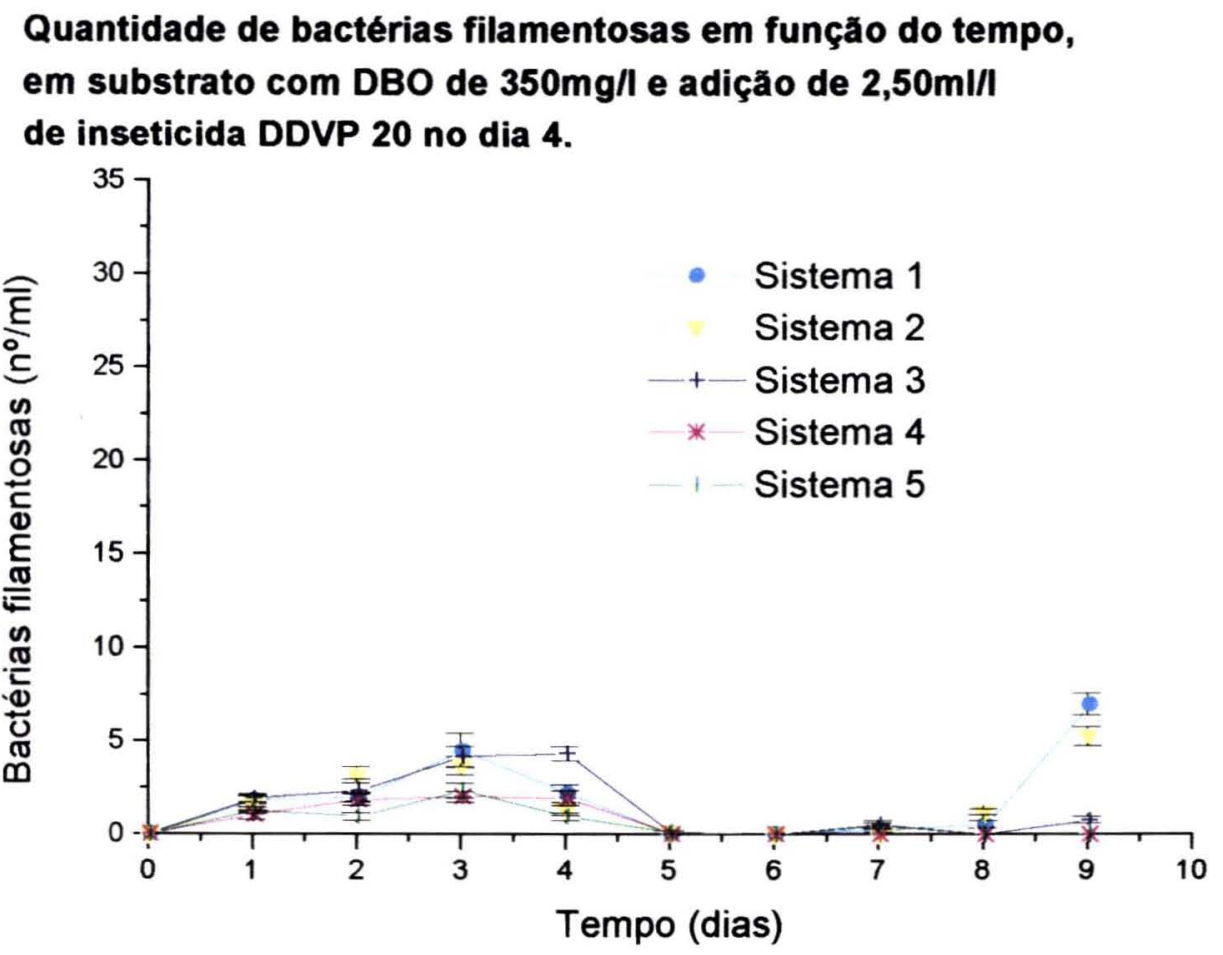

Figura 21:

Quantidade de ciliados em função do tempo, em substrato com DBO de $350 \mathrm{mg} / \mathrm{l}$ sem adição de inseticida.

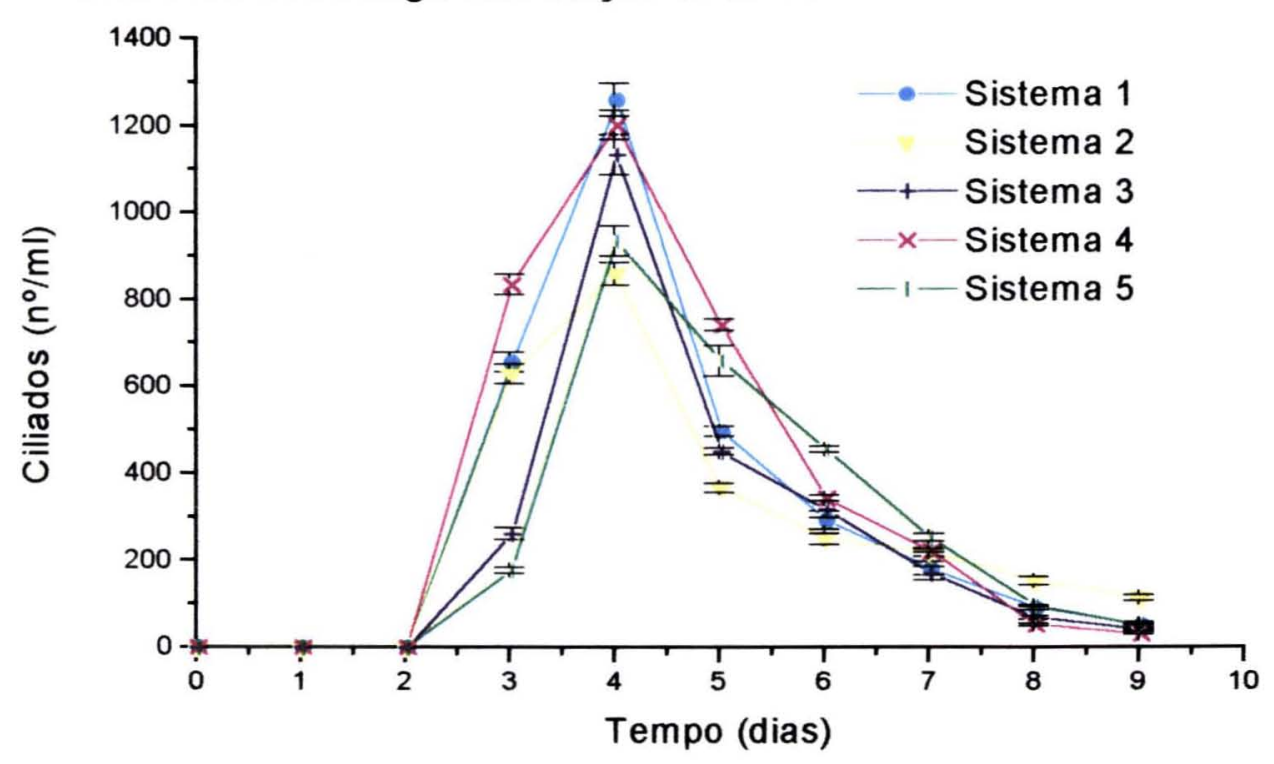


Figura 22:

Quantidade de ciliados em função do tempo em substrato com DBO de $350 \mathrm{mg} / /$ e adição de $0,62 \mathrm{mI} / /$ de inseticida DDVP 20 no dia 4.

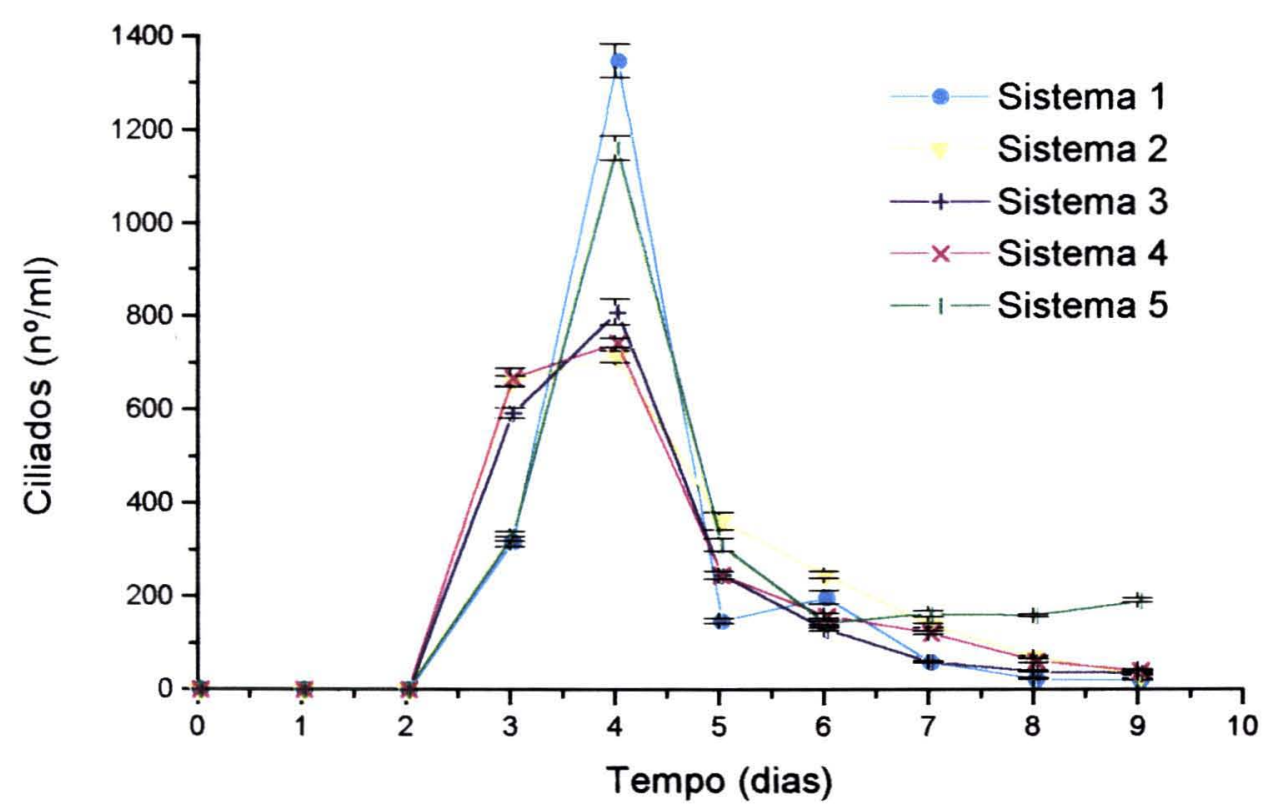

Figura 23:

Quantidade de ciliados em função do tempo em substrato com DBO de $350 \mathrm{mg} / \mathrm{l}$ e adição de $1,25 \mathrm{~m} / / \mathrm{l}$ de inseticida DDVP 20 no dia 4 .

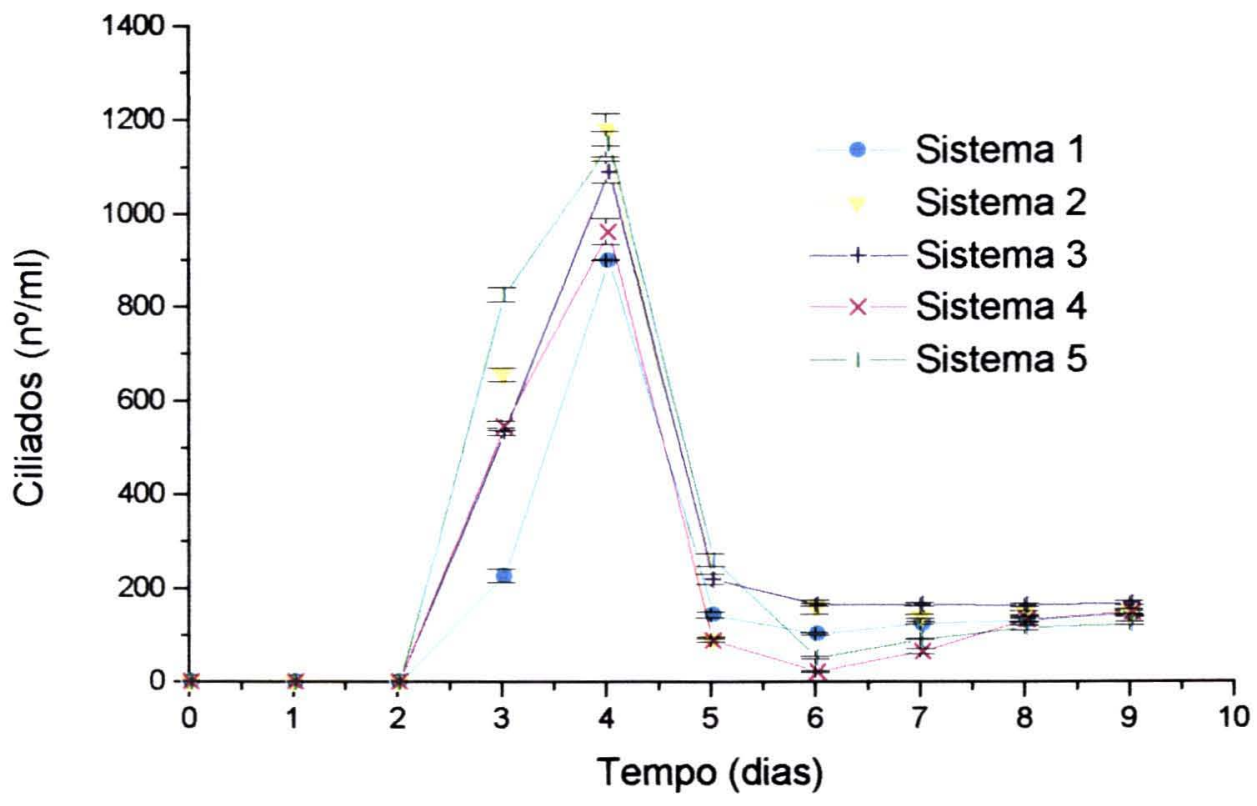


Figura: 24

\section{Quantidade de ciliados em substrato com DBO de $350 \mathrm{mg} / /$ e adição de 2,50mI/I de inseticida DDVP 20 no dia 4.}

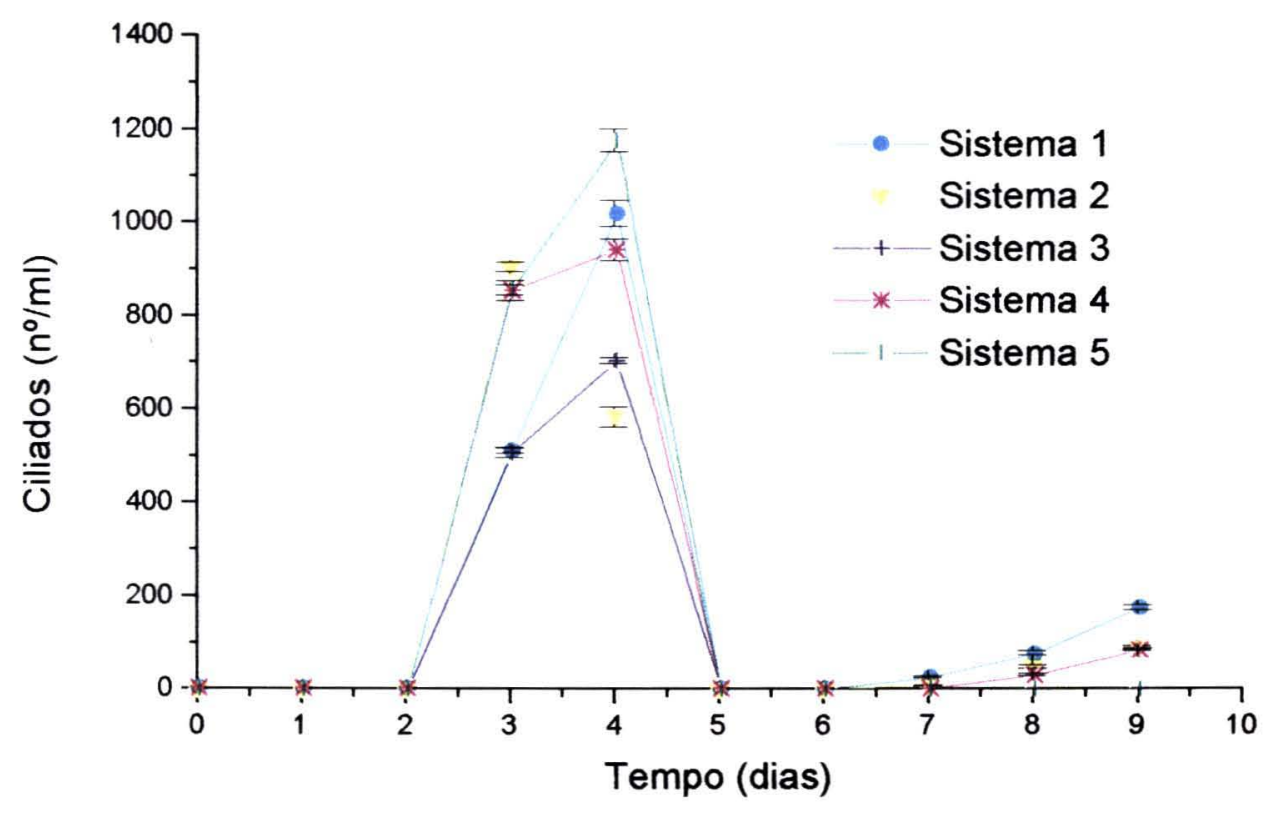

Tabela 4 - $\quad$ Análise de Variância Kruskall-Wallis 1 - Experimento III

\begin{tabular}{llllllllll}
\hline Filamentosas & $\mathbf{X 1}$ & $\mathbf{X 2}$ & $\mathbf{X 3}$ & $\mathbf{X 4}$ & $\mathbf{X 5}$ & $\mathbf{X 6}$ & $\mathbf{X} 7$ & $\mathbf{X 8}$ & X9 \\
\hline Microecossistemas replicados & 5 & 5 & 5 & 5 & 5 & 5 & 5 & 5 & 5 \\
\hline Total de microecossistemas & 20 & 20 & 20 & 20 & 20 & 20 & 20 & 20 & 20 \\
\hline Chi-Square & 3,4143 & 8,0886 & 5,5800 & 2,7971 & 13,6400 & 10,8829 & 9,5743 & 9,1971 & 1,1571 \\
\hline Significância & 0,3321 & 0,0442 & 0,1339 & 0,424 & 0,0034 & 0,0124 & 0,0226 & 0,268 & 0,7633 \\
\hline & & & & & & & & & \\
\hline Ciliados & M1 & $\mathbf{M} 2$ & $\mathbf{M} 3$ & M4 & M5 & M6 & M7 & M8 & M9 \\
\hline Chi-Square & & & 1,8800 & 2,2914 & 17,1029 & 16,5543 & 16,2114 & 10,1771 & 5,7886 \\
\hline Significâncial & & & 0,5977 & 0,5142 & 0,0007 & 0,0009 & 0,001 & 0,0171 & 0,1224 \\
\hline
\end{tabular}




\section{DISCUSSÃO}

O modelo experimental foi capaz de constituir um microecossistema aquático com uma comunidade de microrganismos típica a partir de simples aeração de leite desnatado diluído em água destilada com Demanda Bioquímica de Oxigênio de $350 \mathrm{mg} / \mathrm{l}$.

Com a metologia utilizada foi possível identificar dois grupos morfológicos distintos que se desenvolvem seqüêncialmente dois quais, bactérias filamentosas e protozoários do tipo ciliados livres.

A geração de bactérias filamentosas foi observada a partir de 24 horas de aeração. A geração dos protozoários ocorre por volta de 72 horas do início do experimento. Os gráficos apresentam as curvas de crescimento dos dois grupos distintamente.

Observa-se que a média dos números de filamentos foram bastante inexpressivos em geral de 2 a 10 unidades em todos microecossistemas nos três experimentos. Sendo que cada fragmento foi considerado uma unidade. Os números são bastante oscilantes ora diminuindo ora aumentado tanto nos sistemas controle quanto nos sistemas contendo as substâncias tóxicas, isto é, o sultafo de cobre e o inseticida DDVP nas várias concentrações aplicadas.

Não foi observada relação direta na inibição do crescimento de bactérias pela introdução dos tóxicos. Mas, foram observados, alguns picos de crescimento, atingindo médias de 30 filamentos em alguns microecossistemas e alguns dos quais sob ação dos tóxicos - sistema 1 - experimento I (figura 1); sistema 1 - experimento II (figura 9) e sistema 1 - experimento III (figura 17). 
Por observação qualitativa, a variabilidade numérica para bactérias filamentosas supera a ação de ambos os tóxicos, com exceção da concentração de $12,28 \mathrm{mg} / \mathrm{l}$ de cobre (3,12 ppm), momento em que há inibição total do crescimento sem recuparação nos dias subseqüêntes - sistemas de 1 a 5 - experimento II (figura 12). Recuperação estatísticamente não significativa para os microecosistemas 1 a 5 - Experimento I (figura 4).

O valor referência para a análise de variância entre os sistemas é $\alpha=5 \%$. Sendo que, índices de significância maiores ou iguais a 0,05 demonstrariam que as dinâmicas de crescimentos nos microessistemas, são diferentes em cada momento (cada momento entendese cada dia amostrado). Índices de significância menores ou iguais a 0,05 , demonstram por sua vez, que as dinâmicas dos microecossistemas amostrados são diferentes.

Os valores de dinâmica de crescimento das bactéricas filamentosas no primeiro dia de amostragem são iguais. Nos demais momentos os índices de significância demonstram que as dinâmicas de crescimento são diferentes. Este resultado, demonstra, portanto, que não houve comportamento típico de crescimento para este grupo. O que torna dificil a análise de desequilíbrios provocados pelos agentes tóxicos. Além disso, este resultado acena para a existência de uma variabilidade nos microecossistemas, a qual pode ser observada qualitativamente, por simples análise dos gráficos.

Em contraste. observa-se após 96 horas de aeração, entre o terceiro e quarto dias, que o grupo de ciliados apresenta crescimento exponencial. Os índices de significância demonstram que as dinâmicas são iguais em todos os microecossistemas até este momento do experimento, demonstrando comportanto típico. 
Em termos numéricos a média no quarto dia variou de 500 a 1200 ciliados $/ \mathrm{ml}$. A fase de declínio pode ser observada a partir do quinto dia. O efeito inibidor do sulfato de cobre é observado na concentração $12,28 \mathrm{mg} / \mathrm{l}$ ou $3,12 \mathrm{ppm}$ de cobre - sistemas de 1 a 5 experimento 1 (figura 8) e sistemas de 1 a 5 - experimento II (figura 16).

O efeito inibidor do DDVP no crescimento dos ciliados é observado na concentração de $2,5 \mathrm{ml} / \mathrm{l}$ e parece ser temporário havendo alguma recuperação à partir do sétimo dia sistemas de 1 a 5 - experimento III (figura 24). Os índices de significância para os dias subsequiêntes à aplicação dos tóxicos, demonstram que não houve comportamento padrão no decaimento numérico entre os vários sistemas.

Os resultados com sulfato de cobre são significativos quando comparados com estudos em modelos de microecossistemas aquáticos apresentados por CAIRNS (1974) e BOUDOU e RIBEYRE (1988).

As temperaturas nos sistemas foram satisfatórias nas marcas de 20 a $24^{\circ} \mathrm{C}$. De acordo com a literatura, temperaturas acima de 35 graus poderiam ocasionar desnaturação e queda na taxa de crescimento. Os valores de $\mathrm{pH}$ indicam níveis de neutralidade e portanto satisfatórios. Valores de pH abaixo de 4,0 e acima de 9,5 podem desencadear efeito tóxico (MCKNNEY 1962). Os valores de oxigênio dissolvido encontravam-se satisfatórios entre 5,2 e 6,2 mg/l.

A variabilidade que ocorre em microecossistemas em condições experimentais similares tem sido objeto central nos estudos de desenvolvimento de modelos de sistemas ecológicos. Para BOUDOU E RIBEYRE (1988) a variabilidade reduziria a habilidade de demonstração dos efeitos e sugerem que análises qualitativas são mais indicadas. 


\section{CONCLUSÕES}

O modelo experimental proporcionou ambiente sustentável capaz de gerar uma comunidade microbiológica característica composta por bactérias filamentosas e ciliados. Efeitos inibidores do sulfato de cobre na concentração de $12,28 \mathrm{mg} / \mathrm{l},(3,12 \mathrm{ppm})$ e $2,5 \mathrm{ml} / 1$ de inseticidade DDVP foram demonstrados no crescimento de ciliados, a partir do quarto dia de experimento. Os resultados não foram conclusivos para o grupo de bactérias filamentosas.

O grupo ciliados apresentou dinâmica de crescimento caracterizada por fase nítida de multiplicação logaritmica. Tanto análises qualitativas quanto os índices de significância do teste de aná lise de variância demonstram homogeneidade na dinâmica de ciliados até o quarto dia. A variablidade nas curvas de crescimentos tanto de bactérias quanto para ciliados, sugere análise de tipo qualitativa dos resultados e, conforme apresentado na literatura, a capacidade de auto-organização própria de sistemas ecológicos.

A metodologia permitiu o estudo dos efeitos dos tóxicos para cada grupo de microrganismos gerados em sequêencia ecológica e não forneceu informações que permitam o estudo das relações ecológicas que se operam.

Como sistema fechado o modelo não corresponde à dinâmica dos ecossistemas de modo que seus resultados podem ser comparados somente à amostras pontuais de ecossistemas aquáticos naturais.

O modelo apresenta a vantagem de ser fácilmente montado em laboratório podendo constituir um excelente microecossistema voltado ao estudo dos processos e teorias ecológicas se aliado à técnicas mais sofiscadas de microbiologia ambiental entre outras. 
A DBO do substrato de $350 \mathrm{mg} / \mathrm{l}$ é bastante próxima à de despejos orgânicos comumente lançados no meio aquático, porém como sistema de testes para avaliação de contaminação por substâncias tóxicas, o método carece de estudos aprofundados de parâmetros pertinentes.

A aplicação prática do modelo motivou um tempo de duração do experimento bastante breve, quando comporado aos estudos de sistemas ecológicos equivalentes, apresentados na bibliografia. 


\section{RECOMENDAÇÕES}

1) Maior precisão do método pode ser obtida com a identificação dos microrganismos existentes.

2) Redução no intervalo de tempo entre as amostragens.

3) Aumento do periodo total do experimento.

4) Aumento do periodo de observação após a aplicação dos tóxicos.

5) Utilização de técnicas modernas no estudo da diversidade biológica.

6) Aplicação de modelos matemáticos de dinâmica populacional e outros modelos ecológicos. 


\section{REFERÊNCIAS}

1. Andrei. Compendêndio de defensivos agrícolas. São Paulo: Organização Andrei Editora; 1985.

2. Beyers RJ. Ecological monographs. The metabolism of twelve aquatic laboratory microecosystems. Durham: Duke University Press; 1963, p. 281306.

3. Beyers RJ. The microcosm approach to ecosystem biology. Am. Biol. Teacher 1964; 26(7): 491-498.

4. Beyers RJ, Odum HT. Ecological microcosms. New York: Springer Verlag; 1993.

5. Berk SG, Gunderson JH. Wastewater organisms a colour atlas. Boca Ratom: Lewis publication; 1993.

6. Botkin DB, Wilson MV. Models of simple microcosms: emergent properties and the effect of complexity on stability. Am naturalist 1990; 135(3):414434.

7. Boudou A, Ribeyre F. Aquatic ecolotoxicology: fundamentals concepts and methodologies. Boca Ratom: CRC Press; 1988. 
8. Branco SM. Hidrobiologia aplicada à engenharia sanitária. $3^{\mathrm{a}}$ ed. São Paulo: CETESB; 1986.

9. Cairns JJr. Microcosm pollution monitoring. Trace Subst Environ Health, $1974 ; 8: 223-228$.

10. Cairns JJr, Pratt JR. The scientific basis of bioassays. In: . M. Munawar, G. Dixon, C. I. Mayfield, T. Reynoldson \& H. Sadar editors. Environmental bioassay techniques and their application. Dordrecht: Kluer Academic Publishers; 1989, p.5-20.

11. Cairns JJr, Niederlehner BR. Ecological toxicity testing. Boca Ratom; CRC Press; 1995.

12. Calow P. General principals and overview in handbook of ecotoxicology. Oxford: Blackwell Science; 1995.

13. Curtis TP, Craine NG. The comparison of the diversity of activated sludge plants. Water Sci Tech 1998; 37:71-78.

14. Curtis TP, Head I. Random bacterial community assembly in activated sludge: a new perspective on engineered biological systems. Newcastle: University of Newcastle; 1999.

15. Fiskesjö G. Allium test in wastewater monitoring. Environ Toxicol Water Qual 1993; 8:291-98. 
16. Hutchinson GE. A treatise on limnology I: Geography, physics, and chemistry. New York; Wiley; 1959.

17. Kelly JR, Harwell MA. Indicators of ecosystem response and recovery. In: Levan S. A. Harwell, M.A, Kelly J. R. e Kimball D.K. editors. Ecotoxicology: problems and approaches. New York: Springer Verlag; 1989, p. 9-35.

17. May RM. Stability and complexity in model ecosystems. New Jersey: Princeton University Press; 1975.

18. Mayfield CI. Microbial systems in handbook of ecotoxicology. Oxford: Blackwell Science, 1995. p 9-27.

19. McKinney RE. Microbiology for sanitary engineers. New York: McGrawHill; 1962.

20. Metcalf RL, Sancha GK, Kapoor IP. Environ Sci Technol 1971; 5 (8):709713.

21. MACARTHUR RH, WILSON EO. The theory of biogeography island. New Jersey: Princeton Universy Press; 1967.

22. Muyzer G, Smalla K. Application of denaturing gradient gel electrophoresis (DGGE) and temperature gradient gel electrophoresis (TGGE) in microbial ecology. Antonie van Leeuwenhoek 1998; 73:127-141. 
23. Muyzer G. DGGE/TGGE a method for identifying genes from natural ecosystems. Current Opinion Microbiol 1999; 2:317-322.

24. Odum EP. Ecologia. Rio de Janeiro: Editora Guanabara; 1983.

25. Pratt JR, Niederlehner BR, Bowers N, Cairns JJr. Prediction of permissible concentrations of copper from microcosm toxicity tests. Toxicity Assessment: An Intern Quaterly 1987; 2:417-436.

26. Rand GM, Petrocelli JR. Fundamental of aquatic toxicology. Washington, D. C.: MacGraw Hill; 1985.

26. Ribeiro IA. Teste com raízes de cebola para avaliação de toxicidade de efluentes industriais. São Paulo, 1997. [Dissertação de Mestrado Faculdade de Saúde Pública da U.S.P.].

28. Rodrigues RS. Avaliação do nível de resíduos de atrazine e simazine em água, em áreas de cultivo intensivo de milho no Brasil. São Paulo; 2001. [Tese de Doutorado -Instituto de Biociências da U.S.P.].

29. Sáfadi RS. Avaliação da toxicidade de fluidos de perfuração de poços de petróleo e gás para os organismos marinhos Mysidopsis juniae (Crustacea, Mysidacea) e Lytechinus variegatus (Echinodermata, Echinoidea). São Paulo; 2001 [Tese de Doutorado - Instituto de Biociências da U.S.P.]. 
30. Salt GW. A comment on the use of the term emergent properties. Am Nat 1979; 113:145-148.

31. Taub FB. Demonstration of pollution effects in aquatic microcosms. Int $\mathbf{J}$ Environ Stud 1976; 10:23-33.

32. Wilson MV, Botkin D. Models of simple microcosms: emergent properties and effect of complexity on stability. Am Naturalist 1990; 135:414-34.

33. Wimpenny JWT. Handbook of laboratory model systems for microbial ecosystems. Florida: CRC Press; 1988. 


\section{ANEXO 1}

\section{PLANILHAS}

- EXPERIMENTO I

- EXPERIMENTO II

- EXPERIMENTO III 
Experimento I

\begin{tabular}{|c|c|c|c|c|c|c|c|c|c|c|}
\hline \multicolumn{11}{|c|}{ ilamentos } \\
\hline Amostra & lia 0 & dia 1 & dia 2 & 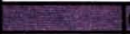 & dia 4 & dia 5 & dia 6 & dia 7 & dia 8 & dia 9 \\
\hline 1 & 0 & 이 & 1 & 1 & 0 & 7 & 47 & 33 & 29 & 46 \\
\hline 2 & 0 & 0 & 2 & 3 & 0 & 7 & 44 & 29 & 34 & 46 \\
\hline 3 & 0 & 0 & 1 & 4 & 1 & 13 & 42 & 27 & 30 & 78 \\
\hline 4 & 0 & 0 & 1 & 4 & 1 & 14 & 48 & 30 & 29 & 46 \\
\hline 5 & 0 & 0 & 1 & 2 & 1 & 8 & 45 & 30 & 30 & 46 \\
\hline 6 & 0 & 0 & 2 & 1 & 1 & 11 & 45 & 32 & 30 & 55 \\
\hline 7 & 0 & 0 & 3 & 4 & 1 & 10 & 49 & 30 & 29 & 66 \\
\hline 8 & 0 & 0 & 2 & 2 & 1 & 9 & 50 & 31 & 28 & 54 \\
\hline 9 & 0 & 0 & 1 & 2 & 0 & 8 & 44 & 30 & 27 & 47 \\
\hline 10 & 0 & 0 & 1 & 2 & 0 & 10 & 45 & 31 & 30 & 47 \\
\hline 11 & 0 & & 1 & 2 & 0 & 11 & 46 & 30 & 33 & 36 \\
\hline 12 & 0 & 0 & 1 & 2 & 0 & 12 & 47 & 30 & 33 & 45 \\
\hline Média & $x$ & 0,00 & 1,42 & 2,42 & 0,50 & 10,00 & 46,00 & 30,25 & 30,17 & 51,00 \\
\hline Temp ${ }^{\circ} \mathrm{C}$ & & 22,0 & 22,0 & 23,0 & 25,0 & 25,0 & 22,0 & 23,0 & 23,0 & 24,0 \\
\hline $\mathrm{pH}$ & & 7,2 & 7,2 & 7,25 & 7,83 & \begin{tabular}{ll|}
7,96 \\
\end{tabular} & 7,92 & 7,77 & 7,9 & 7,88 \\
\hline
\end{tabular}
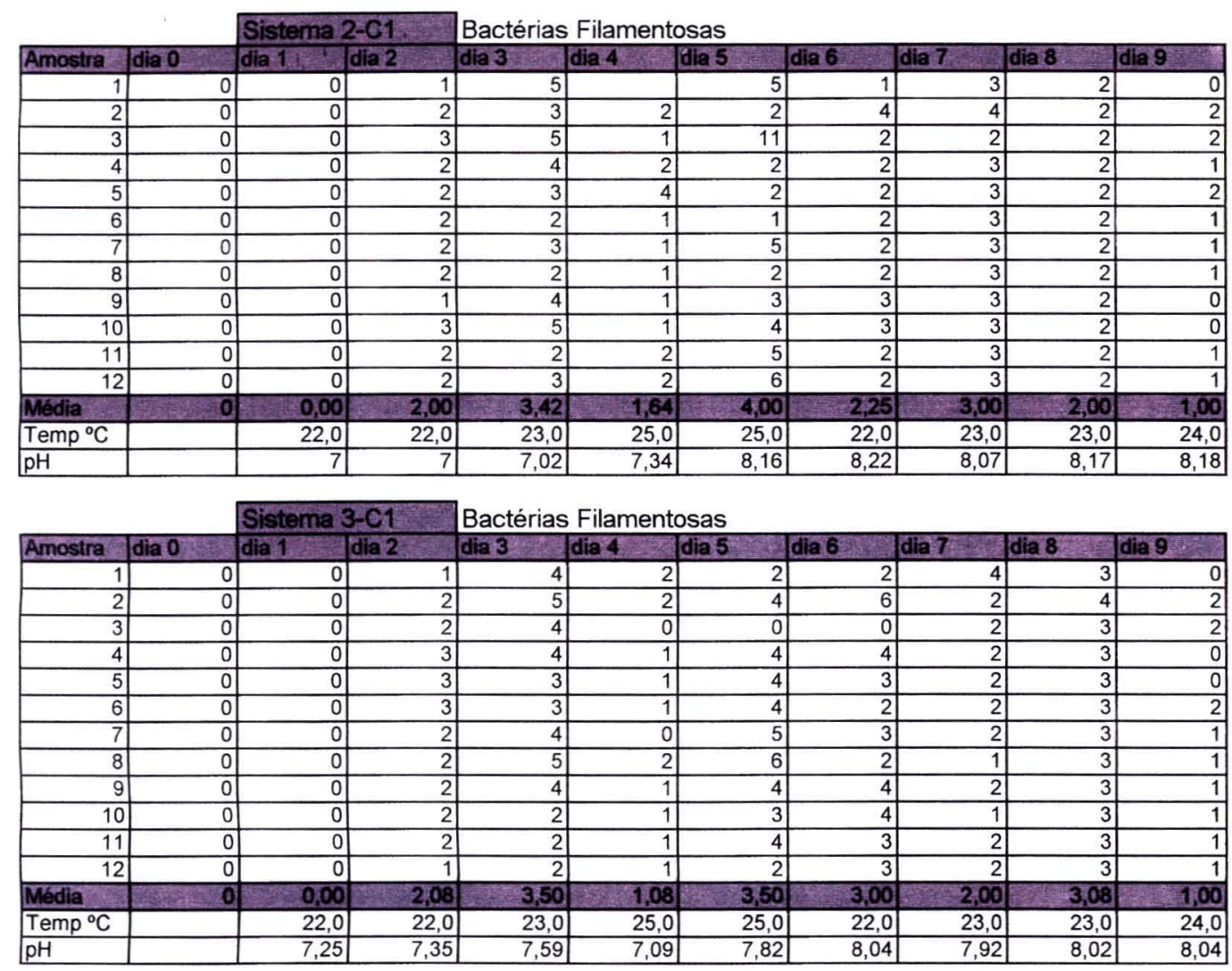

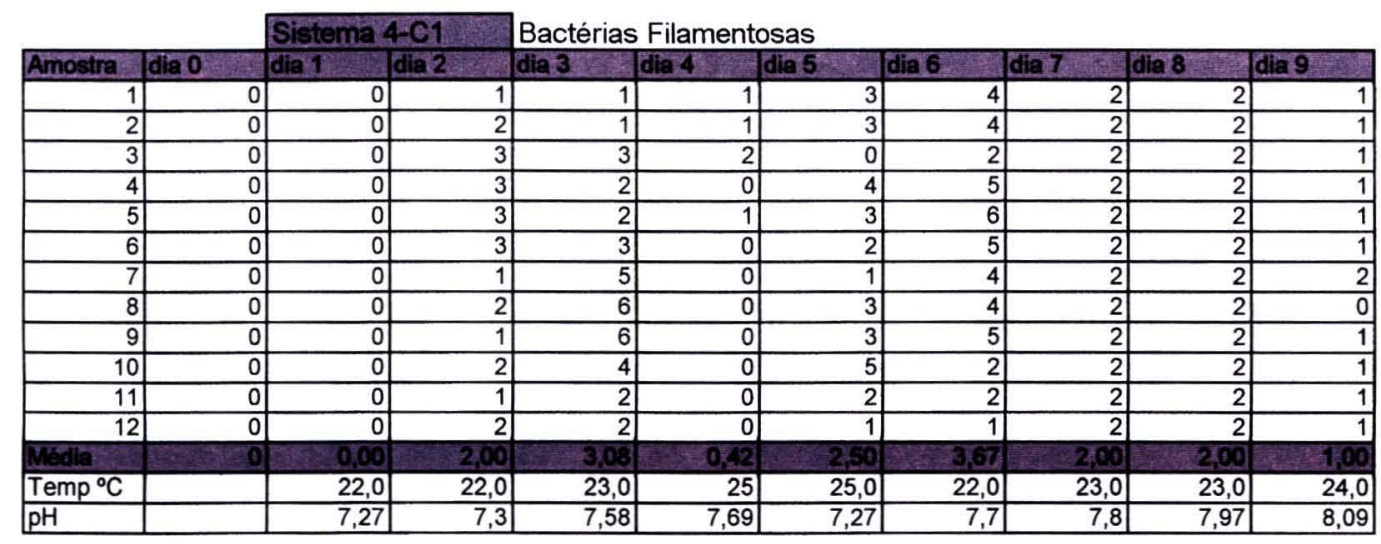




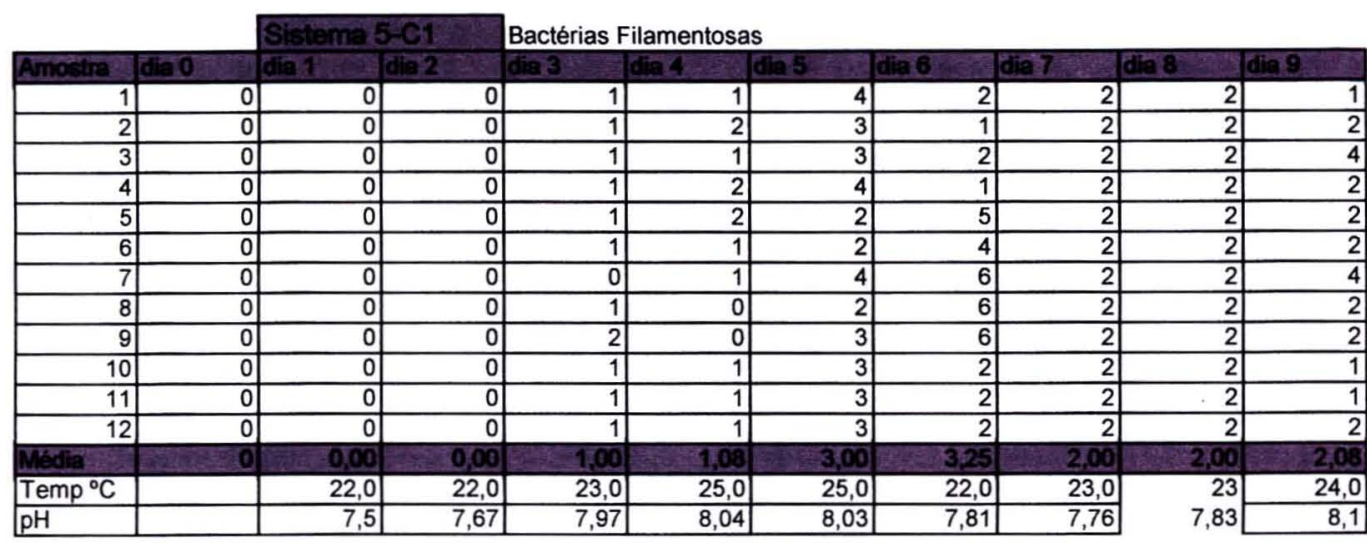

Quantidade de microrganismos/substrato DBO de $350 \mathrm{mg} / \mathrm{N}$ aeraça contínual $1,56 \mathrm{ppm}$ de Cobre no $4^{\circ}$ di

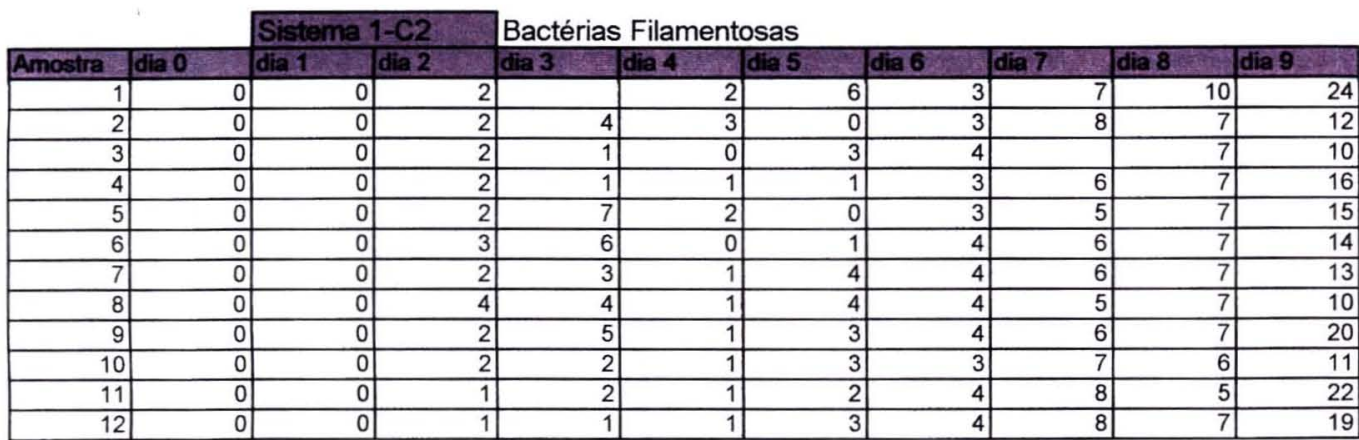

\begin{tabular}{|l|r|r|r|r|r|r|r|r|r|r|}
\hline Média & 0 & 0,00 & 2,08 & 3,27 & 1,17 & 2,50 & 3,58 & 6,55 & 7,00 & 15,50 \\
\hline Temp ${ }^{\circ} \mathrm{C}$ & & 22,0 & 22,0 & 23,0 & 25,0 & 25 & 22,0 & 23,0 & 23,0 & 24,0 \\
\hline $\mathrm{pH}$ & & 7 & 7 & 7 & 7,17 & 7,14 & 7,43 & 7,44 & 7,6 & 7,52 \\
\hline
\end{tabular}

\begin{tabular}{|c|c|c|c|c|c|c|c|c|c|c|}
\hline & & Sistema? & $2 c-2$ & Bactérias & Filamentc & osas & & & & \\
\hline Amostra & dia 0 & dia 1 & dia 2 & dia 3 & dia 4 & dia 5 & Tia 6 & dia 7 & dia 8 & dia 9 \\
\hline 1 & 0 & 0 & 1 & 2 & 2 & 2 & 2 & 3 & 2 & 1 \\
\hline 2 & 0 & 0 & 1 & & 2 & 1 & 2 & 3 & 3 & 3 \\
\hline 3 & 0 & 0 & 1 & 2 & 0 & 2 & 1 & 3 & 2 & 4 \\
\hline 4 & 0 & 0 & 1 & 2 & 1 & 2 & 2 & 3 & 2 & 2 \\
\hline 5 & 0 & 0 & 1 & 2 & 0 & 0 & 1 & 3 & 2 & 2 \\
\hline 6 & 0 & 0 & 1 & 2 & 2 & 0 & 2 & 3 & 2 & 2 \\
\hline 7 & 0 & 0 & 2 & 2 & 2 & 0 & 3 & 3 & 2 & 2 \\
\hline 8 & 0 & 0 & 1 & 2 & 2 & 2 & 3 & 3 & 2 & 2 \\
\hline 9 & 0 & 0 & 2 & 3 & 1 & 2 & 2 & 3 & 2 & 2 \\
\hline 10 & 0 & 0 & 1 & 2 & 2 & 2 & 2 & 3 & 2 & 2 \\
\hline 11 & 0 & 0 & 2 & 2 & 2 & 1 & 3 & 3 & 2 & 2 \\
\hline 12 & 0 & 0 & 2 & 2 & 2 & 1 & 3 & 3 & 2 & 2 \\
\hline \begin{tabular}{|l|} 
Média \\
\end{tabular} & 0 & 0,00 & 1,33 & 2,09 & 1,50 & 1,25 & 2,17 & 3,00 & 2,08 & 2,17 \\
\hline $\operatorname{Temp}^{\circ} \mathrm{C}$ & & 22,0 & 22,0 & 23,0 & 25,0 & 25 & 22,0 & 23,0 & 23,0 & 24,0 \\
\hline $\mathrm{pH}$ & & 7,1 & 7,15 & 7,59 & 7,41 & 7,87 & 7,7 & 7,92 & 8,15 & 8,18 \\
\hline
\end{tabular}

\begin{tabular}{|c|c|c|c|c|c|c|c|c|c|c|c|}
\hline Amostra & dia 0 & & dia 1 & dia 2 & dia 3 & dia 4 & dia 5 & dia 6 & dia 7 & dia 8 & dia 9 \\
\hline 1 & & 0 & 0 & 1 & 1 & 2 & 0 & 2 & 1 & 3 & 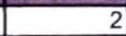 \\
\hline 2 & & 0 & 0 & 1 & 2 & 2 & 1 & 2 & 2 & 3 & 2 \\
\hline 3 & & 0 & 0 & 1 & 3 & 2 & 1 & 1 & 2 & 3 & 2 \\
\hline 4 & & 0 & 0 & 1 & 2 & 1 & 1 & 1 & 2 & 3 & 0 \\
\hline 5 & & 0 & 0 & 2 & 2 & 1 & 2 & 1 & 2 & 3 & 2 \\
\hline 6 & & 0 & 0 & 2 & 1 & 2 & 0 & 1 & 2 & 2 & 2 \\
\hline 7 & & 0 & 0 & 1 & 1 & 1 & 2 & 1 & 2 & 2 & 1 \\
\hline 8 & & 0 & 0 & 1 & 2 & 2 & 2 & 1 & 2 & 2 & 2 \\
\hline 9 & & 0 & 0 & 1 & 1 & 1 & 1 & 1 & 2 & 1 & 1 \\
\hline 10 & & 0 & 0 & 1 & 2 & 2 & 1 & 1 & 2 & 2 & 2 \\
\hline 11 & & 0 & 0 & 1 & 2 & 1 & 1 & 1 & 3 & 0 & 0 \\
\hline 12 & & 0 & 0 & 1 & 1 & 1 & 1) & 1 & 2 & 0 & 0 \\
\hline Média & & 0 & 0,00 & 1,17 & 1,67 & 1,50 & 1,08 & 1,17 & 2,00 & 2,00 & 2,00 \\
\hline Temp ${ }^{\circ} \mathrm{C}$ & & & 22,0 & 22,0 & 23,0 & 25,0 & 25 & 22,0 & 23,0 & 23,0 & 24,0 \\
\hline $\mathrm{pH}$ & & & 7,01 & 7,01 & 7,03 & 7,3 & 7,37 & 7,7 & 7,58 & 7,71 & 7,78 \\
\hline
\end{tabular}




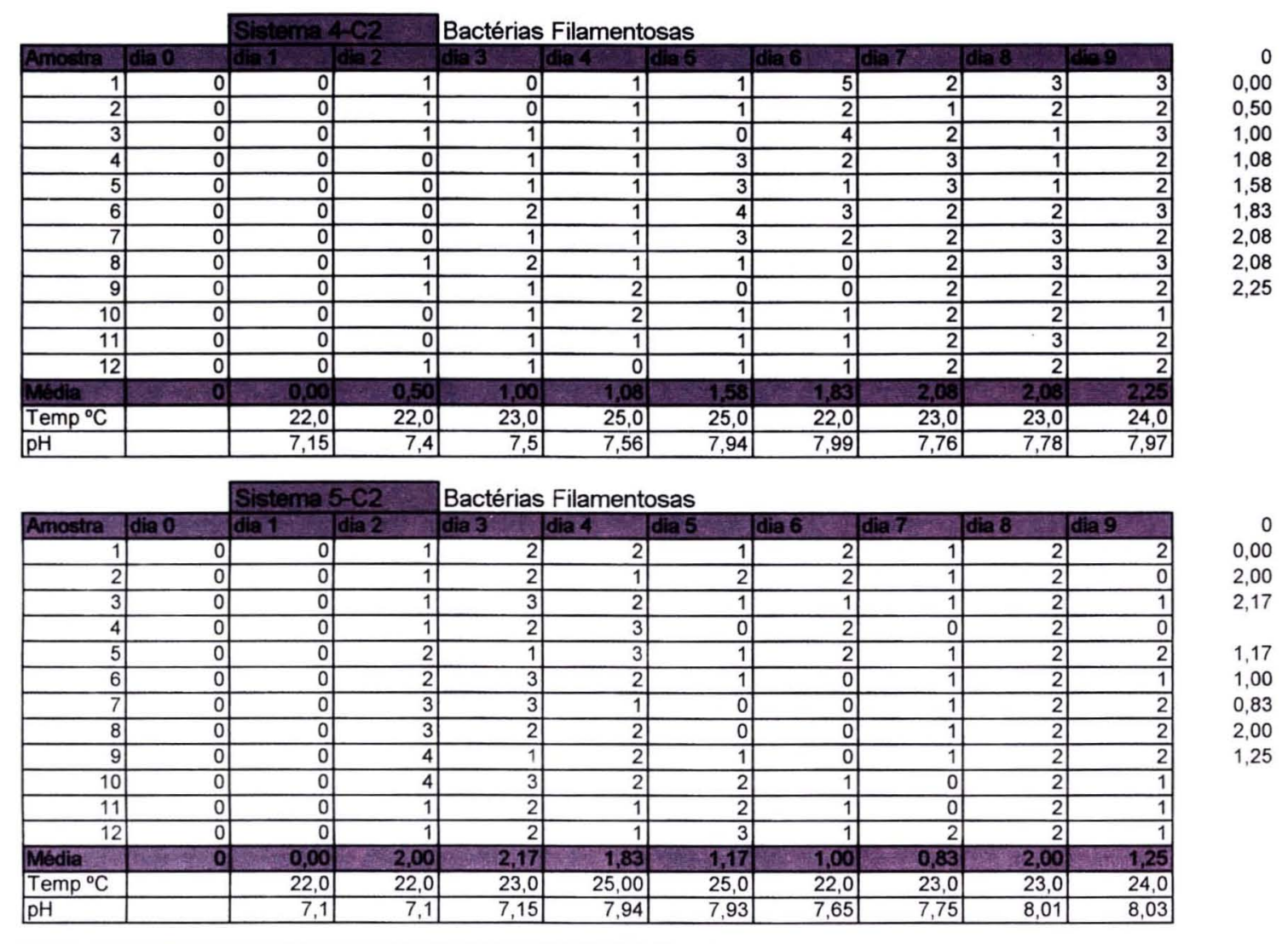

Quantidade de microrganismos/substrato DBO de $350 \mathrm{mg} / /$ aeraçắ continua/3,12 ppm de Cobre no $4^{\circ}$ dia

\begin{tabular}{|c|c|c|c|c|c|c|c|c|c|c|c|c|c|}
\hline & & Sistema & $1-C 3$ & & Bactérias & Filamento & osas & & & & & & \\
\hline Amostra & $\operatorname{dia} 0$ & dia 1 & dia 2 & 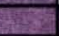 & dia 3 & dia 4 & dia 5 & & dia 6 & dia 7 & |dia 8 & dia 9 & 0 \\
\hline $\begin{array}{r}1 \\
\end{array}$ & 1 & 0 & & 1 & & 2 & & 2 & 2 & 2 & 4 & 2 & 0,75 \\
\hline 2 & 2 & 3 & & 0 & 0 & 3 & & 5 & 2 & 2 & 3 & 2 & 0,33 \\
\hline 3 & 3 & 0 & & 0 & 0 & 2 & & 4 & 1 & 2 & 0 & 0 & 0,00 \\
\hline 4 & 4 & 0 & & 0 & 0 & 4 & & 1 & 1 & 1 & 0 & 3 & 2,08 \\
\hline 5 & 5 & 4 & & 0 & 0 & 2 & & 4 & 2 & 2 & 0 & 5 & 3,00 \\
\hline 6 & 6 & 0 & & 0 & 0 & 1 & & 2 & 2 & 0 & 0 & 3 & 1,25 \\
\hline & 7 & 0 & & 1 & 0 & 1 & & 3 & 1 & 1 & 1 & 4 & 0,83 \\
\hline 8 & 8 & 0 & & 0 & 0 & 2 & & 3 & 1 & 0 & 0 & 2 & 1,00 \\
\hline 9 & 9 & 0 & & 0 & 0 & 3 & & 3 & 1 & 0 & 2 & 2 & 2,08 \\
\hline 10 & & 0 & & 1 & 0 & 2 & & 3 & 1 & 0 & 0 & 1 & \\
\hline 11 & & 1 & & 1 & 0 & 1 & & 3 & 1 & 0 & 1 & 1 & \\
\hline 12 & & 1 & & 0 & 0 & 2 & & 3 & 0 & 0 & 1 & 0 & \\
\hline Média & & 0,75 & E & 0,33 & 0,00 & 2,08 & +5 & 3,00 & 1,25 & 0,83 & 1,00 & 2,08 & \\
\hline Temp ${ }^{\circ} \mathrm{C}$ & & 20,0 & & 21,0 & 23,0 & 23,0 & & 22,0 & 22,0 & 23,0 & 22,0 & 23,0 & \\
\hline $\mathrm{pH}$ & & 7,3 & & 7,58 & 7,75 & 7,64 & & 7,69 & 7,72 & 7,92 & 7,99 & 7,87 & \\
\hline & & Sistema & $2-c 3$ & & Bactérias & Filamento & osas & & & & & & \\
\hline Amostra & dia 0 & dia 1 & $\longdiv { \text { dia } 2 }$ & 7 & \begin{tabular}{|c|}
$d i a$ \\
3
\end{tabular} & $\begin{array}{ll}\text { dia } 4 \\
\end{array}$ & dia 5 & 1 & dia 6 & dia 7 & dia 8 & dia 9 & 0 \\
\hline 1 & & 5 & & 0 & 0 & 2 & & 1 & 1 & 2 & 2 & 2 & 1,17 \\
\hline 2 & & 1 & & 1 & 0 & 1 & & 0 & 1 & 1 & 3 & 1 & 0,17 \\
\hline 3 & 3 & 0 & & 0 & 0 & 2 & & 1 & 0 & 1 & 4 & 3 & 0,00 \\
\hline 4 & 4 & 0 & & 0 & 0 & 3 & & 1 & 2 & 1 & 2 & 1 & 2,92 \\
\hline 5 & 5 & 0 & & 0 & 0 & 2 & & 0 & 3 & 1 & 5 & 1 & 0,25 \\
\hline 6 & 匀 & 1 & & 0 & 0 & 3 & & 0 & 3 & 1 & 6 & 1 & 1,58 \\
\hline 7 & & 5 & & 0 & 0 & 3 & & 0 & 4 & 0 & 2 & 1 & 0,75 \\
\hline 8 & & 0 & & 1 & 0 & 4 & & 0 & 2 & 1 & 1 & 1 & 2,67 \\
\hline 9 & & 0 & & 0 & 0 & 6 & & 0 & 1 & 0 & 1 & 2 & 1,50 \\
\hline 10 & & 1 & & 0 & 0 & 5 & & 0 & 2 & 0 & 2 & 1 & \\
\hline 11 & & 1 & & 0 & 0 & 2 & & 0 & 0 & 1 & 2 & 2 & \\
\hline 12 & & 0 & & 0 & 0 & 2 & & 0 & 0 & 0 & 2 & 2 & \\
\hline Media & & 1,17 & & 0,17 & 0,00 & 2,92 & & 0,25 & 1,58 & 0.75 & 2,67 & 1,50 & \\
\hline $\operatorname{Temp}^{\circ} \mathrm{C}$ & & 20,0 & & 21,0 & 23,0 & 23,0 & & 22,0 & 22,0 & 23,0 & 22,0 & 23,0 & \\
\hline $\mathrm{pH}$ & & 7,07 & & 7,54 & 7,39 & 7,05 & & 7,16 & 7,3 & 7,41 & 7,4 & 7,87 & \\
\hline
\end{tabular}




\begin{tabular}{|c|c|c|c|c|c|c|c|c|c|c|}
\hline \multirow{3}{*}{\multicolumn{2}{|c|}{\begin{tabular}{|l|l|l|} 
Amostre & dia 0 \\
1
\end{tabular}}} & \multicolumn{2}{|c|}{ Sistema 3-C3 } & \multicolumn{7}{|c|}{ Bactérias Filamentosas } \\
\hline & & & dia 2 & dia 3 & dia 4 & Tia 5 & dita 6 & dia 7 & dia 8 & 29 \\
\hline & & 0 & 0 & 0 & 1 & 0 & 0 & 0 & 2 & 0 \\
\hline 2 & & 0 & 1 & 0 & 2 & 1 & 0 & 0 & 2 & 3 \\
\hline 3 & & 1 & 0 & 0 & 3 & 2 & 0 & 0 & 0 & 1 \\
\hline 4 & & 1 & 0 & 0 & 2 & 2 & 0 & 0 & 1 & 3 \\
\hline 5 & & 0 & 1 & 0 & 5 & 1 & 0 & 0 & 2 & 2 \\
\hline 6 & & 1 & 1 & 2 & 4 & 2 & 0 & 0 & 0 & 1 \\
\hline 7 & & 0 & 0 & 2 & 3 & 2 & 2 & 0 & 1 & 1 \\
\hline 8 & & 4 & 0 & 0 & 2 & 3 & 0 & 2 & 1 & 1 \\
\hline 9 & & 2 & 0 & 0 & 1 & 1 & 0 & 1 & 1 & 1 \\
\hline 10 & & 2 & 0 & 0 & 1 & 2 & 0 & 1 & 0 & 1 \\
\hline 11 & & 2 & 1 & 0 & 1 & 1 & 1 & 1 & 0 & 2 \\
\hline 12 & & 2 & 0 & 0 & 0 & 1 & 2 & 1 & 0 & 2 \\
\hline Média & & 1,25 & 0,33 & 0,33 & 2,08 & 1,50 & 0,42 & 0,50 & 0,83 & 1,50 \\
\hline $\operatorname{Temp}^{\circ} \mathrm{C}$ & & 20,0 & 21,0 & 23,0 & 23,0 & 22,0 & 22,0 & 23,0 & \begin{tabular}{|l|}
22,0 \\
\end{tabular} & 23,0 \\
\hline $\mathrm{pH}$ & & \begin{tabular}{ll|}
7,03 \\
\end{tabular} & 6,41 & 7,45 & 7,77 & 7,94 & $\begin{array}{l}7,89 \\
\end{array}$ & 8,11 & \begin{tabular}{l|l|}
8,08 \\
\end{tabular} & 8,04 \\
\hline
\end{tabular}

Sistema 4-C3 Bactérias Filamentosas

\begin{tabular}{|c|c|c|c|c|c|c|c|c|c|c|}
\hline Amostra & dia 0 & dia 1 & dia 2 & dia 3. & dia 4 & dia 5 & dia 6 & dia 7 & dia 8 & dia 9 \\
\hline 1 & & 0 & 0 & $\begin{array}{r}0 \\
\end{array}$ & 2 & 0 & 0 & 0 & 0 & 0 \\
\hline 2 & & 0 & 0 & 0 & 2 & 0 & 0 & 0 & 1 & 2 \\
\hline 3 & & 0 & 0 & 0 & 4 & 1 & 0 & 0 & 1 & 1 \\
\hline 4 & & 0 & 0 & 0 & 2 & 0 & 0 & 0 & 0 & 1 \\
\hline 5 & & 0 & 0 & 0 & 5 & 1 & 1 & 1 & 0 & 1 \\
\hline 6 & & 0 & 0 & 1 & 3 & 1 & 1 & 1 & 1 & 1 \\
\hline 7 & & 1 & 1 & 0 & 1 & 0 & 1 & 1 & 0 & 1 \\
\hline 8 & & 2 & 1 & 0 & 1 & 1 & 2 & 1 & 0 & 1 \\
\hline 9 & & 1 & 0 & 0 & 1 & 1 & 0 & 0 & 0 & 1 \\
\hline 10 & & 1 & 0 & 0 & 2 & 0 & 0 & 0 & 0 & 1 \\
\hline 11 & & 0 & 0 & 0 & 2 & 0 & 0 & 0 & 0 & 1 \\
\hline 12 & & 2 & 0 & 0 & 2 & 0 & 0 & 0 & 0 & 1 \\
\hline Média & & 0,58 & 0,17 & 0,08 & 2.25 & 0.42 & 0,42 & 0,33 & 0,25 & 1,00 \\
\hline Temp $^{\circ} \mathrm{C}$ & & 20,0 & 21,0 & 23,0 & 23,0 & 22,0 & 22,0 & 23,0 & 22,0 & 23,0 \\
\hline $\mathrm{pH}$ & & 7,28 & 7,12 & 7,42 & 7,69 & 7,84 & 7,9 & 7,94 & 7,96 & 7,87 \\
\hline
\end{tabular}

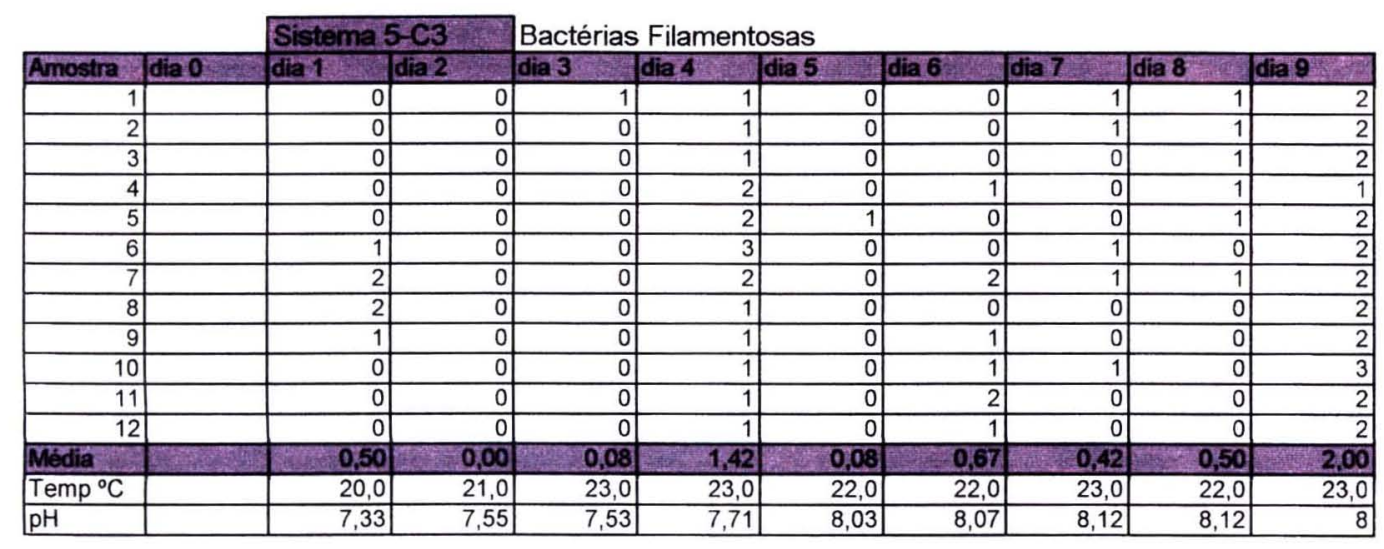


Experimento I

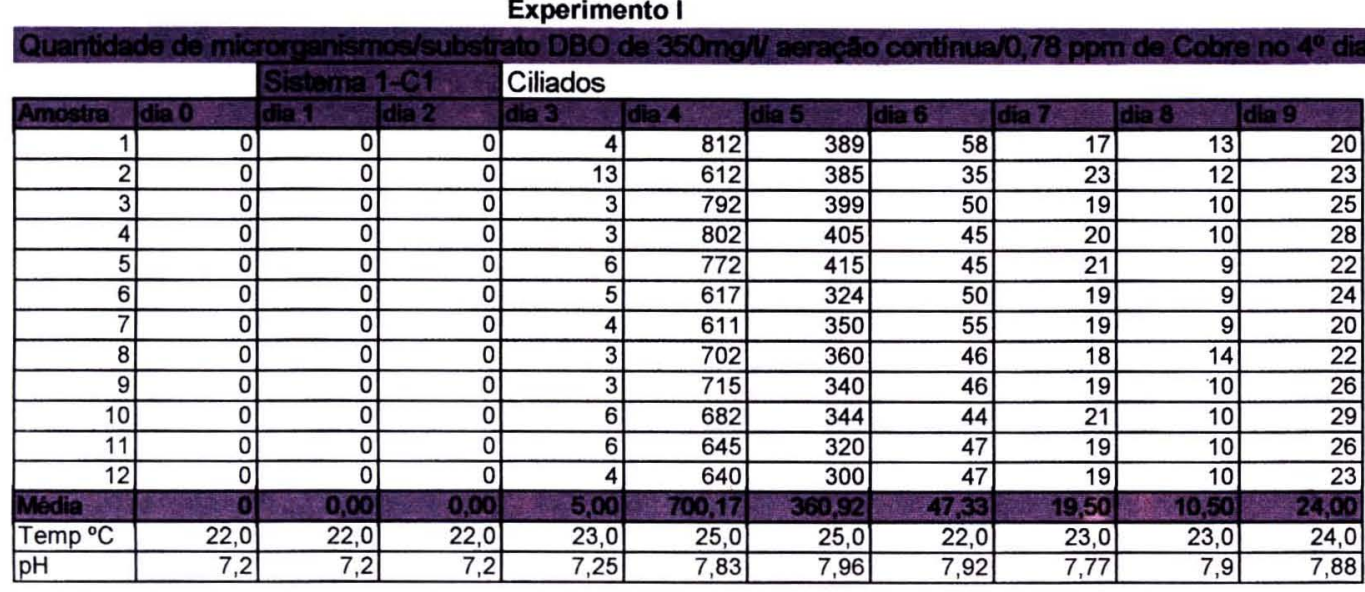

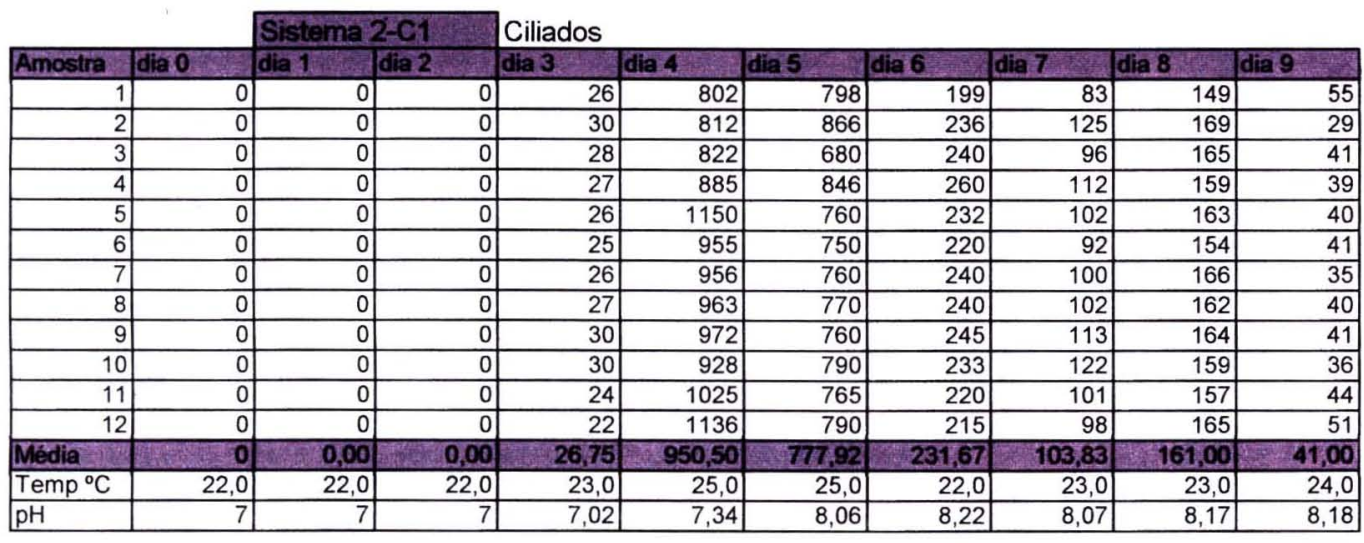

\begin{tabular}{|c|c|c|c|c|c|c|c|c|c|c|}
\hline & & Sistema : & $3-\mathrm{Cl}$ & Ciliados & & & & & & \\
\hline Amostra & dia 0 & dia 1 & dia 2 & dia 3 & dia 4 & dia 5 & dia 6 & dia 7. & dia 8 & dia 9 \\
\hline $\begin{array}{r}1 \\
\end{array}$ & 0 & 0 & 0 & 3 & 102 & 254 & 114 & 84 & 182 & 155 \\
\hline 2 & 0 & 0 & 0 & 5 & 62 & 285 & 225 & 155 & 189 & 212 \\
\hline 3 & 0 & 0 & 0 & 2 & 82 & 234 & 240 & 164 & 155 & 201 \\
\hline 4 & 0 & 0 & 0 & 3 & 55 & 234 & 200 & 132 & 165 & 185 \\
\hline 5 & 0 & 0 & 0 & 2 & 62 & 193 & 195 & 121 & 184 & 201 \\
\hline 6 & 0 & 0 & 0 & 5 & 44 & 284 & 195 & 136 & 169 & 223 \\
\hline 7 & 0 & 0 & 0 & 3 & 63 & 261 & 280 & 133 & 194 & 192 \\
\hline 8 & 0 & 0 & 0 & 2 & 77 & 250 & 190 & 135 & 196 & 185 \\
\hline 9 & 0 & 0 & 0 & 3 & 66 & 256 & 160 & 140 & 195 & 171 \\
\hline 10 & 0 & 0 & 0 & 3 & 69 & 246 & 185 & 135 & 199 & 165 \\
\hline 11 & 0 & 0 & 0 & 3 & 60 & 255 & 180 & 145 & 187 & 212 \\
\hline 12 & 0 & 0 & 0 & 3 & 77 & 252 & 170 & 130 & 198 & 192 \\
\hline Média & 0 & 0,00 & 0,00 & 3,08 & 68,25 & 250,33 & 194,50 & 134,17 & 184,42 & 191,17 \\
\hline Temp ${ }^{\circ} \mathrm{C}$ & 22,0 & 22,0 & 23,0 & 23,0 & 25,0 & 25,0 & 22,0 & 23,0 & 23,0 & 24,0 \\
\hline $\mathrm{pH}$ & 7,1 & 7,25 & 7,35 & 7,59 & 7,09 & 7,82 & 8,04 & 7,92 & 8,02 & 8,04 \\
\hline
\end{tabular}

\begin{tabular}{|c|c|c|c|c|c|c|c|c|c|c|}
\hline & & Sistema & H-C1 & Ciliados & & & & & & \\
\hline Amostra & dia 0 & dia 1 & dia 2 & dia 3 & dia 4 & dia 5 & dia 6 & dia 7 & dia 8 & dia 9 \\
\hline 1 & 0 & 0 & 0 & 2 & 225 & 343 & 488 & 210 & 84 & 7 \\
\hline 2 & 0 & 0 & 0 & 2 & 256 & 306 & 640 & 180 & 85 & 3 \\
\hline 3 & 0 & 0 & 0 & 5 & 232 & 318 & 580 & 200 & 90 & 5 \\
\hline 4 & 0 & 0 & 0 & 6 & 212 & 365 & 560 & 190 & 95 & 5 \\
\hline 5 & 0 & 0 & 0 & 3 & 255 & 352 & 550 & 195 & 85 & 6 \\
\hline 6 & 0 & 0 & 0 & 5 & 265 & 360 & 540 & 200 & 86 & 4 \\
\hline 7 & 0 & 0 & 0 & 8 & 221 & 320 & 560 & 210 & 92 & 5 \\
\hline 8 & 0 & 0 & 0 & 9 & 322 & 335 & 570 & 205 & 95 & 3 \\
\hline 9 & 0 & 0 & 0 & 6 & 312 & 320 & 560 & 200 & 89 & 6 \\
\hline 10 & 0 & 0 & 0 & 3 & 305 & 310 & 580 & 190 & 86 & 5 \\
\hline 11 & 0 & 0 & 0 & 5 & 290 & 290 & 580 & 196 & 80 & 7 \\
\hline 12 & 0 & 0 & 0 & 6 & 225 & 310 & 590 & 180 & 72 & 4 \\
\hline Media & 0 & 0,00 & 0,00 & 5,00 & 260,00 & 327,42 & 566,50 & 196,33 & 86,58 & 5,00 \\
\hline $\operatorname{Temp}^{\circ} \mathrm{C}$ & 22,0 & 22,0 & 22,0 & 23,0 & 25,0 & 25,0 & 22,0 & 23,0 & 23,0 & 24,0 \\
\hline $\mathrm{pH}$ & 7,27 & 7,27 & 7,3 & 7,58 & 7,69 & 7,27 & 7,7 & 7,8 & 7,97 & 8,09 \\
\hline
\end{tabular}




\begin{tabular}{|c|c|c|c|c|c|c|c|c|c|c|}
\hline & & istema & 5-C1 & Ciliados & & & & & & \\
\hline istra & 10 & dian 1 & dia 2 & dia 3 & 134 & 35 & & & & \\
\hline 1 & 0 & 0 & $\overline{0}$ & 1 & 422 & 720 & 598 & 300 & 300 & 0 \\
\hline 2 & 0 & 0 & 0 & 1 & 442 & 900 & 595 & 360 & 320 & 2 \\
\hline 3 & 0 & 0 & 0 & 1 & 536 & 945 & 550 & 350 & 330 & 2 \\
\hline 4 & 0 & 0 & 0 & 1 & 612 & 866 & 590 & 350 & 345 & 0 \\
\hline 5 & 0 & 0 & 0 & 5 & 448 & 900 & 680 & 320 & 400 & 0 \\
\hline 6 & 0 & 0 & 0 & 6 & 507 & 950 & 550 & 320 & 410 & 2 \\
\hline 7 & 0 & 0 & 0 & 5 & 542 & 900 & 540 & 320 & 350 & 1 \\
\hline 8 & 0 & 0 & 0 & 6 & 467 & 750 & 590 & 325 & 360 & 1 \\
\hline 9 & 0 & 0 & 0 & 5 & 462 & 780 & 600 & 330 & 346 & 1 \\
\hline 10 & 0 & 0 & 0 & 6 & 446 & 860 & 600 & 325 & 349 & 1 \\
\hline 11 & 0 & 0 & 0 & 5 & 432 & 870 & 650 & 336 & 349 & 1 \\
\hline 12 & 0 & 0 & 0 & 7 & 469 & 890 & 640 & 335 & 350 & 1 \\
\hline Média & 0 & 0,00 & 0,00 & 4,08 & 482,08 & 860,92 & 598,58 & 330,92 & 350,75 & 1,00 \\
\hline $\operatorname{Temp}^{\circ} \mathrm{C}$ & 22,0 & 22,0 & 22,0 & 23,0 & 25,0 & 25,0 & 22,0 & 23,0 & 23,0 & 24,0 \\
\hline $\mathrm{pH}$ & 7 & 7,5 & 7,67 & 7,97 & 8,04 & 8,03 & 7,81 & 7,76 & 7,83 & 8,1 \\
\hline
\end{tabular}

\begin{tabular}{|c|c|c|c|c|c|c|c|c|c|c|}
\hline & , & Sistema & $1-C 2$ & Ciliados & & & & & & \\
\hline Amostra & dia 0 & dia 1 & dia 2 & dia 3 , & dia 4 & dia 5 & dia 6 & dia 7 & dia 8 & dia 9 \\
\hline 1 & 0 & 0 & 0 & 9 & 344 & 15 & 85 & 351 & 355 & 5 \\
\hline 2 & 0 & 0 & 0 & 8 & 324 & 26 & 98 & 432 & 372 & 8 \\
\hline 3 & 0 & 0 & 0 & 12 & 392 & 22 & 122 & 359 & 366 & 15 \\
\hline 4 & 0 & 0 & 0 & 12 & 312 & 32 & 96 & 351 & 382 & 10 \\
\hline 5 & 0 & 0 & 0 & 14 & 345 & 18 & 113 & 362 & 355 & 11 \\
\hline 6 & 0 & 0 & 0 & 5 & 356 & 12 & 86 & 324 & 371 & 10 \\
\hline 7 & 0 & 0 & 0 & 6 & 312 & 26 & 95 & 352 & 377 & 9 \\
\hline 8 & 0 & 0 & 0 & 6 & 369 & 20 & 112 & 329 & 365 & 8 \\
\hline 9 & 0 & 0 & 0 & 4 & 372 & 20 & 87 & 355 & 352 & 10 \\
\hline 10 & 0 & 0 & 0 & 4 & 392 & 24 & 120 & 324 & 347 & 3 \\
\hline 11 & 0 & 0 & 0 & 5 & 381 & 22 & 122 & 321 & 369 & 9 \\
\hline 12 & 0 & 0 & 0 & 2 & 367 & 20 & 96 & 345 & 325 & 12 \\
\hline
\end{tabular}

\begin{tabular}{|l|r|r|r|r|r|r|r|r|r|r|}
\hline Média & 0 & 0,00 & 0,00 & 7,25 & 355,50 & 21,42 & 102,67 & 350,42 & 361,33 & 9,17 \\
\hline Temp $^{\circ} \mathrm{C}$ & 22,0 & 22,0 & 22,0 & 23,0 & 25,0 & 25 & 22 & 23,0 & 23,0 & 24,0 \\
\hline $\mathrm{pH}$ & 7 & 7 & 7 & 7 & 7,17 & 7,14 & 7,43 & 7,44 & 7,6 & 7,52 \\
\hline
\end{tabular}

\begin{tabular}{|c|c|c|c|c|c|c|c|c|c|c|}
\hline & & Sistema 2 & $2-\mathrm{C2}$ & Ciliados & & & & & & \\
\hline Amostra & dia 0 & dia 1 . & dia 2 & dia 3 & dia 4 & dia 5 & dia 6 & dia 7 & dia 8 & dia 9 \\
\hline 1 & 0 & 0 & 0 & 12 & 290 & 300 & 420 & 415 & 193 & 15 \\
\hline 2 & 0 & 0 & 0 & 22 & 280 & 400 & 495 & 284 & 248 & 21 \\
\hline 3 & 0 & 0 & 0 & 12 & 310 & 430 & 450 & 422 & 221 & 17 \\
\hline 4 & 0 & 0 & 0 & 15 & 300 & 450 & 495 & 360 & 226 & 15 \\
\hline 5 & 0 & 0 & 0 & 14 & 330 & 400 & 505 & 350 & 203 & 12 \\
\hline 6 & 0 & 0 & 0 & 9 & 360 & 400 & 450 & 360 & 229 & 17 \\
\hline 7 & 0 & 0 & 0 & 7 & 300 & 450 & 412 & 370 & 231 & 22 \\
\hline 8 & 0 & 0 & 0 & 7 & 300 & 400 & 410 & 375 & 227 & 11 \\
\hline 9 & 0 & 0 & 0 & 5 & 300 & 400 & 420 & 360 & 231 & 19 \\
\hline 10 & 0 & 0 & 0 & 6 & 300 & 450 & 390 & 350 & 203 & 20 \\
\hline 11 & 0 & 0 & 0 & 11 & 330 & 455 & 390 & 365 & 239 & 22 \\
\hline 12 & 0 & 0 & 0 & 12 & 320 & 415 & 320 & 360 & 215 & 21 \\
\hline Média & d) & 0,00 & 0,00 & 11,00 & 310,00 & 412,50 & 429,75 & 364,25 & 222.17 & 17,67 \\
\hline $\operatorname{Temp}^{\circ} \mathrm{C}$ & 22,0 & 22,0 & 22,0 & 23,0 & 25,0 & 25 & 22,0 & 23,0 & 23,0 & 24,0 \\
\hline $\mathrm{pH}$ & 7,1 & 7,1 & 7,15 & 7,59 & 7,41 & 7,87 & 8,09 & 7,92 & 8,15 & 8,18 \\
\hline
\end{tabular}

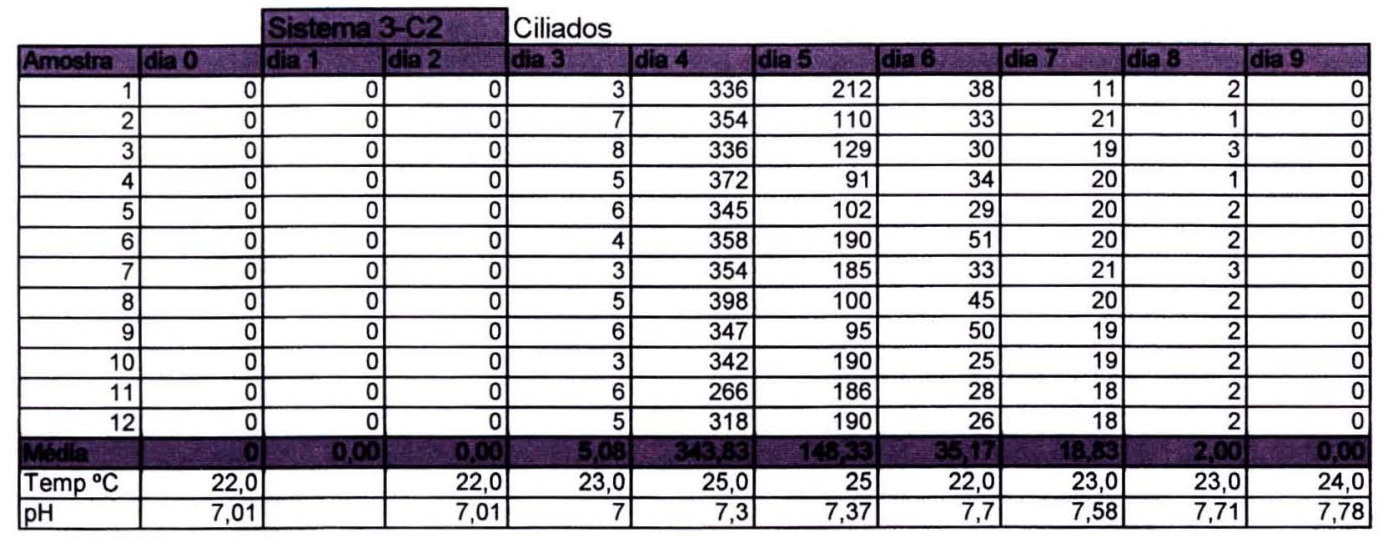


Ciliados

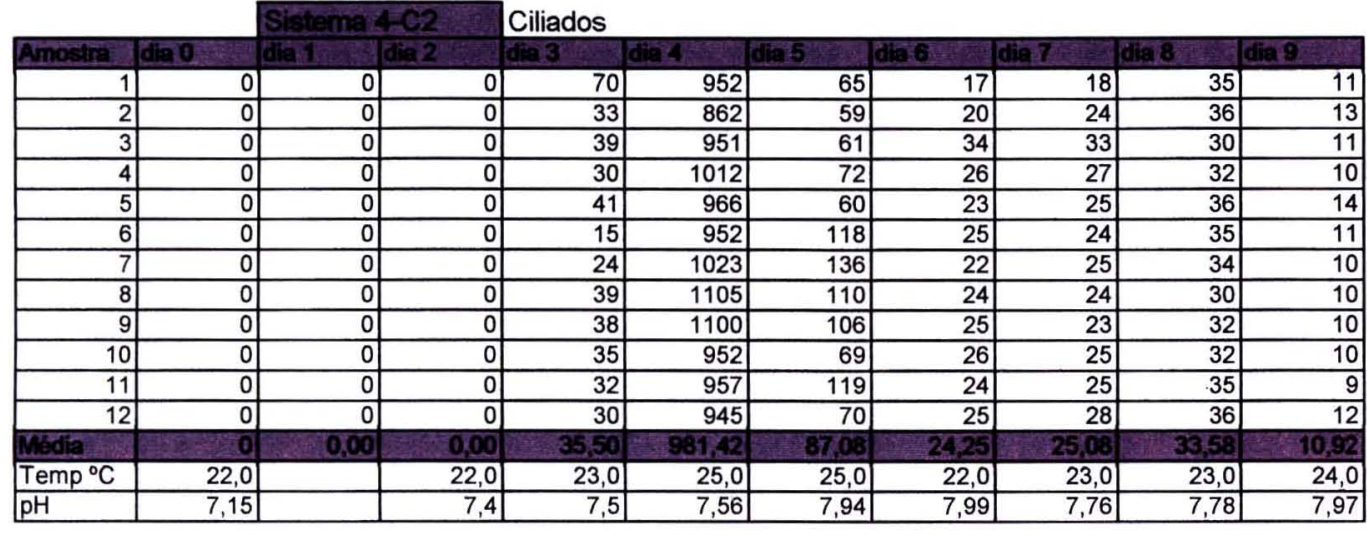

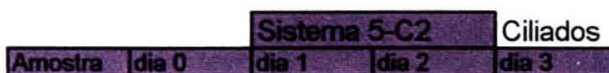

\begin{tabular}{|c|c|c|c|c|c|c|c|c|c|c|}
\hline Amostra & dia 0 & dia 1 & dia 2 & dia 3 . & dia 4 & dia 5 & dia 6 & $\operatorname{dia} 7$ & dia 8 & dia 9 \\
\hline 1 & 0 & 0 & 0 & 0 & 421 & 105 & 83 & 143 & 175 & 0 \\
\hline 2 & 0 & 0 & 0 & 0 & 365 & 97 & 58 & 153 & 217 & 1 \\
\hline 3 & 0 & 0 & 0 & 0 & 412 & 104 & 93 & 105 & 189 & 2 \\
\hline 4 & 0 & 0 & 0 & 0 & 382 & 93 & 52 & 99 & 191 & 1 \\
\hline 5 & 0 & 0 & 0 & 0 & 442 & 98 & 117 & 137 & 186 & 0 \\
\hline 6 & 0 & 0 & 0 & 0 & 425 & 116 & 52 & 122 & 188 & 1 \\
\hline 7 & 0 & 0 & 0 & 0 & 471 & 109 & 75 & 135 & 187 & 2 \\
\hline 8 & 0 & 0 & 0 & 0 & 456 & 98 & 75 & 130 & 201 & 2 \\
\hline 9 & 0 & 0 & 0 & 0 & 392 & 100 & 75 & 130 & 211 & 5 \\
\hline 10 & 0 & 0 & 0 & 0 & 491 & 90 & 76 & 128 & 190 & 3 \\
\hline 11 & 0 & 0 & 0 & 0 & 440 & 100 & 78 & 145 & 192 & 0 \\
\hline 12 & 0 & 0 & 0 & 0 & 421 & 101 & 75 & 140 & 197 & 0 \\
\hline Média & 0 & 0,00 & 0,00 & 0,00 & 426,50 & 100,92 & 75,75 & 130,58 & 193,67 & 1,42 \\
\hline Temp ${ }^{\circ} \mathrm{C}$ & 22,0 & 22,0 & 22,0 & 23,0 & 25,0 & 25,0 & 22,0 & 23,0 & 23,0 & 24,0 \\
\hline $\mathrm{pH}$ & 7,1 & 7,1 & 7,1 & 7,15 & 7,94 & 7,93 & 7,65 & 7,75 & 8,01 & 8,03 \\
\hline
\end{tabular}

\section{Quantidade de microrganismos/substrato DBO de $350 \mathrm{mg} / \mathrm{l}$ aeraçăo continual $3,12 \mathrm{ppm}$ de Cobre no $4^{\circ}$ dia}

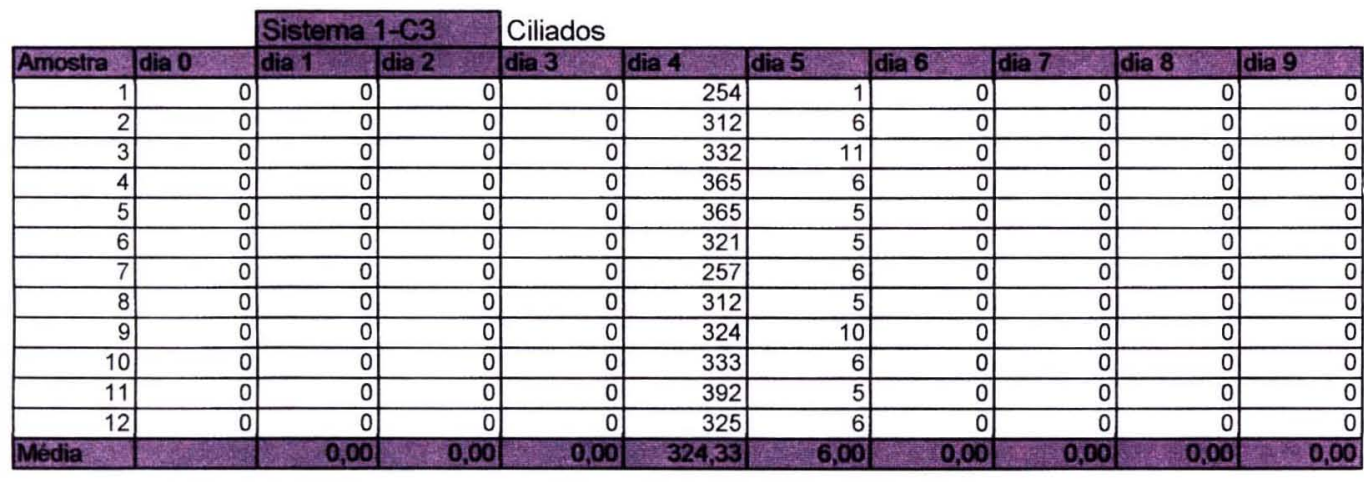

\begin{tabular}{|c|c|c|c|c|c|c|c|c|c|c|}
\hline & & Sistema: & $2-c 3$ & Ciliados & & & & & & \\
\hline Amostra & dia 0 & dia 1 & $\overline{\text { dia } 2}$ & dia 3 & dia 4 & dia 5 & dia 6 & $\overline{\text { dia } 7}$ & dia 8 & dia 9 \\
\hline 1 & 0 & 0 & 0 & 0 & 255 & 0 & 0 & 0 & 0 & 0 \\
\hline 2 & 0 & 0 & 0 & 0 & 265 & 0 & 0 & 0 & 0 & 0 \\
\hline 3 & 0 & 0 & 0 & 0 & 245 & 0 & 0 & 0 & 0 & 0 \\
\hline 4 & 0 & 0 & 0 & 0 & 312 & 0 & 0 & 0 & 0 & 0 \\
\hline 5 & 0 & 0 & 0 & 0 & 236 & 0 & 0 & 0 & 0 & 0 \\
\hline 6 & 0 & 0 & 0 & 0 & 333 & 0 & 0 & 0 & 0 & 0 \\
\hline 7 & 0 & 0 & 0 & 0 & 258 & 0 & 0 & 0 & 0 & 0 \\
\hline 8 & 0 & 0 & 0 & 0 & 254 & 0 & 0 & 0 & 0 & 0 \\
\hline 9 & 0 & 0 & 0 & 0 & 269 & 0 & 0 & 0 & 0 & 0 \\
\hline 10 & 0 & 0 & 0 & 0 & 271 & 0 & 0 & 0 & 0 & 0 \\
\hline 11 & 0 & 0 & 0 & 0 & 280 & 0 & 0 & 0 & 0 & 0 \\
\hline 12 & 0 & 0 & 0 & 0 & 321 & 0 & 0 & 0 & 0 & 0 \\
\hline Média & & 0,00 & 0,00 & 0,00 & 274,92 & 0,00 & 0,00 & 0,00 & 0,00 & 0,00 \\
\hline Temp ${ }^{\circ} \mathrm{C}$ & 20,0 & 20,0 & 21,0 & 23,0 & 23,0 & 22,0 & 22,0 & 23,0 & 22,0 & 23,0 \\
\hline $\mathrm{pH}$ & 7 & 7,07 & 7,54 & 7,39 & 7,05 & 7,16 & 7,3 & 7,41 & 7,4 & 7,87 \\
\hline
\end{tabular}




\begin{tabular}{|c|c|c|c|c|c|c|c|c|c|c|}
\hline tra & dia. 0 & dia 1 & dia 2 & dia 3 & dia 4 & 13 & 6 & a. & dia 8. & dia 9 \\
\hline 1 & 0 & 0 & $\begin{array}{r}0 \\
\end{array}$ & 1 & 352 & 15 & 0 & 0 & 0 & 0 \\
\hline 2 & 0 & 0 & 0 & 2 & 412 & 10 & 0 & 20 & 0 & 0 \\
\hline 3 & 0 & 0 & 0 & 11 & 558 & 6 & 0 & 20 & 0 & 0 \\
\hline 4 & 0 & 0 & 0 & 6 & 550 & 5 & 0 & 15 & 0 & 0 \\
\hline 5 & 0 & 0 & 0 & 5 & 512 & 12 & 0 & 15 & 0 & 0 \\
\hline 6 & 0 & 0 & 0 & 4 & 492 & 15 & 0 & 10 & 0 & 0 \\
\hline 7 & 0 & 0 & 0 & 6 & 442 & 10 & 0 & 10 & 0 & 0 \\
\hline 8 & 0 & 0 & 0 & 12 & 525 & 8 & 0 & 10 & 0 & 0 \\
\hline 9 & 0 & 0 & 0 & 21 & 575 & 10 & 0 & 10 & 0 & 0 \\
\hline 10 & 0 & 0 & 0 & 21 & 592 & 9 & 0 & 12 & 0 & 0 \\
\hline 11 & 0 & 0 & 0 & 33 & 445 & 10 & 0 & 10 & 0 & 0 \\
\hline 12 & 0 & 0 & 0 & 21 & 569 & 10 & 0 & 12 & 0 & 0 \\
\hline Média & & 0,00 & 0,00 & 11,92 & 502,00 & 10,00 & 0,00 & 12,00 & 0,00 & 0,00 \\
\hline Temp $^{\circ} \mathrm{C}$ & 20,0 & 20,0 & 21,0 & 23,0 & 23,0 & 22,0 & 22,0 & 23,0 & 22,0 & 23,0 \\
\hline $\mathrm{pH}$ & 7 & 7,03 & 6,41 & 7,45 & 7,77 & 7,94 & 7,89 & 8,11 & 8,08 & 8,1 \\
\hline
\end{tabular}

0
0,00
0,00
11,92
502,00
10,00
0,00
12,00
0,00
0,00

\begin{tabular}{|c|c|c|c|c|c|c|c|c|c|c|}
\hline & & Sistema & $4-C 3$ & Ciliados & & & & & & \\
\hline Amostra & dia 0 & dia 1 & dia 2 & dia 3 & dia 4 & dia 5. & dia 6 & dia 7 & dia 8 & dia 9 \\
\hline 1 & 1 & 0 & 0 & \begin{tabular}{|l|}
121 \\
\end{tabular} & 312 & 0 & 0 & 0 & 0 & 0 \\
\hline 2 & 0 & 0 & 0 & 132 & 662 & 0 & 0 & 0 & 0 & 0 \\
\hline 3 & 0 & 0 & 0 & 144 & 654 & 0 & 0 & 0 & 0 & 0 \\
\hline 4 & 0 & 0 & 0 & 212 & 612 & 0 & 0 & 0 & 0 & 0 \\
\hline 5 & 0 & 0 & 0 & 192 & 712 & 0 & 0 & 0 & 0 & 0 \\
\hline 6 & 0 & 0 & 0 & 201 & 772 & 0 & 0 & 0 & 0 & 0 \\
\hline 7 & 0 & 0 & 0 & 192 & 721 & 0 & 0 & 0 & 0 & 0 \\
\hline 8 & 0 & 0 & 0 & 222 & 701 & 0 & 0 & 0 & 0 & 0 \\
\hline 9 & 0 & 0 & 0 & 123 & 655 & 0 & 0 & 0 & 0 & 0 \\
\hline 10 & 0 & 0 & 0 & 185 & 647 & 0 & 0 & 0 & 0 & 0 \\
\hline 11 & 0 & 0 & 0 & 192 & 712 & 0 & 0 & 0 & 0 & 0 \\
\hline 12 & 0 & 0 & 0 & 171 & 687 & 0 & 0 & 0 & 0 & 0 \\
\hline Média & & 0,00 & 0,00 & 173,92 & 653,92 & 0,00 & 0,00 & 0,00 & 0,00 & 0,00 \\
\hline $\operatorname{Temp}^{\circ} \mathrm{C}$ & 20,0 & 20,0 & 21,0 & 23,0 & 23,0 & 22,0 & 22,0 & 23,0 & 22,0 & 23,0 \\
\hline $\mathrm{pH}$ & 7,1 & 7,28 & 7,12 & 7,42 & 7,69 & 7,84 & 7,9 & 7,94 & \begin{tabular}{l|l|}
7,96 & \\
\end{tabular} & 7,87 \\
\hline
\end{tabular}

\begin{tabular}{|c|c|c|c|c|c|c|c|c|c|c|}
\hline & & Sistema 5 & $5-63$ & Ciliados & & & & & & \\
\hline Amostra & dia 0 & dia 1 & dia 2 & dia 3 & dia 4 & dia 5 & dia 6 & dia 7 & dia 8. & dia 9 \\
\hline 1 & 0 & 0 & 0 & 192 & 812 & 0 & 0 & 0 & 0 & 0 \\
\hline 2 & 0 & 0 & 0 & 255 & 808 & 0 & 0 & 0 & 0 & 0 \\
\hline 3 & 0 & 0 & 0 & 265 & 775 & 0 & 0 & 0 & 0 & 0 \\
\hline 4 & 0 & 0 & 0 & 212 & 852 & 0 & 0 & 0 & 0 & 0 \\
\hline 5 & 0 & 0 & 0 & 395 & 795 & 0 & 0 & 0 & 0 & 0 \\
\hline 6 & 0 & 0 & 0 & 365 & 756 & 0 & 0 & 0 & 0 & 0 \\
\hline 7 & 0 & 0 & 0 & 225 & 812 & 0 & 0 & 0 & 0 & 0 \\
\hline 8 & 0 & 0 & 0 & 312 & 852 & 0 & 0 & 0 & 0 & 0 \\
\hline 9 & 0 & 0 & 0 & 345 & 779 & 0 & 0 & 0 & 0 & 0 \\
\hline 10 & 0 & 0 & 0 & 332 & 792 & 0 & 0 & 0 & 0 & 0 \\
\hline 11 & 0 & 0 & 0 & 369 & 825 & 0 & 0 & 0 & 0 & 0 \\
\hline 12 & 0 & 0 & 0 & 354 & 921 & 0 & 0 & 0 & 0 & 0 \\
\hline Média : & & 0.00 & 0,00 & 301.75 & 814,92 & 0.00 & 0.00 & 0,00 & 0.001 & 0.00 \\
\hline $\operatorname{Temp}^{\circ} \mathrm{C}$ & 20,0 & 20,0 & 21,0 & 23,0 & 23,0 & 22,0 & 22,0 & 23,0 & 22,0 & 23,0 \\
\hline $\mathrm{pH}$ & 7,2 & 7,33 & 7,55 & 7,53 & 7,71 & 8,03 & 8,07 & 8,12 & 8,12 & 8 \\
\hline
\end{tabular}


Experimento II

Quantidade de microrganismos/substrato DBO $350 \mathrm{mg} /$ /aeraçăo continua

\begin{tabular}{|c|c|c|c|c|c|c|c|c|c|c|c|}
\hline \multirow{2}{*}{ Amostra } & \multirow[b]{2}{*}{ dia 0} & \multicolumn{2}{|l|}{ Sistema 1 } & \multicolumn{7}{|c|}{ Bactérias Filamentosas } & \multirow[b]{2}{*}{0} \\
\hline & & dia 1 & dia 2 & dia 3 & dia 4 & dia 5 & $\operatorname{dia} 6$ & dia 7 & dia 8 & dia 9 & \\
\hline 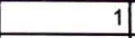 & 0 & 1 & 2 & 25 & 8 & 6 & 25 & 2 & 7 & 1 & 2,00 \\
\hline 2 & 0 & 2 & 1 & 16 & 8 & 8 & 30 & 2 & 6 & 5 & 2,25 \\
\hline 3 & 0 & 1 & 2 & 18 & 5 & 2 & 30 & 2 & 2 & 1 & 15,33 \\
\hline 4 & 0 & 1 & 0 & 12 & 9 & 3 & 32 & 1 & 25 & 4 & 8,00 \\
\hline 5 & 0 & 2 & 2 & 4 & 8 & 6 & 19 & 1 & 22 & 4 & 4,92 \\
\hline 6 & 0 & 2 & 2 & 6 & 5 & 9 & 18 & 2 & 19 & 5 & 27,25 \\
\hline 7 & 0 & 3 & 3 & 11 & 8 & 2 & 33 & 1 & 16 & 4 & 1,33 \\
\hline 8 & 0 & 5 & 4 & 23 & 8 & 8 & 35 & 1 & 7 & 6 & 12,50 \\
\hline 9 & 0 & 1 & 6 & 22 & 10 & 8 & 20 & 1 & 11 & 4 & 3,83 \\
\hline 10 & 0 & 1 & 2 & 9 & 9 & 5 & 35 & 1 & 8 & 4 & \\
\hline 11 & 0 & 2 & 2 & 15 & 9 & 2 & 35 & 1 & 18 & 4 & \\
\hline 12 & 0 & 3 & 1 & 23 & 9 & 0 & 15 & 1 & 9 & 4 & \\
\hline Média & 0 & 2,00 & 2,25 & 15,33 & 8,00 & 4,92 & 27,25 & 1,33 & 12,50 & 3,83 & \\
\hline Temp $^{\circ} \mathrm{C}$ & 25 & 22,0 & 22,0 & 22,0 & 23,5 & 22,5 & 24,0 & 23,0 & 23,0 & 23,0 & \\
\hline \multirow[t]{2}{*}{$\mathrm{pH}$} & 7,01 & \begin{tabular}{|r|}
7,15 \\
\end{tabular} & 7,23 & \begin{tabular}{|r|}
7,37 \\
\end{tabular} & 7,55 & \begin{tabular}{|l|}
7,62 \\
\end{tabular} & 7,77 & 7,8 & 7,89 & 7,9 & \\
\hline & & \multicolumn{2}{|c|}{ Sistema 2} & \multicolumn{7}{|c|}{ Bactérias Filamentosas } & \\
\hline Amostra & $\operatorname{dia} 0$ & \begin{tabular}{|l|l} 
dia 1 & \\
\end{tabular} & dia 2 & dia 3 & dia 4 & $\operatorname{dia} 5$ & $\operatorname{dia} 6$ & dia 7 & dia 8 & dia 9 & 0 \\
\hline 1 & 0 & 1 & 0 & 4 & 0 & 2 & 1 & 0 & 0 & 1 & 2,00 \\
\hline 2 & 0 & 2 & 2 & 5 & 0 & 3 & 1 & 0 & 0 & 1 & 2,75 \\
\hline 3 & 0 & 2 & 3 & 3 & 0 & 5 & 5 & 0 & 0 & 1 & 4,00 \\
\hline 4 & 0 & 0 & 2 & 2 & 0 & 4 & 6 & 0 & 0 & 0 & 1,00 \\
\hline 5 & 0 & 2 & 2 & 2 & 2 & 4 & 5 & 0 & 1 & 0 & 2,50 \\
\hline 6 & 0 & 4 & 2 & 2 & 5 & 3 & 1 & 0 & 1 & 0 & 2,58 \\
\hline 7 & 0 & 5 & 2 & 5 & 2 & 2 & 1 & 0 & 1 & 1 & 0,00 \\
\hline 8 & 0 & 3 & 4 & 5 & 1 & 1 & 1 & 0 & 1 & 1 & 0,58 \\
\hline 9 & 0 & 1 & 2 & 5 & 1 & 2 & 1 & 0 & 1 & 1 & 0,83 \\
\hline 10 & 0 & 0 & 3 & 5 & 0 & 2 & 5 & 아 & 1 & 1 & \\
\hline 11 & 0 & 2 & 5 & 6 & 0 & 2 & 2 & 0 & 1 & 2 & \\
\hline 12 & 0 & 2 & 6 & 4 & 1 & 0 & 2 & 0 & 0 & 1 & \\
\hline Média & 0 & 2,00 & 2,75 & 4,00 & 1,00 & 2,50 & 2,58 & 0,00 & 0,58 & 0,83 & \\
\hline Temp $^{\circ} \mathrm{C}$ & 25 & 22,0 & 22,0 & 22,0 & 23,5 & 23,5 & 24,0 & 23,0 & 23,0 & 23,0 & \\
\hline $\mathrm{pH}$ & 6,95 & 7,1 & 7,18 & 7,22 & 7,23 & 7,06 & 7,52 & 7,7 & 7,78 & 7,9 & \\
\hline
\end{tabular}

\begin{tabular}{|c|c|c|c|c|c|c|c|c|c|c|}
\hline & & Sistema 3 & & Bactérias & Filamentc & osas & & & & \\
\hline Amostra & dia 0 & dia 1 & dia 2 & dia 3 & dia 4 & dia 5 & dia 6 & dia 7 & dia 8 & dia 9 \\
\hline 1 & 0 & 0 & 4 & 3 & 0 & 125 & 220 & 325 & \begin{tabular}{|l|}
505 \\
\end{tabular} & \begin{tabular}{|r}
515 \\
\end{tabular} \\
\hline 2 & 0 & 0 & 1 & 4 & 0 & 100 & 205 & 360 & 432 & 456 \\
\hline 3 & 0 & 2 & 1 & 5 & 0 & 90 & 190 & 345 & 500 & 553 \\
\hline 4 & 0 & 2 & 1 & 8 & 0 & 95 & 180 & 369 & 260 & 356 \\
\hline 5 & 0 & 1 & 1 & 9 & 3 & 86 & 160 & 387 & 368 & 446 \\
\hline 6 & 0 & 5 & 4 & 9 & 0 & 75 & 105 & 398 & 450 & 489 \\
\hline 7 & 0 & 6 & 2 & 6 & 1 & 90 & 200 & 356 & 440 & 470 \\
\hline 8 & 0 & 1 & 2 & 6 & 1 & 95 & 190 & 297 & 442 & 506 \\
\hline 9 & 0 & 2 & 4 & 9 & 2 & 99 & 170 & 330 & 556 & 527 \\
\hline 10 & 0 & 2 & 2 & 10 & 1 & 87 & 175 & 336 & 456 & 390 \\
\hline 11 & 0 & 1 & 2 & 6 & 2 & 66 & 190 & 358 & 389 & 550 \\
\hline 12 & 0 & 2 & 2 & 9 & 2 & 54 & 190 & 332 & 365 & 559 \\
\hline Média & of & 2,00 & 2,17 & 7,00 & 1,00 & 88,50 & 181,25 & 349,42 & 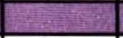 & 484,75 \\
\hline Temp ${ }^{\circ} \mathrm{C}$ & 25,0 & 22 & 22,0 & 22,0 & 23,5 & 22,5 & 24,0 & 23,0 & 23,0 & 23,0 \\
\hline $\mathrm{pH}$ & 6,95 & 7,01 & 7 & 6,9 & 6,81 & 7,81 & 8,04 & 8,1 & 8,21 & 8,23 \\
\hline
\end{tabular}

\begin{tabular}{|c|c|c|c|c|c|c|c|c|c|c|}
\hline & & Sistema 4 & & Bactérias & Filamentc & osas & & & & \\
\hline Amostra & dia 0 & dia 1 & dia 2 & dia 3 & \begin{tabular}{|l|l|} 
dia 4 \\
\end{tabular} & dia 5 & dia 6 & dia 7 & dia 8 & dia 9 \\
\hline 1 & 0 & 2 & 1 & 10 & 0 & 2 & 2 & 1 & 2 & 2 \\
\hline 2 & 0 & 3 & 1 & 12 & 0 & 3 & 2 & 1 & 2 & 4 \\
\hline 3 & 0 & 4 & 1 & 8 & 0 & 2 & 2 & 1 & 2 & 2 \\
\hline 4 & 0 & 2 & 1 & 6 & 0 & 6 & 4 & 0 & 2 & 4 \\
\hline 5 & 0 & 3 & 2 & 5 & 0 & 5 & 4 & 1 & 2 & 2 \\
\hline 6 & 0 & 2 & 2 & 9 & 0 & 5 & 2 & 0 & 2 & 4 \\
\hline 7 & 0 & 0 & 5 & 8 & 0 & 2 & 3 & 1 & 2 & 4 \\
\hline 8 & 0 & 0 & 2 & 7 & 0 & 2 & 4 & 0 & 3 & 5 \\
\hline 9 & 0 & 2 & 2 & 2 & 0 & 0 & 4 & 0 & 2 & 1 \\
\hline 10 & 0 & 3 & 3 & 3 & 0 & 1 & 2 & 0 & 2 & 1 \\
\hline 11 & 0 & 2 & 1 & 5 & 0 & 1 & 2 & 1 & 2 & 1 \\
\hline 12 & 0 & 1 & 9 & 9 & 0 & 1 & 6 & 1 & 1 & 1 \\
\hline Média & 0 & 2,00 & 2,50 & 7,00 & 0,00 & 2,50 & 3,08 & 0,58 & 2,00 & 2,58 \\
\hline Temp ${ }^{\circ} \mathrm{C}$ & 25,0 & 22 & 22,0 & 22,0 & 23,5 & 22,5 & 24,0 & 23,0 & 23,0 & 23,0 \\
\hline $\mathrm{pH}$ & 6,87 & 6,9 & 7,15 & 7,55 & 7,85 & 7,88 & 8,06 & 8,1 & 8,16 & 8,2 \\
\hline
\end{tabular}




\begin{tabular}{|c|c|c|c|c|c|c|c|c|c|c|}
\hline Amostra & dia 0 & dia 1 & dia 2 & dia 3 & dia 4 & dia 5 & dia 6 & $\operatorname{dia} 7$ & dia 8 & dia? 9 \\
\hline 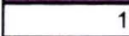 & 0 & 1 & 1 & 5 & 0 & 2 & 2 & 1 & 5 & \\
\hline 2 & 0 & 2 & 2 & 5 & 2 & 3 & 3 & 1 & 4 & \\
\hline 3 & 0 & 1 & 2 & 4 & 2 & 2 & 5 & 1 & 3 & \\
\hline 4 & 0 & 1 & 3 & 5 & 1 & 0 & 6 & 2 & 5 & \\
\hline 5 & 0 & 1 & 2 & 5 & 2 & 1 & 2 & 4 & 4 & \\
\hline 6 & 0 & 1 & 2 & 5 & 3 & 0 & 2 & 2 & 4 & \\
\hline 7 & 0 & 2 & 0 & 6 & 1 & 1 & 0 & 2 & 2 & \\
\hline 8 & 0 & 2 & 0 & 6 & 1 & 1 & 0 & 2 & 4 & \\
\hline 9 & 0 & 2 & 2 & 2 & 1 & 1 & 0 & 2 & 4 & \\
\hline 10 & 0 & 3 & 3 & 6 & 0 & 1 & 0 & 1 & 5 & \\
\hline 11 & 0 & 4 & 0 & 6 & 0 & 0 & 0 & 1 & 5 & \\
\hline 12 & 0 & 4 & 2 & 5 & 0 & 0 & 0 & 1 & 4 & \\
\hline Média & 0 & 2,00 & 1,58 & 5,00 & 1,08 & 1,00 & 1,67 & 1,67 & 4,08 & 4,1 \\
\hline Temp ${ }^{\circ} \mathrm{C}$ & 25,0 & 22 & 22,0 & 22,0 & 23,5 & 22,5 & 24,0 & 23,0 & 23,0 & 23 \\
\hline $\mathrm{pH}$ & 6,92 & 7 & 7,25 & 7.6 & 7,85 & 7,63 & 7,83 & 7,9 & 7,92 & 7,9 \\
\hline
\end{tabular}

\begin{tabular}{|c|c|c|c|c|c|c|c|c|c|c|c|}
\hline & & Sistema $1-\mathrm{C}$ & Cl & Bactérias $\mathrm{F}$ & ilamentosa & & & & & & \\
\hline Amostra & dia 0 & dia 1 & dia 2 & dia 3 & dia 4 & dia 5 & dia 6 & dia 7 & dia 8 & dia 9 & \\
\hline 1 & 0 & 2 & 1 & 1 & 4 & 7 & 2 & 1 & 4 & 1 & \\
\hline 2 & 0 & 2 & 2 & 3 & 2 & 7 & 2 & 1 & 0 & 2 & 4,0 \\
\hline 3 & 0 & 2 & 6 & 4 & 6 & 13 & 2 & 0 & 2 & 2 & \\
\hline 4 & 0 & 2 & 5 & 4 & 2 & 14 & 2 & 0 & 1 & 1 & \\
\hline 5 & 0 & 1 & 6 & 2 & 2 & 8 & 2 & 1 & 0 & 1 & 10,0 \\
\hline 6 & 0 & 2 & 5 & 1 & 5 & 11 & 2 & 1 & 0 & 1 & \\
\hline 7 & 0 & 2 & 4 & 4 & 5 & 10 & 2 & 1 & 0 & 1 & \\
\hline 8 & 0 & 2 & 2 & 2 & 4 & 9 & 2 & 1 & 2 & 1 & \\
\hline 9 & 0 & 2 & 2 & 2 & 5 & 8 & 2 & 2 & 2 & 2 & \\
\hline 10 & 0 & 2 & 6 & 2 & 6 & 10 & 2 & 2 & 0 & 1 & \\
\hline 11 & 0 & 2 & 8 & 2 & 4 & 11 & 2 & 2 & 2 & 0 & \\
\hline 12 & 0 & 2 & 2 & 2 & 4 & 12 & 2 & 4 & 4 & 0 & \\
\hline Média & $\underline{0}$ & 0,00 & 4,08 & 2,42 & 4,08 & 10,00 & 2,00 & 1,33 & 1,42 & 1,08 & \\
\hline Temp ${ }^{\circ} \mathrm{C}$ & 25 & 22,0 & 22,0 & 23,0 & 23,5 & 22,5 & 24,0 & 24,0 & 23,0 & 23,0 & \\
\hline $\mathrm{pH}$ & 7,05 & 7,2 & 7,2 & 7,25 & 7,79 & 7,66 & 7,92 & 7,77 & 7,9 & 8 & \\
\hline
\end{tabular}

\begin{tabular}{|c|c|c|c|c|c|c|c|c|c|c|}
\hline & & Sistema $2-1$ & & ctérias F & Fila & & & & & \\
\hline Amostra & dia 0 & dia 1 & dia 2 & $\longdiv { d i a 3 }$ & dia 4 & dia 5 & dia 6 & dia 7 & dia 8 & dia 9 \\
\hline 1 & 0 & 2 & 1 & 5 & 1 & 5 & 2 & 3 & 20 & 0 \\
\hline 2 & 0 & 4 & 2 & 3 & 1 & 2 & 2 & 4 & 6 & 0 \\
\hline 3 & 0 & 2 & 3 & 5 & 1 & 11 & 4 & 2 & 8 & 1 \\
\hline 4 & 0 & 2 & 2 & 4 & 1 & 2 & 5 & 3 & 7 & 1 \\
\hline 5 & 0 & 2 & 2 & 3 & 1 & 2 & 7 & 3 & 5 & 0 \\
\hline 6 & 0 & 2 & 2 & 2 & 1 & 6 & 2 & 3 & 6 & 0 \\
\hline 7 & 0 & 5 & 2 & 3 & 1 & 8 & 2 & 3 & 8 & 0 \\
\hline 8 & 0 & 2 & 2 & 2 & 1 & 9 & 5 & 2 & 9 & 1 \\
\hline 9 & 0 & 5 & 5 & 4 & 1 & 10 & 3 & 1 & 8 & 2 \\
\hline 10 & 0 & 6 & 6 & 5 & 1 & 16 & 2 & 2 & 5 & 2 \\
\hline 11 & 0 & 6 & 6 & 2 & 2 & 12 & 6 & 3 & 6 & 1 \\
\hline 12 & 0 & 2 & 6 & 3 & 1 & 6 & 2 & 3 & 4 & 1 \\
\hline Média & 0 & 3,33 & 3,25 & 3,42 & 1,08 & 7,42 & 3,50 & 2,67 & 7,67 & 0.75 \\
\hline Temp ${ }^{\circ} \mathrm{C}$ & 25 & 22,0 & 22,0 & 23,0 & 23,5 & 22,5 & 24,0 & 23,0 & 23,0 & 23,0 \\
\hline $\mathrm{pH}$ & 6,96 & 7 & 7 & 7,02 & 7,48 & 7,66 & 7,91 & 8 & 8,02 & 8 \\
\hline
\end{tabular}

\begin{tabular}{|c|c|c|c|c|c|c|c|c|c|c|}
\hline & & & & Bactérias F & Filamentosas & & & & & \\
\hline mostra & dia. 0 & dia 1 & dia 2 & dias 3 & dia 4 & dia 5 & dia 8 & dia 7. & dila 8. & diag \\
\hline 1 & 0 & 4 & 1 & 4 & 1 & 12 & 9 & 4 & 6 & 0 \\
\hline 2 & 0 & 4 & 2 & 5 & 1 & 15 & 8 & 2 & 6 & 2 \\
\hline 3 & 0 & 1 & 2 & 4 & 1 & 12 & 9 & 2 & 4 & 2 \\
\hline 4 & 0 & 4 & 3 & 4 & 1 & 10 & 10 & 2 & 4 & 0 \\
\hline 5 & 0 & 6 & 3 & 3 & 1 & 10 & 7 & 2 & 4 & 0 \\
\hline 6 & 0 & 4 & 3 & 3 & 1 & 10 & 9 & 2 & 4 & 2 \\
\hline 7 & 0 & 4 & 2 & 4 & 1 & 16 & 9 & 2 & 6 & 1 \\
\hline 8 & 0 & 4 & 2 & 5 & 1 & 9 & 8 & 1 & 5 & 1 \\
\hline 9 & 0 & 2 & 2 & 4 & 1 & 11 & 13 & 2 & 6 & 1 \\
\hline 10 & 0 & 6 & 2 & 4 & 1 & 11 & 6 & 1 & 5 & 1 \\
\hline 11 & 0 & 8 & 2 & 4 & 1 & 12 & 9 & 2 & 6 & 1 \\
\hline 12 & 0 & 2 & 1 & 4 & 1 & 10 & 12 & 2 & 6 & \\
\hline dila: & 0 & 4,08 & 2.08 & 4,00 & 1,00 & 11,50 & 9,08 & 2,00 & 5,17 & 1,00 \\
\hline $\operatorname{Temp}^{\circ} \mathrm{C}$ & 25 & 22,0 & 22,0 & 23,0 & 23,5 & 22,5 & 24,0 & 23,0 & 23,0 & 23,0 \\
\hline $\mathrm{pH}$ & 6,95 & 7,25 & 7,35 & 7,59 & 7,7 & $\begin{array}{l}7,73 \\
\end{array}$ & 8,03 & 8 & 8,02 & 8,04 \\
\hline
\end{tabular}




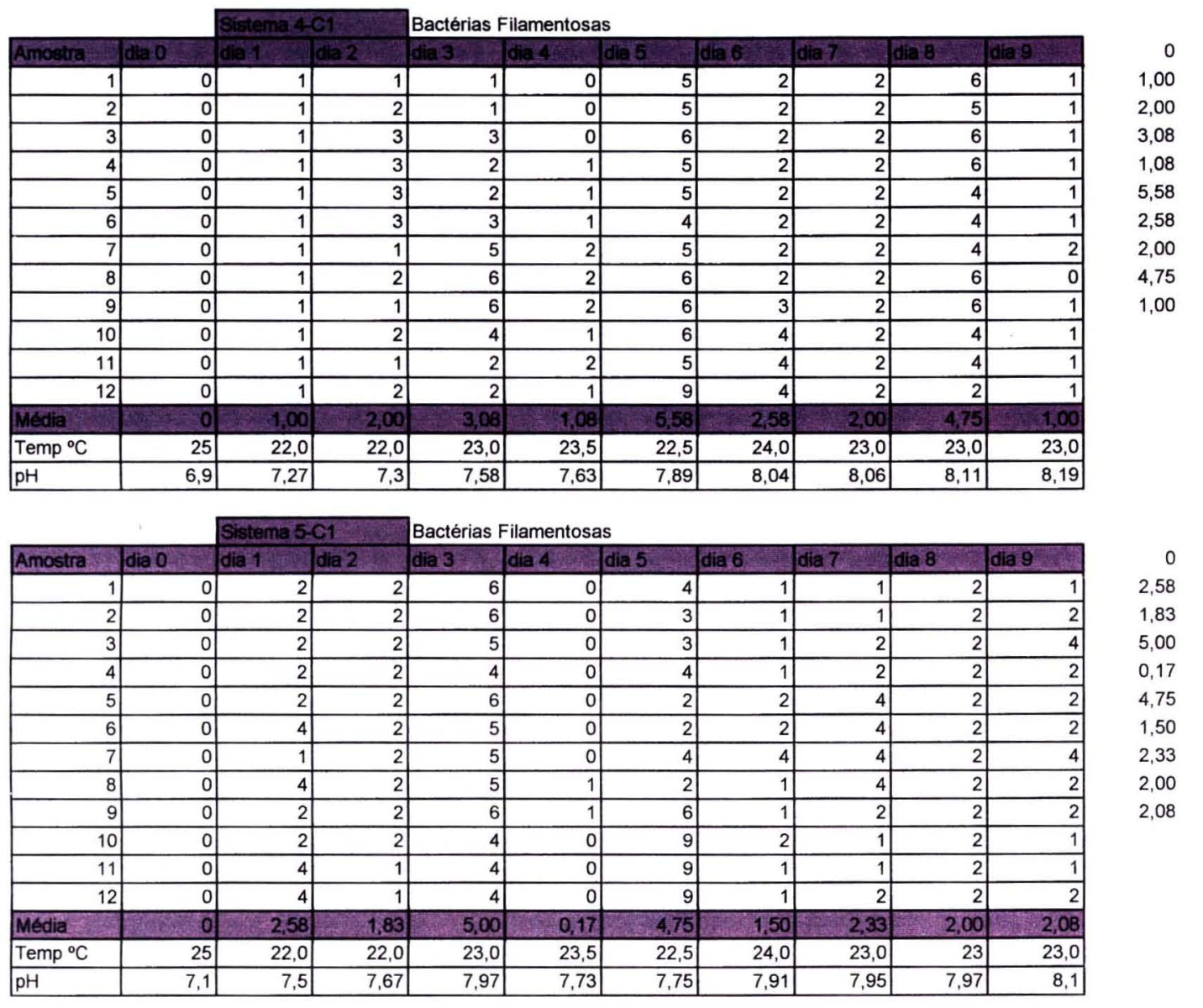

\begin{tabular}{|c|c|c|c|c|c|c|c|c|c|c|c|}
\hline & & \multicolumn{2}{|c|}{ Sistema $10-2$} & \multicolumn{7}{|c|}{ Bactérias Filamentosas } & \multirow[b]{2}{*}{0} \\
\hline Amostra & dia 0 & dia 1 & dia 2 & dia 3 & dia $4 \quad$ & dia 5 & dia 6 & $\operatorname{dia} 7$ & dia 8 & dia 9 & \\
\hline 1 & 0 & 2 & 2 & & 3 & 0 & 3 & 1 & 1 & 0 & 2,00 \\
\hline 2 & 0 & 2 & 2 & 4 & 2 & 0 & 3 & 1 & 1 & 0 & 2,08 \\
\hline 3 & 0 & 2 & 2 & 1 & 2 & 0 & 3 & 1 & 1 & 0 & 3,27 \\
\hline 4 & 0 & 2 & 2 & 1 & 1 & 0 & 3 & 1 & 1 & 0 & 2,00 \\
\hline 5 & 0 & 3 & 2 & 7 & 1 & 0 & 3 & 0 & 1 & 0 & 0,00 \\
\hline 6 & 0 & 2 & 3 & 6 & 3 & 0 & 3 & 0 & 1 & 0 & 3,00 \\
\hline 7 & 0 & 2 & 2 & 3 & 2 & 0 & 3 & 1 & 1 & 0 & 0,50 \\
\hline 8 & 0 & 2 & 4 & 4 & 2 & 0 & 3 & 1 & 1 & 0 & 1,00 \\
\hline 9 & 0 & 2 & 2 & 5 & 2 & 0 & 3 & 0 & 1 & 0 & 0,33 \\
\hline 10 & 0 & 2 & 2 & 2 & 2 & 0 & 3 & 0 & 1 & 1 & \\
\hline 11 & 0 & 3 & 1 & 2 & 2 & 0 & 2 & 0 & 1 & 2 & \\
\hline 12 & 0 & 0 & 1 & 1 & 2 & 0 & 4 & 0 & 1 & 1 & \\
\hline Média & 0 & 2,00 & 2,08 & 3,27 & 2,00 & 0,00 & 3,00 & 0,50 & 1,00 & 0,33 & \\
\hline $\operatorname{Temp}^{\circ} \mathrm{C}$ & 25 & 22,0 & 22,0 & 23,0 & 23,5 & 22,5 & 24,0 & 23,0 & 23,0 & 23,0 & \\
\hline $\mathrm{pH}$ & 6,96 & 7 & 7 & 7 & 7,78 & 7,61 & 7,85 & 7,89 & 7,9 & 8 & \\
\hline & & \multicolumn{2}{|c|}{ Sistema $2-\mathrm{C}_{2}$} & \multicolumn{7}{|c|}{ Bactérias Filamentosas } & \\
\hline Amostra & dia 0 & dia 1 & dia 2 & dia 3 & dia 4 & dia 5. & dia 6 & dia 7 . & dia 8 & dia 9 & 0 \\
\hline 1 & 0 & 1 & 1 & 2 & 0 & 0 & 0 & 1 & 2 & 1 & 1,83 \\
\hline 2 & 0 & 1 & 1 & & 0 & 0 & 0 & 1 & 3 & 3 & 1,33 \\
\hline 3 & 0 & 2 & 1 & 2 & 0 & 0 & 0 & 3 & 2 & 4 & 2,09 \\
\hline 4 & 0 & 2 & 1 & 2 & 0 & 0 & 0 & 1 & 2 & 2 & 0,00 \\
\hline 5 & 0 & 2 & 1 & 2 & 0 & 0 & 0 & 1 & 2 & 2 & 0,00 \\
\hline 6 & 0 & 1 & 1 & 2 & 0 & 0 & 0 & 1 & 2 & 2 & 0,00 \\
\hline 7 & 0 & 2 & 2 & 2 & 0 & 0 & 0 & 0 & 2 & 0 & 1,17 \\
\hline 8 & 0 & 2 & 1 & 2 & 0 & 0 & 0 & 0 & 2 & 0 & 2,08 \\
\hline 9 & 0 & 1 & 2 & 3 & 0 & 0 & 0 & 0 & 2 & 0 & 1,67 \\
\hline 10 & 0 & 2 & 1 & 2 & 0 & 0 & 0 & 4 & 2 & 2 & \\
\hline 11 & 0 & 3 & 2 & 2 & 0 & 0 & 0 & 1 & 2 & 2 & \\
\hline 12 & 0 & 3 & 2 & 2 & 0 & 0 & 0 & 1 & 2 & 2 & \\
\hline Média & 0 & 1,83 & 1,33 & 2,09 & 0,00 & 0,00 & 0,00 & 1,17 & 2,08 & 1,67 & \\
\hline Temp ${ }^{\circ} \mathrm{C}$ & 25 & 22,0 & 22,0 & 23,0 & 23,5 & 22,5 & 24,0 & 23,0 & 23,0 & 23,0 & \\
\hline pH & 7 & 7,1 & 7,15 & 7,59 & \begin{tabular}{|l|}
7,64 \\
\end{tabular} & 7,71 & 7,91 & 8 & 8,03 & 8,18 & \\
\hline
\end{tabular}




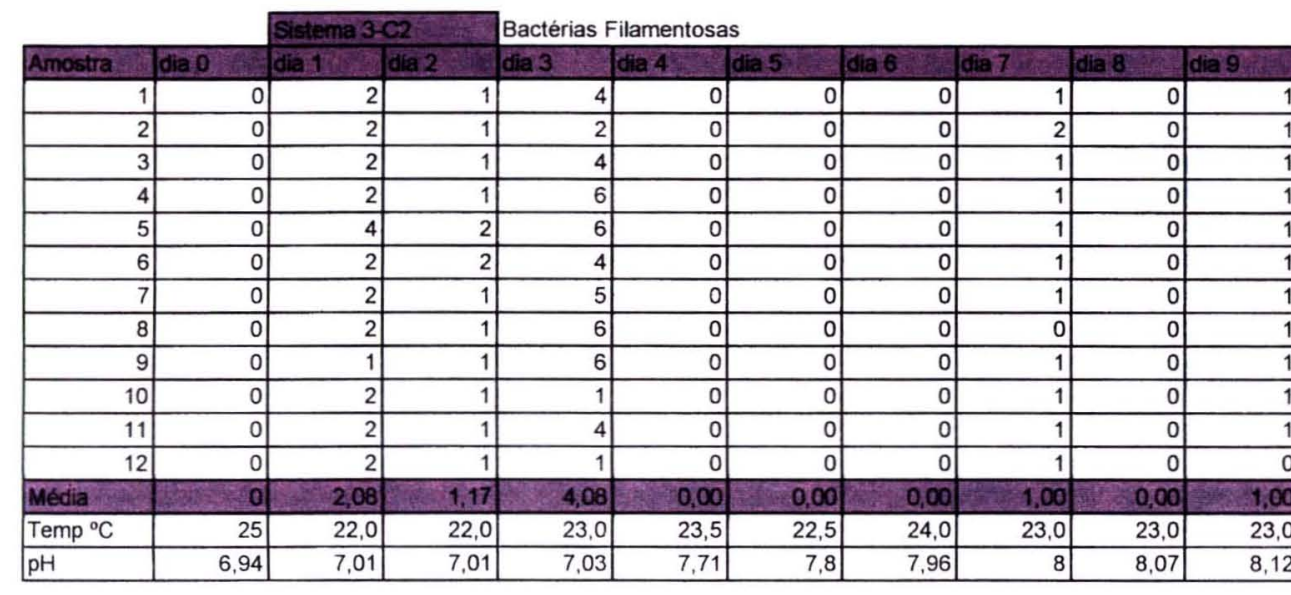

\begin{tabular}{|c|c|c|c|c|c|c|c|c|c|c|c|}
\hline & & Sistema 41 & & Bactérias F & -ilamentosa & & & & & & \\
\hline Amostra & dia 0 & dia 1 & dia 2 & dia 3 & dia 4 & dia 5. & dia 6 & dia 7 & dia 8 & dia 9 & 0 \\
\hline 1 & 0 & 1 & 1 & 6 & 0 & 0 & 0 & 1 & 0 & 0 & 1,33 \\
\hline 2 & 0 & 1 & 1 & 7 & 0 & 0 & 0 & 1 & 0 & 0 & 2,42 \\
\hline 3 & 0 & 1 & 1 & 8 & 0 & 0 & 0 & 0 & 0 & 0 & 6,00 \\
\hline 4 & 0 & 1 & 2 & 9 & 0 & 0 & 0 & 0 & 0 & 0 & 0,00 \\
\hline 5 & 0 & 2 & 2 & 10 & 0 & 0 & 0 & 0 & 0 & 0 & 0,00 \\
\hline 6 & 0 & 2 & 6 & 2 & 0 & 0 & 0 & 0 & 0 & 0 & 0,00 \\
\hline 7 & 0 & 1 & 6 & 5 & 0 & 0 & 0 & 0 & 0 & 0 & 0,17 \\
\hline 8 & 0 & 1 & 4 & 6 & 0 & 0 & 0 & 0 & 0 & 0 & 0,00 \\
\hline 9 & 0 & 3 & 2 & 13 & 0 & 0 & 0 & 0 & 0 & 0 & 0,00 \\
\hline 10 & 0 & 1 & 2 & 3 & 0 & 0 & 0 & 0 & 0 & 0 & \\
\hline 11 & 0 & 1 & 1 & 2 & 0 & 0 & 0 & 0 & 0 & 0 & \\
\hline 12 & 0 & 1 & 1 & 1 & 0 & 0 & 0 & 0 & 0 & 0 & \\
\hline Média & 0 & 1.33 & 2,42 & 6,00 & 0,00 & 0.00 & 0.00 & 0.17 & 0,00 & 0.00 & \\
\hline Temp ${ }^{\circ} \mathrm{C}$ & 25 & 22,0 & 22,0 & 23,0 & 23,5 & 22,5 & 24,0 & 23,0 & 23,0 & 23,0 & \\
\hline & 6,9 & 7,15 & 7,4 & 7,5 & 7,57 & 7,54 & 7,7 & 7,8 & 7,83 & 7,97 & \\
\hline
\end{tabular}

\begin{tabular}{|c|c|c|c|c|c|c|c|c|c|c|}
\hline & & Sistema 5 & & Bactérias $\mathrm{F}$ & Filamentosas & & & & & \\
\hline Amostra & dia 0. & dia 1 & dia 2 & dia 3 & dia 4 & dia 5 & dia 6. & dia 7 & dia 8 & dia 9 \\
\hline 1 & 0 & 1 & 1 & 2 & 0 & 0 & 0 & 0 & 요 & 2 \\
\hline 2 & 0 & 1 & 1 & 2 & 0 & 0 & 0 & 0 & 0 & 0 \\
\hline 3 & 0 & 1 & 1 & 2 & 0 & 0 & 0 & 0 & 0 & 1 \\
\hline 4 & 0 & 12 & 1 & 2 & 0 & 0 & 0 & 0 & 0 & 0 \\
\hline 5 & 0 & 2 & 1 & 2 & 0 & 1 & 0 & 0 & 0 & 2 \\
\hline 6 & 0 & 2 & 1 & 2 & 0 & 1 & 0 & 0 & 0 & 1 \\
\hline 7 & 0 & 2 & 1 & 2 & 0 & 0 & 0 & 0 & 0 & 2 \\
\hline 8 & 0 & 1 & 1 & 2 & 0 & 0 & 0 & 0 & 0 & 2 \\
\hline 9 & 0 & 1 & 1 & 2 & 0 & 0 & 0 & 0 & 0 & 2 \\
\hline 10 & 0 & 2 & 1 & 3 & 0 & 0 & 1 & 0 & 0 & 1 \\
\hline 11 & 0 & 0 & 1 & 2 & 0 & 0 & 2 & 0 & 0 & 1 \\
\hline 12 & 0 & 0 & 1 & 2 & 0 & 0 & 0 & 0 & 0 & \\
\hline Média & 0 & 2,08 & 1,00 & 2,08 & 0,00 & 0,17 & 0,25 & 0,00 & 0,00 & 1.25 \\
\hline Temp ${ }^{\circ} \mathrm{C}$ & 25 & 22,0 & 22,0 & 23,0 & 23,5 & 22,5 & 24,0 & 23,0 & 23,0 & 23,0 \\
\hline $\mathrm{pH}$ & 6,98 & 7,1 & 7,1 & 7,15 & 7,77 & 7,8 & 7,74 & 7,75 & 8,01 & 8,03 \\
\hline
\end{tabular}

\begin{tabular}{|c|c|c|c|c|c|c|c|c|c|c|c|}
\hline & & Sistema 14 & & Bactérias F & Filamentosas & & & & & & \\
\hline mostra & dia 0 & dia 1 & dia 2 & dia 3 & dia 4 , & dia 5 & lia 6 & dia 7 & dia 8 & dia 9 & 0 \\
\hline 1 & 0 & 0 & 1 & 2 & 0 & o & 0 & 0 & 1 & 이 & 1,42 \\
\hline 2 & 0 & 3 & 2 & 2 & 0 & 0 & 0 & 0 & 0 & 0 & 3,33 \\
\hline 3 & 0 & 0 & 6 & 2 & 0 & 0 & 0 & 0 & 0 & 0 & 5,00 \\
\hline 4 & 0 & 0 & 6 & 2 & 0 & 可 & 0 & 0 & 0 & 0 & 0,00 \\
\hline 5 & 0 & 4 & 4 & 7 & 0 & 0 & 0 & 0 & 0 & 0 & 0,00 \\
\hline 6 & 0 & 2 & 2 & 8 & 의 & 의 & 0 & 2 & 의 & 0 & 0,00 \\
\hline 7 & 0 & 1 & 2 & 9 & 이 & 이 & 0 & 1 & 1 & 0 & 0,25 \\
\hline 8 & 0 & 2 & 2 & 10 & 0 & 0 & 0 & 0 & 0 & 0 & 0,50 \\
\hline 9 & 0 & 2 & 6 & 12 & 0 & of & 0 & 0 & 2 & 0 & 0,00 \\
\hline 10 & 0 & 1 & 7 & 2 & 0 & of & 0 & 0 & 0 & 0 & \\
\hline 11 & 0 & 1 & 2 & 2 & 0 & 0 & 0 & 0 & 1 & 0 & \\
\hline 12 & 0 & 1 & 0 & 2 & 0 & 0 & 0 & 0 & 1 & 0 & \\
\hline Edia & & 1,42 & 3,33 & 5,00 & 0,00 & 0,00 & 0.00 & 0,25 & 0,50 & 0,00 & \\
\hline Temp ${ }^{\circ} \mathrm{C}$ & 25 & 22,0 & 22,0 & 23,0 & 23,5 & 22,5 & 24,0 & 23,0 & 23,0 & 23,0 & \\
\hline $\mathrm{pH}$ & 7,1 & 7 & 6,95 & 7 & 7,04 & 7,13 & 7,96 & 7,92 & 8 & 8 & \\
\hline
\end{tabular}




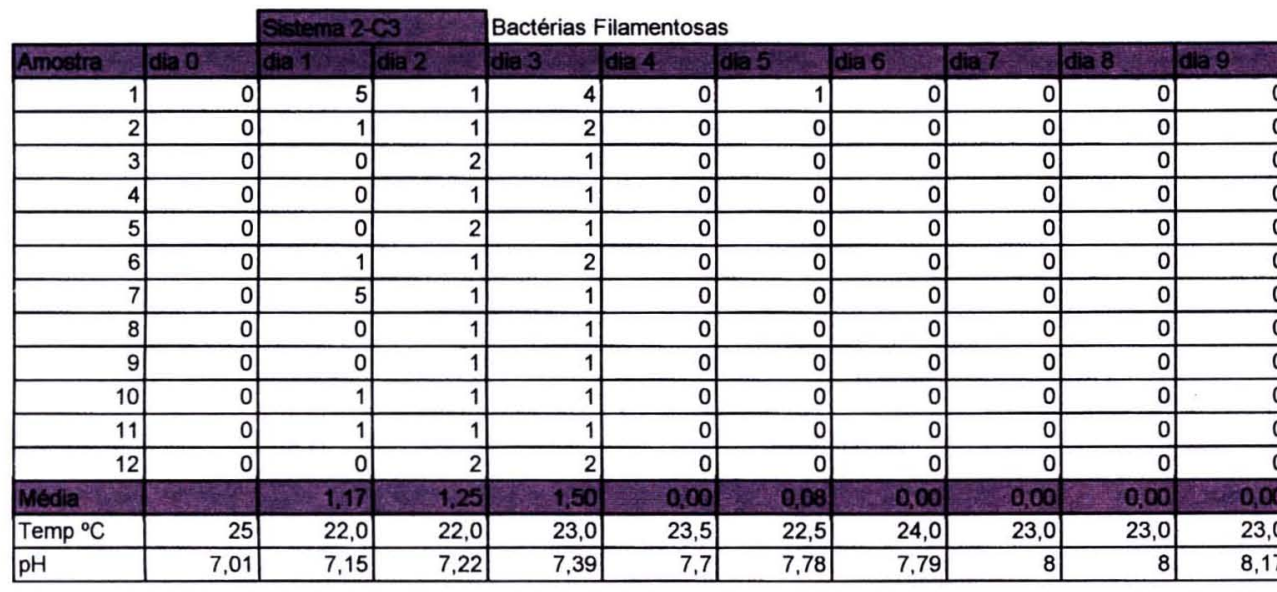

0

1,17

1,50

0,00

0,08

0,00

0,00

0,00

0,00

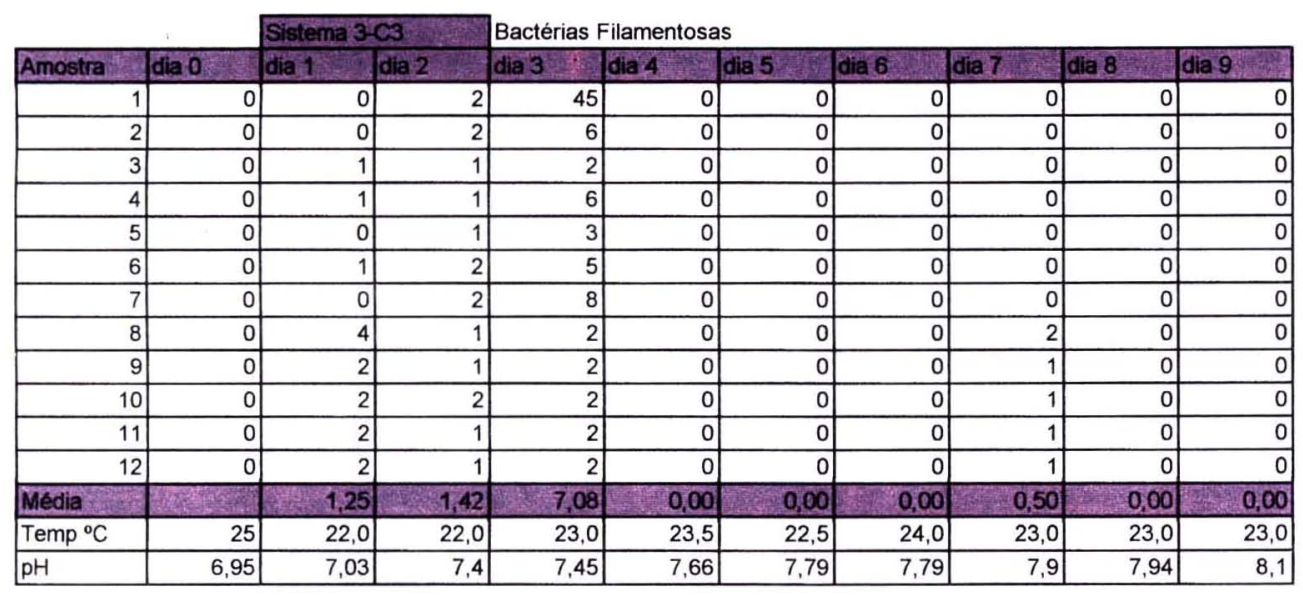

\begin{tabular}{|c|c|c|c|c|c|c|c|c|c|c|}
\hline & & Sistema 4 & C3 & Bactérias F & Filamentosa & & & & & \\
\hline Amostra & dia 0 & dia 1 & dia 2 & dia 3 & dia 4 & dia 5 & dia 6 & dia 7 & dia 8 & dia 9 \\
\hline 1 & 0 & 0 & 2 & 4 & 0 & 0 & 0 & 0 & 0 & 0 \\
\hline 2 & 0 & 1 & 2 & 2 & 0 & 0 & 0 & 0 & 0 & 0 \\
\hline 3 & 0 & 1 & 12 & 2 & 0 & 0 & 0 & 0 & 0 & 0 \\
\hline 4 & 0 & 1 & 1 & 2 & 0 & 0 & 0 & 0 & 0 & 0 \\
\hline 5 & 0 & 1 & 2 & 2 & 0 & 0 & 0 & 0 & 0 & 0 \\
\hline 6 & 0 & 1 & 3 & 2 & 0 & 0 & 0 & 0 & 0 & 0 \\
\hline 7 & 0 & 1 & 5 & 3 & 0 & 0 & 0 & 0 & 0 & 0 \\
\hline 8 & 0 & 1 & 4 & 2 & 0 & 1 & 0 & 0 & 0 & 0 \\
\hline 9 & 0 & 1 & 5 & 2 & 0 & 1 & 0 & 0 & 0 & 0 \\
\hline 10 & 0 & 1 & 6 & 3 & 0 & 0 & 0 & 0 & 0 & 0 \\
\hline 11 & 0 & 0 & 3 & 12 & 0 & 0 & 0 & 0 & 0 & 0 \\
\hline 12 & 0 & 2 & 6 & 1 & 0 & 0 & 0 & 0 & 0 & 0 \\
\hline Média & 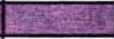 & 0,92 & 4,25 & 3,08 & 0,00 & 0,17 & 0,00 & 0,00 & 0.00 & 0,00 \\
\hline $\operatorname{Temp}^{\circ} \mathrm{C}$ & 25 & 22,0 & 22,0 & 23,0 & 23,5 & 22,5 & 24,0 & 23,0 & 23,0 & 23,0 \\
\hline $\mathrm{pH}$ & 6,87 & 7,12 & 7,36 & 7,42 & 7,68 & 7,84 & 7,78 & 7,8 & 7,84 & 8.09 \\
\hline
\end{tabular}

\begin{tabular}{|c|c|c|c|c|c|c|c|c|c|c|}
\hline & & Sistema $5-$ & C3 & Bactérias F & ilamentosa & & & & & \\
\hline Amostra & dia 0 & dia 1 & dia 2 & dia 3 & dia 4 & dia 5 & $\operatorname{dia} 6$ & dia 7 & dia 8 & dia 9 \\
\hline 1 & 0 & 0 & 0 & 1 & 0 & 0 & 0 & 1 & 0 & 0 \\
\hline 2 & 0 & 0 & 0 & 0 & 0 & 0 & 0 & 1 & 0 & 0 \\
\hline 3 & 0 & 0 & 0 & 0 & 0 & 0 & 0 & 0 & 0 & 0 \\
\hline 4 & 0 & 0 & 0 & 0 & 0 & 0 & 0 & 0 & 0 & 0 \\
\hline 5 & 0 & 0 & 0 & 0 & 0 & 1 & 0 & 0 & 0 & 0 \\
\hline 6 & 0 & 1 & 0 & 0 & 0 & 0 & 0 & 1 & 0 & 0 \\
\hline 7 & 0 & 2 & 0 & 0 & 0 & 0 & 0 & 1 & 0 & 0 \\
\hline 8 & 0 & 2 & 0 & 0 & 이 & 이 & 0 & 이 & 0 & 0 \\
\hline 9 & 0 & 1 & 0 & 0 & 0 & 0 & 0 & 0 & 0 & 0 \\
\hline 10 & 0 & 0 & 0 & 0 & 0 & 0 & 0 & 1 & 0 & 0 \\
\hline 11 & 0 & 0 & 0 & 0 & 0 & 0 & 0 & 0 & 0 & 0 \\
\hline 12 & 0 & 0 & 0 & 0 & 0 & 0 & 0 & 이 & 0 & 0 \\
\hline Média & 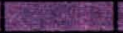 & 0,50 & 0,00 & 0,08 & 0,00 & 0,08 & 0,00 & 0,42 & 0,00 & 0,00 \\
\hline $\operatorname{Temp}^{\circ} \mathrm{C}$ & 25 & 22,0 & 22,0 & 23,0 & 23,5 & 22,5 & 24,0 & 23,0 & 23,0 & 23,0 \\
\hline $\mathrm{pH}$ & 6,9 & 7,19 & 7,35 & 7,53 & 7,64 & 7,69 & 7,78 & 7,8 & \begin{tabular}{l|l|}
7,83 \\
\end{tabular} & 8 \\
\hline
\end{tabular}


Experimento II

\begin{tabular}{|c|c|c|c|c|c|c|c|c|c|c|}
\hline & & Sistema 1 & & Ciliados & & & & & & \\
\hline Amostra & dia 0 & dia 1 & dia? & dia 3 & dia 4 & dia 5 & dia 6 & dia 7 & dia 8 & dia 9 \\
\hline 1 & 0 & 0 & 0 & 11 & 875 & 525 & 258 & 155 & 77 & 36 \\
\hline 2 & 0 & 0 & 0 & 7 & 893 & 456 & 315 & 166 & 73 & 35 \\
\hline 3 & 0 & 0 & 0 & 6 & 985 & 475 & 263 & 147 & 81 & 31 \\
\hline 4 & 0 & 0 & 0 & 5 & 965 & 524 & 274 & 131 & 79 & 44 \\
\hline 5 & 0 & 0 & 0 & 5 & 1005 & 456 & 286 & 177 & 70 & 47 \\
\hline 6 & 0 & 0 & 0 & 6 & 955 & 463 & 302 & 139 & 76 & 42 \\
\hline 7 & 0 & 0 & 0 & 6 & 932 & 456 & 295 & 141 & 82 & 45 \\
\hline 8 & 0 & 0 & 0 & 12 & 1012 & 447 & 298 & 139 & 80 & 29 \\
\hline 9 & 0 & 0 & 0 & 11 & 1017 & 525 & 305 & 157 & 75 & 39 \\
\hline 10 & 0 & 0 & 0 & 6 & 1010 & 535 & 347 & 179 & 66 & 37 \\
\hline 11 & 0 & 0 & 0 & 8 & 897 & 544 & 256 & 165 & 62 & 39 \\
\hline 12 & 0 & 0 & 0 & 14 & 977 & 526 & 347 & 167 & 67 & 46 \\
\hline Média & 0 & 0,00 & 0,00 & 8,08 & 960.25 & 494,33 & 295,50 & 155,25 & 74,00 & 39,17 \\
\hline Temp ${ }^{\circ} \mathrm{C}$ & 25 & 22,0 & 22,0 & 22,0 & 23,5 & 22,5 & 24,0 & 23,0 & 23,0 & 23,0 \\
\hline $\mathrm{pH}$ & 7,01 & 7,15 & 7,23 & 7,37 & 7,55 & 7,62 & 7.77 & 7,8 & 7,89 & 7,9 \\
\hline
\end{tabular}

\begin{tabular}{|c|c|c|c|c|c|c|c|c|c|c|c|}
\hline Amostra & dia 0 & dia 1 & dia 2 & dia 3 & dia 4 & dia 5. & dia 6 . & dia 7 & dia 8 & dia 9 & \\
\hline 1 & 0 & 0 & 0 & 27 & 1015 & 724 & \begin{tabular}{|r|}
346 \\
\end{tabular} & \begin{tabular}{|r|}
186 \\
\end{tabular} & 95 & 49 & 0,00 \\
\hline 2 & 0 & 0 & 0 & 27 & 1236 & 756 & 366 & 195 & 88 & 58 & 0,00 \\
\hline 3 & 0 & 0 & 0 & 33 & 1366 & 744 & 414 & 174 & 92 & 45 & 35,92 \\
\hline 4 & 0 & 0 & 0 & 37 & 1256 & 752 & 374 & 166 & 102 & 39 & 1236,83 \\
\hline 5 & 0 & 0 & 0 & 35 & 1315 & 612 & 396 & 192 & 86 & 44 & 736,67 \\
\hline 6 & 0 & 0 & 0 & 32 & 1317 & 762 & 414 & 152 & 92 & 47 & 362,00 \\
\hline 7 & 0 & 0 & 0 & 39 & 1258 & 695 & 425 & 211 & 94 & 46 & 191,67 \\
\hline 8 & 0 & 0 & 0 & 42 & 1256 & 751 & 355 & 214 & 74 & 57 & 87,75 \\
\hline 9 & 0 & 0 & 0 & 44 & 1258 & 765 & 312 & 223 & 75 & 51 & 46,58 \\
\hline 10 & 0 & 0 & 0 & 21 & 1315 & 788 & 325 & 217 & 79 & 35 & \\
\hline 11 & 0 & 0 & 0 & 37 & 1254 & 725 & 302 & 196 & 86 & 41 & \\
\hline 12 & 0 & 0 & 0 & 57 & 996 & 766 & 315 & 174 & 90 & 47 & \\
\hline Média & 0 & 0,00 & 0.00 & 35,92 & 1236,83 & 736,67 & 362,00 & 191,67 & 87,75 & 46.58 & \\
\hline Temp ${ }^{\circ} \mathrm{C}$ & 25 & 22,0 & 22,0 & 22,0 & 23,5 & 22,5 & 24,0 & 23,0 & 23,0 & 23,0 & \\
\hline $\mathrm{pH}$ & 6,95 & 7,1 & 7,18 & 7,22 & 7,23 & 7,06 & 7,52 & 7,7 & 7,78 & 7,9 & \\
\hline
\end{tabular}

\begin{tabular}{|c|c|c|c|c|c|c|c|c|c|c|}
\hline & & Sistema 3 & & Ciliados & & & & & & \\
\hline Amostra & dia 0 & dia 1 & dia 2 & dia 3 & dia 4 & dia 5 & dia 6 & dia 7 & dia 8 & dia 9 \\
\hline 1 & 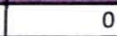 & 0 & 0 & 0 & 658 & 362 & 225 & 124 & 64 & 7 \\
\hline 2 & 0 & 0 & 0 & 0 & 623 & 370 & 274 & 132 & 67 & 4 \\
\hline 3 & 0 & 0 & 0 & 0 & 653 & 356 & 265 & 115 & 44 & 9 \\
\hline 4 & 0 & 0 & 0 & 0 & 670 & 345 & 226 & 115 & 51 & 9 \\
\hline 5 & 0 & 0 & 0 & 0 & 652 & 372 & 206 & 136 & 44 & 11 \\
\hline 6 & 0 & 0 & 0 & 0 & 714 & 352 & 256 & 135 & 49 & 6 \\
\hline 7 & 0 & 0 & 0 & 0 & 772 & 354 & 267 & 125 & 41 & 5 \\
\hline 8 & 0 & 0 & 0 & 0 & 724 & 423 & 295 & 145 & 66 & 5 \\
\hline 9 & 0 & 0 & 0 & 0 & 736 & 369 & 266 & 132 & 53 & 6 \\
\hline 10 & 0 & 0 & 0 & 0 & 668 & 335 & 324 & 133 & 42 & 12 \\
\hline 11 & 0 & 0 & 0 & 0 & 645 & 315 & 336 & 149 & 37 & 8 \\
\hline 12 & 0 & 0 & 0 & 0 & 695 & 325 & 314 & 159 & 41 & 9 \\
\hline Média & 0 & 0,00 & 0,00 & 0,00 & 684,17 & 356,50 & 271,17 & 133,33 & 49,92 & 7,58 \\
\hline Temp ${ }^{\circ} \mathrm{C}$ & 25,0 & 22 & 22,0 & 22,0 & 23,5 & 22,5 & 24,0 & 23,0 & 23,0 & 23,0 \\
\hline $\mathrm{pH}$ & 6,95 & 7,01 & 7 & 6,9 & 6,81 & 7,81 & 8,04 & 8,1 & 8,21 & 8,23 \\
\hline
\end{tabular}

\begin{tabular}{|c|c|c|c|c|c|c|c|c|c|c|}
\hline & & Sistema 4 & & Ciliados & & & & & & \\
\hline Amostra & dia 0 & dia 1 & dia 2 & dia 3 & dia 4 & dia 5 & dia 6 & dia 7 & dia 8 & dia 9 \\
\hline 1 & 0 & 0 & 0 & 117 & 1152 & 705 & 330 & 265 & 102 & 56 \\
\hline 2 & 0 & 0 & 0 & 92 & 1252 & 721 & 400 & 254 & 95 & 62 \\
\hline 3 & 0 & 0 & 0 & 75 & 1163 & 714 & 400 & 263 & 112 & 55 \\
\hline 4 & 0 & 0 & 0 & 112 & 1162 & 692 & 440 & 278 & 121 & 60 \\
\hline 5 & 0 & 0 & 0 & 85 & 1266 & 793 & 390 & 270 & 117 & 62 \\
\hline 6 & 0 & 0 & 0 & 72 & 1056 & 752 & 400 & 272 & 112 & 71 \\
\hline 7 & 0 & 0 & 0 & 77 & 1145 & 744 & 460 & 255 & 92 & 52 \\
\hline 8 & 0 & 0 & 0 & 89 & 1063 & 722 & 460 & 236 & 121 & 56 \\
\hline 9 & 0 & 0 & 0 & 57 & 1057 & 705 & 480 & 268 & 89 & 60 \\
\hline 10 & 0 & 0 & 0 & 74 & 1136 & 690 & 450 & 295 & 96 & 63 \\
\hline 11 & 0 & 0 & 0 & 77 & 1170 & 692 & 480 & 278 & 102 & 55 \\
\hline 12 & 0 & 0 & 0 & 102 & 1215 & 684 & 450 & 269 & 122 & 56 \\
\hline Média & 0 & 0,00 & 0,00 & 85,75 & 1153,08 & 717,83 & 428,33 & 266,92 & 106,75 & 59,00 \\
\hline Temp ${ }^{\circ} \mathrm{C}$ & 25,0 & 22 & 22,0 & 22,0 & 23,5 & 22,5 & 24,0 & 23,0 & 23,0 & 23,0 \\
\hline $\mathrm{pH}$ & 6,87 & 6,9 & 7,15 & 7,55 & 7,85 & 7,88 & 8,03 & 8,1 & 8,16 & 8,2 \\
\hline
\end{tabular}


Ciliados

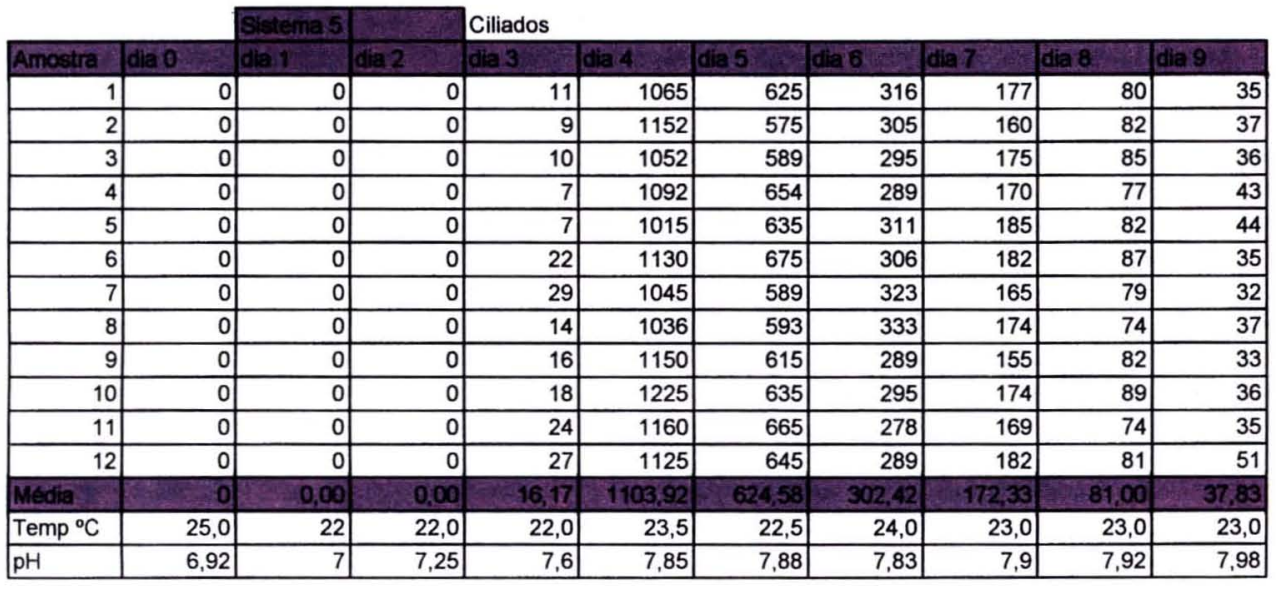

\begin{tabular}{|c|c|c|c|c|c|c|c|c|c|c|}
\hline & & Sistema 1- & & Ciliados & & & & & & \\
\hline Amostra & dia 0 & dia 1 & dia 2 & dia 3 & dia 4 & dia 5 . & dia 6 & dia 7 & dia 8 & dia 9 \\
\hline \begin{tabular}{r|}
1 \\
\end{tabular} & 0 & 0 & 0 & 4 & 714 & 349 & 56 & $\begin{array}{r}17 \\
\end{array}$ & 13 & 20 \\
\hline 2 & 0 & 0 & 0 & 13 & 725 & 325 & 65 & 23 & 12 & 23 \\
\hline 3 & 0 & 0 & 0 & 3 & 745 & 362 & 140 & 19 & 10 & 25 \\
\hline 4 & 0 & 0 & 0 & 3 & 765 & 392 & 100 & 20 & 10 & 28 \\
\hline 5 & 0 & 0 & 0 & 6 & 812 & 295 & 150 & 21 & 9 & 22 \\
\hline 6 & 0 & 0 & 0 & 5 & 685 & 302 & 150 & 19 & 9 & 24 \\
\hline 7 & 0 & 0 & 0 & 4 & 742 & 332 & 160 & 19 & 9 & 20 \\
\hline 8 & 0 & 0 & 0 & 3 & 754 & 321 & 180 & 18 & 14 & 22 \\
\hline 9 & 0 & 0 & 0 & 3 & 755 & 321 & 120 & 19 & 10 & 26 \\
\hline 10 & 0 & 0 & 0 & 6 & 685 & 314 & 150 & 21 & 7 & 29 \\
\hline 11 & 0 & 0 & 0 & 6 & 630 & 318 & 150 & 19 & 5 & 26 \\
\hline 12 & 0 & 0 & 0 & 4 & 615 & 315 & 150 & 19 & 5 & 23 \\
\hline Média & 0 & 0,00 & 0,00 & 5,00 & 718,92 & 328,83 & 130,92 & 19,50 & 9,42 & 24,00 \\
\hline Temp ${ }^{\circ} \mathrm{C}$ & 25,0 & 22,0 & 22,0 & 23,0 & 23,5 & 22,5 & 24,0 & 24,0 & 23,0 & 23,0 \\
\hline $\mathrm{pH}$ & 7,05 & 7,2 & 7,2 & 7,25 & 7,79 & 7,76 & 7,92 & 7,77 & 7.9 & \\
\hline
\end{tabular}

\begin{tabular}{|c|c|c|c|c|c|c|c|c|c|c|}
\hline & & Sistema 2 & & Ciliados & & & & & & \\
\hline Amostra & dia 0 & dia 1 & $\longdiv { \text { dia } 2 }$ & dia 3 & dia 4 & dia 5 & dia 6 & dia 7 & dia 8 & dia 9 \\
\hline 1 & 0 & 0 & 0 & 26 & 844 & 365 & 175 & 93 & 67 & 55 \\
\hline 2 & 0 & 0 & 0 & 30 & 866 & 389 & 169 & 106 & 61 & 29 \\
\hline 3 & 0 & 0 & 0 & 28 & 892 & 415 & 185 & 104 & 74 & 41 \\
\hline 4 & 0 & 0 & 0 & 27 & 815 & 396 & 179 & 92 & 61 & 39 \\
\hline 5 & 0 & 0 & 0 & 26 & 912 & 425 & 169 & 96 & 51 & 40 \\
\hline 6 & 0 & 0 & 0 & 25 & 865 & 392 & 165 & 114 & 84 & 41 \\
\hline 7 & 0 & 0 & 0 & 26 & 914 & 366 & 158 & 123 & 79 & 35 \\
\hline 8 & 0 & 0 & 0 & 27 & 965 & 378 & 179 & 115 & 61 & 40 \\
\hline 9 & 0 & 0 & 0 & 30 & 947 & 395 & 187 & 86 & 57 & 41 \\
\hline 10 & 0 & 0 & 0 & 30 & 925 & 425 & 211 & 92 & 54 & 36 \\
\hline 11 & 0 & 0 & 0 & 24 & 899 & 445 & 236 & 95 & 67 & 44 \\
\hline 12 & 0 & 0 & 0 & 22 & 923 & 426 & 215 & 91 & 77 & 51 \\
\hline Média & 0 & 0,00 & 0,00 & 26,75 & 897,25 & 401,42 & 185,67 & 100,58 & 66,08 & 41,00 \\
\hline Temp ${ }^{\circ} \mathrm{C}$ & 25,0 & 22,0 & 22,0 & 23,0 & 23,5 & 22,5 & 24,0 & 23,0 & 23,0 & 23,0 \\
\hline $\mathrm{pH}$ & 6,96 & 7 & 7 & 7,02 & 7,48 & 7,66 & 7,91 & 8 & 8,02 & 8 \\
\hline
\end{tabular}

\begin{tabular}{|c|c|c|c|c|c|c|c|c|c|c|}
\hline & & Sistema 3 & & Ciliados & & & & & & \\
\hline Amostra & dia 0 & dia 1 & dia 2 & dia 3 & dia 4 & dia 5 & dia 6 & dia 7 & dia 8 & dia 9 \\
\hline 1 & 0 & 0 & 0 & 3 & 895 & 412 & 256 & 144 & 182 & 174 \\
\hline 2 & 0 & 0 & 0 & 5 & 885 & 379 & 245 & 166 & 189 & 192 \\
\hline 3 & 0 & 0 & 0 & 2 & 1015 & 405 & 266 & 174 & 174 & 204 \\
\hline 4 & 이 & 0 & 0 & 3 & 1125 & 381 & 225 & 144 & 162 & 190 \\
\hline 5 & 0 & 0 & 0 & 2 & 1065 & 377 & 215 & 139 & 192 & 210 \\
\hline 6 & 0 & 0 & 0 & 5 & 1152 & 365 & 315 & 159 & 174 & 202 \\
\hline 7 & 0 & 0 & 0 & 3 & 1145 & 374 & 182 & 173 & 212 & 176 \\
\hline 8 & 0 & 0 & 0 & 2 & 1074 & 395 & 293 & 165 & 204 & 174 \\
\hline 9 & 0 & 0 & 0 & 3 & 1065 & 365 & 270 & 184 & 214 & 178 \\
\hline 10 & 0 & 0 & 0 & 3 & 1150 & 394 & 262 & 177 & 202 & 169 \\
\hline 11 & 0 & 0 & 0 & 3 & 1215 & 374 & 285 & 192 & 212 & 215 \\
\hline 12 & 이 & 0 & 0 & 3 & 1165 & 385 & 311 & 145 & 174 & 219 \\
\hline Média & 0 & 0,00 & 0,00 & 3,08 & 1079,25 & 383,83 & 260,42 & 163,50 & 190,92 & 191,92 \\
\hline Temp $^{\circ} \mathrm{C}$ & 25,0 & 22,0 & 23,0 & 23,0 & 23,5 & 22,5 & 24,0 & 23,0 & 23,0 & 23,0 \\
\hline $\mathrm{pH}$ & 6,95 & 7,25 & 7,35 & 7,59 & 7,7 & 7,73 & 8,03 & 8 & 8,02 & 8,04 \\
\hline
\end{tabular}




\begin{tabular}{|c|c|c|c|c|c|c|c|c|c|c|}
\hline \multirow{2}{*}{\begin{tabular}{|l|l} 
Amostra & di:
\end{tabular}} & & \multicolumn{2}{|c|}{ Sistema 4-C1 } & \multicolumn{7}{|l|}{ Ciliados } \\
\hline & dia 0. & dia 1 & dia 2 & dia 3 & dia 4 & dia 5 & dia 6 & dia 7. & dia 8 & dia 9 \\
\hline 1 & 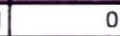 & 0 & 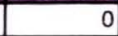 & 2 & 912 & 390 & 120 & 210 & 31 & \\
\hline 2 & $\overline{0}$ & 0 & 0 & 2 & 900 & 347 & 130 & 180 & 27 & \\
\hline 3 & 0 & 0 & 0 & 5 & 922 & 370 & 140 & 200 & 32 & \\
\hline 4 & 0 & 0 & 0 & 6 & 945 & 330 & 166 & 190 & 26 & \\
\hline 5 & 0 & 0 & 0 & 3 & 932 & 380 & 172 & 195 & 24 & \\
\hline 6 & 0 & 0 & 0 & 5 & 962 & 390 & 222 & 200 & 29 & \\
\hline 7 & 0 & 0 & 0 & 8 & 963 & 405 & 146 & 210 & 30 & \\
\hline 8 & 0 & 0 & 0 & 9 & 1105 & 360 & 132 & 205 & 22 & \\
\hline 9 & 0 & 0 & 0 & 6 & 1123 & 360 & 122 & 200 & 21 & \\
\hline 10 & 0 & 0 & 0 & 3 & 994 & 330 & 155 & 190 & 20 & \\
\hline 11 & 0 & 0 & 0 & 5 & 974 & 405 & 146 & 196 & 37 & \\
\hline 12 & 0 & 0 & 0 & 6 & 966 & 410 & 160 & 180 & 24 & \\
\hline Média & 0 & 0,00 & 0,00 & 5,00 & 974,83 & 373,08 & 150,92 & 196,33 & 26,92 & 5,00 \\
\hline Temp ${ }^{\circ} \mathrm{C}$ & 25,0 & 22,0 & 22,0 & 23,0 & 23,5 & 22,5 & 24,0 & 23,0 & 23,0 & 23,0 \\
\hline $\mathrm{pH}$ & 6,9 & 7,27 & 7,3 & 7,58 & 7.63 & 7,89 & 8,04 & 8,06 & 8,11 & 8.19 \\
\hline
\end{tabular}

\begin{tabular}{|c|c|c|c|c|c|c|c|c|c|c|}
\hline & & Sistema 5- & $C 1$ & Ciliados & & & & & & \\
\hline Amostra & dia 0 & dia 1 & dia 2 & dia 3 & dia 4 & dia 5. & dia 6 & dia 7 & dia 8 & dia 9 \\
\hline 1 & 0 & 0 & 0 & 1 & 900 & 360 & 125 & 312 & 74 & 11 \\
\hline 2 & 0 & 0 & 0 & 1 & 950 & 390 & 122 & 342 & 74 & 9 \\
\hline 3 & 0 & 0 & 0 & 1 & 960 & 350 & 125 & 312 & 66 & 2 \\
\hline 4 & 0 & 0 & 0 & 1 & 900 & 350 & 133 & 325 & 83 & 4 \\
\hline 5 & 0 & 0 & 0 & 5 & 900 & 360 & 142 & 319 & 74 & 11 \\
\hline 6 & 0 & 0 & 0 & 6 & 900 & 360 & 152 & 298 & 79 & 18 \\
\hline 7 & 0 & 0 & 0 & 5 & 900 & 370 & 132 & 300 & 75 & 5 \\
\hline 8 & 0 & 0 & 0 & 6 & 900 & 330 & 141 & 306 & 69 & 6 \\
\hline 9 & 0 & 0 & 0 & 5 & 900 & 325 & 123 & 311 & 89 & 7 \\
\hline 10 & 0 & 0 & 0 & 6 & 952 & 330 & 114 & 292 & 82 & 2 \\
\hline 11 & 0 & 0 & 0 & 5 & 940 & 360 & 105 & 316 & 79 & 2 \\
\hline 12 & 0 & 0 & 0 & 7 & 900 & 370 & 112 & 290 & 92 & 5 \\
\hline Media & 0 & 0,00 & 0,00 & 4,08 & 916,83 & 354,58 & 127.17 & 310,25 & 78,00 & 6,83 \\
\hline Temp ${ }^{\circ} \mathrm{C}$ & 25,0 & 22,0 & 22,0 & 23,0 & 23,5 & 22,5 & 23 & 24,0 & 23,0 & 23,0 \\
\hline $\mathrm{pH}$ & 7,1 & 7,5 & 7,67 & 7,97 & 7,73 & 7,75 & 7,97 & 8,1 & 7,83 & 8,1 \\
\hline
\end{tabular}

Quantidade microrganismos/substrato DBO $350 \mathrm{mg} / \mathrm{laeraço} \mathrm{continua/} 1,56 \mathrm{pppm}$ Cobre no 4 dia.

\begin{tabular}{|c|c|c|c|c|c|c|c|c|c|c|c|}
\hline & \multicolumn{2}{|c|}{ Sistema $1 \mathrm{C}_{2}$} & \multirow{2}{*}{\multicolumn{7}{|c|}{ Ciliados }} & \\
\hline & & & & & & & & & & & \\
\hline Amostra & dia 0 & dia 1 & alaz & dia 3 & dia 4 & dia 5 & jaa 6. & dia 7 & dia 8 & dia 9 & 0 \\
\hline 1 & 0 & 0 & 0 & 0 & 770 & 190 & 50 & 251 & 62 & 5 & 0,00 \\
\hline 2 & 0 & 0 & 0 & 0 & 765 & 190 & 60 & 235 & 52 & 8 & 0,00 \\
\hline 3 & 0 & 0 & 0 & 0 & 812 & 195 & 65 & 238 & 51 & 15 & 0,00 \\
\hline 4 & 0 & 0 & 0 & 0 & 845 & 190 & 45 & 247 & 45 & 10 & 788,33 \\
\hline 5 & 0 & 0 & 0 & 0 & 779 & 190 & 60 & 244 & 74 & 11 & 193,83 \\
\hline 6 & 0 & 0 & 0 & 0 & 852 & 190 & 50 & 211 & 78 & 10 & 53,25 \\
\hline 7 & 0 & 0 & 0 & 0 & 763 & 190 & 50 & 217 & 86 & 9 & 228,00 \\
\hline 8 & 0 & 0 & 0 & 0 & 889 & 201 & 54 & 208 & 77 & 8 & 64,67 \\
\hline 9 & 0 & 0 & 0 & 0 & 812 & 200 & 55 & 216 & 85 & 10 & 9,17 \\
\hline 10 & 0 & 0 & 0 & 0 & 714 & 210 & 50 & 225 & 47 & 3 & \\
\hline 11 & 0 & 0 & 0 & 0 & 727 & 220 & 60 & 226 & 51 & 9 & \\
\hline 12 & 0 & 0 & 0 & 0 & 732 & 160 & 40 & 218 & 68 & 12 & \\
\hline Média & 0 & 0,00 & 0,00 & 0,00 & 788,33 & 193,83 & 53,25 & 228,00 & 64,67 & 9,17 & \\
\hline Temp ${ }^{\circ} \mathrm{C}$ & 25,0 & 22,0 & 22,0 & 23,0 & 23,5 & 22,5 & 23,0 & 23,0 & 23,0 & 23,0 & \\
\hline $\mathrm{pH}$ & 6,96 & 7 & 7 & 7 & 7,78 & 7,61 & 7.85 & 7,89 & 7,9 & 8 & \\
\hline & & tema 2 & $\mathrm{C2}$ & Ciliados & & & & & & & \\
\hline Amostra: & dia 0 & dia 1 & dia 2 & dia 3 & dia 4 & dia 5 & dia 6 & dia 7 & dia 8 & dia 9 & 0 \\
\hline 1 & 0 & 0 & 0 & 0 & 1212 & 242 & 77 & 215 & 23 & 15 & 0,00 \\
\hline 2 & 0 & 0 & 0 & 0 & 1242 & 244 & 84 & 172 & 24 & 21 & 0,00 \\
\hline 3 & 0 & 0 & 0 & 0 & 1274 & 258 & 79 & 202 & 10 & 17 & 0,00 \\
\hline 4 & 0 & 0 & 0 & 0 & 1227 & 244 & 70 & 195 & 20 & 15 & 1279,58 \\
\hline 5 & 0 & 0 & 0 & 0 & 1317 & 214 & 71 & 177 & 23 & 12 & 245,92 \\
\hline 6 & 0 & 0 & 0 & 0 & 1417 & 244 & 87 & 214 & 25 & 17 & 86,50 \\
\hline 7 & 0 & 0 & 0 & 0 & 1236 & 247 & 84 & 207 & 30 & 22 & 164,50 \\
\hline 8 & 0 & 0 & 0 & 0 & 1315 & 236 & 102 & 107 & 25 & 11 & 24,58 \\
\hline 9 & 0 & 0 & 0 & 0 & 1325 & 247 & 106 & 113 & 30 & 19 & 17,67 \\
\hline 10 & 0 & 0 & 0 & 0 & 1210 & 244 & 94 & 125 & 31 & 20 & \\
\hline 11 & 0 & 0 & 0 & 0 & 1236 & 268 & 97 & 132 & 32 & 22 & \\
\hline 12 & 0 & 0 & 0 & 0 & 1344 & 263 & 87 & 115 & 22 & 21 & \\
\hline Média & $\overline{0}$ & 0,00 & 0,00 & 0,00 & 1279,58 & 245,92 & 86,50 & 164.50 & 24,58 & 17.67 & \\
\hline $\operatorname{Temp}^{\circ} \mathrm{C}$ & 25,0 & 22,0 & 22,0 & 23,0 & 23,5 & 22,5 & 24,0 & 23,0 & 23,0 & 23,0 & \\
\hline pH & 7 & 7,1 & 7,15 & 7,59 & 7,64 & 7,71 & 7,91 & 8 & 8,03 & 8,18 & \\
\hline
\end{tabular}


Sistema 3-C2 Ciliados

\begin{tabular}{|c|c|c|c|c|c|c|c|c|c|c|}
\hline Amostra & $\operatorname{dia} 0$ & dia 1 & dia 2 & dia 3 & dia 4 & dia 5 & dia 6 & $\operatorname{dia} 7$ & dia 8 & dia 9 \\
\hline 1 & 0 & 0 & 0 & 13 & 211 & 202 & 55 & 11 & 2 & 0 \\
\hline 2 & 0 & 0 & 0 & 17 & 1011 & 226 & 40 & 21 & 1 & 0 \\
\hline 3 & 0 & 0 & 0 & 14 & 1112 & 219 & 36 & 19 & 3 & 0 \\
\hline 4 & 0 & 0 & 0 & 25 & 1025 & 239 & 40 & 20 & 1 & 0 \\
\hline 5 & 0 & 0 & 0 & 16 & 1036 & 221 & 41 & 20 & 2 & 0 \\
\hline 6 & 0 & 0 & 0 & 14 & 1132 & 212 & 36 & 20 & 2 & 0 \\
\hline 7 & 0 & 0 & 0 & 23 & 1214 & 214 & 44 & 21 & 3 & 0 \\
\hline 8 & 0 & 0 & 0 & 15 & 1110 & 203 & 53 & 20 & 2 & 0 \\
\hline 9 & 0 & 0 & 0 & 16 & 1014 & 193 & 32 & 19 & 2 & 0 \\
\hline 10 & 0 & 0 & 0 & 33 & 1021 & 175 & 33 & 19 & 2 & 0 \\
\hline 11 & 0 & 0 & 0 & 17 & 1021 & 142 & 28 & 18 & 2 & 0 \\
\hline 12 & 0 & 0 & 0 & 15 & 1214 & 124 & 44 & 18 & 2 & 0 \\
\hline Média & 0 & 0,00 & 0,00 & 18,17 & 1010,08 & 197,50 & 40,17 & 18,83 & 2,00 & 0,00 \\
\hline Temp $^{\circ} \mathrm{C}$ & 25,0 & & 22,0 & 23,0 & 23,5 & 22,5 & 24,0 & 23,0 & 23,0 & 23,0 \\
\hline $\mathrm{pH}$ & 6,94 & & 7,01 & 7 & 7,71 & 7,8 & 7,96 & 8 & 8,07 & 8,12 \\
\hline
\end{tabular}

\begin{tabular}{|c|c|c|c|c|c|c|c|c|c|c|}
\hline & & $\longdiv { \text { Sistema } 4 }$ & & Ciliados & & & & & & \\
\hline Amostra: & dia 0 & dia 1 & dia 2 & dia 3 & dia 4 & dia 5 & dia 6 & dia 7 & dia 8 & dia 9 \\
\hline $\begin{array}{r}1 \\
\end{array}$ & 0 & 0 & 0 & 70 & 912 & \begin{tabular}{|r|}
157 \\
\end{tabular} & 151 & 46 & 14 & 11 \\
\hline 2 & 0 & 0 & 0 & 33 & 914 & 155 & 151 & 59 & 19 & 13 \\
\hline 3 & 0 & 0 & 0 & 39 & 877 & 162 & 141 & 60 & 15 & 11 \\
\hline 4 & 0 & 0 & 0 & 30 & 852 & 116 & 124 & 51 & 15 & 10 \\
\hline 5 & 0 & 0 & 0 & 41 & 914 & 117 & 106 & 42 & 14 & 14 \\
\hline 6 & 0 & 0 & 0 & 15 & 1057 & 174 & 144 & 42 & 18 & 11 \\
\hline 7 & 0 & 0 & 0 & 24 & 1074 & 157 & 113 & 60 & 17 & 10 \\
\hline 8 & 0 & 0 & 0 & 39 & 1157 & 177 & 124 & 55 & 12 & 10 \\
\hline 9 & 0 & 0 & 0 & 38 & 957 & 142 & 166 & 39 & 9 & 10 \\
\hline 10 & 0 & 0 & 0 & 35 & 944 & 177 & 151 & 32 & 9 & 10 \\
\hline 11 & 0 & 0 & 0 & 32 & 946 & 151 & 142 & 42 & 9 & 9 \\
\hline 12 & 0 & 0 & 0 & 30 & 947 & 141 & 135 & 36 & 7 & $\overline{12}$ \\
\hline Media & 0 & 0,00 & 0,00 & 35,50 & 962,58 & 152,17 & 137,33 & 47,00 & 13,17 & 10,92 \\
\hline Temp ${ }^{\circ} \mathrm{C}$ & 25,0 & & 22,0 & 23,0 & 23,5 & 22,5 & 24,0 & 23,0 & 23,0 & 23,0 \\
\hline pH & 6,9 & & 7,4 & 7,5 & 7,57 & 7,54 & 7,7 & 7,8 & 7,83 & 7,97 \\
\hline
\end{tabular}

\begin{tabular}{|c|c|c|c|c|c|c|c|c|c|c|}
\hline & & Sistema 5 & & Ciliados & & & & & & \\
\hline Amostra & dia 0 & dia 1 & dia 2 & dia 3 & dia 4 & dia 5 & dia 6 & dia 7 & $\operatorname{dia} 8$ & dia 9 \\
\hline 1 & 0 & 0 & 0 & 14 & 1185 & 266 & 74 & 93 & 136 & 0 \\
\hline 2 & 0 & 0 & 0 & 10 & 1057 & 235 & 71 & 113 & 117 & 0 \\
\hline 3 & 0 & 0 & 0 & 11 & 1162 & 205 & 78 & 96 & 162 & 0 \\
\hline 4 & 0 & 0 & 0 & 17 & 1272 & 212 & 77 & 90 & 142 & 0 \\
\hline 5 & 0 & 0 & 0 & 22 & 1252 & 190 & 82 & 107 & 164 & 0 \\
\hline 6 & 0 & 0 & 0 & 21 & 1142 & 220 & 70 & 92 & 135 & 0 \\
\hline 7 & 0 & 0 & 0 & 9 & 1014 & 222 & 73 & 105 & 119 & 0 \\
\hline 8 & 0 & 0 & 0 & 11 & 1190 & 230 & 77 & 85 & 112 & 0 \\
\hline 9 & 0 & 0 & 0 & 14 & 1311 & 245 & 64 & 75 & 117 & 0 \\
\hline 10 & 0 & 0 & 0 & 18 & 1152 & 230 & 72 & 92 & 135 & 0 \\
\hline 11 & 0 & 0 & 可 & 19 & 1102 & 229 & 82 & 85 & 147 & 0 \\
\hline 12 & 0 & 0 & 0 & 22 & 1112 & 260 & 78 & 96 & 125 & 0 \\
\hline Média & 0 & 0,00 & 0,00 & 15,67 & 1162,58 & 228,67 & 74,83 & 94,08 & 134,25 & 0,00 \\
\hline $\operatorname{Temp}^{\circ} \mathrm{C}$ & 25,0 & 22,0 & 22,0 & 23,0 & 23,50 & 22,5 & 24,0 & 23,0 & 23,0 & 23,0 \\
\hline pH & 6,98 & 7,1 & 7,1 & 7,15 & 7,77 & 7,8 & 7,74 & 7,75 & 8,01 & 8,03 \\
\hline
\end{tabular}

\begin{tabular}{|c|c|c|c|c|c|c|c|c|c|c|c|}
\hline & & Sistema 1- & & Ciliados & & & & & & & \\
\hline Amostra & dia 0 & dia 1 & dia 2 & dia 3 & dia 4 & dia 5 & dia 6 & dia 7 & dia 8 & dia 9 & \\
\hline 1 & 0 & 0 & 0 & 0 & $\begin{array}{r}745 \\
\end{array}$ & 62 & 10 & 0 & 0 & 이 & 0,00 \\
\hline 2 & 0 & 0 & 0 & 0 & 776 & 69 & 15 & 0 & 0 & 0 & 0,00 \\
\hline 3 & 0 & 0 & 0 & 0 & 725 & 66 & 20 & 0 & 0 & 0 & 0,00 \\
\hline 4 & 0 & 0 & 0 & 0 & 816 & 51 & 16 & 0 & 0 & 0 & 784,08 \\
\hline 5 & 0 & 0 & 0 & 0 & 932 & 66 & 11 & 0 & 0 & 0 & 59,08 \\
\hline 6 & 0 & 의 & 0 & 0 & 813 & 66 & 15 & 의 & 요 & 0 & 13,25 \\
\hline 7 & 0 & 0 & 0 & 0 & 834 & 55 & 21 & 요 & 0 & 0 & 0,00 \\
\hline 8 & 0 & 요 & 0 & 0 & 824 & 52 & 10 & 요 & 0 & 의 & 0,00 \\
\hline 9 & 0 & 0 & 0 & 0 & 789 & 49 & 16 & 0 & 0 & 0 & 0,00 \\
\hline 10 & 0 & 의 & $\underline{0}$ & 의 & 754 & 65 & 12 & 요 & 0 & 0 & \\
\hline 11 & 0 & 0 & 0 & 0 & 732 & 59 & 13 & 0 & 0 & 0 & \\
\hline 12 & 0 & 0 & 0 & 0 & 669 & 49 & 0 & 0 & 0 & 0 & \\
\hline Média & & 0,00 & 0,00 & 0,00 & 784,08 & 59,08 & 13,25 & 0,00 & 0,00 & 0,00 & \\
\hline Temp ${ }^{\circ} \mathrm{C}$ & 25,0 & 20,0 & 21,0 & 23,0 & 23,5 & 22,5 & 24,0 & 23,0 & 23,0 & 23,0 & \\
\hline $\mathrm{pH}$ & 7,1 & 7,3 & 7,58 & 7,75 & 7,04 & 7,13 & 7,96 & 7,92 & 8 & 8,13 & \\
\hline
\end{tabular}




\begin{tabular}{|c|c|c|c|c|c|c|c|c|c|c|}
\hline & & Sistema 2 & C3 & Ciliados & & & & & & \\
\hline Amostra & dia 0. & dia 1 & dia 2 & dia 3 & dia 4 & dia 5 & dia 6 & dia 7. & dia 8 & dia 9 \\
\hline 1 & 0 & 0 & 0 & 0 & \begin{tabular}{|l|}
1151 \\
\end{tabular} & 71 & 10 & 0 & o & 0 \\
\hline 2 & 0 & 0 & 0 & 0 & 1112 & 77 & 8 & 0 & 0 & 0 \\
\hline 3 & 0 & 0 & 0 & 0 & 1214 & 75 & 4 & 0 & 0 & 0 \\
\hline 4 & 0 & 0 & 0 & 0 & 1110 & 85 & 8 & 0 & 0 & 0 \\
\hline 5 & 0 & 0 & 0 & 0 & 1223 & 66 & 12 & 0 & 0 & 0 \\
\hline 6 & 0 & 0 & 0 & 0 & 1252 & 60 & 10 & 0 & 0 & 0 \\
\hline 7 & 0 & 0 & 0 & 0 & 1182 & 55 & 0 & 0 & 0 & 0 \\
\hline 8 & 0 & 0 & 0 & 0 & 1100 & 76 & 0 & 0 & 0 & 0 \\
\hline 9 & 0 & 0 & 0 & 0 & 1224 & 63 & 2 & 0 & 0 & 0 \\
\hline 10 & 0 & 0 & 0 & 0 & 1010 & 66 & 9 & 0 & 0 & 0 \\
\hline 11 & 0 & 0 & 0 & 0 & 1070 & 66 & 10 & 0 & 0 & 0 \\
\hline 12 & 0 & 0 & 0 & 0 & 1037 & 54 & 12 & 0 & 0 & 0 \\
\hline Média & 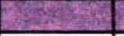 & 0,00 & 0,00 & 0,00 & 1140,42 & 67,83 & 6,81 & 0,00 & 0,00 & 0,00 \\
\hline Temp ${ }^{\circ} \mathrm{C}$ & 25 & 22,0 & 22,0 & 23,0 & 23,5 & 22,5 & 24,0 & 23,0 & 23,0 & 23,0 \\
\hline $\mathrm{pH}$ & 7,01 & 7,15 & \begin{tabular}{l|l}
7,22 \\
\end{tabular} & 7,39 & 7,7 & 7,78 & 7,79 & 8 & 8 & 8,17 \\
\hline
\end{tabular}

\section{Sistema $3-C_{3} \quad$ Ciliados}

\begin{tabular}{|c|c|c|c|c|c|c|c|c|c|c|}
\hline & & & & & & & & & & \\
\hline Amostra & dia 0 & dia 1 : & dia 2 & dia 3 & dia 4 & dia 5 & dia 6 & dia 7 & dia 8 & dia 9 \\
\hline 1 & 0 & $\begin{array}{l}0 \\
\end{array}$ & 0 & 0 & \begin{tabular}{|r|}
1256 \\
\end{tabular} & 44 & 10 & 0 & 0 & 0 \\
\hline 2 & 0 & 0 & 0 & 0 & 1250 & 61 & 7 & 20 & 0 & 0 \\
\hline 3 & 0 & 0 & 0 & 0 & 1244 & 40 & 9 & 20 & 0 & 0 \\
\hline 4 & 0 & 0 & 0 & 0 & 1236 & 31 & 8 & 15 & 0 & 0 \\
\hline 5 & 0 & 0 & 0 & 0 & 1240 & 36 & 9 & 15 & 0 & 0 \\
\hline 6 & 0 & 0 & 0 & 0 & 1239 & 41 & 8 & 10 & 0 & 0 \\
\hline 7 & 0 & 0 & 0 & 0 & 1132 & 46 & 7 & 10 & 0 & 0 \\
\hline 8 & of & 0 & 0 & 0 & 1274 & 52 & 0 & 10 & 0 & 0 \\
\hline 9 & 0 & 0 & 0 & 0 & 1190 & 35 & 0 & 10 & 0 & 0 \\
\hline 10 & 0 & 0 & 0 & 0 & 1254 & 37 & 10 & 12 & 0 & 0 \\
\hline 11 & 0 & 0 & 0 & 0 & 1286 & 27 & 16 & 10 & 0 & 0 \\
\hline 12 & 0 & 0 & 0 & 0 & 1290 & 41 & 14 & 12 & 0 & 0 \\
\hline Média & & 0,00 & 0,00 & 0,00 & 1240,92 & $40 ; 92$ & 8.17 & 12,00 & 0.00 & 0,00 \\
\hline Temp ${ }^{\circ} \mathrm{C}$ & 25,0 & 22,0 & 22,0 & 23,0 & 23,5 & 22,5 & 24,0 & 23,0 & 23,0 & 23,0 \\
\hline $\mathrm{pH}$ & 6,95 & 7,03 & 7,41 & 7,45 & 7,66 & 7,79 & 7,79 & 7,9 & 7,94 & 8,1 \\
\hline
\end{tabular}

\begin{tabular}{|c|c|c|c|c|c|c|c|c|c|c|}
\hline \multirow{2}{*}{\multicolumn{2}{|c|}{$\sqrt{1-a^{2}+m}$}} & \multicolumn{2}{|c|}{ Sistema $4 . \mathrm{C3}$} & \multicolumn{7}{|l|}{ Ciliados } \\
\hline & dia 0 & dia 1 & dia 2 & dia 3 & dia 4 & dia 5. & dia 6 & dia 7 & dia 8 & dia 9 \\
\hline 1 & 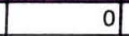 & 0 & 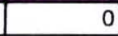 & 100 & $\begin{array}{r}910 \\
\end{array}$ & 87 & 5 & 0 & 0 & 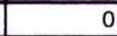 \\
\hline 2 & 0 & 0 & 0 & 100 & 935 & 91 & 6 & 0 & 0 & 0 \\
\hline 3 & 0 & 0 & 0 & 100 & 900 & 83 & 10 & 0 & 0 & 0 \\
\hline 4 & 0 & 0 & 0 & 200 & 910 & 77 & 12 & 0 & 0 & 0 \\
\hline 5 & 0 & 0 & 0 & 200 & 915 & 87 & 9 & 0 & 0 & 0 \\
\hline 6 & 0 & 0 & 0 & 200 & 945 & 61 & 5 & 0 & 0 & 0 \\
\hline 7 & 0 & 0 & 0 & 100 & 965 & 69 & 6 & 0 & 0 & 0 \\
\hline 8 & 0 & 0 & 0 & 200 & 1056 & 57 & 7 & 0 & 0 & 0 \\
\hline 9 & 0 & 0 & 0 & 100 & 1015 & 84 & 9 & 0 & 0 & 0 \\
\hline 10 & 0 & 0 & 0 & 200 & 930 & 89 & 9 & 0 & 0 & 0 \\
\hline 11 & 0 & 0 & 0 & 200 & 956 & 92 & 11 & 0 & 0 & 0 \\
\hline 12 & 0 & 0 & 0 & 200 & 905 & 95 & 0 & 0 & 0 & 0 \\
\hline Média & & 0,00 & 0,00 & 158,33 & 945,17 & 81,00 & 7,42 & 0,00 & 0,00 & 0,00 \\
\hline Temp ${ }^{\circ} \mathrm{C}$ & 25 & 22,0 & 22,0 & 23,0 & 23,5 & 22,5 & 24,0 & 23,0 & 22,0 & 23,0 \\
\hline $\mathrm{pH}$ & 6,87 & 7,12 & 7,36 & 7,42 & 7,68 & 7,84 & 7,7 & 7,94 & 7,96 & 8.09 \\
\hline
\end{tabular}

\begin{tabular}{|c|c|c|c|c|c|c|c|c|c|c|}
\hline & & Sistema $5-C$ & & Ciliados & & & & & & \\
\hline Amostra & dia 0 & dia 1 & dia 2 & dia 3 & dia 4 & dia 5 & dia 6. & dia 7 & dia 8 & dia 9 \\
\hline 1 & 0 & 0 & 0 & 196 & 872 & 21 & 0 & 0 & 0 & 0 \\
\hline 2 & 0 & 0 & 0 & 314 & 887 & 23 & 0 & 0 & 0 & 0 \\
\hline 3 & 0 & 0 & 0 & 305 & 962 & 14 & 0 & 0 & 0 & 0 \\
\hline 4 & 0 & 0 & 0 & 225 & 952 & 16 & 2 & 0 & 0 & 0 \\
\hline 5 & 0 & 0 & 0 & 274 & 994 & 17 & 5 & 0 & 0 & 0 \\
\hline 6 & 0 & 0 & 0 & 275 & 912 & 19 & 6 & 0 & 0 & 0 \\
\hline 7 & 0 & 0 & 0 & 286 & 914 & 22 & 10 & 0 & 0 & 0 \\
\hline 8 & 0 & 0 & 0 & 312 & 890 & 26 & 0 & 0 & 0 & 0 \\
\hline 9 & 0 & 0 & 0 & 278 & 909 & 32 & 0 & 0 & 0 & 0 \\
\hline 10 & 0 & 0 & 0 & 212 & 925 & 11 & 6 & 0 & 0 & 0 \\
\hline 11 & 0 & 0 & 0 & 225 & 862 & 12 & 0 & 0 & 0 & 0 \\
\hline 12 & 0 & 0 & 0 & 205 & 957 & 10 & 5 & 0 & 0 & 0 \\
\hline Média & & 0,00 & 0,00 & 258,92 & 919,67 & 18,58 & 2,83 & 0,00 & 0,00 & 0,00 \\
\hline Temp ${ }^{\circ} \mathrm{C}$ & 25 & 22,0 & 22,0 & 23,0 & 23,5 & 225 & 24,0 & 23,0 & 22,0 & 23,0 \\
\hline $\mathrm{pH}$ & 6,9 & 7,19 & 7,35 & 7,53 & 7,64 & 7,69 & 7.78 & 8,12 & 8,12 & 8 \\
\hline
\end{tabular}


Experimento III

\begin{tabular}{|c|c|c|c|c|c|c|c|c|c|c|c|}
\hline & & Sistema & $1 \mathrm{~B}$ & Bactérias & Filamentc & tosas & & & & & \\
\hline Amostra & dia 0. & dia 1 & dia 2 & dia 3 & dia 4 & dia 5 & dia 6 & dia 7 & dia 8 & dia 9 & \\
\hline 1 & 0 & $\begin{array}{r}1 \\
\end{array}$ & 6 & 9 & 3 & 0 & 2 & 2 & 1 & 0 & 1,50 \\
\hline 2 & 0 & 2 & 3 & 10 & 3 & 0 & 2 & 3 & 2 & 0 & 2,92 \\
\hline 3 & 0 & 2 & 2 & 9 & 3 & 0 & 1 & 1 & 1 & 2 & 9,08 \\
\hline 4 & 0 & 1 & 4 & 9 & 6 & 0 & 2 & 1 & 1 & 0 & 7,67 \\
\hline 5 & 0 & 1 & 4 & 11 & 8 & 1 & 2 & 1 & 2 & 0 & 0,75 \\
\hline 6 & 0 & 2 & 3 & 5 & 11 & 2 & 3 & 2 & 1 & 2 & 2,00 \\
\hline 7 & 0 & 1 & 2 & 9 & 15 & 0 & 1 & 1 & 1 & 0 & 1,17 \\
\hline 8 & 0 & 1 & 2 & 9 & 14 & 0 & 2 & 1 & 1 & 2 & 1,58 \\
\hline 9 & 0 & 1 & 2 & 9 & 10 & 0 & 3 & 1 & 2 & 2 & 1,00 \\
\hline 10 & 0 & 2 & 3 & 9 & 3 & 0 & 2 & 0 & 3 & 2 & \\
\hline 11 & 0 & 2 & 3 & 9 & 10 & 2 & 2 & 1 & 3 & 1 & \\
\hline 12 & 0 & 2 & 1 & 11 & 6 & 4 & 2 & 0 & 1 & 1 & \\
\hline Média & 0 & 1,50 & 2,92 & 9,08 & 7,67 & 0,75 & 2,00 & 1,17 & 1,58 & 1,00 & \\
\hline Temp ${ }^{\circ} \mathrm{C}$ & 24 & 23,0 & 22,0 & 22,0 & 23,0 & 25,0 & 24,0 & 24,0 & 22,0 & 20,0 & OD(final) \\
\hline $\mathrm{pH}$ & 6,96 & 7 & 7 & 7,13 & 7,74 & 7,98 & 7,97| & 8 & 8 & 8,14 & $\mathrm{mg} / \mathrm{L}$ \\
\hline
\end{tabular}

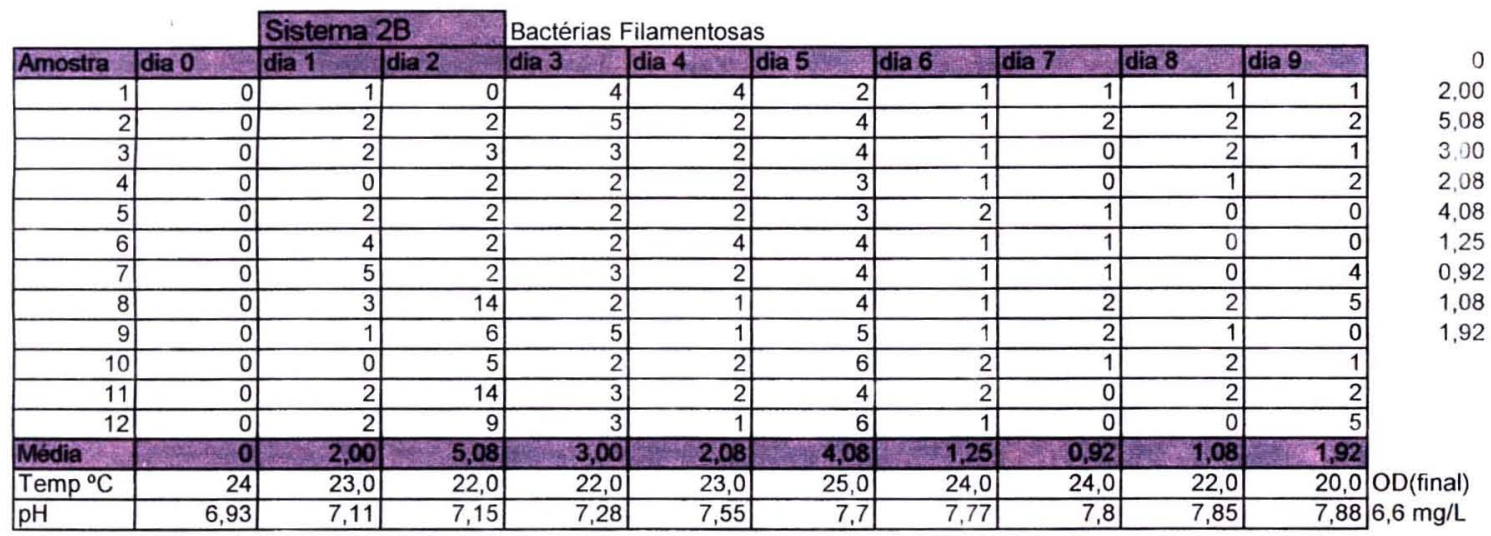

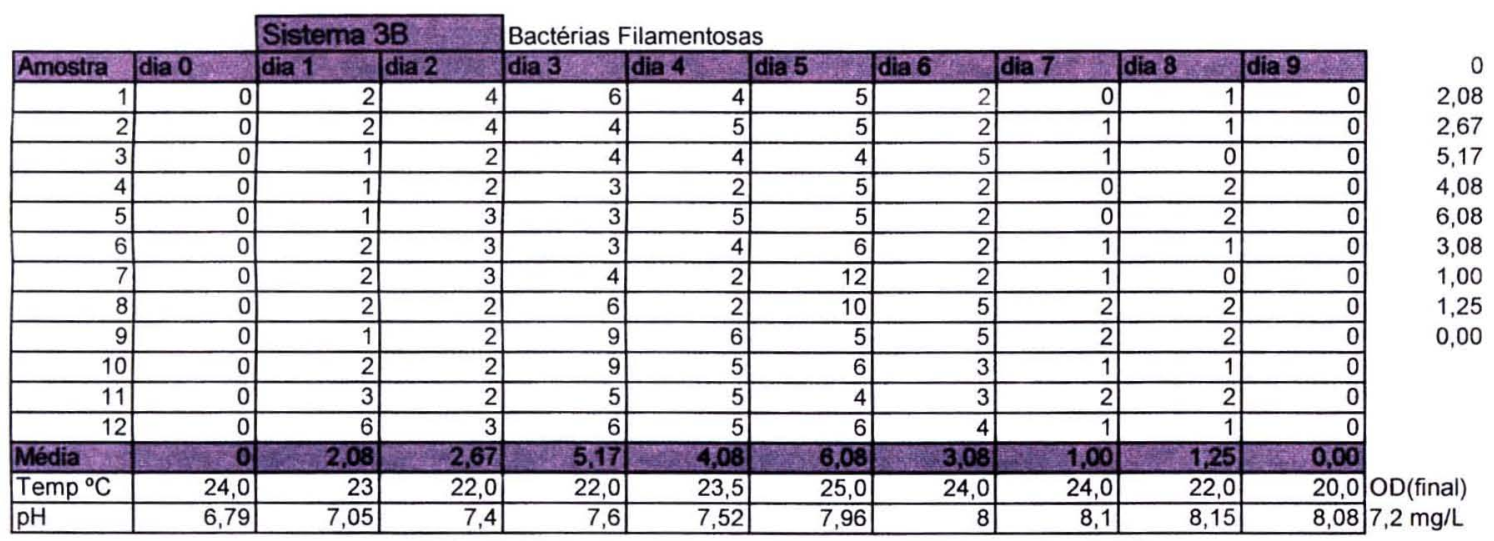

\begin{tabular}{|c|c|c|c|c|c|c|c|c|c|c|c|}
\hline & & Sistema & & Bactérias F & Filamentosa & & & & & & \\
\hline Amostra & dia 0 & dia 1 & dia 2 & dia 3 & dia 4 & dia 5 & dia 6 & dia 7 & dia 8 & dia9 & 0 \\
\hline 1 & 0 & 1 & 2 & 12 & 4 & 6 & 0 & 1 & 1 & 1 & 2,00 \\
\hline 2 & 0 & 2 & 4 & 15 & 4 & 4 & 0 & 2 & 1 & 1 & 3,83 \\
\hline 3 & 0 & 2 & 3 & 16 & 4 & 6 & 1 & 2 & 2 & 1 & 10,08 \\
\hline 4 & 0 & 2 & 2 & 15 & 4 & 4 & 0 & 2 & 2 & 1 & 4,00 \\
\hline 5 & 0 & 2 & 3 & 10 & 6 & 5 & 0 & 1 & 2 & 1 & 6,08 \\
\hline 6 & 0 & 2 & 2 & 9 & 3 & 4 & 0 & 2 & 3 & 1 & 0,67 \\
\hline 7 & 0 & 3 & 5 & 6 & 2 & 9 & 2 & 2 & 3 & 2 & 1,83 \\
\hline 8 & 0 & 3 & 2 & 11 & 3 & 11 & 1 & 2 & 2 & 1 & 2,00 \\
\hline 9 & 0 & 2 & 2 & 9 & 4 & 7 & 1 & 1 & 2 & 1 & 1,42 \\
\hline 10 & 0 & 1 & 3 & 3 & 4 & 7 & 1 & 2 & 2 & 1 & \\
\hline 11 & 0 & 2 & 6 & 4 & 4 & 4 & 1 & 2 & 2 & 2 & \\
\hline 12 & 0 & 2 & 12 & 11 & 6 & 6 & 1 & 3 & 2 & 4 & \\
\hline Média & & 2,00 & 3,83 & 10,08 & 4.00 & 6,08 & 0,67 & 1,83 & 2,00 & 1,42 & \\
\hline Temp ${ }^{\circ} \mathrm{C}$ & 24,0 & 23 & 22,0 & 22,0 & 23,0 & 25,0 & 24,0 & 24,0 & 22,0 & 20,0 & OD(final) \\
\hline $\mathrm{pH}$ & 6,86 & 7,15 & 7,22 & 7,2 & 7,65 & 7,79 & 7,9 & 7,9 & 7,94 & 7,96 & $\mathrm{mg} / \mathrm{L}$ \\
\hline
\end{tabular}


Sistema 5B Bactérias Filamentosas

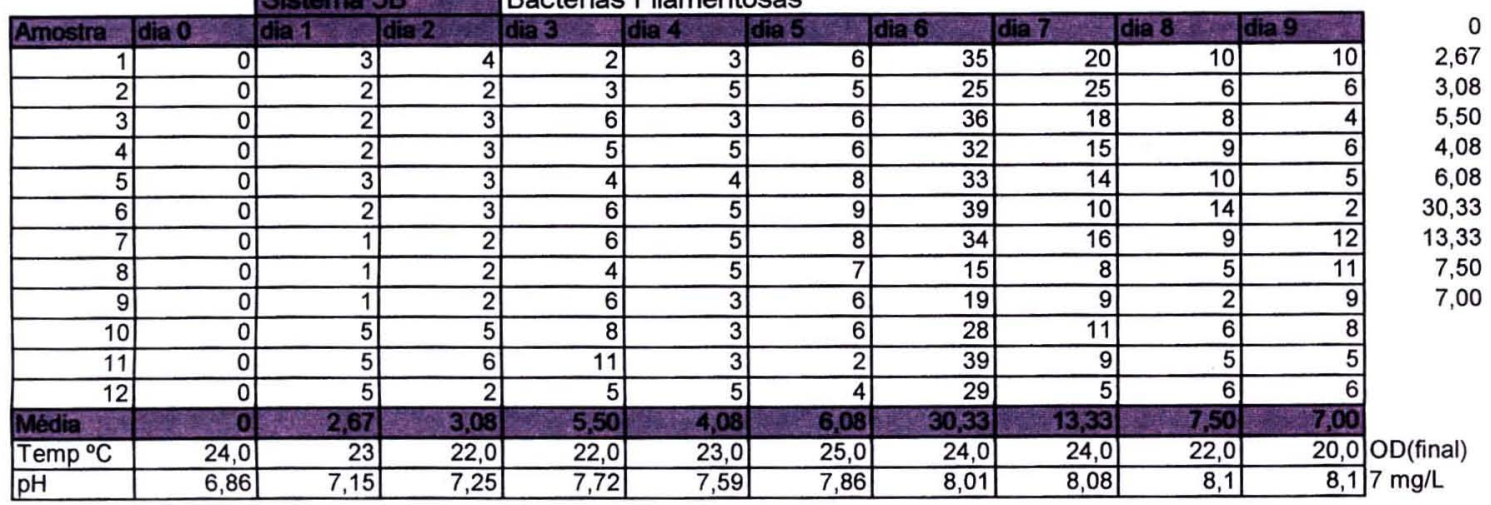

\section{Quantidade de microrganismos/substrato DBO de $350 \mathrm{mg} / \mathrm{l}$ aeraçäo continua}

\section{$0,62 \mathrm{~m} /$ - Inseticida DDVP}

\begin{tabular}{|c|c|c|c|c|c|c|c|c|c|c|c|}
\hline & & Sistema & $11-1$ & Bacterias F & Filamentosas & & & & & & \\
\hline Amostra & dia 0 & diat & dia 2 & dia 3 & dia 4 & dia 5 & dia 6 & dia 7 & dia 8 & dia 9. & 0 \\
\hline 1 & 0 & 1 & 6 & 4 & 2 & 30 & 7 & 2 & 2 & 4 & 0,00 \\
\hline 2 & 0 & 1 & 4 & 4 & 4 & 33 & 6 & 4 & 3 & 2 & 4,92 \\
\hline 3 & 0 & 2 & 5 & 4 & 6 & 16 & 3 & 5 & 4 & 2 & 5,17 \\
\hline 4 & 0 & 2 & 6 & 6 & 4 & 31 & 5 & 6 & 5 & 4 & 4,33 \\
\hline 5 & 0 & 2 & 2 & 5 & 4 & 30 & 6 & 2 & 6 & 5 & 22,17 \\
\hline 6 & 0 & 1 & 6 & 4 & 4 & 15 & 4 & 5 & 5 & 2 & 6,08 \\
\hline 7 & 0 & 1 & 6 & 4 & 4 & 15 & 9 & 4 & 4 & 5 & 4,00 \\
\hline 8 & 0 & 1 & 6 & 3 & 4 & 12 & 2 & 6 & 2 & 6 & 4,00 \\
\hline 9 & 0 & 1 & 6 & 9 & 4 & 18 & 5 & 2 & 5 & 4 & 4,25 \\
\hline 10 & 0 & 2 & 4 & 7 & 5 & 32 & 8 & 3 & 4 & 6 & \\
\hline 11 & 0 & 1 & 4 & 6 & 4 & 22 & 9 & 5 & 4 & 6 & \\
\hline 12 & 0 & 1 & 4 & 6 & 7 & 12 & 9 & 4 & 4 & 5 & \\
\hline Média & 0 & 0,00 & 4,92 & 5,17 & 4,33 & 22,17 & 6,08 & 4,00 & 4,00 & 4,25 & \\
\hline $\operatorname{Temp}^{\circ} \mathrm{C}$ & 24 & 23,0 & 22,0 & 22,0 & 23,0 & 25,0 & 24,0 & 24,0 & 22,0 & 20,0 & $O D$ (final) \\
\hline $\mathrm{pH}$ & 6,88 & 7,3 & 7,2 & 7,3 & 7,59 & 7,92 & 7,9 & 7,92 & 7,95 & 7,98 & $6,6 \mathrm{mg} / \mathrm{L}$ \\
\hline
\end{tabular}

\begin{tabular}{|c|c|c|c|c|c|c|c|c|c|c|c|}
\hline & & Sistemal & $2 \mid-1$ & Bactérias F & illamentosa & & & & & & \\
\hline Amostra & dia 0 & dia 1 & dia 2 & dia 3 & dia 4 & dia 5 & dia 6 & dia 7 & dia 8 & dia 9 & 0 \\
\hline 1 & 0 & 2 & 1 & 8 & 6 & 10 & 7 & 5 & 4 & 2 & 3,33 \\
\hline 2 & 0 & 4 & 2 & 6 & 5 & 11 & 7 & 6 & 5 & 4 & 3,25 \\
\hline 3 & 0 & 2 & 3 & 5 & 4 & 10 & 10 & 4 & 6 & 6 & 6,25 \\
\hline 4 & 0 & 2 & 2 & 6 & 2 & 10 & 7 & 5 & 4 & 5 & 6,00 \\
\hline 5 & 0 & 2 & 2 & 9 & 5 & 10 & 5 & 6 & 7 & 4 & 10,25 \\
\hline 6 & 0 & 2 & 2 & 4 & 4 & 11 & 9 & 8 & 8 & 4 & 6,00 \\
\hline 7 & 0 & 5 & 2 & 9 & 6 & 12 & 9 & 4 & 5 & 5 & 5,83 \\
\hline 8 & 0 & 2 & 2 & 9 & 6 & 16 & 4 & 4 & 6 & 2 & 6,00 \\
\hline 9 & 0 & 5 & 5 & 9 & 9 & 9 & 4 & 8 & 7 & 2 & 3,50 \\
\hline 10 & 0 & 6 & 6 & 5 & 10 & 9 & 2 & 8 & 7 & 2 & \\
\hline 11 & 0 & 6 & 6 & 2 & 7 & 11 & 2 & 6 & 7 & 3 & \\
\hline 12 & 0 & 2 & 6 & 3 & 8 & 4 & 6 & 6 & 6 & 3 & \\
\hline Média & 0 & 3,33 & 3,25 & 6,25 & 6,00 & 10,25 & 6,00 & 5,83 & 6,00 & 3,50 & \\
\hline Temp ${ }^{\circ} \mathrm{C}$ & 24 & 23,0 & 22,0 & 22,0 & 23,0 & 25,0 & 24,0 & 24,0 & 22,0 & 20,0 & OD(final) \\
\hline & 6,88 & 7,1 & 7,12 & 7,22 & 7,13 & 7,69 & 7,92 & 8 & 8,08 & 8,1 & $6 \mathrm{mg} / \mathrm{L}$ \\
\hline
\end{tabular}

\begin{tabular}{|c|c|c|c|c|c|c|c|c|c|c|c|}
\hline & & Sistema 3 & $3-1$ & Bactérias F & ilamentosa & & & & & & \\
\hline Amostra & dia 0 & dia 1 & dia 2 & dia 3 & dia 4 & dia 5 & dia 6 & dia 7 & dia 8 & dia 9 & \\
\hline \begin{tabular}{|r|}
1 \\
\end{tabular} & 0 & 1 & 1 & 2 & 0 & 6 & 2 & 4 & 4 & 6 & \\
\hline 2 & 0 & 2 & 2 & 3 & 0 & 4 & 2 & 5 & 2 & 7 & 2,08 \\
\hline 3 & 0 & 3 & 2 & 2 & 0 & 6 & 2 & 6 & 3 & 8 & 3,75 \\
\hline 4 & 0 & 3 & 3 & 1 & 0 & 4 & 2 & 4 & 3 & 4 & 0,50 \\
\hline 5 & 0 & 3 & 3 & 1 & 1 & 6 & 4 & 5 & 3 & 8 & 6,17 \\
\hline 6 & 0 & 1 & 3 & 6 & 1 & 6 & 6 & 3 & 4 & 6 & 3,08 \\
\hline 7 & 0 & 1 & 2 & 2 & 1 & 8 & 6 & 3 & 6 & 7 & 3,5 \\
\hline 8 & 0 & 1 & 2 & 5 & 1 & 8 & 5 & 3 & 5 & 10 & 4,4 \\
\hline 9 & 0 & 3 & 2 & 5 & 1 & 4 & 4 & 2 & 6 & 6 & \\
\hline 10 & 0 & 3 & 2 & 6 & 0 & 6 & 2 & 1 & 5 & 5 & \\
\hline 11 & 0 & 6 & 2 & 6 & 0 & 6 & 1 & 3 & 6 & 4 & \\
\hline 12 & 0 & 4 & 1 & 6 & 1 & 10 & 1 & 3 & 6 & 4 & \\
\hline Média & 0 & 2,58 & 2,08 & 3,75 & 0.50 & 6,17 & 3,08 & 3,50 & 4,42 & 6,25 & \\
\hline Temp $^{\circ} \mathrm{C}$ & 24 & 23,0 & 22,0 & 22,0 & 23,0 & 25,0 & 24,0 & 24,0 & 22,0 & 20,0 & $O D$ (final) \\
\hline $\mathrm{pH}$ & 6,88 & 6,95 & 6,9 & 6,51 & 7,7 & 6,9 & 7,57 & 7,69 & 7,8 & 7,9 & $6,8 \mathrm{mg} / \mathrm{L}$ \\
\hline
\end{tabular}




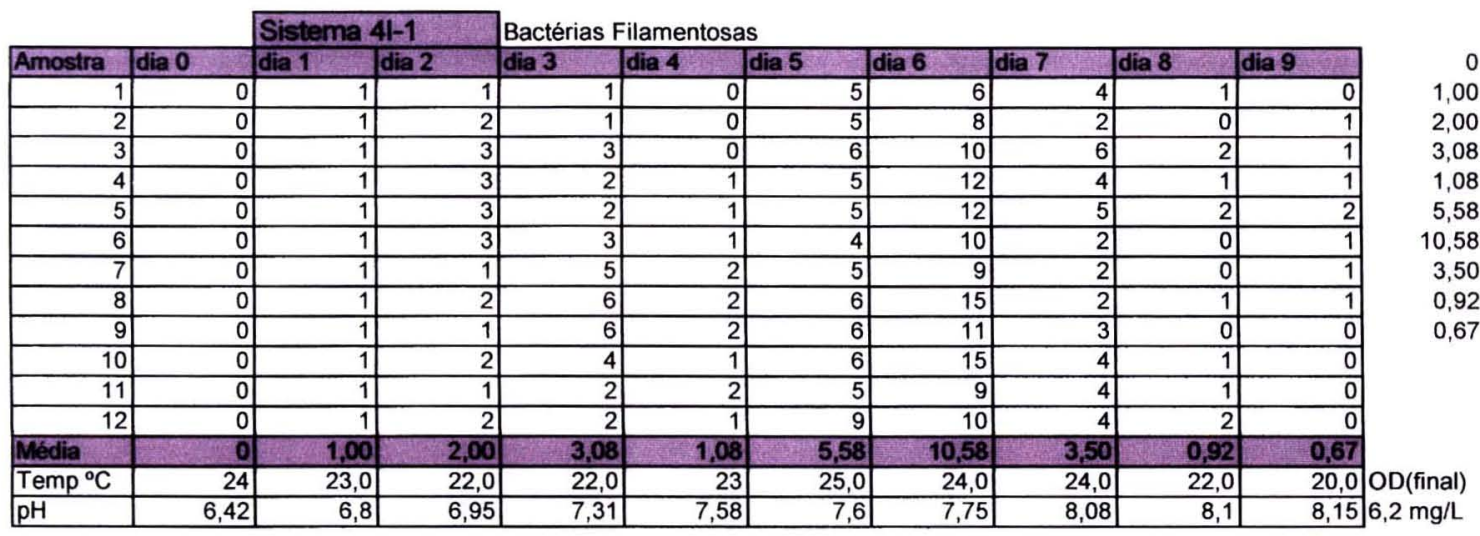

\begin{tabular}{|c|c|c|c|c|c|c|c|c|c|c|c|}
\hline & & Sistema 5 - & & Bactérias F & ilamentosa & & & & & & \\
\hline Amostra & dia 0 & $\operatorname{dia} 1$ & dia 2 & dia 3 & dia 4 & dia 5 & dia 6 & dia 7 & dia 8 & dia 9 & 0 \\
\hline \begin{tabular}{r|}
1 \\
\end{tabular} & 0 & 0 & 1 & 9 & 1 & 8 & 5 & 1 & 2 & 1 & 2,25 \\
\hline 2 & 0 & 1 & 1 & 7 & 1 & 6 & 2 & 1 & 2 & 2 & 2,00 \\
\hline 3 & 0 & 0 & 1 & 5 & 2 & 2 & 1 & 2 & 2 & 4 & 5,33 \\
\hline 4 & 0 & 1 & 5 & 4 & 1 & 6 & 1 & 2 & 2 & 2 & 2,00 \\
\hline 5 & 0 & 2 & 4 & 6 & 1 & 4 & 5 & 4 & 2 & 2 & 5,92 \\
\hline 6 & 0 & 6 & 5 & 5 & 2 & 5 & 5 & 4 & 2 & 2 & 5,33 \\
\hline 7 & 0 & 2 & 1 & 5 & 6 & 2 & 9 & 4 & 2 & 4 & 2,33 \\
\hline 8 & 0 & 3 & 1 & 5 & 4 & 9 & 9 & 4 & 2 & 2 & 2,00 \\
\hline 9 & 0 & 2 & 1 & 6 & 1 & 8 & 9 & 2 & 2 & 2 & 2,08 \\
\hline 10 & 0 & 2 & 2 & 4 & 2 & 9 & 9 & 1 & 2 & 1 & \\
\hline 11 & 0 & 4 & 1 & 4 & 2 & 6 & 4 & 1 & 2 & 1 & \\
\hline 12 & 0 & 4 & 1 & 4 & 1 & 6 & 5 & 2 & 2 & 2 & \\
\hline \begin{tabular}{|l} 
Média \\
\end{tabular} & 0 & 2,25 & 2,00 & 5,33 & 2,00 & 5,92 & 5,33 & 2,33 & 2,00 & 2,08 & \\
\hline Temp $^{\circ} \mathrm{C}$ & 24 & 23,0 & 22,0 & 22,0 & 23,0 & 25,0 & 24,0 & 24,0 & 22 & 20,0 & OD(final) \\
\hline $\mathrm{pH}$ & 6,8 & 7,5 & 7,67 & 7,97 & 7,73 & 7,75 & 7,91 & 8 & 8,12 & 8,1 & $6,66 \mathrm{mg} / \mathrm{l}$ \\
\hline
\end{tabular}

\section{Quantidade de microrganlsmos/substrato DBO de $350 \mathrm{mg} / \mathrm{V}$ aeraçäo continua}

$1,25 \mathrm{~m} /$ - Insettcida DDVP

\begin{tabular}{|c|c|c|c|c|c|c|c|c|c|c|c|}
\hline & & Sistema 1f- & & Bactérias F & Filamentosa & & & & & & \\
\hline Amostra & dila 0 & dia 1 & dia 2 & dia 3 & dia 4 & dia 5 & dia 6 & dia 7 & dia 8 & dia 9 & \\
\hline 1 & 0 & 2 & 2 & & 20 & 6 & 5 & 2 & 2 & 0 & 2,00 \\
\hline 2 & 0 & 2 & 2 & 4 & 12 & 13 & 5 & 2 & 1 & 0 & 2,08 \\
\hline 3 & 0 & 2 & 2 & 1 & 15 & 15 & 6 & 2 & 可 & 1 & 3,27 \\
\hline 4 & 0 & 2 & 2 & 1 & 16 & 20 & 6 & 4 & 2 & 2 & 15,17 \\
\hline 5 & 0 & 3 & 2 & 7 & 14 & 11 & 5 & 3 & 1 & 2 & 14,25 \\
\hline 6 & 0 & 2 & 3 & 6 & 11 & 14 & 5 & 2 & 1 & 2 & 5,50 \\
\hline 7 & 0 & 2 & 2 & 3 & 11 & 13 & 6 & 1 & 0 & 2 & 2,33 \\
\hline 8 & 0 & 2 & 4 & 4 & 10 & 15 & 6 & 1 & 0 & 4 & 0,00 \\
\hline 9 & 0 & 2 & 2 & 5 & 16 & 14 & 6 & 2 & 0 & 1 & 1,58 \\
\hline 10 & 0 & 2 & 2 & 2 & 15 & 10 & 4 & 3 & 2 & 0 & \\
\hline 11 & 0 & 3 & 1 & 2 & 17 & 15 & 6 & 3 & 2 & 2 & \\
\hline 12 & 0 & 0 & 1 & 1 & 25 & 25 & 6 & 3 & 0 & 3 & \\
\hline Média & 0 & 2,00 & 2,08 & 3,27 & 15,17 & 14,25 & 5,50 & 2,33 & & 1,58 & \\
\hline Temp ${ }^{\circ} \mathrm{C}$ & 24 & 23,0 & 22,0 & 22,0 & 23,0 & 25 & 24,0 & 24,0 & 22,0 & 20 & (final) \\
\hline $\mathrm{pH}$ & 6,84 & 7,15 & 7,33 & 7,4 & 7,65 & 7,93 & 7,93 & 7,94 & 7,95 & 7,96 & $6 \mathrm{mg} / \mathrm{L}$ \\
\hline
\end{tabular}

\begin{tabular}{|c|c|c|c|c|c|c|c|c|c|c|c|}
\hline & & Sistema 21 & & Bactérias F & Filamentosas & & & & & & \\
\hline Amostra & $\widehat{\text { dia } 0}$ & dia 1 & $\overline{d i a} 2$ & dia 3 & dia 4 & dia 5 & dia 6 & dia 7 & dia 8 & dia 9 & 0 \\
\hline \begin{tabular}{r|}
1 \\
\end{tabular} & 0 & 1 & 1 & 2 & 4 & 5 & 10 & 16 & 2 & 1 & 1,83 \\
\hline 2 & 0 & 1 & 1 & & 2 & 4 & 20 & 15 & 2 & 2 & 1,33 \\
\hline 4 & 0 & 2 & 1 & 2 & 3 & 8 & 30 & 12 & 2 & 1 & 3,08 \\
\hline 5 & 0 & 2 & 1 & 2 & 3 & 6 & 25 & 10 & 2 & 2 & 8,00 \\
\hline 6 & 0 & 1 & 1 & 2 & 3 & 8 & 24 & 9 & 2 & 1 & 23,75 \\
\hline 9 & 0 & 1 & 2 & 3 & 2 & 9 & 26 & 10 & 2 & 2 & 1,75 \\
\hline 10 & 0 & 2 & 1 & 2 & 2 & 10 & 24 & 6 & 3 & 2 & \\
\hline 11 & 0 & 3 & 2 & 2 & 2 & 11 & 23 & 5 & 3 & 2 & \\
\hline 12 & 0 & 3 & 2 & 2 & 2 & 13 & 33 & 5 & 3 & 4 & \\
\hline Média & 0 & 1,83 & 1,33 & 2,09 & 3,08 & 8,00 & 23,75 & 10,17 & 2,25 & 1,75 & \\
\hline Temp ${ }^{\circ} \mathrm{C}$ & 24 & 23,0 & 22,0 & 22,0 & 23,0 & 25 & 24,0 & 24,0 & 22,0 & 20,0 & $\mathrm{OD}$ (final) \\
\hline
\end{tabular}



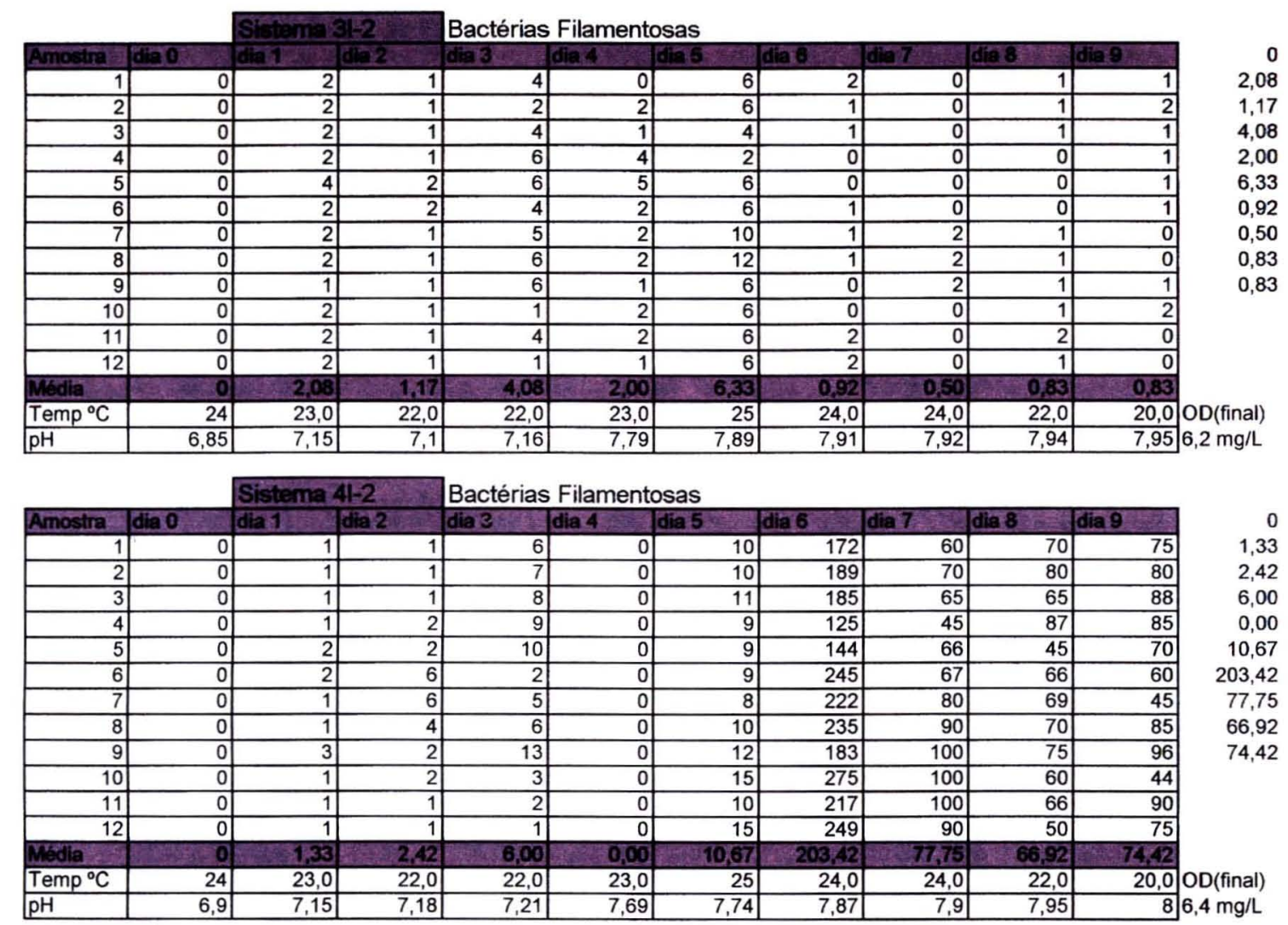

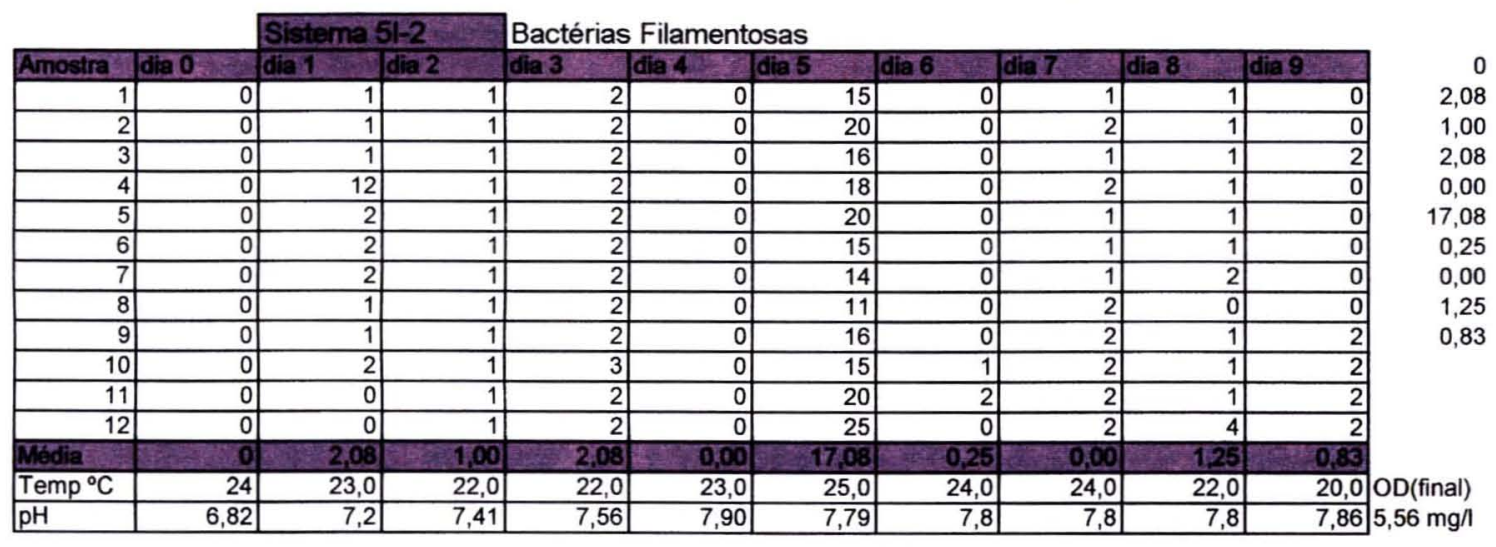

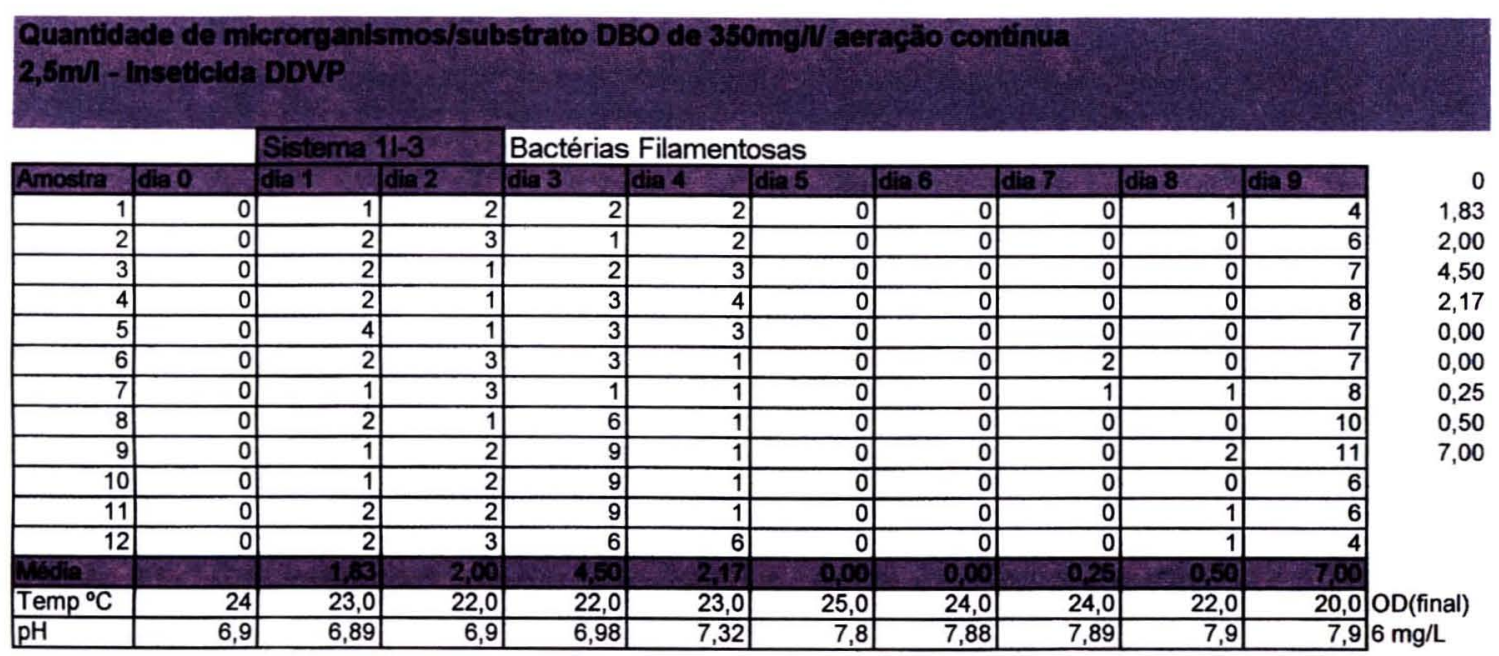




\begin{tabular}{|c|c|c|c|c|c|c|c|c|c|c|c|}
\hline & & Sistema: & $21-3$ & Bactérias & Filament & tosas & & & & & \\
\hline Amostra & dia 0 & dia 1 & dia 2 & dia 3 & dia 4 & dia 5 & dia 6 & dia 7 & dia 8 & dia 9 & 0 \\
\hline 1 & 0 & 2 & 2 & 3 & 4 & 4 & 0 & 0 & 1 & 6 & 1,58 \\
\hline 2 & 0 & 1 & 4 & 2 & 0 & 0 & 0 & 0 & 2 & 2 & 3,17 \\
\hline 3 & 0 & 1 & 2 & 2 & 0 & 0 & 0 & 0 & 1 & 4 & 3,67 \\
\hline 4 & 0 & 1 & 2 & 2 & 1 & 0 & 0 & 0 & 0 & 5 & 1,33 \\
\hline 5 & 0 & 1 & 3 & 2 & 3 & 0 & 0 & 0 & 1 & 2 & 0,08 \\
\hline 6 & 0 & 2 & 6 & 2 & 2 & 0 & 0 & 0 & 2 & 6 & 0,00 \\
\hline 7 & 0 & 1 & 5 & 6 & 1 & 0 & 0 & 0 & 2 & 5 & 0,00 \\
\hline 8 & 0 & 1 & 4 & 5 & 1 & 0 & 0 & 0 & 1 & 5 & 1,17 \\
\hline 9 & 0 & 1 & 4 & 6 & 1 & 0 & 0 & 0 & 1 & 7 & 5,25 \\
\hline 10 & 0 & 6 & 4 & 4 & 2 & 0 & 0 & 0 & 1 & 7 & \\
\hline 11 & 0 & 1 & 1 & 4 & 1 & 0 & 0 & 0 & 1 & 7 & \\
\hline 12 & 0 & 1 & 1 & 6 & 0 & 0 & 0 & 0 & 1 & 7 & \\
\hline Média & & 1,58 & 3,17 & 3.67 & 1,33 & 0,08 & 0,00 & 0,00 & 1,17 & 5,25 & \\
\hline $\operatorname{Temp}^{\circ} \mathrm{C}$ & 24 & 23,0 & 22,0 & 22,0 & 23,0 & 25,0 & 24,0 & 24,0 & 22,0 & 20,0 & $\mathrm{OD}$ (final) \\
\hline $\mathrm{pH}$ & 6,87 & 7,1 & 7,32 & 7,44 & 7,6 & 7,7 & 7,77 & 7,8 & 7,9 & 7,9 & $6,4 \mathrm{mg} / \mathrm{l}$ \\
\hline
\end{tabular}

\begin{tabular}{|c|c|c|c|c|c|c|c|c|c|c|c|}
\hline & & Sistema: & $31-3$ & Bactérias & Filamentc & osas & & & & & \\
\hline Amostra & $\operatorname{dia} 0$ & dia 1 & dia 2 & dia 3 & dia 4 & dia 5 & dia 6 & $\operatorname{dia} 7$ & dia 8 & dia 9 & 0 \\
\hline \begin{tabular}{|r|}
1 \\
\end{tabular} & 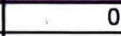 & 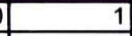 & 1 & 2 & 6 & 0 & 0 & 0 & 0 & 1 & 1,92 \\
\hline 2 & $\underline{0}$ & 2 & 1 & 2 & 6 & 0 & 0 & 0 & 0 & 1 & 2,33 \\
\hline 3 & $\underline{0}$ & 2 & 2 & 1 & 3 & 0 & 0 & 0 & 0 & 1 & 4,17 \\
\hline 4 & $\underline{0}$ & 2 & 2 & 3 & 3 & 0 & 0 & 0 & 0 & 2 & 4,33 \\
\hline 5 & $\underline{0}$ & 1 & 2 & 3 & 3 & 0 & 0 & 0 & 0 & 1 & 0,00 \\
\hline 6 & $\underline{0}$ & 1 & 4 & 5 & 3 & 0 & 0 & 0 & 0 & 0 & 0,00 \\
\hline 7 & 0 & 2 & 4 & 6 & 4 & 0 & 0 & 0 & 0 & 0 & 0,50 \\
\hline 8 & 0 & 4 & 0 & 6 & 5 & 0 & 0 & 2 & 0 & 1 & 0,00 \\
\hline 9 & 0 & 2 & 0 & 6 & 3 & 0 & 0 & 1 & 0 & 1 & 0,75 \\
\hline 10 & 0 & 2 & 0 & 6 & 5 & 0 & 0 & 1 & 0 & 1 & \\
\hline 11 & $\underline{0}$ & 2 & 6 & 4 & 6 & 0 & 0 & 1 & 0 & 0 & \\
\hline 12 & 0 & 2 & 6 & 6 & 5 & 0 & 0 & 1 & 0 & 0 & \\
\hline Média & & 1,92 & 2,33 & 4,17 & 4,33 & 0,00 & 0,00 & 0,50 & 0,00 & 0,75 & \\
\hline Temp ${ }^{\circ} \mathrm{C}$ & 24 & 23,0 & 22,0 & 22,0 & 23,0 & 25,0 & 24,0 & 24,0 & 22,0 & 20,0 & $\mathrm{OD}$ (final) \\
\hline pH & 6,85 & 7 & 7,1 & 7,17 & 6,91 & 6,63 & 6,63 & 6,7 & 6,7 & 6,72 & $5,2 \mathrm{mg} / \mathrm{l}$ \\
\hline
\end{tabular}

\begin{tabular}{|c|c|c|c|c|c|c|c|c|c|c|c|c|}
\hline & & & Sistema 4 & $41-3$ & Bactérias F & ilamentosas & & & & & & \\
\hline Amostra & dia 0 & & dia 1 & dia 2 & dia 3 & dia 4 & dia 5 & $\operatorname{dia} 6$ & dia 7 & dia 8 & dia9 & 0 \\
\hline $\begin{array}{r}1 \\
\end{array}$ & & 0 & 1 & 2 & 3 & 2 & 0 & 0 & 0 & 0 & 0 & 1,00 \\
\hline 2 & & 0 & 2 & 2 & 2 & 2 & 0 & 0 & 0 & 0 & 0 & 1,83 \\
\hline 3 & & 0 & 2 & 1 & 2 & 2 & 0 & 0 & 0 & 0 & 0 & 2,00 \\
\hline 4 & & 0 & 0 & 1 & 3 & 6 & 0 & 0 & 0 & 0 & 0 & 1,92 \\
\hline 5 & & 0 & 0 & 2 & 1 & 1 & 0 & 0 & 0 & 0 & 0 & 0,00 \\
\hline 6 & & 0 & 2 & 1 & 1 & 2 & 0 & 0 & 0 & 0 & 0 & 0,00 \\
\hline 7 & & 0 & 0 & 1 & 3 & 2 & 0 & 0 & 0 & 0 & 0 & 0,00 \\
\hline 8 & & 0 & 1 & 2 & 4 & 2 & 0 & 0 & 0 & 0 & 0 & 0,00 \\
\hline 9 & & 0 & 1 & 2 & 2 & 1 & 0 & 0 & 0 & 0 & 0 & 0,00 \\
\hline 10 & & 0 & 1 & 2 & 1 & 1 & 0 & 0 & 0 & 0 & 0 & \\
\hline 11 & & 0 & 0 & 1 & 1 & 2 & 0 & 0 & 0 & 0 & 0 & \\
\hline 12 & & 0 & 2 & 5 & 1 & 0 & 0 & 0 & 0 & 0 & 0 & \\
\hline Mbdia & & & 1,00 & 1,83 & 2,00 & 1,92 & 0,00 & 0,00 & 0,00 & 0,00 & 0,00 & \\
\hline Temp ${ }^{\circ} \mathrm{C}$ & & 24 & 23,0 & 22,0 & 22,0 & 23,0 & 25,0 & 24,0 & 24,0 & 22,0 & 20,0 & OD(final) \\
\hline $\mathrm{pH}$ & & 84 & 7,15 & 7,26 & 7,3 & 7,59 & 7,42 & 7,57 & 7,57| & 7,67 & 7,9 & $6,2 \mathrm{mg} / \mathrm{l}$ \\
\hline
\end{tabular}

\begin{tabular}{|c|c|c|c|c|c|c|c|c|c|c|c|c|}
\hline & & Sisterma 5 & $5 !-3$ & & Bactérias F & ilamentosas & & & & & & \\
\hline Amostra & dia 0 & dia 1 & dia 2 & & dia 3 & $\begin{array}{ll}\text { dia } 4 \\
\end{array}$ & dia 5 & $\operatorname{dia} 6$ & dia 7 & dia 8 & dia 9 & 0 \\
\hline $\begin{array}{r} \\
\end{array}$ & 0 & 1 & & 1 & 1 & 1 & 0 & 0 & 1 & 0 & 0 & 1,25 \\
\hline 2 & 0 & 1 & & 1 & 2 & 1 & 0 & 0 & 1 & 0 & 0 & 0,92 \\
\hline 3 & 0 & 1 & & 2 & 2 & 1 & 0 & 0 & 0 & 0 & 0 & 2,33 \\
\hline 4 & 0 & 1 & & 1 & 2 & 2 & 0 & 0 & 0 & 0 & 0 & 0,92 \\
\hline 5 & 0 & 3 & & 1 & 1 & 1 & 1 & 0 & 0 & 0 & 0 & 0,08 \\
\hline 6 & 0 & 1 & & 1 & 2 & 1 & 0 & 0 & 1 & 0 & 0 & 0,00 \\
\hline 7 & 0 & 1 & & 2 & 2 & 1 & 0 & 0 & 1 & 0 & 0 & 0,42 \\
\hline 8 & 0 & 1 & & 1 & 1 & 2 & 0 & of & 0 & 0 & 0 & 0,00 \\
\hline 9 & $\underline{0}$ & 2 & & 1 & 2 & 1 & 0 & of & 0 & 0 & 0 & 0,00 \\
\hline 10 & $\underline{0}$ & 1 & & 0 & 4 & 0 & 0 & 0 & 1 & 0 & 0 & \\
\hline 11 & 0 & 1 & & 0 & 6 & 0 & 0 & 0 & 0 & 0 & 0 & \\
\hline 12 & $\overline{0}$ & 1 & & 0 & 3 & 0 & 0 & 0 & 0 & 0 & 0 & \\
\hline Modia & & 1,25 & & $\longdiv { 9 2 }$ & 2,33 & 0,92 & 0,08 & 0,00 & 0,42 & 0,00 & 0,00 & \\
\hline Temp ${ }^{\circ} \mathrm{C}$ & 24 & 23,0 & & 22,0 & 22,0 & 23,0 & 25,0 & 24,0 & 24,0 & 24,0 & 22,0 & OD(final) \\
\hline pH & 6,86 & 6,96 & & 7 & 7,1 & 7,3 & 8,11 & 8,12 & 8,12 & 8,12 & 8,1 & $5,6 \mathrm{mg} / \mathrm{L}$ \\
\hline
\end{tabular}


Experimento III

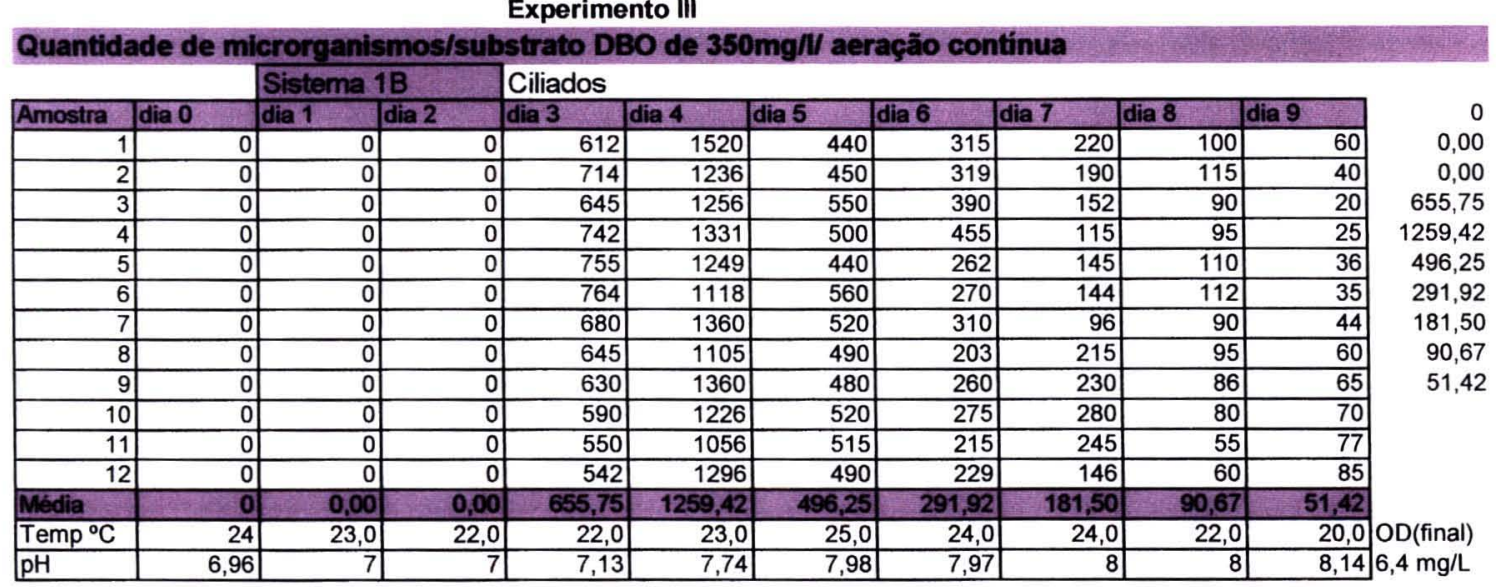

\begin{tabular}{|c|c|c|c|c|c|c|c|c|c|c|c|}
\hline & & Sistema: & & Ciliados & & & & & & & \\
\hline Amostra & dia 0 & dia 1 & dia 2 & dia 3 & $\operatorname{dia} 4$ & dia 5 & iia 6 & dia 7 & dia 8 & dia 9 & \\
\hline \begin{tabular}{|l}
1 \\
\end{tabular} & 0 & 0 & 0 & 655 & 765 & 345 & 290 & \begin{tabular}{|r|}
225 \\
\end{tabular} & 115 & 80 & 0,00 \\
\hline 2 & 0 & 0 & 0 & 642 & 910 & 360 & 212 & 215 & 145 & 110 & 0,00 \\
\hline 3 & 0 & 0 & 0 & 670 & 905 & 345 & 205 & 195 & 116 & 115 & 628,08 \\
\hline 4 & 0 & 0 & 0 & 668 & 755 & 300 & 213 & 225 & 118 & 125 & 857,92 \\
\hline 5 & 0 & 0 & 0 & 604 & 910 & 410 & 202 & 218 & 215 & 95 & 366,25 \\
\hline 6 & 0 & 0 & 0 & 470 & 955 & 405 & 258 & 180 & 202 & 99 & $248,5 \varepsilon$ \\
\hline 7 & 0 & 0 & 0 & 515 & 755 & 390 & 223 & 195 & 147 & 101 & 218,67 \\
\hline 8 & 0 & 0 & 0 & 601 & 900 & 395 & 256 & 208 & 148 & 115 & $153,0 \varepsilon$ \\
\hline 9 & 0 & 0 & 0 & 612 & 770 & 405 & 210 & 218 & 139 & 145 & 113,33 \\
\hline 10 & 0 & 0 & 0 & 650 & 777 & 360 & 330 & 215 & 165 & 165 & \\
\hline 11 & 0 & 0 & 0 & 752 & 995 & 330 & 315 & 315 & 169 & 115 & \\
\hline 12 & 0 & 0 & 0 & 698 & 898 & 350 & 269 & 215 & 158 & 95 & \\
\hline Média & 0 & 0,00 & 0,00 & 628,08 & 857,92 & 366,25 & 248,58 & 218,67 & 153,08 & 113,33 & \\
\hline $\operatorname{Temp}^{\circ} \mathrm{C}$ & 24 & 23,0 & 22,0 & 22,0 & 23,0 & 25,0 & 24,0 & 24,0 & 22,0 & 20,0 & $\mathrm{OD}$ (final) \\
\hline pH & 6,93 & 7,11 & 7,15 & 7,28 & 7,55 & 7,7 & 7,77 & 7,8 & 7,85 & 7,88 & $6,6 \mathrm{mg} / \mathrm{L}$ \\
\hline
\end{tabular}

\begin{tabular}{|c|c|c|c|c|c|c|c|c|c|c|c|}
\hline & & Sistema 3 & 3B & Ciliados & & & & & & & \\
\hline Amostra & dia 0 & dia 1 & dia 2 & dia 3 & dia 4 & dia 5 & dia 6 & dia 7 & dia 8 & dia 9 & 0 \\
\hline \begin{tabular}{r|}
1 \\
\end{tabular} & 0 & 0 & 0 & 225 & 953 & 490 & 220 & 110 & 44 & 40 & 0,00 \\
\hline 2 & 0 & 0 & 0 & 280 & 817 & 450 & 325 & 115 & 65 & 60 & 0,00 \\
\hline 3 & 0 & 0 & 0 & 225 & 930 & 445 & 350 & 115 & 66 & 45 & 259,67 \\
\hline 4 & 0 & 0 & 0 & 250 & 1222 & 465 & 214 & 215 & 67 & 42 & 1133,00 \\
\hline 5 & 0 & 0 & 0 & 190 & 1100 & 430 & 380 & 256 & 45 & 36 & 449,50 \\
\hline 6 & 0 & 0 & 0 & 275 & 1110 & 430 & 450 & 245 & 66 & 45 & 316,58 \\
\hline 7 & 0 & 0 & 0 & 338 & 1256 & 405 & 300 & 236 & 69 & 36 & 169,67 \\
\hline 8 & 0 & 0 & 0 & 340 & 1230 & 480 & 300 & 119 & 70 & 33 & 67,83 \\
\hline 9 & 0 & 0 & 0 & 278 & 1352 & 496 & 250 & 148 & 78 & 40 & 41,08 \\
\hline 10 & 0 & 0 & 0 & 255 & 1301 & 444 & 330 & 154 & 85 & 36 & \\
\hline 11 & 0 & 0 & 0 & 245 & 1210 & 420 & 310 & 157 & 96 & 45 & \\
\hline 12 & 0 & 0 & 0 & 215 & 1115 & 439 & 370 & 166 & 63 & 35 & \\
\hline Média & 0 & 0,00 & 0,00 & 259,67 & 1133,00 & 449,50 & 316,58 & 169,67 & 67,83 & 41,08 & \\
\hline Temp ${ }^{\circ} \mathrm{C}$ & 24,0 & 23 & 22,0 & 22,0 & 23,5 & 25,0 & 24,0 & 24,0 & 22,0 & 20,0 & $O D$ (final) \\
\hline pH & 6,79 & 7,05 & 7,4 & 7,6 & 7,52 & 7,96 & 8 & & & & $7,2 \mathrm{mg} / \mathrm{L}$ \\
\hline
\end{tabular}

\begin{tabular}{|c|c|c|c|c|c|c|c|c|c|c|c|}
\hline & & Sistema & & & & & & & & & \\
\hline Amostra & dia 0 & dia 1 & dia 2 & dia.3 & dia 4. & dia 5 & dia 6 & $\operatorname{dia} 7$ & dia 8 & dia 9 & 0 \\
\hline 1 & 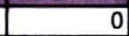 & 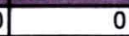 & 0 & 915 & 1088 & 750 & 360 & 215 & 44 & 20 & 0,00 \\
\hline 2 & 0 & 0 & 0 & 814 & 1222 & 760 & 315 & 222 & 46 & 25 & 0,00 \\
\hline 3 & 0 & 0 & 0 & 910 & 1180 & 800 & 325 & 236 & 54 & 35 & 833,58 \\
\hline 4 & 0 & 0 & 0 & 850 & 1316 & 710 & 330 & 196 & 51 & 30 & 1202,33 \\
\hline 5 & 0 & 0 & 0 & 860 & 1330 & 750 & 330 & 215 & 46 & 40 & 741,50 \\
\hline 6 & 0 & 0 & 0 & 855 & 1304 & 760 & 390 & 218 & 44 & 45 & 342,17 \\
\hline 7 & 0 & 0 & 0 & 870 & 1250 & 777 & 336 & 200 & 60 & 36 & 221,42 \\
\hline 8 & 0 & 0 & 0 & 960 & 1336 & 780 & 369 & 219 & 66 & 54 & 51,92 \\
\hline 9 & 0 & $\overline{0}$ & 0 & 770 & 1250 & 791 & 300 & 219 & 55 & 23 & 31,50 \\
\hline 10 & 0 & 0 & 0 & 740 & 1107 & 690 & 350 & 236 & 56 & 22 & \\
\hline 11 & 0 & 0 & 0 & 744 & 1095 & 680 & 356 & 245 & 31 & 26 & \\
\hline 12 & 0 & 0 & 0 & 715 & 950 & 650 & 345 & 236 & 70 & 22 & \\
\hline Média & 0 & 0,00 & 0,00 & 833,58 & 1202,33 & 741,50 & 342,17 & 221,42 & 51,92 & 31,50 & \\
\hline Temp ${ }^{\circ} \mathrm{C}$ & 24,0 & 23 & 22,0 & 22,0 & 23,0 & 25,0 & 24,0 & 24,0 & 22,0 & 20,0 & $\mathrm{OD}$ (final) \\
\hline $\mathrm{pH}$ & 6,86 & 7,15 & 7,22 & 7,2 & 7,65 & 7,79 & 7,9 & 7,9 & 7,94 & 7,96 & $7 \mathrm{mg} / \mathrm{L}$ \\
\hline
\end{tabular}




\begin{tabular}{|c|c|c|c|c|c|c|c|c|c|c|c|}
\hline & & Sistema 5 & & Ciliados & & & & & & & \\
\hline Amostra & dia 0 & dia 1 & dia 2 & dia 3 & dia 4 & dia 5 & dia 6 & dia 7 & dia 8 & dia 9 & 0 \\
\hline \begin{tabular}{r|}
1 \\
\end{tabular} & 0 & 0 & 0 & 215 & 910 & 790 & 450 & 315 & 95 & 40 & 0,00 \\
\hline 2 & 0 & 0 & 0 & 150 & 956 & 740 & 500 & 258 & 96 & 44 & 0,00 \\
\hline 3 & 0 & 0 & 0 & 155 & 963 & 690 & 430 & 269 & 85 & 42 & 177,00 \\
\hline 4 & 0 & 0 & 0 & 200 & 1270 & 660 & 440 & 245 & 88 & 45 & 935,00 \\
\hline 5 & 0 & 0 & 0 & 180 & 952 & 669 & 435 & 236 & 95 & 49 & 658,75 \\
\hline 6 & 0 & 0 & 0 & 190 & 912 & 554 & 415 & 258 & 105 & 55 & 454,58 \\
\hline 7 & 0 & 0 & 0 & 192 & 900 & 752 & 495 & 295 & 110 & 50 & 252,08 \\
\hline 8 & 0 & 0 & 0 & 188 & 856 & 760 & 446 & 247 & 102 & 61 & 95,33 \\
\hline 9 & 0 & 0 & 0 & 193 & 797 & 790 & 469 & 215 & 96 & 56 & 51,83 \\
\hline 10 & 0 & 0 & 0 & 156 & 804 & 490 & 460 & 226 & 92 & 63 & \\
\hline 11 & 0 & 0 & 0 & 160 & 910 & 445 & 450 & 225 & 89 & 61 & \\
\hline 12 & 0 & 0 & 0 & 145 & 990 & 565 & 465 & 236 & 91 & 56 & \\
\hline Média & 0 & 0,00 & 0,00 & 177,00 & 935,00 & 658,75 & 454,58 & 252,08 & 95,33 & 51,83 & \\
\hline Temp ${ }^{\circ} \mathrm{C}$ & 24,0 & 23 & 22,0 & 22,0 & 23,0 & 25,0 & 24,0 & 24,0 & 22,0 & 20,0 & $O D$ (final) \\
\hline $\mathrm{pH}$ & 6,86 & 7,15 & 7,25 & 7,72 & 7,59 & 7,86 & 8,01 & 8,08 & 8,1 & 8,1 & $7 \mathrm{mg} / \mathrm{L}$ \\
\hline
\end{tabular}

Quantidade de microrganismos/substrato DBO de $350 \mathrm{mg} / \mathrm{l}$ aeração continua
$0,62 \mathrm{~m} /$ - Inseticida DDVP

\begin{tabular}{|c|c|c|c|c|c|c|c|c|c|c|c|}
\hline & & Sistema 1 & $11-1$ & Ciliados & & & & & & & \\
\hline Amostra & dia 0 & dia 1 & dia 2 & dia 3 & dia 4 & dia 5 & dia 6 & dia 7 & dia 8 & dia 9 & 0 \\
\hline \begin{tabular}{|r|}
1 \\
\end{tabular} & 0 & 0 & 0 & 355 & \begin{tabular}{|r|}
1349 \\
\end{tabular} & 155 & 212 & 60 & 20 & 20 & 0,00 \\
\hline 2 & 0 & 0 & 0 & 260 & 1231 & 165 & 190 & 65 & 25 & 25 & 0,00 \\
\hline 3 & 0 & 0 & 0 & 268 & 1395 & 144 & 100 & 45 & 20 & 20 & 317,42 \\
\hline 4 & 0 & 0 & 0 & 331 & 1264 & 150 & 250 & 66 & 22 & 22 & 1350,67 \\
\hline 5 & 0 & 0 & 0 & 315 & 1191 & 165 & 115 & 44 & 33 & 19 & 149,00 \\
\hline 6 & 0 & 0 & 0 & 280 & 1360 & 145 & 225 & 49 & 31 & 15 & 199,25 \\
\hline 7 & 0 & 0 & 0 & 335 & 1450 & 155 & 195 & 56 & 32 & 19 & 60,25 \\
\hline 8 & 0 & 0 & 0 & 295 & 1156 & 165 & 175 & 61 & 20 & 23 & 23,67 \\
\hline 9 & 0 & 0 & 0 & 315 & 1456 & 132 & 212 & 66 & 21 & 22 & 22,17 \\
\hline 10 & 0 & 0 & 0 & 330 & 1440 & 122 & 250 & 70 & 19 & 24 & \\
\hline 11 & 0 & 0 & 0 & 380 & 1560 & 115 & 245 & 72 & 23 & 26 & \\
\hline 12 & 0 & 0 & 0 & 345 & 1356 & 175 & 222 & 69 & 18 & 31 & \\
\hline Média & 0 & 0,00 & 0,00 & 317,42 & 1350,67 & 149,00 & 199,25 & 60,25 & 23,67 & 22,17 & \\
\hline Temp ${ }^{\circ} \mathrm{C}$ & 24 & 23,0 & 22,0 & 22,0 & 23,0 & 25,0 & 24,0 & 24,0 & 22,0 & 20,0 & $\mathrm{OD}$ (final) \\
\hline $\mathrm{pH}$ & 6,88 & 7,3 & 7,2 & 7,3 & 7,59 & 7,92 & 7,9 & 7,92 & 7,95 & 7,98 & $6,6 \mathrm{mg} / \mathrm{L}$ \\
\hline
\end{tabular}

\begin{tabular}{|c|c|c|c|c|c|c|c|c|c|c|c|}
\hline & & Sistema 2 & $21-1$ & Ciliados & & & & & & & \\
\hline Amostra & Tdia 0 & dia 1 & dia 2 & dia 3 & dia 4 & dia 5 & dia 6 & $\operatorname{dia} 7$ & dia 8 & dia 9 & 0 \\
\hline 1 & 0 & 0 & 0 & 715 & $\begin{array}{r}755 \\
\end{array}$ & 345 & 250 & 115 & 60 & 20 & 0,00 \\
\hline 2 & 0 & 0 & 0 & 680 & 732 & 332 & 245 & 118 & 67 & 22 & 0,00 \\
\hline 3 & 0 & 0 & 0 & 660 & 660 & 350 & 225 & 132 & 77 & 40 & 662,08 \\
\hline 4 & 0 & 0 & 0 & 710 & 711 & 342 & 223 & 152 & 61 & 36 & 713,92 \\
\hline 5 & 0 & 0 & 0 & 690 & 701 & 290 & 254 & 142 & 75 & 35 & 361,92 \\
\hline 6 & 0 & 0 & 0 & 682 & 700 & 298 & 255 & 136 & 74 & 41 & 247,08 \\
\hline 7 & 0 & 0 & 0 & 645 & 642 & 256 & 260 & 123 & 69 & 39 & 139,00 \\
\hline 8 & 0 & 0 & 0 & 630 & 655 & 360 & 240 & 159 & 56 & 34 & 71,25 \\
\hline 9 & 0 & 0 & 0 & 625 & 756 & 415 & 235 & 146 & 85 & 32 & 32,92 \\
\hline 10 & 0 & 0 & 0 & 600 & 760 & 445 & 320 & 165 & 80 & 29 & \\
\hline 11 & 0 & 0 & 0 & 618 & 770 & 450 & 222 & 136 & 75 & 35 & \\
\hline 12 & 0 & 0 & 0 & 690 & 725 & 460 & 236 & 144 & 76 & 32 & \\
\hline Média & 0 & 0,00 & 0,00 & 662,08 & 713,92 & 361,92 & 247,08 & 139,00 & 71,25 & 32,92 & \\
\hline $\operatorname{Temp}^{\circ} \mathrm{C}$ & 24 & 23,0 & 22,0 & 22,0 & 23,0 & 25,0 & 24,0 & 24,0 & 22,0 & 20,0 & $O D$ (final) \\
\hline pH & 6,88 & 7,1 & 7,12 & 7,22 & 7,13 & 7,69 & 7,92 & 8 & 8,08 & 8,1 & $6 \mathrm{mg} / \mathrm{L}$ \\
\hline
\end{tabular}

\begin{tabular}{|c|c|c|c|c|c|c|c|c|c|c|c|}
\hline & & Sistema 3 & $31-1$ & Ciliados & & & & & & & \\
\hline Amostra & dia 0 & dia 1 & dia 2 & \begin{tabular}{|l|l|}
$d i a 3$ \\
\end{tabular} & dia 4 Tc & dia 5 & dia 6 & $\operatorname{dia} 7$ & dia 8. & dia 9 & 0 \\
\hline 1 & 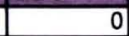 & 0 & 0 & 600 & 966 & 255 & 115 & 50 & 35 & 25 & 0,00 \\
\hline 2 & 0 & 0 & 0 & 550 & 890 & 245 & 142 & 65 & 36 & 45 & 0,00 \\
\hline 3 & 0 & 0 & 0 & 552 & 892 & 236 & 128 & 55 & 34 & 25 & 588,18 \\
\hline 4 & 0 & 0 & 0 & 615 & 902 & 236 & 156 & 56 & 44 & 30 & 809,67 \\
\hline 5 & 0 & 0 & 0 & 545 & 925 & 205 & 125 & 61 & 42 & 35 & 246,83 \\
\hline 6 & 0 & 0 & 0 & 570 & 725 & 200 & 123 & 69 & 36 & 44 & 130,42 \\
\hline 7 & 0 & 0 & 0 & 577 & 765 & 250 & 136 & 72 & 30 & 36 & 60,58 \\
\hline 8 & 0 & 0 & 0 & 578 & 774 & 260 & 125 & 70 & 32 & 30 & 39,25 \\
\hline 9 & 0 & 0 & 0 & 622 & 710 & 254 & 153 & 66 & 44 & 25 & 35,50 \\
\hline 10 & 0 & 0 & 0 & 623 & 712 & 245 & 119 & 56 & 42 & 46 & \\
\hline 11 & 0 & 0 & 0 & 638 & 755 & 289 & 121 & 54 & 45 & 44 & \\
\hline 12 & 0 & 0 & 0 & & 700 & 287 & 122 & 53 & 51 & 41 & \\
\hline Média & 0 & 0,00 & 0.00 & 588,18 & 809,67 & 246,83 & 130,42 & 60,58 & 39,25 & 35,50 & \\
\hline Temp ${ }^{\circ} \mathrm{C}$ & 24 & 23,0 & 22,0 & 22,0 & 23,0 & 25,0 & 24,0 & 24,0 & 22,0 & 20,0 & $D($ final) \\
\hline $\mathrm{pH}$ & 6,88 & 6,95 & 6,9 & 6,51 & 7,7 & 6,9 & 7,57 & 7,69 & 7,8 & 7,9 & $3,8 \mathrm{mg} / \mathrm{L}$ \\
\hline
\end{tabular}


Ciliados

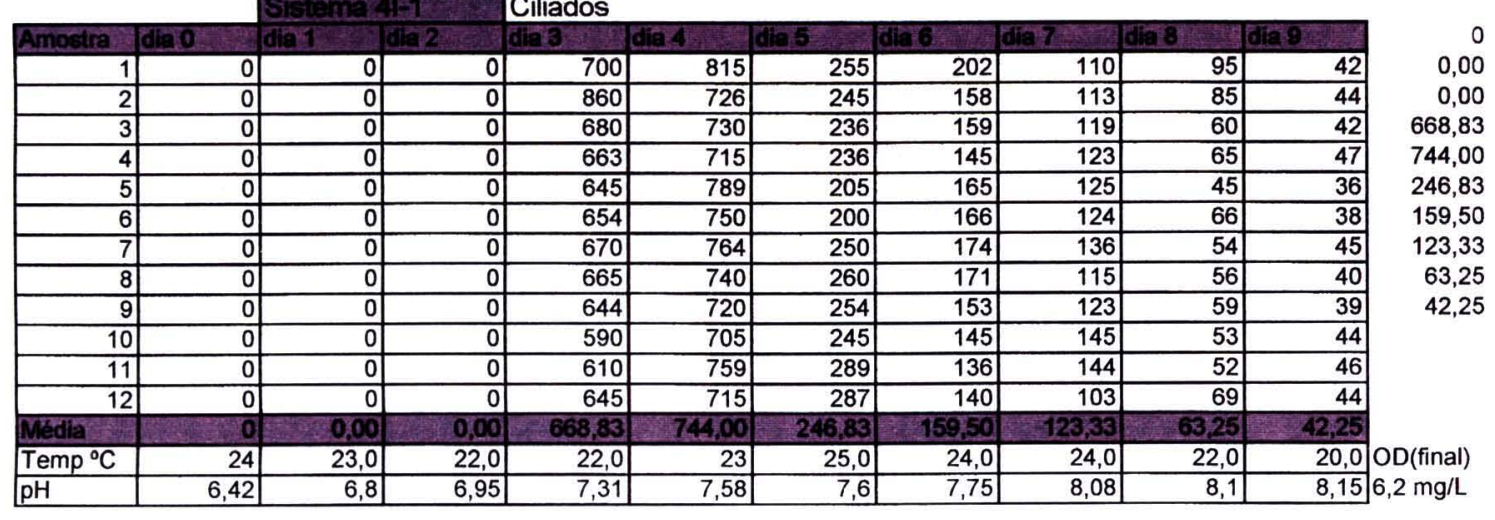

\begin{tabular}{|c|c|c|c|c|c|c|c|c|c|c|c|}
\hline & & Sistema 5 & $5 \mid-1$ & Ciliados & & & & & & & \\
\hline Amostra & dia 0 & 0 & Jdia 2 & dia 3 & jia 4 & dia 5 & dia 6 & dia 7 & dia 8 & dia 9 & \\
\hline 1 & 1 & 0 & 0 & 350 & 1150 & 356 & \begin{tabular}{|r|}
155 \\
\end{tabular} & 146 & \begin{tabular}{|r|}
145 \\
\end{tabular} & 212 & 0,00 \\
\hline 2 & 0 & 0 & 0 & 345 & 1122 & 330 & 160 & 165 & 165 & 190 & 0,00 \\
\hline 3 & 0 & 0 & 0 & 360 & 1094 & 366 & 110 & 154 & 144 & 200 & 329,58 \\
\hline 4 & 0 & 0 & 0 & 290 & 1053 & 350 & 115 & 169 & 145 & 185 & 1164,42 \\
\hline 5 & 0 & 0 & 0 & 330 & 1087 & 345 & 160 & 170 & 165 & 170 & 310,75 \\
\hline 6 & 0 & 0 & 0 & 345 & 1125 & 336 & 140 & 175 & 160 & 180 & 144,25 \\
\hline 7 & 0 & 0 & 0 & 360 & 1299 & 325 & 144 & 165 & 170 & 190 & 164,25 \\
\hline 8 & 0 & 0 & 0 & 366 & 1210 & 226 & 150 & 158 & 165 & 200 & 160,42 \\
\hline 9 & 0 & 0 & 0 & 278 & 1153 & 280 & 155 & 125 & 174 & 210 & 192,58 \\
\hline 10 & 0 & 0 & 0 & 285 & 1125 & 245 & 160 & 136 & 160 & 205 & \\
\hline 11 & 0 & 0 & 0 & 281 & 1365 & 260 & 140 & 196 & 155 & 195 & \\
\hline 12 & 0 & 0 & 0 & 365 & 1190 & 310 & 142 & 212 & 177 & 174 & \\
\hline Média & 0 & 0,00 & 0,00 & 329,58 & 1164,42 & 310,75 & 144.25 & 164,25 & 160,42 & 192,58 & \\
\hline Temp ${ }^{\circ} \mathrm{C}$ & 24 & 23,0 & 22,0 & 22,0 & 23,0 & 25,0 & 24,0 & 24,0 & 22,0 & 23,0 & DD(final) \\
\hline $\mathrm{pH}$ & 6,8 & 7,5 & 7,67 & 7,97 & 7,73 & 7,75 & 7,91 & 8 & 8,12 & 8,16 & $6,66 \mathrm{mg} / \mathrm{l}$ \\
\hline
\end{tabular}

\begin{tabular}{|c|c|c|c|c|c|c|c|c|c|c|c|}
\hline & & Sistema 1 & $11-2$ & Ciliados & & & & & & & \\
\hline Amostra & dia 0 & dia 1 & dia 2 & $\begin{array}{ll}\text { dia } 3 \\
\end{array}$ & 24 & dia 5 & dia 6. & dia 7 & lia 8 & dia9. & \\
\hline 1 & 0 & 0 & 0 & \begin{tabular}{|r|}
255 \\
\end{tabular} & 875 & 154 & 101 & 115 & 122 & 115 & 0,00 \\
\hline 2 & $\underline{0}$ & 0 & 0 & 170 & 946 & 133 & 110 & 126 & 123 & 125 & 0,00 \\
\hline 3 & $\overline{0}$ & 0 & 0 & 180 & 912 & 165 & 115 & 136 & 135 & 136 & 227,42 \\
\hline 4 & 0 & 0 & 0 & 190 & 887 & 164 & 99 & 118 & 136 & 145 & 900,58 \\
\hline 5 & 0 & 0 & 0 & 245 & 833 & 155 & 115 & 128 & 145 & 125 & 143,42 \\
\hline 6 & 0 & 0 & of & 325 & 966 & 160 & 99 & 136 & 115 & 136 & 102,17 \\
\hline 7 & 0 & 0 & 0 & 225 & 912 & 125 & 95 & 110 & 123 & 145 & 124,75 \\
\hline 8 & 0 & 0 & 0 & 315 & 981 & 115 & 89 & 125 & 125 & 175 & 131,17 \\
\hline 9 & 0 & 0 & 0 & 195 & 845 & 105 & 96 & 119 & 136 & 123 & 145,17 \\
\hline 10 & 0 & 0 & 0 & 202 & 833 & 145 & 92 & 123 & 163 & 166 & \\
\hline 11 & & 0 & 0 & 212 & 907 & 144 & 110 & 125 & 115 & 156 & \\
\hline 12 & 0 & 0 & 0 & 215 & 910 & 156 & 105 & 136 & 136 & 195 & \\
\hline Média & & 0,00 & 0,00 & 227,42 & 900,58 & 143,42 & 102,17 & 124,75 & & & \\
\hline Temp ${ }^{\circ} \mathrm{C}$ & 24 & 23,0 & 22,0 & 22,0 & 23,0 & 25 & 24,0 & 24,0 & 22,0 & 20 & $\mathrm{D}$ (final) \\
\hline $\mathrm{pH}$ & 6,84 & 7,15 & 7,33 & 7,4 & 7,65 & 7,93 & 7,93 & 7,94 & 7,95 & 7,96 & $\mathrm{mg} / \mathrm{L}$ \\
\hline
\end{tabular}

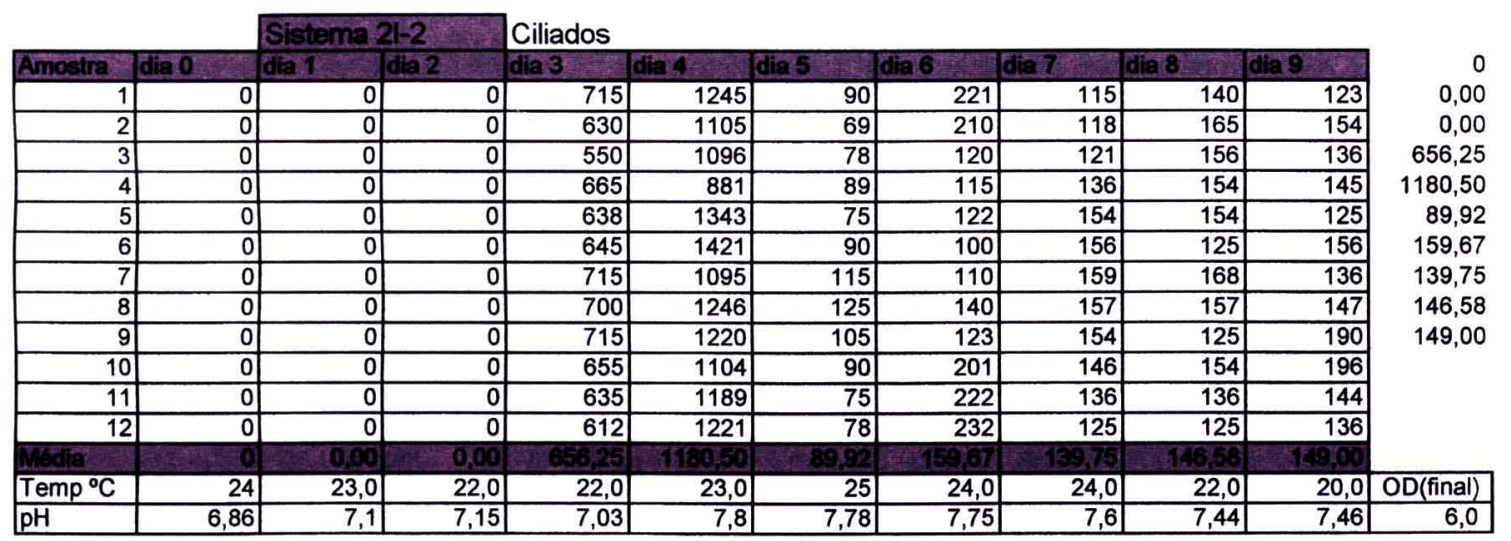


Ciliados

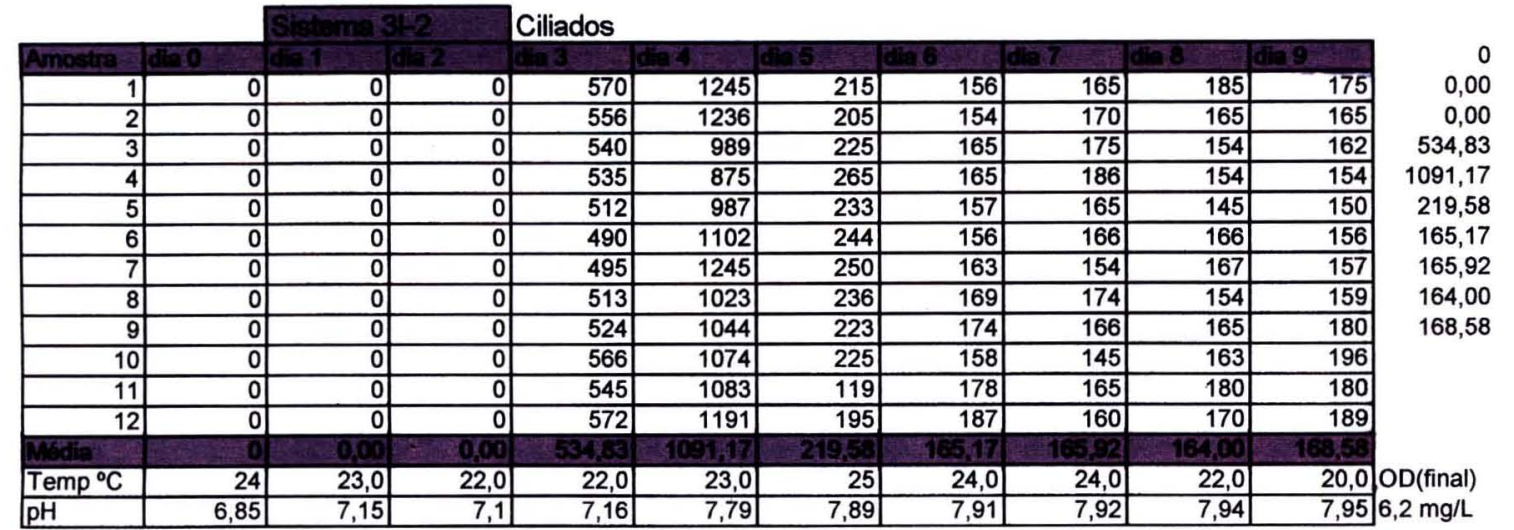

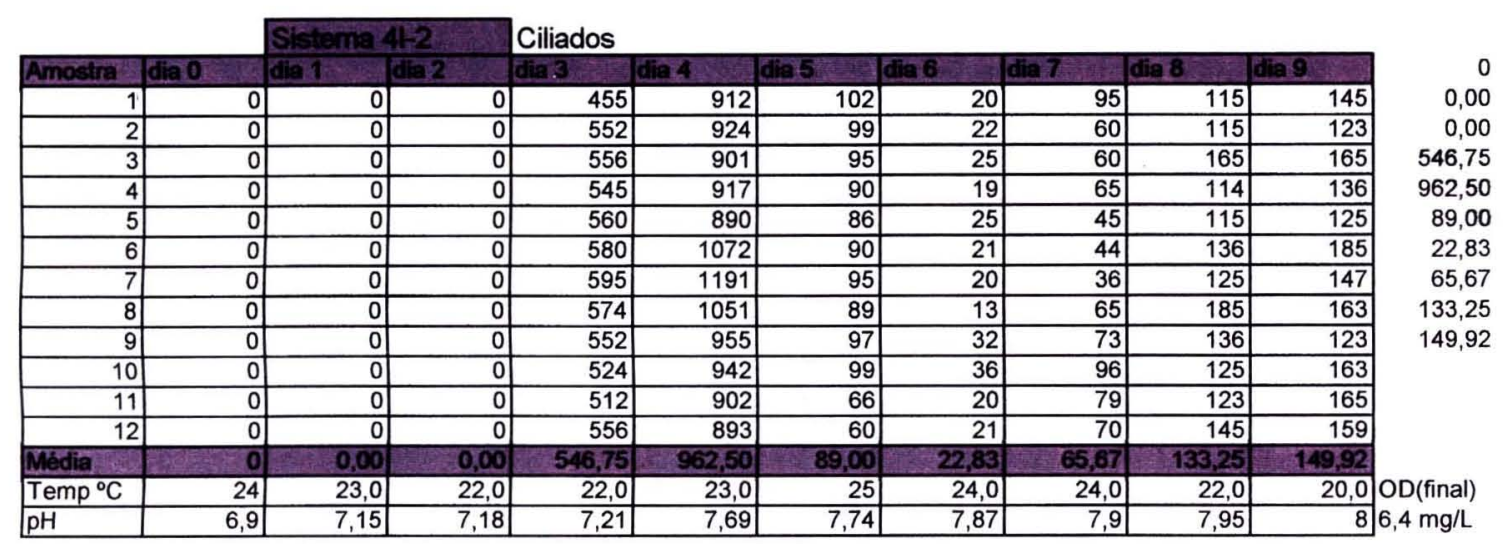

\begin{tabular}{|c|c|c|c|c|c|c|c|c|c|c|c|}
\hline & & Sistema & $51-2$ & Ciliados & & & & & & & \\
\hline Amostra & dia 0 & dia 1 & dia 2 & dia 3 & dia4 & dia 5 T & dia 6 & dia 7 & dia 8 & dia 9 & 0 \\
\hline 1 & 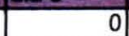 & 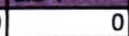 & 0 & 884 & 1185 & 220 & 55 & 89 & 115 & 142 & 0,00 \\
\hline 2 & 0 & 0 & 0 & 870 & 1055 & 223 & 53 & 80 & 152 & 136 & 0,00 \\
\hline 3 & 0 & 0 & 0 & 866 & 1162 & 250 & 56 & 89 & 113 & 145 & 826,08 \\
\hline 4 & 0 & 0 & 0 & 854 & 1254 & 260 & 65 & 99 & 112 & 123 & 1151,17 \\
\hline 5 & 0 & 0 & 0 & 790 & 1244 & 301 & 45 & 90 & 102 & 123 & 260,50 \\
\hline 6 & 0 & 0 & 0 & 775 & 1042 & 321 & 65 & 90 & 130 & 132 & 51,42 \\
\hline 7 & 0 & 0 & 0 & 877 & 1002 & 368 & 44 & 90 & 140 & 115 & 90,92 \\
\hline 8 & 0 & 0 & 0 & 812 & 1191 & 256 & 50 & 92 & 113 & 116 & 115,58 \\
\hline 9 & 0 & 0 & 0 & 854 & 1314 & 254 & 52 & 90 & 103 & 121 & 123,92 \\
\hline 10 & 0 & 0 & 0 & 836 & 1152 & 258 & 54 & 90 & 102 & 103 & \\
\hline 11 & 0 & 0 & 0 & 763 & 1103 & 215 & 42 & 96 & 113 & 105 & \\
\hline 12 & 0 & 0 & 0 & 732 & 1110 & 200 & 36 & 96 & 92 & 126 & \\
\hline Media & 0 & 0,00 & 0,00 & 826,08 & 1151,17 & 26050 & 51,42 & 90,92 & 115,58 & 123,92 & \\
\hline $\mathrm{Temp}^{\circ} \mathrm{C}$ & 24 & 23,0 & 22,0 & 22,0 & 23,0 & 25,0 & 24,0 & 24,0 & 22,0 & 20,0 & $\mathrm{DD}$ (final) \\
\hline $\mathrm{pH}$ & 6,82 & 7,2 & 7,41 & 7,56 & 7,90 & 7,79 & 7,8 & 7,8 & 7,8 & 7,86 & $5,56 \mathrm{mg} / \mathrm{l}$ \\
\hline
\end{tabular}

\begin{tabular}{|c|c|c|c|c|c|c|c|c|c|c|c|}
\hline \multicolumn{12}{|c|}{$\begin{array}{l}\text { Quantidade de microrgan } \\
2,5 \mathrm{~m} / \text { - Inseticida DDVP }\end{array}$} \\
\hline & \multirow[b]{2}{*}{ dia o } & \multirow{2}{*}{\multicolumn{2}{|c|}{\begin{tabular}{|l|l|} 
Sistema $11-3$ \\
dia 1 & dia 2
\end{tabular}}} & \multirow{2}{*}{\begin{tabular}{|l|} 
Ciliados \\
dia 3 \\
\end{tabular}} & \multirow[b]{2}{*}{ dia 4 } & \multirow[b]{2}{*}{ dia 5} & \multirow[b]{2}{*}{ dia 6} & \multirow[b]{2}{*}{ dia? } & \multirow{2}{*}{\multicolumn{2}{|c|}{\begin{tabular}{|l|l|} 
dia 8 dia 9 \\
\end{tabular}}} & \multirow{2}{*}{0} \\
\hline Amostra & & & & & & & & & & & \\
\hline 1 & 0 & \begin{tabular}{|r|}
0 \\
\end{tabular} & 0 & 515 & 1122 & 0 & 0 & 20 & 44 & 185 & 0,00 \\
\hline 2 & 0 & 0 & 0 & 495 & 1155 & 0 & 0 & 23 & 40 & 170 & 0,00 \\
\hline 3 & 0 & 0 & 0 & 521 & 892 & 0 & 0 & 25 & 65 & 180 & 510,33 \\
\hline 4 & 0 & 0 & 0 & 523 & 954 & 0 & 0 & 30 & 90 & 174 & 1019,83 \\
\hline 5 & 0 & 0 & 0 & 523 & 922 & 0 & 0 & 32 & 80 & 165 & 0,00 \\
\hline 6 & 0 & 0 & 0 & 536 & 942 & 0 & 0 & 30 & 95 & 195 & 0,00 \\
\hline 7 & 0 & 0 & 0 & 547 & 954 & 0 & 0 & 14 & 86 & 170 & 25,33 \\
\hline 8 & 0 & 0 & 0 & 499 & 1051 & 0 & 0 & 22 & 75 & 170 & 76,75 \\
\hline 9 & 0 & 0 & 0 & 501 & 1074 & 0 & 0 & 36 & 96 & 160 & 175,25 \\
\hline 10 & 0 & 0 & 0 & 512 & 1235 & 0 & 0 & 30 & 88 & 154 & \\
\hline 11 & 0 & 0 & 0 & 487 & 963 & 0 & 0 & 22 & 80 & 160 & \\
\hline 12 & 0 & 0 & 0 & 465 & 974 & 0 & 0 & 20 & 82 & 220 & \\
\hline & & & & & & 0,00 & & 25,33 & & & \\
\hline Temp ${ }^{\circ} \mathrm{C}$ & 24 & 23,0 & 22,0 & 22,0 & 23,0 & 25,0 & 24,0 & 24,0 & 22,0 & 20,0 & $\mathrm{OD}$ (final) \\
\hline $\mathrm{pH}$ & 6,82 & 6,89 & 6,9 & 6,98 & 7,32 & 7,8 & 7,88 & 7,89 & 7,9 & 7,9 & $3 \mathrm{mg} / \mathrm{L}$ \\
\hline
\end{tabular}




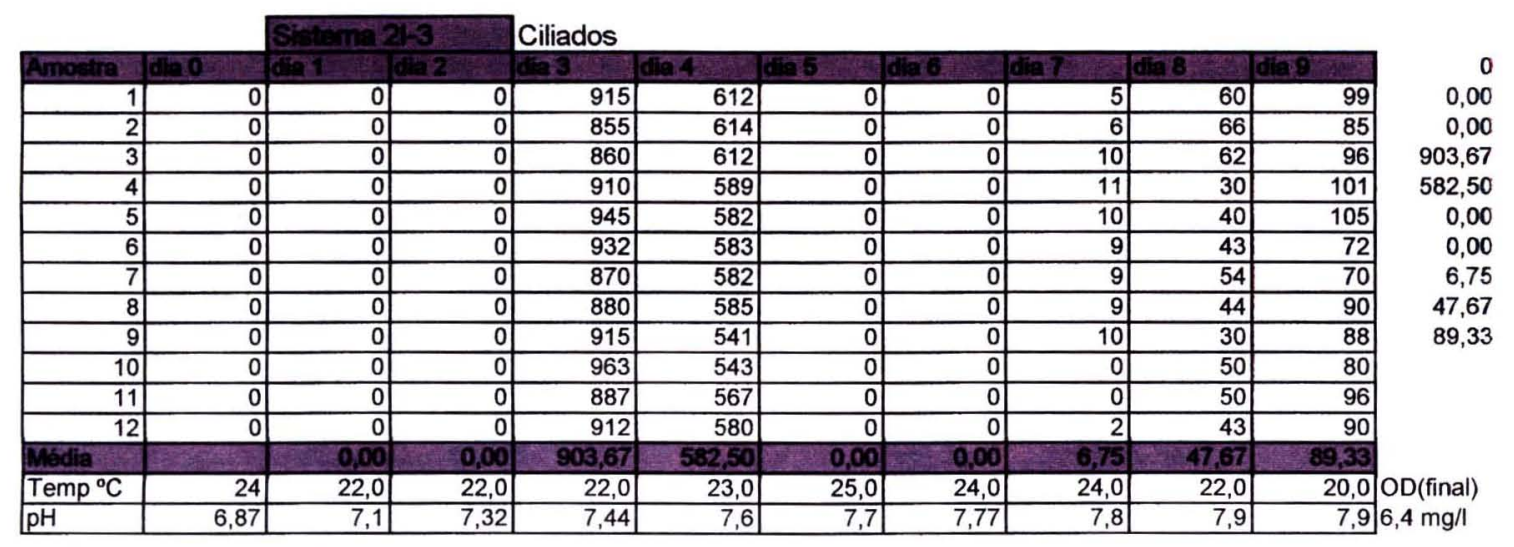

\begin{tabular}{|c|c|c|c|c|c|c|c|c|c|c|c|}
\hline & & Sistema: & $3 \vdash-3$ & Ciliados & & & & & & & \\
\hline Amostra & dia 0 & dia 1 & dia 2 & dia 3 & dia 4 & dia 5 & dia 6 & dia 7 & dia 8 & Tdia9 & c \\
\hline 1 & 1 & 0 & 0 & 565 & 715 & 0 & 0 & 0 & 0 & 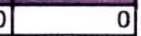 & 0,00 \\
\hline 2 & 0 & 0 & 0 & 542 & 690 & 0 & 0 & 0 & 0 & 0 & 0,00 \\
\hline 3 & 0 & 0 & 0 & 554 & 715 & 0 & 0 & 0 & 0 & 0 & 505,58 \\
\hline 4 & 0 & 0 & 0 & 490 & 705 & 0 & 0 & 0 & 0 & 0 & 702,83 \\
\hline 5 & 0 & 0 & 0 & 487 & 662 & 0 & 0 & 0 & 0 & 0 & 0,00 \\
\hline 6 & 0 & 0 & 0 & 455 & 672 & 0 & 0 & 0 & 0 & 0 & 0,00 \\
\hline 7 & 0 & 0 & 0 & 490 & 691 & 0 & 0 & 0 & 0 & 0 & 0,00 \\
\hline 8 & 0 & 0 & 0 & 487 & 711 & 0 & 0 & 0 & 0 & 0 & 0,00 \\
\hline 9 & 0 & 0 & 0 & 465 & 714 & 0 & 0 & 0 & 0 & 0 & 0,00 \\
\hline 10 & 0 & 0 & 0 & 512 & 702 & 0 & 0 & 0 & 0 & 0 & \\
\hline 11 & 0 & 0 & 0 & 524 & 736 & 0 & 0 & 0 & 0 & 0 & \\
\hline 12 & 0 & 0 & 0 & 496 & 721 & 0 & 0 & 0 & 0 & 0 & \\
\hline Madia & & 0,00 & 0,00 & 505,58 & 702,83 & 0,00 & 0,00 & 0,00 & 0,00 & 0,00 & \\
\hline Temp ${ }^{\circ} \mathrm{C}$ & 24 & 22,0 & 22,0 & 23,0 & 23,5 & 22,5 & 24,0 & 0,0 & 0,0 & 0,0 & $\mathrm{OD}$ (final) \\
\hline $\mathrm{pH}$ & 6,85 & 7 & 7,1 & 7,17 & 6,91 & 6,63 & 6,63 & 0 & 0 & 0 & $5,2 \mathrm{mg} / \mathrm{l}$ \\
\hline
\end{tabular}

\begin{tabular}{|c|c|c|c|c|c|c|c|c|c|c|c|}
\hline & & Sistema. & $4-3$ & Ciliados & & & & & & & \\
\hline Amostra & dia 0 & dia 1 & dia 2 & dia 3 & dia 4 & dias 5 & dia 6 & dia 7 & dia 8 & dia 9 & 0 \\
\hline 1 & 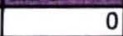 & 0 & 0 & 990 & 885 & 0 & 0 & 0 & 44 & 80 & 0,00 \\
\hline 2 & 0 & 0 & 0 & 985 & 902 & 0 & 0 & 0 & 42 & 82 & 0,00 \\
\hline 3 & 0 & 0 & 0 & 870 & 914 & 0 & 0 & 0 & 40 & 75 & 852,83 \\
\hline 4 & 0 & 0 & 0 & 885 & 817 & 0 & 0 & 0 & 30 & 77 & 940,75 \\
\hline 5 & 0 & 0 & 0 & 870 & 982 & 0 & 0 & 0 & 22 & 78 & 0,00 \\
\hline 6 & 0 & 0 & 0 & 854 & 963 & 0 & 0 & 0 & 20 & 92 & 0,00 \\
\hline 7 & 0 & 0 & 0 & 769 & 891 & 0 & 0 & 0 & 25 & 90 & 0,00 \\
\hline 8 & 0 & 0 & 0 & 763 & 889 & 0 & 0 & 0 & 30 & 95 & 30,00 \\
\hline 9 & 0 & 0 & 0 & 815 & 1009 & 0 & 0 & 0 & 19 & 80 & 83,08 \\
\hline 10 & 0 & 0 & 0 & 800 & 1112 & 0 & 0 & 0 & 29 & 73 & \\
\hline 11 & 0 & 0 & 0 & 810 & 1024 & 0 & 0 & 0 & 30 & 80 & \\
\hline 12 & 0 & 0 & 0 & 823 & 901 & 0 & 0 & 0 & 29 & 95 & \\
\hline Media & & 0,00 & 0,00 & 852,83 & 940,75 & 0,00 & 0,00 & 0,00 & 30,00 & 83.08 & \\
\hline Temp ${ }^{\circ} \mathrm{C}$ & 24 & 23,0 & 22,0 & 22,0 & 23,0 & 25,0 & 24,0 & 24,0 & 22,0 & 20,0 & $\mathrm{OD}$ (final) \\
\hline $\mathrm{pH}$ & 6,84 & 7,15 & 7,26 & 7,3 & 7,59 & 7,42 & 7,57 & 7,67 & 7,9 & 8 & $6,2 \mathrm{mg} / \mathrm{l}$ \\
\hline
\end{tabular}

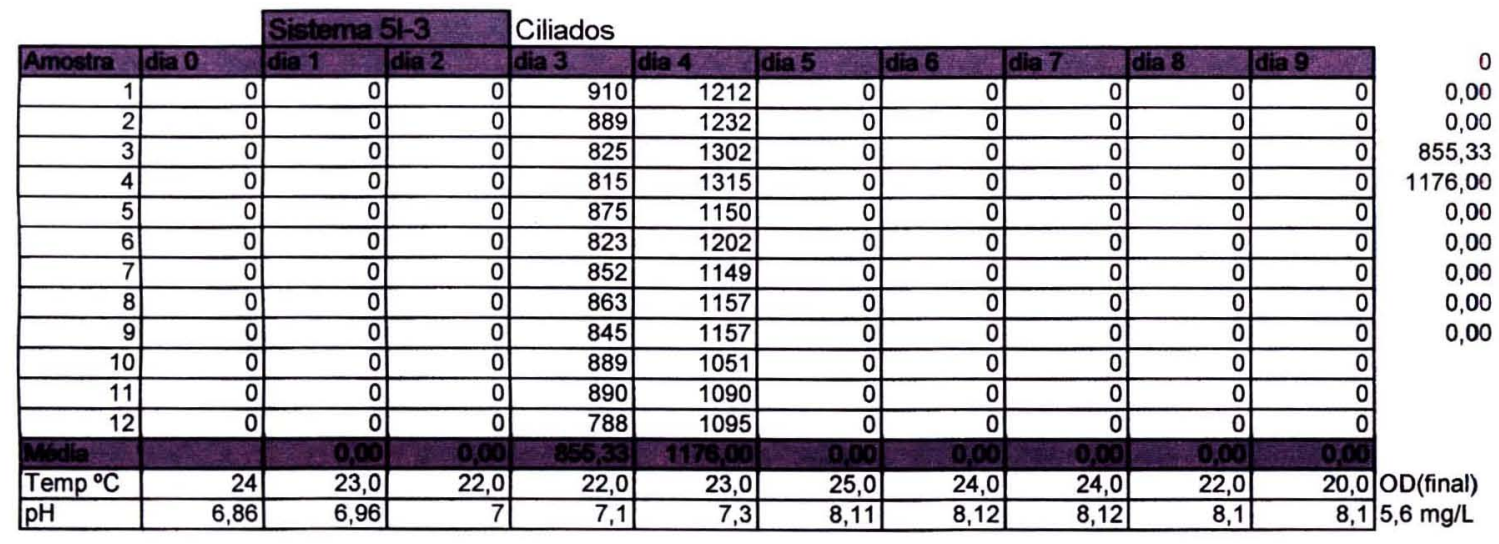




\section{ANEXO 2}

\section{KRUSKAL-WALLIS 1- ANOVA - ANÁ LISE DE VARIÂNCIA $(\alpha=0,05)$} EXPERIÊNCIA I - Sulfato de Cobre - Dinâ mica de crescimento de microrganismos.

Legenda:

$\mathrm{X}=$ Bactérias filamentosas

$X(1$ a 9$)=$ dia amostrado

$\mathrm{M}=$ Protozoários ciliados $M(1$ a 9$)=$ dia amostrado

$\mathrm{X} 1$

\begin{tabular}{cccc} 
Mean Rank & \multicolumn{3}{l}{ Cases } \\
18,00 & 5 & TIPOR $=1$ & C0 \\
5,50 & 5 & TIPOR $=2$ & C1 \\
5,50 & 5 & TIPOR $=3$ & C2 \\
13,00 & 5 & TIPOR $=4$ & C3
\end{tabular}

Chi-Square

D.F.

Significance

, 0011

Chi-Square

18,6681

D.F. Significance

3,0003

\begin{tabular}{llll} 
X2 \\
\multicolumn{1}{l}{ Mean Rank } & Cases \\
17,00 & 5 & TIPOR $=1$ & C0 \\
10,60 & 5 & TIPOR $=2$ & C1 \\
10,50 & 5 & TIPOR $=3$ & C2 \\
3,90 & 5 & TIPOR $=4$ & C 3
\end{tabular}

Chi-Square D.F.

12,2600

3

Significance

, 0065

Chi-Square

12,3342
D.F. Significance

$3 \quad, 0063$

X3

Mean Rank Cases

$\begin{array}{clll}18,00 & 5 & \text { TIPOR }=1 & \text { C0 } \\ 11,70 & 5 & \text { TIPOR }=2 & \text { C1 } \\ 9,30 & 5 & \text { TIPOR }=3 & \text { C2 } \\ 3,00 & 5 & \text { TIPOR }=4 & \text { C3 }\end{array}$

Chi-Square D.F.

Significance

, 0009

Chi-Square

16,5326

D.F. Significance

16,4829

3

,0009

$3 \quad, 0009$ 
$\mathrm{X} 4$

Mean Rank Cases

$\begin{array}{lll}7,50 & 5 & \text { TIPOR }\end{array}=1 \quad \mathrm{C} 0$

6,805 TIPOR $=2 \quad \mathrm{Cl}$

$11,50 \quad 5 \quad$ TIPOR $=3 \quad \mathrm{C} 2$

$16,20 \quad 5 \quad$ TIPOR $=4 \quad \mathrm{C} 3$

Chi-Square D.F. Significance

8,0257

3

, 0455

Chi-Square

8,1049
D.F. Significance

3

, 0439

\section{X5}

Mean Rank Cases

$13,00 \quad 5$ TIPOR $=1 \quad \mathrm{C} 0$

$15,60 \quad 5$ TIPOR $=2 \quad \mathrm{Cl}$

$7,70 \quad 5 \quad$ TIPOR $=3 \quad \mathrm{C} 2$

$5,70 \quad 5$ TIPOR $=4 \quad \mathrm{C} 3$

Chi-Square D.F. Significance

9,0200

3

, 0290

Chi-Square

9,0952

D.F. Significance

3

, 0281

X6

Mean Rank Cases

$14,40 \quad 5 \quad$ TIPOR $=1 \quad$ C0

$15,00 \quad 5$ TIPOR $=2 \quad \mathrm{Cl}$

$8,80 \quad 5 \quad$ TIPOR $=3 \quad \mathrm{C} 2$

$3,80 \quad 5 \quad \mathrm{TIPOR}=4 \quad \mathrm{C} 3$

Chi-Square D.F. Significance

$11,8914 \quad 3$

, 0078

Chi-Square

11,9004

D.F. Significance

$3 \quad 0077$

X7

Mean Rank Cases

$9,00 \quad 5 \quad$ TIPOR $=1 \quad$ C0

$14,60 \quad 5 \quad$ TIPOR $=2 \quad \mathrm{Cl}$

$13,90 \quad 5 \quad$ TIPOR $=3 \quad \mathrm{C} 2$

$4,50 \quad 5$ TIPOR $=4 \quad \mathrm{C} 3$

Chi-Square D.F.

$9,5171 \quad 3$

Significance

, 0231

Chi-Square

9,6038

D.F. Significance

3

, 0223 
X8

Mean Rank Cases

$\begin{array}{llll}13,10 & 5 & \text { TIPOR }=1 & \text { C0 } \\ 11,90 & 5 & \text { TIPOR }=2 & \text { C1 } \\ 11,80 & 5 & \text { TIPOR }=3 & \text { C2 } \\ 5,20 & 5 & \text { TIPOR }=4 & \text { C3 }\end{array}$

Chi-Square D.F. Significance , 1386

Chi-Square

5,6530

D.F. Significance

3

,1298

X9

Mean Rank Cases

$13,80 \quad 5 \quad$ TIPOR $=1 \quad$ C0

$8,20 \quad 5 \quad$ TIPOR $=2 \quad \mathrm{Cl}$

$12,10 \quad 5 \quad$ TIPOR $=3 \quad \mathrm{C} 2$

$7,90 \quad 5 \quad$ TIPOR $=4 \quad \mathrm{C} 3$

Chi-Square D.F. Significance

3,6429

3

, 3027

Chi-Square

3,6788

D.F. Significance

$3 \quad 2983$

M3

Mean Rank Cases

$12,50 \quad 5 \quad$ TIPOR $=1 \quad \mathrm{C} 0$

$8,20 \quad 5 \quad$ TIPOR $=2 \quad \mathrm{Cl}$

$9,90 \quad 5 \quad$ TIPOR $=3 \quad \mathrm{C} 2$

$11,40 \quad 5$ TIPOR $=4 \quad \mathrm{C} 3$

Chi-Square D.F. Significance

$1,4943 \quad 3$

, 6836

Chi-Square

1,5067

D.F. Significance

$3 \quad, 6807$

M4

Mean Rank Cases

$17,00 \quad 5 \quad$ TIPOR $=1 \quad \mathrm{C} 0$

8,005 TIPOR $=2 \quad \mathrm{Cl}$

$8,40 \quad 5 \quad$ TIPOR $=3 \quad$ C2

$8,60 \quad 5$ TIPOR $=4 \quad \mathrm{C} 3$

$\begin{array}{crr}\text { Chi-Square } & \text { D.F. } & \text { Significance } \\ 8,0743 & 3 & , 0445\end{array}$

$0445 \quad 8,0743$

D.F. Significance

$3 \quad, 0445$

M5

Mean Rank Cases

$15,60 \quad 5$ TIPOR $=1 \quad \mathrm{CO}$

$14,60 \quad 5 \quad$ TIPOR $=2 \quad \mathrm{Cl}$

$8,80 \quad 5 \quad$ TIPOR $=3 \quad \mathrm{C} 2$

$3,00 \quad 5$ TIPOR $=4 \quad \mathrm{C} 3$

Chi-Square D.F.

Significance

$14,5657 \quad 3$

, 0022

Chi-Square

D.F. Significance

14,6097

3

, 0022 
M6

Mean Rank Cases

$15,00 \quad 5 \quad$ TIPOR $=1 \quad$ C0

14,005 TIPOR $=2 \quad \mathrm{Cl}$

$10,00 \quad 5 \quad$ TIPOR $=3 \quad \mathrm{C} 2$

$3,00 \quad 5$ TIPOR $=4 \quad \mathrm{C} 3$

Chi-Square D.F

$12,7143 \quad 3$

Significance

, 0053
Chi-Square

12,9084
D.F. Significance

3 , 0048

M7

Mean Rank Cases

$14,00 \quad 5 \quad$ TIPOR $=1 \quad \mathrm{C} 0$

$12,40 \quad 5 \quad$ TIPOR $=2 \quad \mathrm{Cl}$

$12,60 \quad 5 \quad$ TIPOR $=3 \quad \mathrm{C} 2$

$3,00 \quad 5$ TIPOR $=4 \quad \mathrm{C} 3$

Chi-Square D.F.

$10,9314 \quad 3$

Significance

, 0121

Chi-Square

11,0142

D.F. Significance

$3 \quad, 0116$

\section{M8}

Mean Rank Cases

$11,40 \quad 5 \quad$ TIPOR $=1 \quad \mathrm{C} 0$

$13,80 \quad 5 \quad$ TIPOR $=2 \quad \mathrm{Cl}$

$13,80 \quad 5 \quad$ TIPOR $=3 \quad \mathrm{C} 2$

$3,00 \quad 5 \quad \mathrm{TIPOR}=4 \quad \mathrm{C} 3$

Chi-Square D.F.

$11,2629 \quad 3$

Significance

, 0104

Chi-Square

11,4348

D.F. Significance

$3 \quad, 0096$

M9

Mean Rank Cases

$15,60 \quad 5 \quad$ TIPOR $=1 \quad$ C 0

$13,40 \quad 5 \quad$ TIPOR $=2 \quad \mathrm{Cl}$

$9,50 \quad 5 \quad$ TIPOR $=3 \quad \mathrm{C} 2$

$3,50 \quad 5 \quad$ TIPOR $=4 \quad$ C3

Chi-Square D.F.

$12,0600 \quad 3$

Significance

, 0072

Chi-Square

12,3859
D.F. Significance

$3 \quad, 0062$ 
EXPERIÊNCIA II - Sulfato de Cobre - Dinâ mica de crescimento de microrganismos.

Legenda:

$\mathrm{X}=$ Bactérias filamentosas

$X(1$ a 9$)=$ dia amostrado

$\mathrm{M}=$ Protozoários ciliados

$M(1$ a 9$)=$ dia amostrado

$\mathrm{X} 1$

Mean Rank Cases

$12,00 \quad 5 \quad$ TIPOR $=1 \quad \mathrm{C} 0$

$14,40 \quad 5 \quad$ TIPOR $=2 \quad \mathrm{Cl}$

$11,80 \quad 5 \quad$ TIPOR $=3 \quad \mathrm{C} 2$

$3,80 \quad 5 \quad$ TIPOR $=4 \quad \mathrm{C} 3$

Chi-Square D.F

9,1486

3

Significance

, 0274

Chi-Square D.F. Significance

$\begin{array}{lll}9,5582 & 3 & , 0227\end{array}$

$\mathrm{X} 2$

Mean Rank Cases

$12,60 \quad 5 \quad$ TIPOR $=1 \quad \mathrm{C} 0$

$12,70 \quad 5 \quad$ TIPOR $=2 \quad \mathrm{Cl}$

$6,90 \quad 5 \quad$ TIPOR $=3 \quad \mathrm{C} 2$

$9,80 \quad 5 \quad$ TIPOR $=4 \quad \mathrm{C} 3$

Chi-Square D.F. Significance

3,2429

3

,3557

$\begin{array}{rr}\text { Chi-Square } & \text { D.F. } \\ 3,2453 & 3\end{array}$

Significance

, 3553

X3

Mean Rank Cases

$15,90 \quad 5 \quad$ TIPOR $=1 \quad \mathrm{C} 0$

$9,00 \quad 5 \quad$ TIPOR $=2 \quad \mathrm{Cl}$

$8,60 \quad 5 \quad$ TIPOR $=3 \quad \mathrm{C} 2$

$8,50 \quad 5 \quad$ TIPOR $=4 \quad \mathrm{C} 3$

Chi-Square

D.F.

3

Significance

, 1343

Chi-Square D.F.

$5,6038 \quad 3$

Significance

,1326

$\mathrm{X} 4$

Mean Rank Cases

$13,50 \quad 5 \quad$ TIPOR $=1 \quad \mathrm{C} 0$

$15,00 \quad 5$ TIPOR $=2 \quad \mathrm{Cl}$

$8,00 \quad 5 \quad$ TIPOR $=3 \quad$ C2

$5,50 \quad 5 \quad$ TIPOR $=4 \quad \mathrm{C} 3$

Chi-Square D.F.

8,6429

3

Significance

, 0344

Chi-Square D.F.

Significance

$9,9352 \quad 3$

, 0191 
X5

Mean Rank Cases

$14,20 \quad 5 \quad$ TIPOR $=1 \quad$ C0

$16,80 \quad 5 \quad$ TIPOR $=2 \quad \mathrm{Cl}$

$4,00 \quad 5 \quad$ TIPOR $=3 \quad \mathrm{C} 2$

$7,00 \quad 5$ TIPOR $=4 \quad \mathrm{C} 3$

Chi-Square D.F.

$15,4114 \quad 3$

$\mathrm{X} 6$

Mean Rank Cases

$15,90 \quad 5 \quad$ TIPOR $=1 \quad \mathrm{C} 0$

$14,10 \quad 5 \quad$ TIPOR $=2 \quad \mathrm{Cl}$

$7,50 \quad 5 \quad$ TIPOR $=3 \quad \mathrm{C} 2$

$4,50 \quad 5 \quad$ TIPOR $=4 \quad \mathrm{C} 3$

Chi-Square

12,4457

D.F.

3
Significance

, 0015
Chi-Square D.F. $16,1015 \quad 3$

Significance , 0011

X7

Mean Rank Cases

$\begin{array}{llll}12,20 & 5 & \text { TIPOR }=1 & \mathrm{C} 0 \\ 16,70 & 5 & \text { TIPOR }=2 & \mathrm{C} 1 \\ 7,80 & 5 & \text { TIPOR }=3 & \mathrm{C} 2 \\ 5,30 & 5 & \text { TIPOR }=4 & \mathrm{C} 3\end{array}$

Chi-Square D.F.

Significance

, 0128

Chi-Square

D.F. Significance

, 0060

$13,2954 \quad 3$

, 0040

Chi-Square D.F. Significance $10,9153 \quad 3 \quad 0122$

$\mathrm{X} 8$

Mean Rank Cases

$15,10 \quad 5 \quad$ TIPOR $=1 \quad \mathrm{C} 0$

$14,90 \quad 5$ TIPOR $=2 \quad \mathrm{Cl}$

$\begin{array}{lll}7,20 & 5 & \text { TIPOR }=3 \\ \mathrm{C} 2\end{array}$

$4,80 \quad 5 \quad$ TIPOR $=4 \quad \mathrm{C} 3$

Chi-Square D.F.

$11,9857 \quad 3$

Significance

, 0074

Chi-Square D.F. Significance $\begin{array}{lll}12,5224 \quad 3 & , 0058\end{array}$

X9

Mean Rank Cases

$16,60 \quad 5 \quad$ TIPOR $=1 \quad \mathrm{C} 0$

$11,80 \quad 5 \quad$ TIPOR $=2 \quad \mathrm{Cl}$

$10,10 \quad 5 \quad$ TIPOR $=3 \quad \mathrm{C} 2$

$3,50 \quad 5 \quad$ TIPOR $=4 \quad \mathrm{C} 3$

Chi-Square

D.F.

12,5800

3

Significance

,0056

Chi-Square D.F. Significance $12,9600 \quad 3 \quad, 0047$ 
M3

Mean Rank Cases

$12.50 \quad 5 \quad$ TIPOR $=1 \quad \mathrm{C} 0$

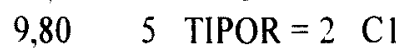

$9,80 \quad 5 \quad$ TIPOR $=3 \quad \mathrm{C} 2$

$9,90 \quad 5 \quad$ TIPOR $=4 \quad \mathrm{C} 3$

$\begin{array}{crrrrr}\text { Chi-Square } & \text { D.F. } & \text { Significance } & \text { Chi-Square } & \text { D.F. } & \text { Significance } \\ , 7629 & 3 & , 8583 & , 7841 & 3 & , 8533\end{array}$

M4

Mean Rank Cases

$11,60 \quad 5 \quad$ TIPOR $=1 \quad \mathrm{C} 0$

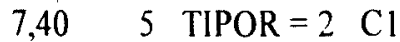

$12,60 \quad 5 \quad$ TIPOR $=3 \quad \mathrm{C} 2$

$10,40 \quad 5$ TIPOR $=4 \quad \mathrm{C} 3$

Chi-Square D.F.

$2,1771 \quad 3$

Significance

, 5365

Chi-Square D.F. Significance

$\begin{array}{lll}2,1771 & 3 & 5365\end{array}$

M5

Mean Rank Cases

$17,40 \quad 5 \quad$ TIPOR $=1 \quad \mathrm{C} 0$

$13,60 \quad 5 \quad$ TIPOR $=2 \quad \mathrm{Cl}$

$8,00 \quad 5 \quad$ TIPOR $=3 \quad \mathrm{C} 2$

$3,00 \quad 5 \quad$ TIPOR $=4 \quad \mathrm{C} 3$

Chi-Square D.F.

$17,1029 \quad 3$

Significance

, 0007

Chi-Square

D.F. Significance

$\begin{array}{lll}17,1029 & 3 & , 0007\end{array}$

M6

Mean Rank Cases

$18,00 \quad 5 \quad$ TIPOR $=1 \quad \mathrm{C} 0$

$12,60 \quad 5 \quad$ TIPOR $=2 \quad \mathrm{Cl}$

$8,40 \quad 5 \quad$ TIPOR $=3 \quad \mathrm{C} 2$

$3,00 \quad 5 \quad$ TIPOR $=4 \quad \mathrm{C} 3$

Chi-Square D.F.

$17,3314 \quad 3$

Significance

, 0006

Chi-Square D.F. Significance

$\begin{array}{lll}17,3314 \quad 3 & 0006\end{array}$

M7

Mean Rank Cases

$14,60 \quad 5 \quad$ TIPOR $=1 \quad \mathrm{C} 0$

$13,40 \quad 5 \quad$ TIPOR $=2 \quad \mathrm{Cl}$

$11,00 \quad 5 \quad$ TIPOR $=3 \quad \mathrm{C} 2$

$3,00 \quad 5 \quad$ TIPOR $=4 \quad \mathrm{C} 3$

Chi-Square D.F.

$11,6743 \quad 3$

Significance

, 0086

Chi-Square

11,7627
D.F. Significance

$3 \quad, 0082$ 
M8

Mean Rank Cases

$15,20 \quad 5 \quad$ TIPOR $=1 \quad \mathrm{C} 0$

$13,00 \quad 5 \quad$ TIPOR $=2 \quad \mathrm{Cl}$

$10,80 \quad 5 \quad$ TIPOR $=3 \quad \mathrm{C} 2$

$3,00 \quad 5 \quad$ TIPOR $=4 \quad \mathrm{C} 3$

Chi-Square D.F.

12,0971

3
Significance

, 0071
Chi-Square D.F. Significance

$12,2818 \quad 3$

M9

Mean Rank Cases
$15.60 \quad 5 \quad$ TIPOR $=1 \quad \mathrm{C} 0$
$13,60 \quad 5 \quad$ TIPOR $=2 \quad \mathrm{Cl}$
$8,80 \quad 5 \quad$ TIPOR $=3 \quad \mathrm{C} 2$
$4,00 \quad 5 \quad$ TIPOR $=4 \quad \mathrm{C} 3$

Chi-Square D.F

11,5371

3

Significance

Chi-Square

D.F.

Significance

$\begin{array}{lll}12,0443 \quad 3 & , 0072\end{array}$ 
EXPERIÊ NCIA III - Inseticida DDVP 20 - Dinâ mica de crescimento de microrganismos

Legenda:

$\mathrm{X}=$ Bactérias filamentosas

$X(1$ a 9$)=$ dia amostrado

$\mathrm{M}=$ Protozoários ciliados

$\mathrm{M}(1$ a 9$)=$ dia amostrado

$\mathrm{X} 1$

Mean Rank Cases

$12,80 \quad 5 \quad$ TIPOR $=5 \quad 10$

$11,70 \quad 5 \quad$ TIPOR $=6 \quad 11$

$11,10 \quad 5 \quad$ TIPOR $=7 \quad 12$

$6,40 \quad 5 \quad$ TIPOR $=8 \quad \mathrm{I} 3$

Chi-Square D.F.

3

Significance

, 3321

Chi-Square

D.F.

Significance

$\begin{array}{lll}3,4402 & 3286\end{array}$

$\mathrm{X} 2$

Mean Rank Cases

$16,00 \quad 5 \quad$ TIPOR $=5 \quad 10$

$11,90 \quad 5 \quad$ TIPOR $=6 \quad$ II

$\begin{array}{llll}6,50 & 5 & \text { TIPOR }=7 & \text { I2 }\end{array}$

$\begin{array}{lll}7,60 & 5 & \text { TIPOR }=8\end{array}$

Chi-Square D.F. Significance

8,0886

3

, 0442

Chi-Square

8,1191

D.F. Significance

$3 \quad, 0436$

$\mathrm{X} 3$

Mean Rank Cases

$14,70 \quad 5 \quad$ TIPOR $=5 \quad 10$

$12,30 \quad 5 \quad$ TIPOR $=6 \quad$ II

$\begin{array}{llll}7,80 & 5 & \text { TIPOR }=7 & 12\end{array}$

$7,20 \quad 5 \quad$ TIPOR $=8 \quad 13$

Chi-Square D.F.

5,5800

3

Significance

, 1339

Chi-Square

D.F.

Significance

5,5842

, 1337

$\mathrm{X} 4$

Mean Rank Cases

$\begin{array}{clll}14,20 & 5 & \text { TIPOR }=5 & 10 \\ 10,20 & 5 & \text { TIPOR }=6 & \text { I1 } \\ 8,70 & 5 & \text { TIPOR =7 } & 12 \\ 8,90 & 5 & \text { TIPOR }=8 & \text { I3 }\end{array}$

Chi-Square

D.F.

2,7971

3

Significance

,4240

Chi-Square

D.F.

Significance

2,8056

3

,4226 
X5

Mean Rank Cases

$\begin{array}{cccc}9,80 & 5 & \text { TIPOR }=5 & 10 \\ 13,00 & 5 & \text { TIPOR }=6 & 11 \\ 16,20 & 5 & \text { TIPOR }=7 & 12 \\ 3,00 & 5 & \text { TIPOR }=8 & 13\end{array}$

Chi-Square D.F.

13,6400
Significance

, 0034
Chi-Square

13,7538
D.F. 3

Significance , 0033

X6

Mean Rank Cases

$\begin{array}{clll}11,30 & 5 & \text { TIPOR }=5 & 10 \\ 14,50 & 5 & \text { TIPOR }=6 & I 1 \\ 13,00 & 5 & \text { TIPOR }=7 & 12 \\ 3,20 & 5 & \text { TIPOR }=8 & 13\end{array}$

Chi-Square

D.F.

10,8829

Significance

, 0124
D.F.

$10,9736 \quad 3$

Significance , 0119

X7

Mean Rank Cases

$\begin{array}{clll}11,40 & 5 & \text { TIPOR }=5 & 10 \\ 14,90 & 5 & \text { TIPOR }=6 & 11 \\ 11,90 & 5 & \text { TIPOR }=7 & 12 \\ 3,80 & 5 & \text { TIPOR }=8 & 13\end{array}$

Chi-Square D.F.

$9,5743 \quad 3$

Significance

, 0226

Chi-Square D.F.

$9,6688 \quad 3$

Significance

, 0216

$\mathrm{X} 8$

Mean Rank Cases

$12,60 \quad 5 \quad$ TIPOR $=5 \quad 10$

$14,30 \quad 5 \quad$ TIPOR $=6 \quad 11$

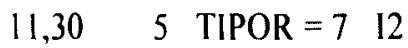

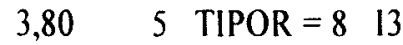

Chi-Square D.F.

$9,1971 \quad 3$

Significance

, 0268

Chi-Square D.F.

Significance

$9,2388 \quad 3$

, 0263

X9

Mean Rank Cases

$\begin{array}{lll}9,90 \quad 5 & \text { TIPOR }=5 & \text { I0 }\end{array}$

$12,60 \quad 5 \quad$ TIPOR $=6 \quad \mathrm{Il}$

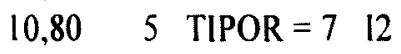

$8,70 \quad 5 \quad$ TIPOR $=8 \quad 13$

Chi-Square

D.F.

Significance

, 7633

Chi-Square D.F.

Significance

1,1571

3

$1,1624 \quad 3$

,7620 
M3

Mean Rank Cases

$9,00 \quad 5 \quad$ TIPOR $=5 \quad 10$

$9.60 \quad 5 \quad$ TIPOR $=6 \quad$ II

$9.80 \quad 5 \quad$ TIPOR $=7 \quad 12$

$13,60 \quad 5 \quad$ TIPOR $=8 \quad 13$

Chi-Square D.F.

1,8800
Significance

.5977
Chi-Square D.F. $1,8800 \quad 3$

Significance .5977

\section{M4}

Mean Rank Cases

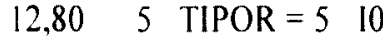

$9,40 \quad 5 \quad$ TIPOR $=6 \quad$ II

$\begin{array}{llll}12,00 & 5 & \text { TIPOR }=7 & 12\end{array}$

$\begin{array}{lll}7,80 & 5 & \text { TIPOR }=8\end{array}$

Chi-Square D.F.

2,2914

3

Significance

, 5142

Chi-Square D.F.

Significance

$2,2914 \quad 3$

, 5142

M5

Mean Rank Cases

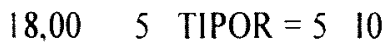

$12,40 \quad 5 \quad$ TIPOR $=6 \quad 11$

$\begin{array}{llll}8,60 & 5 & \text { TIPOR }=7 & 12\end{array}$

$3,00 \quad 5 \quad$ TIPOR $=8 \quad 13$

Chi-Square D.F.

$17,1029 \quad 3$

Significance

, 0007

Chi-Square D.F.

$17,3640 \quad 3$

Significance

, 0006

\section{M6}

Mean Rank Cases

$\begin{array}{llll}18,00 & 5 & \text { TIPOR }=5 & 10\end{array}$

$\begin{array}{lll}11.80 & 5 & \text { TIPOR }=6 \quad 11\end{array}$

$9,20 \quad 5 \quad$ TIPOR $=7 \quad 12$

$3,00 \quad 5 \quad$ TIPOR $=8 \quad 13$

$\begin{array}{cr}\text { Chi-Square } & \text { D.F. } \\ 16,5543 & 3\end{array}$

Significance

, 0009

Chi-Square

D.F. Significance

$\begin{array}{lll}16,8070 \quad 3 & , 0008\end{array}$

M7

Mean Rank Cases

$18,00 \quad 5 \quad$ TIPOR $=5 \quad 10$

$9,80 \quad 5 \quad$ TIPOR $=6 \quad$ II

$\begin{array}{lll}11,20 \quad 5 & \text { TIPOR }=7 \quad 12\end{array}$

$3,00 \quad 5 \quad$ TIPOR $=8 \quad 13$

Chi-Square

D.F.

16,2114

3

Significance

, 0010
Chi-Square

16,2603
D.F. Significance $3 \quad, 0010$ 
M8

Mean Rank Cases

$11,80 \quad 5 \quad$ TIPOR $=5 \quad 10$

$9,00 \quad 5 \quad$ TIPOR $=6 \quad$ II

$16,40 \quad 5 \quad$ TIPOR $=7 \quad 12$

$4,80 \quad 5 \quad$ TIPOR $=8 \quad 13$

Chi-Square D.F.

$10,1771 \quad 3$

M9

Mean Rank Cases

$\begin{array}{llll}8,60 & 5 & \text { TIPOR }=5 & 10\end{array}$

$8,40 \quad 5 \quad$ TIPOR $=6 \quad$ II

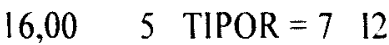

$9,00 \quad 5 \quad$ TIPOR $=8 \quad 13$

Chi-Square

D.F.

5,7886

3
Significance

, 0171

Significance

, 1224
Chi-Square

10.1848

D.F

3

Significance

, 0171
Chi-Squar
D.F.
Significance
$5.7929 \quad 3$
, 1221 